\title{
PHAGOCYTOSIS OF ANTIGENS BY LANGERHANS CELLS
}

Caetano Maria Pacheco Pais dos Reis e Sousa

A Thesis Presented for the Degree

of

Doctor of Philosophy

in the Faculty of Clinical Medicine,

University of Oxford

Michaelmas Term 1992

Nuffield Department of Surgery, John Radcliffe Hospital

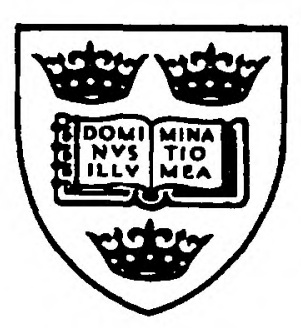

OIENOF

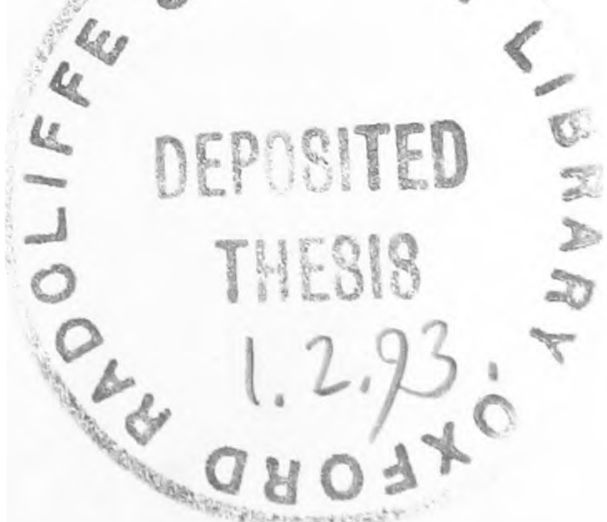

Hertford College,

Oxford 


\section{DEDICATION}

TO MY PARENTS,

Whose vision, love and example during the last twenty four years have inspired me to pursue intellectual achievement. 


\title{
PHAGOCYTOSIS OF ANTIGENS BY LANGERHANS CELLS
}

\author{
CAETANO REIS E SOUSA \\ D.PHIL. THESIS \\ HERTFORD COLLEGE \\ MICHAELMAS TERM 1992
}

\section{ABSTRACT}

Mature dendritic cells (DC) isolated from lymphoid tissues initiate antigen-specific Tdependent responses even though they are non-phagocytic and weakly pinocytic, whereas Langerhans cells (LC; immature DC) can process protein antigens but are poorly immunostimulatory. Thus antigens may be acquired by cells of this lineage at an immature stage but, to our knowledge, there have been no studies on the phagocytic capacity of these cells in vitro.

Using a newly-developed flow cytometric assay to measure the association between fluorescent markers and LC in epidermal cell cultures, and light and electron microscopy, we have observed phagocytosis of a variety of particles by freshly-isolated LC. The cells readily phagocytosed zymosan, heat-killed $S$. cerevisiae, bacteria ( $S$. aureus and $C$. parvum) and fluorescent latex beads, but were unable to take up IgG- or complementcoated sheep erythrocytes, as opposed to M $\varnothing$. Similarly, many freshly-isolated splenic DC had some phagocytic activity. However, the capacity of both LC and splenic DC to phagocytose zymosan, bacteria and fluorescent latex beads was markedly decreased after maturation in culture, consistently with the fact that mature DC are poorly phagocytic.

Zymosan binding and uptake were much greater in fresh LC from C57BL/6 compared to $\mathrm{BALB} / \mathrm{c}$ mice, and the loss of phagocytic capacity for zymosan during maturation followed different kinetics in the two strains. Two receptors mediating uptake of zymosan in LC were identified based on the effect of different inhibitors. Both of these receptors, recognising mannose and $\beta$-glucan residues, appear to be differentially regulated in the two mouse strains and during culture of LC.

Our findings support the notion that DC are capable of acquiring particulate antigens for presentation at an immature stage, through recognition units for carbohydrate determinants common to a variety of potentially pathogenic organisms. 


\section{ACKNOWLEDGEMENTS}

I would like to thank my supervisor, Dr. Jonathan Austyn, for his advice, support and friendship over the last three years and for initially showing me the heavenly light of dendrocytology while carefully concealing the infernal glow of DC purification. I thank Prof. P. J. Morris for giving me the opportunity to work in the Nuffield Department of Surgery and for financial support for some of the meetings I attended. I am extremely grateful to Prof. P. D. Stahl of the Department of Cell Biology and Physiology, Washington University School of Medicine, St. Louis, Missouri, USA, for inviting me to spend time in his laboratory and for provision of reagents, namely the antiserum against murine mannose receptor used in the experiments described in chapter 5. My thanks also to Prof. S. Gordon, Sir William Dunn School of Pathology, Oxford and to Dr. N. Romani, University of Innsbruck, Austria, for generous gifts of reagents and advice.

I am extremely indebted to Miss Zahida Ahmad for technical assistance and for keeping her good humour (and mine) when peeling countless mouse ears. Many of the experiments described in this thesis would never have been possible without her assistance because of the workload involved. I have benefited greatly from working alongside S. Ewing, A.S. Rao and J. Roake and I thank them for stimulating discussions, support and friendship and for making the Austyn lab such a pleasant environment. I would also like to acknowledge the many other people who offered advice and friendship during this course of this study: members of the Stahl lab, Romani lab and Gordon lab deserve special thanks for their time and reagents.

My research was made possible by a doctoral grant from the Program CIENCIA of the Portuguese scientific research council (JNICT) and a FCO award of The British Council, Lisbon, Portugal, to cover tuition and college fees at Oxford.

This thesis would never have been possible without the love and support of my family. I thank my parents and my brother for always being there and for encouraging my efforts. My parents gave me the opportunity to come to Oxford before I obtained a grant (and have given me every opportunity throughout my life...).

Last but not least, I am especially grateful to the Babycakes for preserving my sanity (or whatever remained of it) during this time, and for showing me the light at the end of the tunnel ... with a $2.5 \mathrm{ct}$. sparkle! 
"They was so little", he said, apologetically. "I'd pet 'em, and pretty soon they bit my fingers and I pinched their heads a little and then they was dead - because they was so little."

Of Mice And Men, John Steinbeck (1937). 


\section{ABBREVIATIONS}

ATCC

$\alpha-10$-thio

BCIP

BG

BSA

CD

CDNA

CR

CTL

DC

DNP

EC

EDTA

FACS

Fc $\gamma R$

FcR

FCS

FITC

GM-CSF

HBSS

HI

HPLC

Ia

IDC

IFN- $\gamma$

Ig

Ii

IL

$\mathrm{KCM}$

$\mathrm{KLH}$

LC

$\mathrm{LN}$

ManBSA

$\mathrm{MHC}$

MLR
American Tissue Culture Collection

Complete medium for culture of J774E cells

5-bromo-4-chloro-3-indolyl-phosphate

Birbeck granule

Bovine serum albumin

Cluster of differentiation (e.g. CD45)

complementary DNA

Complement receptor (e.g. CR3, CR type three)

Cytotoxic T lymphocyte

Dendritic cell(s)

Dinitrophenol

Epidermal cell(s)

Ethylenediaminetetraacetic acid

Fluorescence-activated cell sorter

Fc receptor for IgG

Fc receptor

Foetal calf serum

Fluorescein isothiocyanate

Granulocyte-macrophage colony-stimulating factor

Hank's balanced salt solution

Heat inactivated

High-performance liquid chromatography

Immune response-associated (MHC class II) antigens

Interdigitating cell

gamma-interferon

Immunoglobulin

Invariant chain

Interleukin (e.g. IL-1)

Keratinocyte-conditioned medium

Keyhole limpet haemocyanin

Langerhans cell(s)

Lymph node(s)

Mannosylated bovine serum albumin

Major histocompatibility complex

Mixed leukocyte reaction 


\begin{tabular}{ll} 
MØ & Macrophage(s) \\
MR & Mannose receptor \\
MW & Molecular weight \\
NBT & Nitroblue tetrazolium \\
NMS & Normal mouse serum \\
NRbS & Normal rabbit serum \\
NSE & Non-specific esterase \\
PAGE & Polyacrylamide gel electrophoresis \\
PBS & Cation-free phosphate buffered saline \\
PE & Phycoerythrin \\
PLL & Poly-L-lysine \\
PMSF & Phenylmethylsulfonyl fluoride \\
R0 & RPMI-1640 \\
R10 & Complete medium for primary cell cultures \\
Rb $\alpha M s ~ M R$ & Anti-mouse mannose receptor antibody \\
RBC & Red blood cells \\
SDS & Sodium dodecyl sulphate \\
SIg & surface immunoglobulin \\
SRBC & Sheep red blood cells \\
Thio-M & Thioglycollate-elicited macrophages \\
TNF & Tumour necrosis factor \\
TR & Texas Red \\
UV & Ultraviolet light \\
VC & Veiled cell \\
WS & Washing solution for immunofluorescence \\
& \\
\hline &
\end{tabular}




\section{Chapter 1. Introduction}

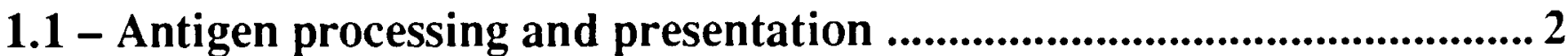

1.1.1 - Presentation of antigens by MHC class I molecules .................. 3

1.1.2 - Presentation of antigens by MHC class II molecules ................ 4

Biosynthesis and transport of MHC class II molecules ............. 5

Antigen capture and processing ............................................. 6

Association of MHC class II heterodimers and peptides ........... 7

1.2 - Dendritic cells .................................................................................................. 8

1.2.1 - Tissue distribution ................................................................. 10

Lymphoid dendritic cells ................................................... 11

Non-lymphoid dendritic cells .............................................. 12

Dendritic cells in circulation ................................................. 13

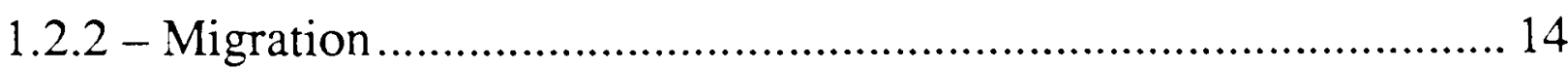

Migration via the lymph ........................................................ 14

Migration via the blood ...................................................... 15

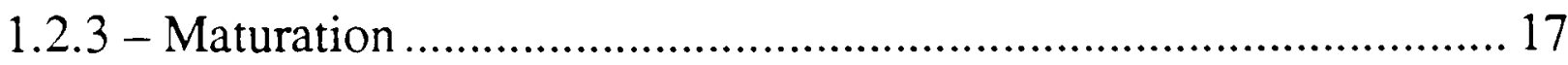

Phenotypic maturation ......................................................... 17

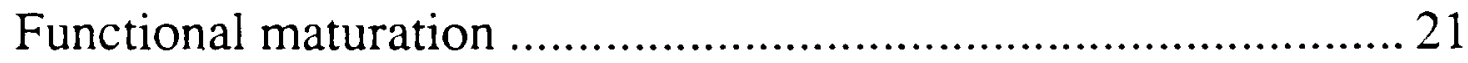

Regulation of LC maturation ............................................. 23

Maturation as a feature of DC ............................................. 23

1.2 .4 - T cell activation .................................................................... 26

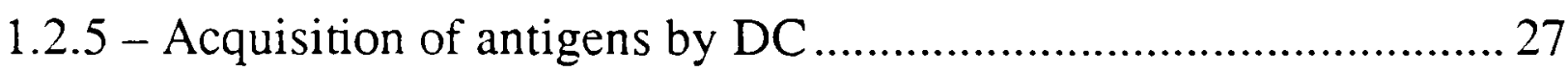

Antigen acquisition by splenic DC ......................................... 28

Immature DC in the periphery as sentinels of the immune

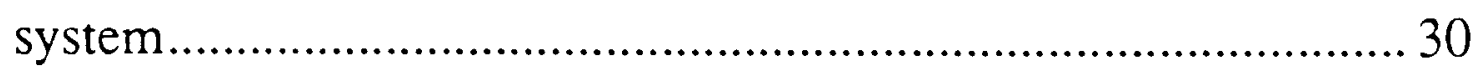

Phagocytosis of antigens by DC .......................................... 32

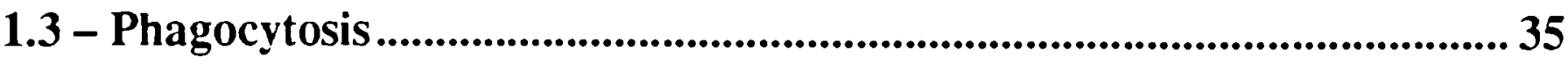

1.3.1 - The phagocytic pathway ....................................................... 35

1.3.2 - Receptors involved in phagocytosis ........................................ 39

Fcy receptors ............................................................... 40

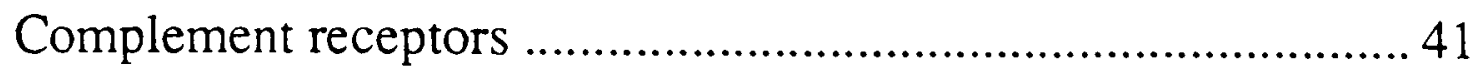

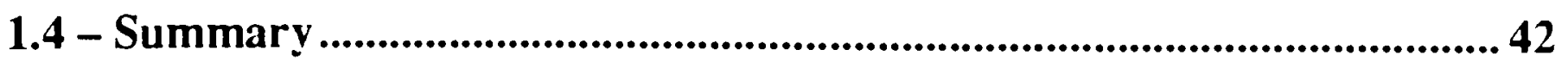

\section{Chapter 2. Materials and Methods}

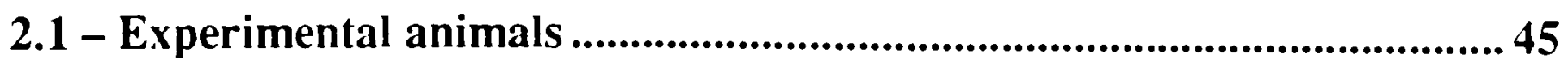

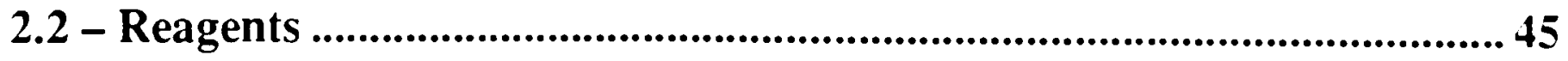


2.2.1 - Monoclonal antibodies ......................................................... 45

2.2.2 - Polyclonal antibodies ........................................................ 46

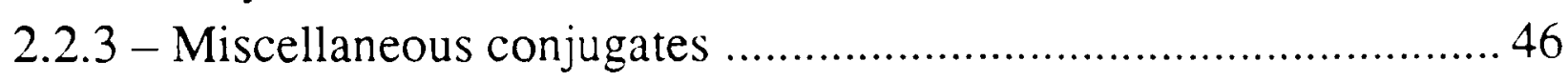

2.2.4 - Phagocytic markers ........................................................... 46

2.2 .5 - Endocytic markers and inhibitors .................................... 47

2.3 - Cell preparations .......................................................................................... 48

2.3.1 - Tissue culture reagents .................................................... 48

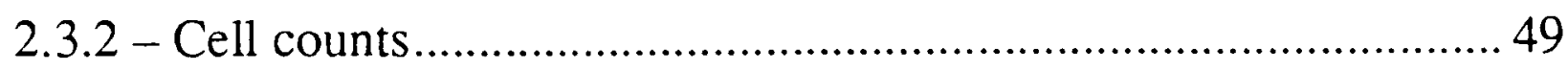

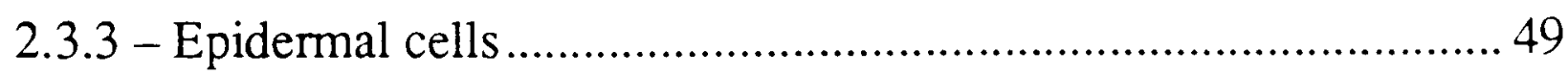

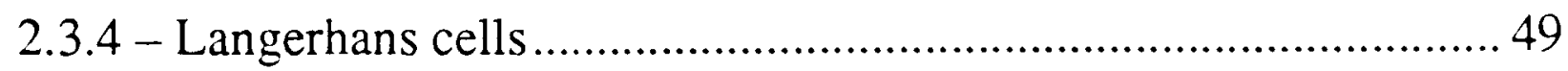

2.3.5 - Migratory Langerhans cells ............................................. 50

2.3.6 - Splenic dendritic cells ...................................................... 50

2.3.7 - Peritoneal macrophages .................................................. 51

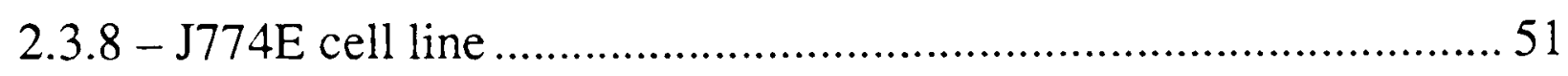

2.4 - Immunofluorescent cell labelling .......................................................... 52

2.4.3 - Attachment of cells to PLL-coverslips ................................... 52

2.4.4 - Immunofluorescence labelling of attached cells ...................... 52

2.4.5 - Immunofluorescence labelling for FACS ............................... 53

2.5 - Phagocytic and endocytic flow cytometric assays ................................. 54

2.5.1 - Phagocytic assays on Langerhans cells .................................. 54

2.5.2 - Differentiating between bound and internalised particles.......... 60

2.5.3 - Modification of the assay for splenic DC ................................62 62

2.5.4 - Modification of the assay for soluble tracers. .......................... 63

2.6 - Electron microscopy .........................................................................6 63

2.6.1 - Transmission electron microscopy (Langerhans cells) .............. 63

2.6.2 - Immuno-electron microscopy (splenic dendritic cells) ............ 64

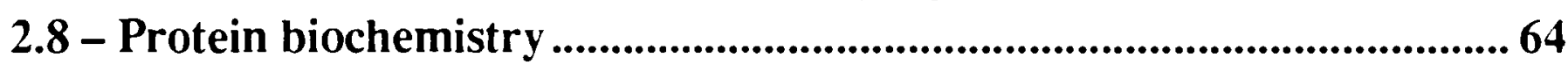

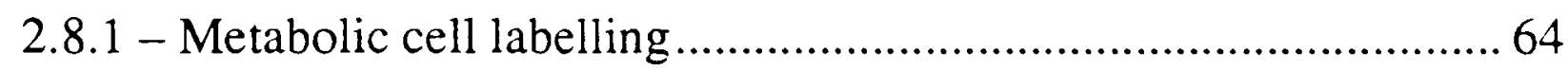

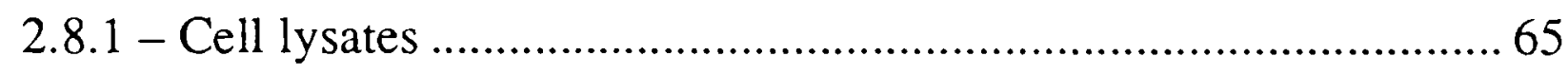

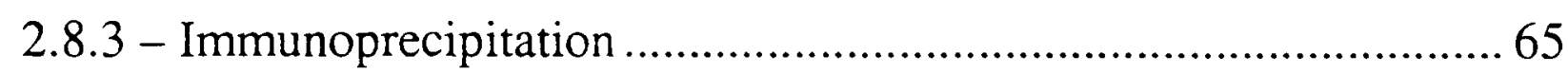

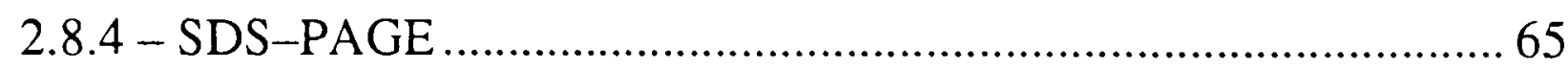

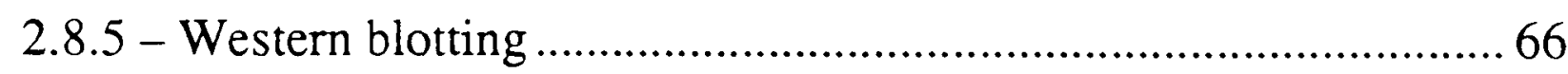

2.8.6 - Preparation of a $F\left(a b^{\prime}\right)_{2}$ fragment from anti-mouse mannose

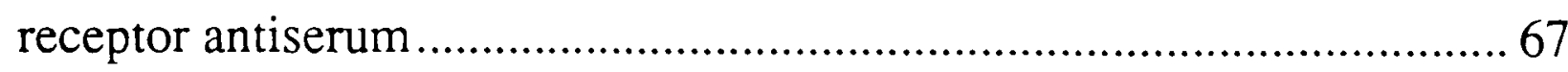

\section{Chapter 3. Freshly isolated murine Langerhans cells are phagocytic in} vitro

3.1 - Introduction 69

3.2 - Freshly isolated Langerhans cells are phagocytic in vitro 72

3.3 - Flow cytometric analysis of association between fluorescent particles and LC 
3.4 - Strain differences in the extent of zymosan uptake 89

3.5 - Comparison of Langerhans cells and macrophages .......................... 91

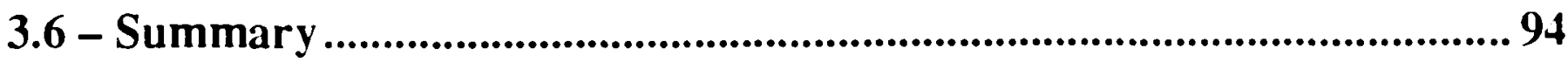

Chapter 4. Cultured Langerhans cells and splenic dendritic cells have little phagocytic capacity

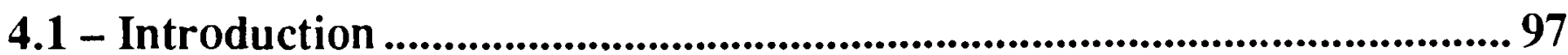

4.2 - Cultured LC have little phagocytic capacity .......................................... 99

4.3 - Pulsed LC retain and degrade particulate antigen during culture. .... 105

4.4 - Strain differences in the kinetics of down regulation of zymosan uptake

4.5 - Splenic DC resemble cultured LC in their phagocytic ability. ............ 110

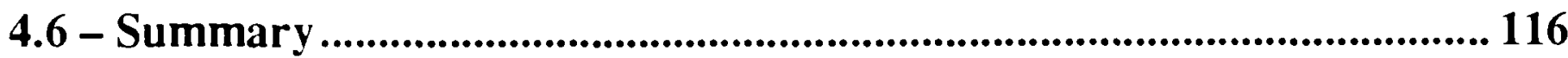

Chapter 5. Receptors involved in phagocytosis of zymosan by Langerhans cells

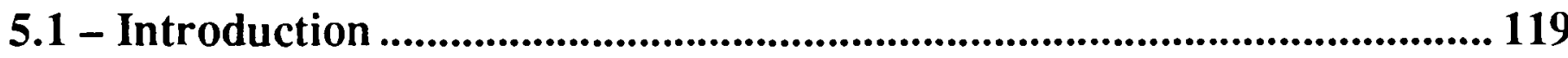

5.2 - Inhibition of zymosan uptake by LC ................................................ 124

5.2 .1 - Inhibition by soluble glycans .......................................... 124

5.2.2: Inhibition by anti-CR3 ................................................... 131

5.3 - Probing for mannose receptor on LC using soluble ligands ............... 135

5.3.1 - Studies on the uptake of mannosylated BSA ........................... 137

5.3.2 - Failure to find a fluorescent ligand to measure binding to $\mathrm{MR}$ rather than uptake ........................................................................ 143

5.4 - Biochemical analysis of mannose receptor expression by $L C$ and

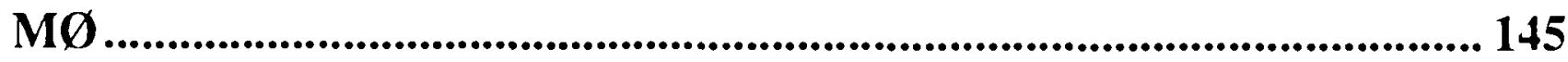

5.4.1 - Characterisation of a rabbit antiserum against murine macrophage mannose receptor

5.4.2 - Anti-mouse MR antiserum does not inhibit receptormediated uptake of MR ligands and is a poor reagent for immunofluorescence

5.4.3 - Analysis of MR expression in LC by Western blotting ............. 152

5.5 - Summary 157

\section{Chapter 6. Summary and Discussion}

6.1 - Summary

6.2 - Technical considerations 160

6.3 - Phagocytosis of antigens by dendritic cells 161 
6.4 - Receptors mediating phagocytosis by Langerhans cells..................... 166

6.5 - The relevance of phagocytosis by Langerhans cells............................. 171

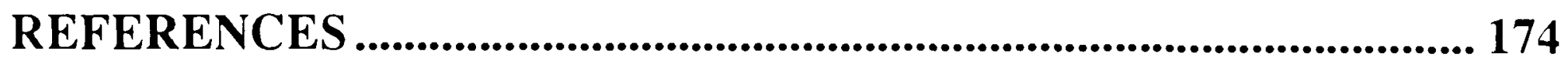




\section{Chapter 1}

\section{Introduction}

\section{1 - Antigen processing and presentation}

1.1.1 - Presentation of antigens by MHC class I molecules

1.1.2 - Presentation of antigens by MHC class II molecules

Biosynthesis and transport of MHC class II molecules

Antigen capture and processing

Association of MHC class II heterodimers and peptides

\section{2 - Dendritic cells}

1.2.1 - Tissue distribution

Lymphoid dendritic cells

Non-lymphoid dendritic cells

Dendritic cells in circulation

1.2.2 - Migration

Migration via the lymph

Migration via the blood

1.2.3 - Maturation

Phenotypic maturation

Functional maturation

Regulation of LC maturation

Maturation as a feature of DC

1.2.4 - $\mathrm{T}$ cell activation

1.2.5 - Acquisition of antigens by DC

Antigen acquisition by splenic DC

Immature DC in the periphery as sentinels of the immune

system

Phagocytosis of antigens by DC

\section{3 - Phagocytosis}

1.3.1 - The phagocytic pathway

1.3.2 - Receptors involved in phagocytosis

Fc $\gamma$ receptors

Complement receptors

\section{4 - Summary}




\section{1 - Antigen processing and presentation}

Immune responses to foreign antigens fall into two functional categories. Innate immunity encompasses a range of inherent defence mechanisms such as the anti-bacterial function of lysozyme in bodily secretions and the complement system of serum proteins. In contrast, acquired immunity is antigen-specific and is characterised by "memory", i.e., the capacity to mount an enhanced and more rapid immune response upon subsequent encounter with the same antigen. Central to the mechanism of acquired immunity is the participation of $T$ cells, particularly of the $T$ helper type. Unlike $B$ cells, $T$ cells do not recognise antigen in its native form. Instead, $\mathrm{T}$ cell receptors have evolved to recognise peptide fragments in association with major histocompatibility complex (MHC) molecules on cell surfaces. MHC molecules of the class I type, present on all nucleated cells, normally associate with peptides derived from endogenous antigens, such as cytosolic proteins, and are ideally suited to present viral antigens on infected cells [1]. MHC class II molecules (Ia) are primarily involved in presenting peptides derived from exogenous antigens (i.e., present in the external milieu) and are restricted to a more limited number of cell types, comprising dendritic cells, B cells and activated macrophages (MØ) [2]. Two mutually exclusive accessory molecules are expressed by distinct subsets of mature $T$ cells and determine their $\mathrm{MHC}$ restriction profile. CD4 molecules restrict T cells to MHC class II antigens whilst CD8 expression determines antigen recognition in the context of class I MHC molecules. It is generally the case that $\mathrm{CD} 8^{+} \mathrm{T}$ cells are involved in cytotoxic responses (cytotoxic $\mathrm{T}$ cells) whereas $\mathrm{CD} 4^{+} \mathrm{T}$ cells are concerned with cytokine secretion and regulation of the immune response (helper $\mathrm{T}$ cells). Any cell that presents peptide fragments on MHC molecules is, by definition, an antigen-presenting cell (APC). Thus, like Austyn [3], we use the term antigen-presenting cell to refer to any cell that expresses class II and/or class I MHC molecules on its surface and reserve the term accessory cell for the restricted group of APC that present antigen to $\mathrm{CD}^{+} \mathrm{T}$ cells. From an evolutionary point of view, it seems reasonable that every cell should have the ability to act as an APC 
for a cytotoxic $\mathrm{T}$ cell since any cell can potentially become virally infected and need to be destroyed. Accessory cells for class II-restricted responses often perform additional functions to antigen presentation such as activation of resting $\mathrm{T}$ cells (dendritic cells), elimination of antigen (MØ) or antibody secretion (B cells). The class I and class II pathways to antigen presentation are probably evolutionarily related but show important differences that are reviewed below. Detailed reviews can be found in references [1, 2, 4$6]$.

\subsection{1 - Presentation of antigens by MHC class I molecules}

MHC class I molecules are heterodimers of $\beta_{2}-$ microglobulin $(12 \mathrm{kD})$ and an $\alpha-$ chain of $45 \mathrm{kD}$ which contains a peptide-binding groove formed by interaction between the $\alpha 1$ and $\alpha 2$ domains [7]. Experiments where class I presentation was inhibited by the drug brefeldin A (BFA) first suggested that peptide-class I complexes might assemble in the endoplasmic reticulum (ER) [8,9]. However, interpretation of those experiments was complicated by the fact that class I molecules themselves have to exit the ER such that the effect of BFA was not specific for peptide loading alone. Much insight into the mechanisms of class I assembly has been gained from the study of mutant cell lines which cannot present endogenous antigens. One of these, the RMA-S mouse cell line has reduced levels of surface class I expression and the molecules do not contain peptide and are unstable at $37^{\circ} \mathrm{C}[10]$. Increased surface expression can be achieved by incubating the cells at $26^{\circ} \mathrm{C}$ or by feeding exogenous peptides [10]. It is believed that peptide or lowered temperature increase the stability of assembling complexes in the endoplasmic reticulum (ER) and allow these to travel to the cell surface. Thus, peptide plays an integral role in the assembly of class I molecules in the ER and helps achieve a transport-competent configuration. The defect of the RMA-S cell line is thought to be at the level of the peptide transport system that imports into the ER peptides generated by proteasomes and/or uther mechanisms in the cytosol $[1,6]$. Two genes, Tap-1 and Tap-2, each encode a subunit of a putative peptide transporter complex and transfection of Tap-2 cDNA into RMA-S cells 
restores the wild type phenotype [11]. Both the Tap genes and the Lmp genes which are thought to encode components of the putative cytosolic proteasomes are closely linked within the class II region of the MHC [6]. Polymorphisms in these genes could potentially dictate which peptides are presented by a given class I molecule and may have important implications for antigen presentation. Evidence suggests that this is indeed the case in the rat where a locus affecting class I determinants was recently mapped to the Tap-2 gene [12]. In addition, transporters may, conceivably, select peptides more suitable for binding to class I than those which are produced in the ER, possibly explaining why class I molecules in RMA-S cells do not assemble into a transport-competent conformation in the ER in spite of the abundance of peptides generated in that compartment [13].

Once assembled class I-peptide complexes travel through the Golgi where their oligosaccharide moieties are processed [14] and reach the cell surface. Some unassembled class I molecules may also reach the cell surface but the majority are retained in the ER and are degraded, possibly through the ER degradative pathway [13]. Peptide binding to MHC class I molecules is essentially irreversible [10] and assembled complexes dissociate rapidly at the cell surface if peptide is lost from the groove [15].

\subsection{2 - Presentation of antigens by MHC class II molecules}

Like class I, MHC class II component chains also assemble in the ER following biosynthesis but do not bind peptides in that compartment. Instead, class II molecules are thought to be transported to a compartment of the endocytic pathway where they meet incoming exogenous antigen. Three factors may, therefore, influence antigen presentation by class II molecules: 1) biosynthesis and transport of class II to the compartment where association with peptide takes place, 2) the efficiency of antigen capture and processing, and 3) association of class II molecules and peptides and recycling of the peptide-class II complexes to the cell surface. These factors will be discussed in turn. 


\section{Biosynthesis and transport of MHC class II molecules}

Upon translocation into the ER the $33-35 \mathrm{kD} \alpha$-chain and the $25-29 \mathrm{kD} \beta-$ chain of MHC class II molecules form a complex with a type II membrane glycoprotein, the invariant chain (Ii). The importance of invariant chain was demonstrated by Stockinger and colleagues in experiments where a fibroblast cell line transfected with I-E genes, and which expressed I-E but little Ii, was found to lack the ability to present low concentrations of an exogenous antigen until supertransfected with the invariant chain gene [16]. More recently it has been shown that the alternatively spliced $\mathrm{p} 41$ form of $\mathrm{Ii}$ is primarily responsible for this effect and that it may not be required for presentation of all antigens $[17,18]$. Ii is thought to act by targetting class II heterodimers to the endocytic pathway $[19,20]$ and contains a targetting signal for endosomes in its cytoplasmic domain $[19,21]$. In addition, association of a soluble form of Ii with Ia prevents binding of peptides in vitro [22] and is believed to protect the peptide binding groove during transit through the ER such that peptides are only bound in the endocytic compartment where Ia and Ii dissociate. An alternative possibility, not often mentioned, is that Ii does not prevent peptide interacting with the groove but keeps the Ia heterodimer in an "open" configuration so that peptide may bind but does not remain bound until Ii dissociates.

Morphological and biochemical evidence support the hypothesis that association with peptides occurs in an endocytic compartment where newly synthesised class II molecules and exogenous antigens (or peptides) are delivered. Guagliardi et al [23] noticed co-localisation of HLA-DR and endocytosed membrane receptors in early endosomes. Invariant chain was also seen in the same compartments but was not seen on the cell surface suggesting that the class II molecules were derived directly from the biosynthetic pathway [23]. Neefjes et al [24] concluded that newly-synthesised class II can be desialylated by endocytosed neuraminidase before reaching the cell surface, again suggesting the biosynthetic pathway for class II meets the endocytic pathway. Class II molecules could reach the endocytic compartment where association with peptide takes place either by being sorted directly to that compartment or by being sorted to the plasma 
membrane and being internalised by endocytosis. Although the experiments mentioned above are suggestive of the former hypothesis it is not yet possible to dismiss the possibility that class II molecules were sorted to the cell membrane but the rate of internalisation was too high to permit accumulation of Ii-associated Ia [23] or allow desialylation [24] at the cell surface.

\section{Antigen capture and processing}

Capture of antigens by accessory cells takes place by endocytosis or phagocytosis. ${ }^{1}$ Many cells can endocytose antigens non-specifically (pinocytosis) but, in addition, have specialised receptors that increase the efficiency of uptake for certain soluble antigens. B cells endocytose antigens that bind to membrane immunoglobulin and degrade the immune complexes intracellularly. Thus, $\mathrm{T}$ cell epitopes may be dependent on B cell epitopes because bound antibody protects its epitope from degradation [27]. This mechanism has been proposed to explain immunodominance in primary immune responses [27] but, in our view, is unlikely to be relevant in vivo since B cells cannot activate resting $T$ cells (see section 1.2). MØ have specialised receptors for immune complexes (Fc receptors), glycans (mannose receptor, $\beta$-glucan receptor) and complement (CR1, CR3, CR4) and all these are likely to participate in the acquisition of antigens by endocytosis and phagocytosis [28]. The ability of accessory cells to present exogenous antigens has long been thought to be related to their ability to internalise antigen (e.g. [29]). This view is challenged by observations on dendritic cells which are potent accessory cells and, yet, internalise very little antigen. Antigen acquisition by dendritic cells is reviewed in section 1.2.5.

Degradation of internalised ligand has traditionally been thought to take place in lysosomes [30]. However, recent evidence has demonstrated that early components of the endocytic pathway contain proteases $[31,32]$ and kinetic and sub-cellular fractionation studies have shown that antigen degradation can take place in endosomes [33] (see below).

\footnotetext{
1 Some authors refer to endocytosis as the general process of internalisation. Although that may be etymologically more correct, in this thesis the term endocytosis is reserved for the uptake of solutes via receptor mediated endocytosis or bulk-phase endocytosis (pinocytosis), in line with recent usage (c.g. references $[25,26])$
} 


\section{Association of MHC class II heterodimers and peptides}

There is much controversy as to where, along the endocytic pathway, newlysynthesised MHC class II molecules associate with peptides generated from exogenous antigens. A single compartment is thought to exist where degradation of antigen, proteolysis of invariant chain and peptide-Ia association takes place but this has been variously ascribed to an early or a late endosome (reviewed in [2]). For example, class II, invariant chain and plasma membrane markers can be found by immuno-electron microscopy in early endosomes containing cathepsins B and D [23] suggesting peptide loading of MHC class II takes place in these compartments. However, another morphological study (by a different group) localised endocytosed tracers and class II molecules to structures related to late endosomes [34]. Since none of these studies actually measured association between peptide and class II these data are difficult to interpret. Furthermore, it is possible that different accessory cells process antigen at different locations.

Another contentious issue is the role played by Ia recycling. Recycling of plasma membrane class II molecules through the endocytic pathway could, conceivably, lead to peptide exchange. Existing evidence suggests that different APC may behave in different ways. Cycloheximide treatment can inhibit presentation of exogenous antigens by Langerhans cells [35], by $\mathrm{M} \varnothing[36,37]$ and by some $\mathrm{B}$ cell hybridomas [38], suggesting that newly-synthesised class II heterodimers are essential for presentation. Davidson et al [39] directly measured association of radio-labelled antigen with class II molecules and concluded that naturally-processed peptide associates with newly-synthesised rather than recycling Ia in a B lymphoblastoid cell line. However, cycloheximide does not inhibit antigen presentation by B cell blasts [35] and peptide exchange has been demonstrated in viable APC [40]. Studies on a B cell line also suggest that immunogenic complexes are unstable and are lost rapidly $[36,37]$. For these cells, recycling of Ia through the endocytic pathway may allow peptide exchange but it is thought that this is an additional mechanism to peptide loading of newly-synthesised class II molecules [39]. 
An issue that has received little attention is how particulate antigens are processed and presented. Degradation of particles takes place in phagosomes and phagolysosomes. structures similar to but distinct from endosomes (see section 1.3.1). Presumably, either a pathway exists for class II heterodimers to reach phagosomes (early or late) or peptides generated in the latter must be transferred to the endocytic pathway. Interestingly "crosstalk" between the endocytic and phagocytic pathways has been recently demonstrated (see section 1.3).

Antigen presentation by accessory cells is commonly believed to result in $\mathrm{T}$ cell proliferation. One important aspect to emerge from the study of a specialised group of accessory cells, dendritic cells, is that $\mathrm{T}$ cell proliferation in primary immune responses requires two steps. In the afferent phase of the immune response $\mathrm{T}$ cells are sensitised against antigen in an MHC-restricted, antigen-specific way and proliferate and develop into $\mathrm{T}$ lymphoblasts capable of performing effector functions such as cytokine secretion or cytotoxicity. In the efferent phase of the immune response, activated $T$ cells recognise the antigen-MHC complex on the surface of any APC and respond by providing help or by lysing the APC and undergoing further clonal expansion. Over the last 19 years a large body of evidence has accumulated showing that, although any APC can present antigen during the efferent phase of an immune response, only dendritic cells or, possibly, B cell blasts, can act as accessory cells for the initiation of that response. The role of dendritic cells in antigen presentation is reviewed in the next section.

\section{2 - Dendritic cells}

Dendritic cells (DC) are potent accessory cells with high surface MHC class II expression and the capacity to activate $\mathrm{T}$ cells in an antigen-specific, MHC-restricted manner [41]. The distinguishing feature about DC is their unique ability to activate naive $\mathrm{T}$ cells as opposed to $\mathrm{T}$ cell blasts which can be activated by any accessory cell. The important functional distinction between the requirements to prime quiescent $\mathrm{T}$ cells, 
termed immunostimulation, and those to re-stimulate $\mathrm{T}$ lymphoblasts (antigen presentation) is illustrated in experiments using the mixed lymphocyte reaction (MLR) where alloreactive $\mathrm{T}$ cells are stimulated to proliferate by accessory cells bearing allogeneic MHC determinants. In a primary MLR using resting $\mathrm{T}$ cells, only DC and, to a lesser degree, $\mathrm{B}$ cell blasts have the capacity to induce significant levels of $\mathrm{T}$ cell proliferation. Both resting B cells and gamma interferon ( $\gamma$-IFN)-activated macrophages $(\mathrm{M} \varnothing)$ are totally inactive [42]. However, when the T cell blasts that develop in this MLR are re-stimulated with the same accessory cells, all of the latter can induce proliferation although DC still remain the most potent activators [42]. Memory $\mathrm{T}$ cells have the same requirement for stimulation by DC upon re-encounter with the antigen and, in this respect, behave like unprimed T cells [43]. Thus, the uniqueness of $\mathrm{DC}$ is in initiating de novo immune responses unlike other accessory cells (B cells, macrophages) which participate in amplifying those responses. This is further demonstrated by the finding that DC from mouse spleen pulsed with antigens in vitro before being injected into mice are the only spleen cells capable of priming $T$ cells in vivo in an antigen specific manner [44]. In addition, splenic DC have also been found to be the principal cells in mouse spleen carrying immunogenic peptides after injection of mice with antigen [45]. Thus, DC play a pivotal function in the generation of primary $\mathrm{T}$ cell responses against exogenous antigens. Accordingly, the T lymphoblasts induced in the course of such responses are mainly of the $\mathrm{CD}^{+}$type and are restricted to MHC class II molecules of the same haplotype as the presenting DC [44]. Less is known about the function of DC in immune responses against endogenous antigens involving $\mathrm{CD} 8^{+} \mathrm{T}$ cells. In vitro, $\mathrm{DC}$ are capable of activating $\mathrm{CD} 8^{+}$ T cells directly, obviating the need for $\mathrm{CD}^{+}{ }^{+}$help (although $\mathrm{CD}^{+}$helper cells cannot be excluded) and leading to the development of specific cytotoxic T lymphocytes (CTL) [4648]. DC are also potent antigen-presenting cells for viral antigens and generate specific CTL responses $[49,50]$. 


\subsection{1 - Tissue distribution}

Dendritic cells were originally described by Steinman and Cohn as a small subset of plastic-adherent cells in mouse spleen and other lymphoid organs [51]. The term DC, as employed then, referred to the isolated cells in vitro but over the years the term has broadened to include similar cells in situ and cells isolated from non-lymphoid tissues. The broader term "dendritic leukocyte" is sometimes used to designate this whole family of cells [52] but it has remained unpopular in the literature. We shall employ the terminology of Steinman [41] and refer to these cells as dendritic cells, qualifying it where necessary (e.g., splenic DC, epidermal DC). In addition, since the work described in this thesis has been performed exclusively with mouse DC we shall review primarily the features of murine DC although those of other species will be mentioned where relevant.

Dendritic cells form a vast network throughout the body and are present as trace populations in virtually every tissue with the possible exception of much of the brain [53]. They are bone-marrow derived cells and express the leukocyte common antigen, CD45. DC are typically recognised in situ by their highly distinctive dendritic morphology and constitutive expression of high levels of MHC class II antigens. In vitro the dendritic processes give rise to sheets or "veils" which are continuously being retracted and extended $[51,54]$. The motility of DC in culture is an exclusive property of this cell type and can be used to differentiate DC from other cells that can adopt a dendritic morphology, namely some cells of the myelomonocytic lineage. In the absence of markers exclusive to all dendritic cells, another deciding attribute of any dendritic cell is its immunostimulatory capacity. Thus, positive identification of DC requires that they be isolated from tissues for functional tests in vitro. It should be stressed that, although this has been carried out with DC from most lymphoid and some non-lymphoid tissues, the identity of other nonlymphoid cells as dendritic cells remains unproved by these strict criteria although they are commonly accepted to be part of the same lineage (see below). 


\section{Lymphoid dendritic cells}

Lymphoid organs, in particular, mouse spleen, have remained the traditional source of dendritic cells for in vitro studies. Present as a $1-2 \%$ of all nucleated spleen cells, procedures to enrich murine splenic DC typically involve fractionation over dense bovine serum albumin (BSA) as originally described by Steinman and Cohn [55] or over metrizamide gradients [56]. The low-density fractions obtained in this way can be further enriched for DC by plastic adherence and removal of non-adherent contaminating cells [57]. DC are only transiently adherent to plastic and detach after overnight culture whereas the bulk of $M \varnothing$ remain attached. Contaminating B cells and $M \varnothing$ in the overnight-cultured non-adherent population can be removed by rosetting with antibody coated sheep erythrocytes since DC do not express readily detectable Fc receptors.

Using similar procedures, DC have been isolated from most other lymphoid tissues $[41,57]$. DC from lymph nodes (LN) are very similar to splenic DC $[55,57]$. Peyer's patch DC, initially identified in cell suspensions [51] and in situ [58, 59], were isolated and characterised as potent accessory cells in $\mathrm{T}$ cell proliferative responses [60, 61]. Stimulation by isolated Peyer's patch DC was abrogated after depletion with 33D1[60], a cytotoxic antibody raised against splenic DC [62]. DC isolated from murine thymus, on the other hand, do not appear to express 33D1, are larger than their splenic counterparts and are optimally released following digestion of the tissue with collagenase [63]. These cells are capable of picking up antigens administered in vivo and presenting them to antigenspecific $\mathrm{T}$ cell clones, once more confirming their importance as accessory cells [64]. Similar thymic DC have also been obtained from human [65] and avian thymus [66]. Stimulatory DC have also been isolated from human tonsils [67, 68].

The in situ counterparts of isolated lymphoid DC have long been thought to be the interdigitating cells (IDC) of $\mathrm{T}$ cell areas $[69,70]$. In rodents, IDC have been identified in the periarteriolar lymphoid sheaths of spleen (PALS) $[59,71]$, paracortex of lymph nodes [59], thymic medulla [72] and T cell areas of Peyer's patch [58, 59]. These cells have 
cytological and phenotypic characteristics similar to isolated lymphoid DC [59] and when isolated splenic DC are injected intravenously into mice they home to the $\mathrm{T}$ cell-dependent area of spleen [73]. Similarly, radiolabelled DC isolated from rat afferent lymph and injected into the footpads of naive animals localise in the T cell area of draining LN and are indistinguishable from resident IDC by light or electron microscopy [74]. Thus, IDC appear to be the DC of lymphoid tissues. However, in murine spleen, isolated DC may come from the marginal zone and not be the same as IDC. A monoclonal antibody against isolated splenic DC, N418, labels cells both in the T cell areas of PALS tissue and nests of cells in the marginal zone [75]. In contrast, 33D1 labels only in the marginal zone [59], which is also the zone to which isolated splenic DC bind on frozen sections of spleen [73]. Moreover, only a small proportion of isolated splenic DC label with NLDC-145, a monoclonal antibody against interdigitating cells [70], and this population is 33D1- and does not express the heat stable antigen recognised by monoclonal J11d [63]. The majority of isolated splenic DC express the reciprocal phenotype, $33 \mathrm{D} 1^{+}, \mathrm{NLDC}-145^{-}, \mathrm{J1}^{+} \mathrm{d}^{+}$. It would seem, therefore, that there are two populations of splenic DC, the marginal zone DC $\left(33 \mathrm{D} 1^{+}, \mathrm{NLDC}-145^{-}, \mathrm{J} 11 \mathrm{~d}^{+}\right)$and the interdigitating cells of the $\mathrm{T}$ cell areas (33D1-, NLDC-145 $\left.{ }^{+}, \mathrm{J} 11^{-}\right)$. The majority of isolated splenic DC may be the marginal zone DC and possibly represent more immature cells which give rise to IDC (see below). In contrast, in thymus the great majority of isolated DC are $33 \mathrm{D} 1^{-}, \mathrm{NLDC}-145^{+}, \mathrm{J}_{11 D^{+}}$and may truly represent the in vitro counterpart of IDC [63].

\section{Non-lymphoid dendritic cells}

DC in non-lymphoid organs are difficult to identify in situ because of the lack of lineage specific markers and are often defined solely based on the fact that they are MHC class II (Ia)-expressing leukocytes with a dendritic shape that lack B cell (e.g. surface immunoglobulin) and certain M $\varnothing$ markers (e.g. acid phosphatase, non-specific esterase). Using these criteria, Hart and Fabre [53] identified interstitial DC in virtually every rat tissue except the bulk of the brain and proposed on the basis of their similarities to splenic 
DC that they may all be part of the same lineage ${ }^{2}$. More conclusive evidence has come from in vitro functional studies. DC have been isolated from rat [77] and murine lung [78], airway epithelia [79], intestinal lamina propria [61], and mouse hearts and kidneys (J. Roake and A.S. Rao, personal communication). All these cells are strongly Ia-positive, have the phenotypic characteristics of DC after culture (see below) and have potent immunostimulatory capacity, thus, fitting the functional definition of dendritic cells. Because of their immunostimulatory properties non-lymphoid DC are commonly thought to represent the passenger leukocyte responsible for sensitisation to allografts during transplantation [80]. The presence of DC passenger leukocytes in pancreatic islets has been directly implicated by the finding that pre-treatment of islet grafts with 33D1 and complement reduces their immunogenicity [81].

By far the best characterised non-lymphoid DC is the Langerhans cell (LC) of the epidermis [82]. LC are present in other epithelia in addition to epidermis (e.g. buccal mucosa, genito-urinary epithelium), express high levels of MHC class II and possess Birbeck granules (BG), a characteristic organelle that is conceivably associated with their antigen processing ability [82]. Langerhans cells are discussed in greater detail below.

\section{Dendritic cells in circulation}

Blood is the main source of human DC $[54,83-85]$ and DC isolated from blood are similar to lymphoid DC possessing immunostimulatory activity. Mouse blood also contains a recently identified population of proliferating dendritic cell precursors [86].

DC are also present in afferent but not efferent lymph of rabbit [87, 88], rat [89], mouse [90, 91], humans [92] and sheep [93-96]. In rats, pseudoafferent lymph [89], obtained by cannulation of the thoracic duct after mesenteric node lymphadenectomy, is commonly used as the source of DC since rat splenic DC do not adhere to plastic unlike their mouse counterparts. DC from lymph express Ia and are called veiled cells (VC). In

2 Intriguingly, a recent report suggests that microglia in normal human brain express HLA-DR and CD1a constitutively and are phenotypically closer to Langerhans cells than to traditional resident macrophages [76]. 
culture, such cells closely resemble DC isolated from lymphoid organs, continuously extending and retracting veils but, like LC, are occasionally found to contain Birbeck granules [88].

\subsection{2 - Migration}

It is clear from the above that DC comprise a vast network of accessory cells specialised for $\mathrm{T}$ cell responses. The exact inter-relationships between different DC are not fully understood but it is believed that lymphoid DC originate from non-lymphoid DC which in turn arise from bone-marrow precursors [97]. Thus, two main migrational pathways are thought to exist, one from the bone-marrow to non-lymphoid tissues which leads to seeding of these tissues with MHC class II-positive DC, and another pathway from non-lymphoid to lymphoid tissues giving rise to IDC (and, possibly, DC of the marginal zone in spleen). Very little information is, as yet, available about the former pathway but support for the latter has been accumulating steadily and is summarised below.

\section{Migration via the lymph}

Much indirect evidence has accumulated from studies on contact sensititvity suggesting that LC can migrate to draining lymph nodes via the lymph (as veiled cells) and present contact sensitisers to T cells. Cells morphologically similar to LC and containing Birbeck granules were initially identified in electron microscopical studies of LN draining sites where antigen had been injected [98]. In experiments where mice were skin-painted with contact sensitisers a gradual accumulation of antigen-bearing DC was also seen in draining LN [99-101]. These DC also often contained Birbeck granules [101] and/or were $\mathrm{Ia}^{+}[102]$, suggesting that they were probably derived from Langerhans cells. However, none of these studies exclude the possibility that antigen draining freely to lymph nodes induces the formation of Birbeck granules in resident local $\mathrm{Ia}^{+} \mathrm{DC}$. These objections were partly addressed in a study by Kripke and colleagues [103] who grafted C3H ( Iak) skin 
onto BALB/c (Iad) nude mice. Application of fluorescein isothiocyanate (FITC) to the graft led to an accumulation of cells in draining $\mathrm{LN}$ that could transfer contact sensitivity to FITC to naive $\mathrm{Ia}^{\mathrm{k}}$ but not $\mathrm{Ia}^{\mathrm{d}}$ recipients proving that the sensitising cell(s) must have come from the graft. Unfortunately, the authors did not show that this cell derived from a Langerhans cell.

Movement of LC to LN via the lymphatics has also been suggested by other observations. Larsen et al [104] observed a rapid loss of LC from mouse epidermis in transplanted skin or explants placed in organ culture. The LC next appeared in "cords" in the dermis, presumed to be LC in lymphatics, before leaving the skin. LC leaving the skin explants in organ culture could be collected at the bottom of the culture vessel and are presumed to represent cells that would have reached the lymph nodes were the lymphatics not severed.

Veiled cells injected into footpads of rats reach the draining LN via the lymph [74] but, interestingly, radiolabelled LC do not migrate into mesenteric or peripheral lymph nodes when injected intravenously. Instead, injected LC migrate mostly to skin and, when the infusion is performed across a major histocompatibility barrier, can be seen in both dermis and epidermis by staining with antibodies against MHC class II molecules of donor haplotype [105]. However, skin taken from mice infused with haptenated epidermal cells transfers contact sensitivity when grafted onto naive syngeneic animals suggesting that the infused LC may move to LN after reaching the skin, like resident LC [105]. This pattern of LC migration may be altered in pathological conditions. Kaplan et al [106] have suggested that in the course of a delayed-type hypersensitivity response, LC in the epidermis of leprosy patients move upwards and are sloughed off the surface rather than migrate downwards into the dermis. The significance of these observations is not understood.

\section{Migration via the blood}

Work from Austyn and colleagues has identified another migratory pathway by which non-lymphoid DC may reach the spleen [73, 107]. Radio- or fluorochrome-labelled 
DC injected intravenously into mice home specifically to the $T$ cell areas of white pulp and are not found in peripheral or mesenteric lymph nodes (although they may be able to move from the liver into coeliac LN [74]). Homing of DC to spleen has been confirmed in a rat model $[74,108]$. This experimentally-induced migration via the blood is thought to be analogous to the migration of DC from non-lymphoid vascularised organs to the spleen. Larsen et al [109] showed that $\mathrm{Ia}^{+}$leukocytes resident in hearts (thought to be interstitial heart DC [110]) are greatly reduced in number following allografting. Cells expressing Ia of the donor haplotype can subsequently be found in the spleen of the recipient, associated with $\mathrm{CD} 4+\mathrm{T}$ cells but, in contrast to the experiments described before, this time in the peripheral white pulp (B cell area) of spleen. The reason for the different localisation of graft-derived versus injected DC is not known but is, conceivably, an artefact from the allograft model: allo-DC that migrate to $\mathrm{T}$ cell areas of spleen may be killed by local cytotoxic responses [111].

Migration of DC via the blood must also occur from the bone marrow to nonlymphoid tissues. DC from lymphoid tissues are non-replicating and originate from a bone marrow proliferating precursor (reviewed by Bowers and Goodell in reference [112]). Very little is known about DC precursors except that they are Ia- and are present in cultures of rat bone marrow [113] and, possibly, in human bone marrow and blood [114]. Recently, Inaba and colleagues [86] succeeded in characterising a proliferating Ia-negative DC precursor found in mouse blood. In culture, in the presence of granulocyte/macrophagecolony stimulating factor (GM-CSF), it gives rise to progeny $\mathrm{DC}$ which are potent stimulators of the MLR and home to T cell areas of LN when injected into the footpads of mice. G-CSF or M-CSF did not cause the formation of DC providing evidence that these cells constitute a distinct pathway of myeloid development. Similar precursors are now being characterised in murine bone marrow (R. M. Steinman, personal communication). However, the blood DC that are commonly isolated from human blood are thought to represent DC in transit from non-lymphoid to lymphoid organs rather than DC travelling from bone marrow because they are indistinguishable from lymphoid DC unlike the Ia- 
DC precursors.

In summary, DC are thought to be transient, non-replicating populations en route from one tissue to the next. The flux of cells is supported by replicating bone-marrow DC precursors that travel via the blood to seed non-lymphoid tissues although it is possible that some migrate directly to lymphoid tissues. Non-lymphoid DC migrate to lymphoid tissues via the lymph and/or blood. The fate of lymphoid DC is poorly understood. Turnover of DC in spleen (and in rat lymph [89]) is rapid, with a half-life of less than 1 week [115]. Unlike lymphocytes, DC are not thought to recirculate since no DC are detectable in efferent lymph. Instead, it is commonly believed that IDC are the putative terminal stage of the DC system and die in situ, being disposed of by local scavengers (reviewed by Fossum in reference [112]). The presence of immunostimulatory DC in nonlymphoid tissues and their migration to the $\mathrm{T}$ lymphocyte areas of lymphoid tissues makes them ideal passenger leukocytes involved in the sensitisation against allografts $[80,116]$.

\subsection{3-Maturation}

Much evidence has accumulated from the study of murine Langerhans cells in vitro, that DC can exist in distinct maturational states, each with unique functional and phenotypic characteristics [82]. Cultured LC and the traditionally isolated splenic DC are virtually indistinguishable in their functional and phenotypic properties but differ from freshly-isolated LC and LC in situ. These differences are discussed below.

\section{Phenotypic maturation}

The phenotype of DC isolated from mouse spleen and studied after overnight culture, referred to by some workers as the "Steinman cell" [53], has often been taken as the standard phenotype against which any putative new element of the DC family should be compared. These DC are non-phagocytic cells that constitutively express high levels of MHC class II and class I antigens, the leukocyte common antigen CD45, lack certain mouse M $\varnothing$ markers (Fc receptors, F4/80), B cell (surface immunoglobulin, B220) and T 
cell (T cell receptor, Thy-1) markers, express low levels of C3bi receptor (CD1 1b/CD18 or CR3) and IL-2 receptor $\alpha$-chain (p55) and express certain DC-restricted markers recognised by monoclonals 33D1 or NLDC-145 (see above), N418 (CD11c/CD18) and. more recently, M342 $[41,62,63,75,117,118]$ (table 1.1). However, it is clear that other cells that are considered to be part of the DC system do not have all the features of the "Steinman cell". Interdigitating cells, thymic DC and Langerhans cells do not express 33D1 and express NLDC-145 unlike the majority of isolated splenic DC ([63]; see above). Although extensive phenotyping of non-lymphoid cells is yet to be performed, again some discrepancies are apparent. Lung DC express NLDC-145 like thymic DC and LC but are heterogeneous and some also express $\mathrm{Fc} \mathrm{R}^{3}, \mathrm{~F} 4 / 80, \mathrm{IL}-2$ receptor $\alpha$-chain and higher levels of CR3 than splenic DC [78]. Putative DC freshly isolated from hearts or kidneys do not express NLDC-145 but express F4/80 weakly, FcyR and M1/70 (J. Roake and A. S. Rao, personal communication).

A possible reason for these discrepancies has been suggested by the study of Langerhans cells in vitro. Freshly isolated LC and LC in situ are markedly different from isolated splenic DC and express F4/80 (mouse), FcyR (FcyRII in human), NLDC-145, CD1a (human), membrane ATPase and nonspecific esterase (NSE), in addition to MHC class II antigens and CR3 [82]. In contrast to splenic DC, LC do not express 33D1 or N418. However, when murine LC in bulk epidermal cell suspensions are cultured for 1-2 days they undergo profound phenotypic changes and come to resemble typical "Steinman cells" [120]. Within the first $12 \mathrm{~h}$ of culture there is marked synthesis of Ia and invariant chain with the result that MHC class II antigens are markedly up regulated $[35,121]$. The cells become larger and more veiled (in contrast to the "hairy" appearance of freshly isolated LC), Fc receptors are lost within the first $24 \mathrm{~h}$ of culture and F4/80 is no longer detectable after 2 days [120]. Membrane ATPase, NSE and Birbeck granules are also

\footnotetext{
${ }^{3} \mathrm{Fc} \gamma$ receptor expression in mouse $\mathrm{DC}$ has been defined primarily on the basis of staining with the $2.4 \mathrm{G} 2$ monoclonal antibody against Fc $\gamma R$ II but which also recognises Fc $\gamma R$ III, previously known as Fc $\gamma R$ II $\alpha$ (see section 1.3.2). It is not known which gene(s) is expressed in DC. In line with the new terminology [119] we

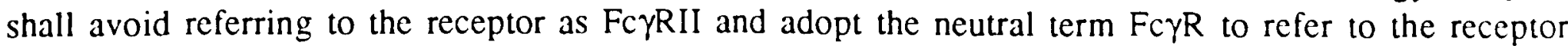
recognised by $2.4 \mathrm{G} 2$ in mouse dendritic cells.
} 
absent from cultured LC [120] but, in turn, N418 (but not 33D1) expression is up regulated (personal observations) together with $\mathrm{B} 7 / \mathrm{BB} 1$, the ligand for CD28 [82, 122]. Similar changes have been reported for human LC: Birbeck granules and membrane ATPase activity were greatly reduced, NSE, Fc $\gamma$ RII (CDw32) and CD1a expression were lost and CD40, ICAM-1 (CD54) and IL-2 receptors (CD25) were induced [123, 124]. Thus, with the exception of 33D1 antigen expression in mouse, cultured mouse and human LC are indistinguishable from isolated splenic DC and human blood DC, respectively (see table 1.1 for summary on murine DC). This transformation in culture is part of a process termed maturation [125]. 
Table 1.1: Phenotype of murine dendritic cells

Marker/Antigen

Fresh LC Cultured LC Splenic DC

MHC products

class $\mathrm{I}(\mathrm{H}-2 \mathrm{~K})$

class II (H-2I)

Leukocyte markers

Leukocyte common

CD45

antigen

$\mathrm{T}$ cell markers

$\mathrm{CD} 8^{*}$

$\mathrm{CD} 4 \S$

Thy-1

TcR

B cell markers

B220 (CD45RA)

J11d

MØ markers

F4/80

Membrane ATPase

Non-specific esterase

DC markers

33D1

NLDC-145

N418 (CD1 1c)

Adhesion molecules

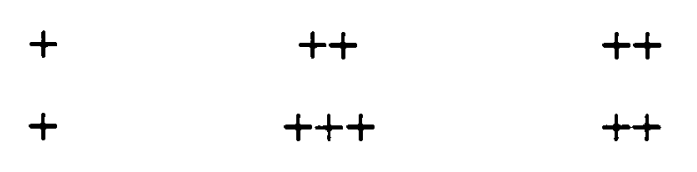

$+\quad+\quad+$

$+$

$\begin{array}{ccc}- & - & - \\ - & - & - \\ - & - & - \\ - & - & - \\ - & - & +1-9 \\ + & + & -8 \\ + & - & - \\ + & - & - \\ + & - & +1-9 \\ - & - & +1-9 \\ + & + & + \\ - & + & \end{array}$

LFA-1 (CD11a/CD 18)

ICAM-1 (CD54)

$\mathrm{B} 7 / \mathrm{BB} 1$

\section{Receptors}

IL-2 receptors

p55 (CD25)

Fc receptors

Fc $\gamma$ RII (RIII?)

Complement receptors

CR3 (CD11b/CD18)

$\begin{array}{lll}- & + & + \\ + & + & + \\ - & + & +\end{array}$

Not an exhaustive list. The phenotype of splenic DC refers to cells after overnight culture isolated using the conventional procedure. See notes below and text for more details. References: $[63,82,117,122,126,127]$ and personal observations.

* Expressed in a subpopulation of splenic DC.

$\$$ Expressed in a subpopulation of splenic DC before overnight culture.

Expressed in reciprocal populations of splenic DC. See text for discussion.

$¥$ Recognised by monoclonal $2.4 \mathrm{G} 2$. See text for details.

₹ Expressed at low levels. 


\section{Functional maturation}

Maturation of LC in culture also leads to profound changes in LC function. Schuler and Steinman [120] found that although freshly-isolated LC expressed a high level of MHC class II antigens they were poor stimulators of $\mathrm{T}$ cell proliferation in the MLR and oxidative mitogenesis (polyclonal proliferation of periodate-treated $\mathrm{T}$ cells) assays. However, cultured LC in these assays were even more potent stimulators than splenic DC, on a per cell basis [120]. This acquisition of immunostimulatory ability during culture is not thought to be due to up regulation of Ia since both fresh and cultured LC stimulated $\mathrm{T}$ cell blasts to the same extent and $12 \mathrm{~h}$-cultured LC (which still have the functional properties of fresh LC) and 60h-cultured LC (mature LC) express equivalent amounts of Ia [128]. Neither is it an artefact induced by the trypsin treatment used to isolate LC because trypsinisation of mature LC does not reduce their immunostimulatory activity [120, 128]. Instead, it may be related in part to the fact that cultured but not fresh LC are able to cluster with $T$ cells [120] in an antigen independent fashion [128] as has been shown for splenic DC [129]. Romani et al [130] confirmed that cultured murine LC and splenic DC were potent stimulators of the allo-MLR but noted that they were poor presenters of intact myoglobin to MHC class II-restricted myoglobin-specific T cell clones. Fresh LC, on the other hand, were efficient presenters of intact protein and all three populations presented myoglobin peptides. These results have been confirmed with different clones and with primed lymph node $T$ cells $[35,131]$. In addition, Puré et al [35] showed that after exposure to intact antigen LC can retain peptide-MHC complexes for presentation to $\mathrm{T}$ cells for at least 2 days. Therefore, it appears that cultured LC have lost the ability to process native antigens while acquiring the ability to prime $\mathrm{T}$ cells against peptides generated at an immature stage. Thus, a functional dichotomy exists between mature and immature LC. Fresh LC are capable of processing exogenous antigens and generating the appropriate MHC class II-peptide complexes that are displayed in an antigenic form on the cell surface. Mature DC can display these antigens in an immunogenic form that activates antigen-specific resting $T$ cells but cannot generate new antigenic complexes. It should be 
mentioned, at this stage, that somewhat conflicting results have been obtained by a separate group of investigators. These workers reported that cultured $\mathrm{EC}$ from $\mathrm{C} 3 \mathrm{H}\left(\mathrm{H}-2^{\mathrm{k}}\right)$ mice were more potent than fresh EC at stimulating an antigen-specific $\mathrm{T}$ cell hybridoma [132] when pulsed with native antigen. These discrepancies were subsequently attributed to MHC differences by Aiba and Katz [131]: it appears that cultured $\mathrm{H}-2^{\mathrm{k}} \mathrm{LC}$ may be capable of presenting native proteins unlike the $\mathrm{H}-2^{\mathrm{d}} \mathrm{LC}$ used by Romani and colleagues [130]. However, in a recent review, Romani and Schuler [82] state that "using the same T cell lines as Aiba and Katz ... in each case fresh $L C$ are far superior to cultured $L C$ even though the latter may have some residual processing capacity for some antigens". Methodological differences, namely the use of purified LC versus epidermal cells may be behind some of these discrepancies.

The mechanisms underlying the loss of antigen-processing ability are not entirely clear. Work from the group of Romani and Schuler has shown that antigen-processing by fresh LC is sensitive to both chloroquine and monensin and that loss of acidic vesicles from the endocytic pathway accompanies down regulation of antigen-processing capacity during LC culture [133]. However, other studies have shown that there is no difference in accumulation of acridine orange, a lysosomotropic fluorochrome, in fresh and cultured human LC [134] although the same workers had previously noted that fresh but not cultured LC could internalise surface HLA-DR cross-linked with antibodies into acidic compartments [135]. It is possible that acridine orange does not have access to a pool of acidic vesicles that is down regulated in culture and where antigen processing occurs. A major contributing factor to the changes in antigen-processing capacity is thought to be the rate of MHC class II synthesis. Fresh LC synthesise large amounts of Ia and Ii but synthesis effectively ceases by $24 \mathrm{~h}$ of culture $[35,121,136]$. The shutdown in class II synthesis may prevent the generation of new peptide-Ia complexes since antigen presentation by fresh LC can be markedly reduced by preventing up regulation of Ia with cycloheximide during the antigen pulse [35]. 


\section{$\underline{\text { Regulation of LC maturation }}$}

Maturation of Langerhans cells in culture is regulated by cytokines. When fresh LC purified from EC suspensions are cultured in fresh medium viability drops markedly unless keratinocyte conditioned medium (KCM) is added back to the cultures [137-140]. Keratinocytes are able to synthesise a variety of cytokines, including $\mathrm{M}_{-}, \mathrm{G}-$ and $\mathrm{GM}_{-}$ CSF, IL-1 $\alpha$, IL-6 and IL-8 [141]. Using stimulation of the MLR as an assay for LC maturation, GM-CSF was found to be the active cytokine in KCM: when pure GM-CSF was added to fresh LC for 3 days they became potent simulators and anti-GM-CSF could prevent KCM-induced maturation [137]. IL-1 $\alpha$ was shown to synergise with GM-CSF in enhancing LC function using a different proliferative assay (oxidative mitogenesis) [138]. ${ }^{4}$ Other cytokines may also exert a synergistic effect since LC cultured in KCM are functionally more mature than those cultured in a combination of GM-CSF/IL-1 (F. Koch and E. Kämpgen, personal communication). Indeed, TNF- $\alpha$ and IL- 6 up regulate HLADR and CD1a in human gingival LC [142].

Functional maturation induced by GM-CSF is not simply a result of increased viability. LC cultured with TNF- $\alpha$ are viable and yet do not stimulate the MLR [139]. Recent data, however, suggests that, unlike LC cultured in GM-CSF, fresh LC pulsed with antigens and cultured in TNF- $\alpha$ lose their immunogenic peptides; thus, the lack of activity in the MLR may be due to these LC not being able to present antigen to alloreactive $\mathrm{T}$ cells (F. Koch, personal communication).

\section{Maturation as a feature of DC}

Since freshly-isolated LC are the prototype non-lymphoid dendritic cells it might be expected that other non-lymphoid DC undergo a maturational process in culture similar to that observed with LC. To date this is not clear. DC isolated from non-lymphoid organs do not have the typical phenotype of fresh LC although the lengthy procedures typically

\footnotetext{
${ }^{4}$ High affinity IL-1 receptors have recently been shown to be expressed in murine LC, and are down regulated during culture (E. Kämpgen, personal communication).
} 
involved in isolation could lead to maturational changes; these changes could also explain some of the heterogeneity typically found in non-lymphoid DC populations in vitro [79]. Recent data from our laboratory, however, suggests freshly-isolated heart and kidney DC undergo a maturational process in culture similar to LC (J. Roake, A. S. Rao et al, in preparation). During overnight culture, isolated heart DC appear to down regulate F4/80, FcR and M1/70 although expression of these markers is not completely lost. A less pronounced down regulation is sometimes also found with kidney DC which, consistently, up regulate MHC class II expression in culture. Furthermore, kidney DC cultured overnight but not freshly-isolated are potent stimulators of oxidative mitogenesis. Thus, at present, our bias is that non-lymphoid DC, like LC, are in an immature state. Improvement in methods of isolating non-lymphoid DC and definition of the culture conditions required for DC maturation will provide clearer answers.

Since isolated splenic DC have the phenotype of cultured LC it has been assumed that lymphoid DC are functionally and phenotypically mature DC that have immigrated from non-lymphoid tissues via the lymph and blood. Thus, the terms mature DC and lymphoid DC have often been used synonymously [116]. However, isolated lymphoid DC have traditionally been studied after overnight culture. Recent evidence suggests that before this culture step, at least a subset of isolated splenic DC are, in fact, immature and have many of the features of Langerhans cells. Fresh splenic DC were found to process exogenous antigens and to express F4/80 and FcyR (table 1.1, footnotes); these features were lost after overnight culture while the cells became more potent stimulators of the $\operatorname{MLR}[44,126,143] .{ }^{5}$ Furthermore, GM-CSF was crucial in maintaining the viability of freshly-isolated sorted DC and also potentiated their stimulatory function [126]. It is likely, therefore, that DC that are isolated from spleen correspond to a population of recently immigrated cells from non-lymphoid organs in the marginal zone (see also section 1.2.1). Functional maturation of these cells may, therefore, accompany their relocation

\footnotetext{
5 As for LC, the loss of antigen-presenting capacity during functional maturation of splenic DC may differ between mouse strains (see above). A report just published [144] shows that cultured splenic DC from $\mathrm{H}-2^{\mathrm{k}}$ mice are able to process and present hen egg lysozyme.
} 
from that area into the $\mathrm{T}$ cell areas of PALS where they become IDC. A recent report from R. Steinman's group gives support to this hypothesis [118]. The authors describe a new hamster monoclonal, M342, directed against an intracellular antigen on IDC and B cells, which labels IDC in the white pulp but not DC in the marginal zone of spleen. Freshly isolated splenic DC do not stain with M342 but start expressing the antigen after overnight culture [118].

In summary, maturation appears to be a characteristic of DC, both in lymphoid and non-lymphoid tissues. Immature DC share M $\emptyset$ markers such as F4/80 and Fc $\gamma R$ and are capable of processing exogenous antigens for presentation but have poor immunostimulatory activity. Mature DC have lost such markers and the ability to present whole proteins but present peptides efficiently and are very potent stimulators for $\mathrm{T}$ cells (table 1.2). As such, immature DC can be said to make antigenic peptides whereas mature DC present those peptides in an immunogenic form. GM-CSF promotes DC maturation and other cytokines such as $\Pi$ L-1 may act synergistically. So far, most of the work supporting this model has been done in the murine system using Langerhans cells as a model for non-lymphoid DC. These observations will need to be expanded to other species and other non-lymphoid DC in the near future.

Table 1.2: Functional properties of murine dendritic cells and macrophages

\begin{tabular}{ccccc}
\hline & Fresh LC & Cultured LC & Splenic DC & M \\
\hline Antigen processing & +++ & $+/-$ & $+/$ & +++ \\
Antigen presentation & & & & \\
(sensitised T cells) & ++ & +++ & +++ & ++ \\
$\begin{array}{c}\text { Immunostimulation } \\
\text { (naive T cells) }\end{array}$ & - & +++ & +++ & - \\
\hline
\end{tabular}

Adapted from reference [97]. 


\subsection{4 - T cell activation}

What is the nature of the potent immunostimulatory capacity of dendritic cells? Despite an intensive search, the unique signal provided by $\mathrm{DC}$ in $\mathrm{T}$ cell activation remains elusive. DC do not produce IL-1, a cytokine that is crucial in antigen presentation by MØ. Study of the interactions between $\mathrm{T}$ cells and DC in in vitro assays (antigen-specific proliferation, MLR, oxidative mitogenesis, anti-CD3 mitogenesis) have placed much importance of the initial step that leads to the formation of $\mathrm{T}$ cell-DC aggregates or "clusters" [128, 129, 145-147]. T cell-DC clustering precedes and is essential for successful priming (responsiveness to IL-2) and proliferation [146]. Inaba and colleagues [145] demonstrated that clusters of anti-CD3 coated T cells and LC placed on one side of a semi-permeable filter are not capable of inducing the proliferation of anti-CD3 coated T cells placed on the other side although $\mathrm{T}$ cells in the clusters proliferate and IL-2 is produced and diffuses freely across the barrier. Similarly, $\mathrm{T}$ cells do not proliferate outside the clusters [146]. These results suggest that cognate interaction between DC and T cells, rather than a soluble factor, are necessary for $\mathrm{T}$ cell activation. It is conceivable, however, that a soluble factor for activation is required at locally high concentrations that can only be achieved within the cluster itself. The clustering phenomenon, itself, offers some clues to the mechanism of activation. T cell blasts can cluster with $\mathrm{DC}$, fresh $\mathrm{LC}, \mathrm{M} \emptyset$ or $\mathrm{B}$ cells in an antigen-dependent way at $37^{\circ} \mathrm{C}$ and $4{ }^{\circ} \mathrm{C}$. Of these accessory cells, only DC are capable of clustering in an antigen-independent way and only at $37^{\circ} \mathrm{C}$ [129]. These and other results have been tentatively interpreted to suggest that DC are capable of "surveying" a large number of $\mathrm{T}$ cells through antigen non-specific clustering until the appropriate T cell receptor reactivity is found. Presumably, at that stage, these cells lose the capacity to form antigen-independent clusters and activation ensues. The unique features of DC (dendritic processes, motility) make them ideally suited for this type of interaction [148].

Little is known about the identity of adhesion molecules involved in DC-T cell clustering. DC express high levels of ICAM-1 and LFA-3 and these are likely to interact 
with the corresponding ligands on T cells, LFA-1 and CD2, respectively [41]. Stimulation of the MLR by LC can be markedly inhibited with antibodies against CD11a, CD18 and ICAM-1, particularly if used in combination [149]. Inhibition with anti-CD18 [147] and anti-CD11a [68] is likely to be due to LFA-1 on both DC and T cells [150]. In contrast, inhibition by anti-LFA-3 occurs at the level of the DC $[68,150]$. It has also been suggested that interaction between DC and $\mathrm{T}$ cells is facilitated by the low level of sialylation of class I and class II MHC molecules on DC [14, 150, 151] although other workers have failed to find evidence for differential glycosylation of class II molecules in LC [121]. One molecule that plays a key role in T cell activation is CD28 which is expressed on most $\mathrm{CD}^{+}$and $\approx 50 \% \mathrm{CD}^{+} \mathrm{T}$ cells [152]. B7/BB1, the ligand for $\mathrm{CD} 28$ (and for a homologous molecule, CTLA-4), has recently been found on mature LC and splenic DC but not on fresh LC [122]. The precise role of this and other DC molecules in immunostimulation awaits further investigation.

\subsection{5 - Acquisition of antigens by $D C$}

There is debate as to whether immune responses are initiated peripherally, in nonlymphoid tissues, or centrally, in lymphoid tissues. The study of dendritic cells has favoured the latter hypothesis $[97,116]$. For peripheral sensitisation, the probability of an antigen-specific $\mathrm{T}$ cell (of $10^{12} \mathrm{~T}$ cells estimated in humans) encountering a peptide-MHC complex that matches its specificity must be exceedingly small. A T cell would have to travel through the circulation and across the endothelium into every extravascular space, eventually to meet a dendritic cell presenting the antigen and initiating a response. The biology of the dendritic cell network appears to be specialised to overcome this difficulty. Migration of non-lymphoid DC into lymphoid tissues (where over $90 \%$ of $\mathrm{T}$ cells are located at any one time) appears to provide an efficient way of maximising the probability of a successful encounter with antigen. Furthermore, mature DC are motile, can "survey" " $T$ ' cells independently of their reactivity, have processes that allow intimate contact with $\mathrm{T}$ cells and have the unique ability to stimulate the antigen-specific $\mathrm{T}$ cell once specific 
reactivity is achieved. On the other hand, immature DC in non-lymphoid tissues are capable of processing antigens and presenting them to recruited, activated $\mathrm{T}$ cells in the course of an immune response but do not stimulate virgin $\mathrm{T}$ cells such that responses are initiated centrally. The loss of antigen-processing ability and shutdown of class II synthesis may have evolved to ensure that LC retain antigens encountered in situ and that they do not become loaded with irrelevant peptides en route to lymphoid tissues. Implied in this model is the concept that immature DC function as sentinels of the immune system, acquiring antigens and transporting them to $\mathrm{T}$ cell areas of lymphoid tissues for presentation. Thus, the functional dichotomy between immature DC and mature DC (table $1.2)$ is thought to extend to antigen acquisition: immature DC acquire antigens and mature DC present those antigens. Surprisingly, however, very little direct evidence exists for the capacity of DC to handle antigens. In this section we review the ability of lymphoid and non-lymphoid DC to acquire antigens for presentation.

\section{Antigen acquisition by splenic DC}

Internalisation of antigens can occur via endocytosis (soluble antigens) or phagocytosis (particulate antigens). However, lymphoid DC and veiled cells in lymph have traditionally been described as non-phagocytic and poorly endocytic in vitro $[55,89]$ and in vivo $[59,71,153]$. Such data would seem to be at odds with the view that antigen presentation is directly related to the ability of accessory cells to internalise the antigen. For example, presentation of limiting concentrations of antigen by FcR-bearing B cells and $M \varnothing$ is enhanced if the antigen is administered as immune complexes; presentation by the latter cell type is enhanced even further if these complexes are conjugated to latex beads [29]. However, in contrast to these accessory cells, the exogenous antigen presentation pathway of DC may be specialised to handle small amounts of antigens very efficiently so that the ability of DC to internalise antigens ceases to be a limiting factor [41]. Recent data showed that freshly isolated splenic DC pulsed with antigen during (but not after) overnight culture, could prime T cells in vivo in a MHC-restricted fashion when injected at a dose as small as $3-8 \times 10^{4}$ into the footpads of mice [44]. Spleen or peritoneal 
cells did not have this capacity although peritoneal $\mathrm{M} \varnothing$ were able to take up far more antigen than DC during the pulsing period. In the converse experiment, antigen was injected intravenously into mice and isolated accessory cells were tested for stimulation of antigen-specific $\mathrm{T}$ cell clones. In thymus [64] or spleen [45], only $\mathrm{DC}$ were found to contain antigen in an immunogenic form. These observations imply that DC internalise and degrade antigens but do not formally rule out the hypotheses that processing is extracellular [154] or that processing is carried out by a co-operating cell (e.g. MØ) which transfers peptides to DC. Arguing against these hypotheses is the observation that small amounts of antigen can be endocytosed by DC $[44,155]$ and that antigen processing by DC is sensitive to lysosomotropic agents, implying the involvement of a low pH intracellular compartment $[35,44,133,144,156]$. In addition, no evidence for peptide regurgitation by $\mathrm{M} \varnothing$ was found in experiments where $\mathrm{H}-2^{\mathrm{k}} \mathrm{M} \varnothing$ pulsed with myoglobin were mixed with mature $\mathrm{H}-2^{\mathrm{d}} \mathrm{DC}$ and failed to stimulate a $\mathrm{I}-\mathrm{E}^{\mathrm{d}}-$ restricted myoglobin-specific $\mathrm{T}$ cell clone [45] (although it is theoretically possible that $\mathrm{H}-2^{\mathrm{k}} \mathrm{M} \emptyset$ produce peptides that cannot bind to $I-E^{d}$ if processing by the two strains is different). While all of the work reviewed so far pertains to exogenous antigens being presented on MHC class II molecules, Nair et al [157] have reported introduction of exogenous protein into the class I presentation pathway of DC through pH-sensitive liposomes showing that, like other cell types [158], DC have the capacity to generate class I-restricted responses against exogenous antigens provided these are given access to the cytosol.

A striking finding has been that splenic DC pulsed with antigen in vitro (during overnight culture) can generate antibody responses in vivo as efficiently as conventional immunisation with adjuvants [159]. This is surprising because until now DC have not been thought to present antigen to B cells, that function being associated with an unrelated type of cell, the follicular dendritic cell. However, because mice in those experiments were later boosted with soluble antigen [159], it is possible that B cell priming only occurred at that stage. 
Immature DC in the periphery as sentinels of the immune system

The fact that only splenic DC pulsed with antigen before overnight culture elicited a response in vivo (see above) agrees with in vitro results on DC maturation and suggests that acquisition of antigens is part of the immature DC phenotype. In line with this hypothesis, DC isolated from rat lung following exposure to aerosolised ovalbumin were capable of presenting this antigen to immune $T$ cells [77]. Likewise, rat veiled cells from pseudo-afferent lymph draining the gut wall present antigens administered intraintestinally [160]. Much suggestive evidence for the idea that immature DC acquire and transport antigen to lymphoid tissues came initially from in vivo observations on LC and their migratory form in the lymph, the veiled cell (VC). Mouse veiled cells travelling in the lymph from non-lymphoid to lymphoid tissues are also immature in being able to process intact antigen in vitro for presentation [91]. Early reports implicated skin LC [161] and afferent lymph cells $[162,163]$ in the induction of contact sensitivity. Silberberg-Sinakin et al [98] injected ferritin intradermally into guinea pigs and found ferritin inside cells with Birbeck granules in draining lymph nodes. Whole LN cell suspensions from immunised animals could transfer delayed-type hypersensitivity to ferritin when injected into naive syngeneic guinea pigs. Similarly, experiments using mice skin-painted with contact sensitising fluorochromes have demonstrated an influx of labelled dendritic cells into draining lymph nodes which could stimulate syngeneic $T$ cells $[100,101]$ and could adoptively sensitise normal animals $[100,103]$. In contrast, thy $-1^{+}$epidermal cells $(\gamma / \delta \mathrm{T}$ cells) which were also found in the LN were not visibly associated with antigen [102]. It is important to note that none of these experiments rule out the hypothesis that antigen drained to the LN and was picked up locally by LN DC and, as such, do not constitute proof that LC can carry antigens to lymphoid tissues. One study in sheep showed that VC isolated from pseudo-afferent lymph carried proteins injected intradermally, as assessed by antigen-specific proliferation assays [96] but, again, these cells could have taken up the antigens in the lymph rather than in the skin.

Direct evidence for uptake of antigen and intracellular processing by LC is scarce 
and, often, circumstantial. Puré et al [35] have shown that antigen-processing by LC is sensitive to chloroquine and that LC endocytose rhodamine-ovalbumin into perinuclear granules. Interestingly, both cultured and fresh LC internalised equivalent amounts of antigen, as assayed by fluorescence microscopy, the only study where the relative capacities of mature and immature LC to internalise antigen have been compared. Endocytosis in situ by LC has been reported for peroxidase injected intradermally in humans [164] or administered intravaginally in mice [165] but no evidence for uptake of 125I-flagellin or chromium was found in similar experiments on guinea pigs $[166,167]$. Uptake of viruses by sheep VC has been reported [94] but it is unclear whether it represents a physiological process or a viral mechanism leading to infection. $\mathrm{VC}$ from mice only internalise small amounts of various proteins in vitro [91] and internalisation of ferritin by rat VC in vitro is not detectable by electron microscopy [89]. VC from rabbits "did not phagocytose carbon particles, sheep erythrocytes, ferritin, PTAP diphtheria toxoid or ${ }^{125} I-K L H^{\prime \prime}[87]$.

The question of Fc receptor-mediated uptake of immune complexes by DC is a vexed one. VC from sheep do not endocytose immune complexes in vitro although they may do so in vivo [93]. One report of Fc receptor-mediated uptake by sheep VC in vitro and in vivo using FACS analysis fails to differentiate between true internalisation and binding to the cell surface since it is claimed that "the uptake mechanism ... was temperature independent" in experiments at $4^{\circ} \mathrm{C}, 25^{\circ} \mathrm{C}$ and $37^{\circ} \mathrm{C}$ [95]. Silberberg-Sinakin and colleagues [168] found that, following injection of immune complexes into the skin of guinea pigs, BG-containing cells in the $\mathrm{LN}$ showed signs of damage which were absent in complement-deficient animals. In neither animal was there substantial uptake of the complexes although DC could bind the complexes; damage likely occurred because binding of complexes to the DC surface in normal guinea pigs led to activation of complement via the classical pathway. Thus, so far, reports of immune complex uptake by LC remain largely unconvincing although binding via (internalisation incompetent?) Fc $\gamma$ receptors certainly occurs. However, uptake via FcE receptors is likely to play a role in 
atopic disease. Human LC have recently been shown to express the high affinity IgE receptor, FceRI [169], and can be induced to express FceRII (CD23) by IL -4 and/or IFN- $\gamma$ [170]. T cells primed against house dust mite allergen are found only in atopic dermatitis patients whose epidermal LC have cell surface-associated IgE and presentation of the allergen by these LC in vitro can be inhibited by treatment with anti-IgE [171]. T cell restimulation by IgE-bearing LC that have captured allergen in the airways is likely to be involved in maintaining high levels of serum IgE due to T cell help in antibody formation.

In the light of such conflicting reports on the endocytic capacity of DC it is interesting to note that the unique structural markers of LC in many species, Birbeck granules, have an endocytic origin $[172,173]$. In addition, BG are accessible to surface MHC molecules cross-linked by antibodies $[174,175]$ and may recycle antigens back to the cell membrane [176]. At present the function of BG remains unknown but these observations could have important implications for antigen processing and presentation.

\section{Phagocytosis of antigens by DC}

Phagocytosis of particulate antigens is a process fundamentally distinct from the uptake of soluble substances discussed above. Most cells are capable of endocytosing solutes from the external milieu but uptake of particles is believed to be restricted to specialised cells, often called "professional" phagocytes. Studies on the phenotype of DC have emphasised their non-phagocytic nature $[55,59,65,67,71,78,89,153,177]$ without addressing the paradox that a cell that initiates immune responses to exogenous antigen must be able to take up particulate, often pathogenic, organisms. A limited number of reports that are available provide contradictory results on the capacity of DC to present bacterial and other particulate antigens. Human blood DC certainly are very potent at presenting soluble bacterial superantigens, DC:T cell ratios of 1:300 being able to induce responses to femtomolar concentrations of Staphyloccocal enterotoxin E [178]. Two groups found that $\mathrm{DC}$ were potent accessory cells in anti-mycobacterial $\mathrm{T}$ cell responses to intact $M$. tuberculosis $[154,179]$ but, in contrast, it has been claimed that DC need to co- 
operate with $\mathrm{M} \varnothing$ in responses to intact Corynebacterium parvum [180]. However, the DC in the latter report were isolated by unconventional methods and were poor stimulators of the MLR, throwing doubt on the validity of the results. Controversial data have been obtained in experiments where soluble antigens were conjugated to latex beads and tested for presentation to T cell clones by DC: Kapsenberg et al [155] found that insolubilised ovalbumin could no longer be presented by DC whereas another group reported that hen egg lysozyme coupled to latex beads was presented [144]. Heterogeneity between T cell clones may be at the root of such discrepancies. Katz and colleagues [181] found that some murine $\mathrm{T}$ helper clones were more responsive to presentation by $\mathrm{DC}$ than by $\mathrm{M} \varnothing$ whereas the converse was true for other clones. Interestingly, there was no evidence that clones against a particulate antigen (horse erythrocytes) required $\mathrm{M} \varnothing$ as accessory cells and clones against soluble antigens (ovalbumin) responded better to DC: some clones against horse red blood cells were stimulated better by DC showing that these cells could present particulate antigen [181].

Thus, it seems that DC have the capacity to present particulate antigens, at least in some cases. Despite contradictory results on presentation, all reports agree on the fact that the DC were largely non-phagocytic for the antigens tested $[144,154,155,179-181]$. These findings have led to the suggestion that DC might have the ability to process particulate antigens extracellularly by means of ectoenzymes [144, 154]. However, with rare exceptions [154], these reports did not address the possibility that a small amount of soluble antigen was present in the preparations of insoluble antigens that were fed to the cells and was responsible for the activity observed. Furthermore, the data do not preclude the possibility that a limited extent of phagocytosis by a minority of DC occurred. A common feature of all these reports was the use of mature DC (overnight-cultured splenic DC from mice, human blood DC). It is, therefore, possible that processing of particulate antigens is primarily a function of immature $\mathrm{DC}$, similarly to processing of soluble antigens, and that mature DC may contain (or not, depending on the experimental procedure) some of these cells, explaining the activity reported by some workers (see 
chapter 6).

Reports of phagocytosis by immature DC are scarce and, largely, inferential; many fail to distinguish between endocytosis and phagocytosis. Veiled cells from rat contain Feulgen-positive inclusions (i.e., DNA-containing) reminiscent of a phagocytic past but do not phagocytose particles in vitro [89]. Bacterial antigens have also been described associated with "a very small minority" of veiled cells in lymph draining the gut of Salmonella-infected rats [182]. Some of these cells contained recognisable bacteria while others contained only debris. Some VC from sheep phagocytose latex beads but do not take up opsonised red blood cells or bacteria in culture [94]. The ability of Langerhans cells to phagocytose antigens has not been studied in detail. Poulter et al [183] described rare $\mathrm{CD} 1 \mathrm{a}^{+}$cells in the dermis of leprous skin which contained intracellular Mycobacterium leprae, but no such cells were found in the epidermis. LC with intracellular "treponeme-like material" may be present in skin during granulomatous syphilis [184]. In a review, Schuler and colleagues [185] claim that guinea pig LC coated with anti-Ia antibodies can phagocytose Staphylococcus aureus used for panning the cells but these data have not been published. We are only aware of one in vitro study where $\mathrm{FCR}^{+}$cells isolated from murine epidermis (taken to be the equivalent to LC but without Birbeck granules) were said to have some phagocytic capacity for $1 \mu \mathrm{m}$ latex beads and IgG-coated sheep erythrocytes [186]. ${ }^{6}$

Other reports of phagocytosis by DC are difficult to conciliate with an immunological role and, instead, suggest DC can play a scavenger part in homeostasis. LC phagocytose apoptotic epithelia cells in vaginal epithelium [187] and some human LC have ingested cell debris after culture [185]. VC in the lymph of sheep sometimes show vacuoles containing erythrocytes [93]. Interestingly, interdigitating cells in LN ingest allogeneic lymphocytes injected intravenously or into the footpads of nude rats but only after they have been killed by natural killer cells [188].

${ }^{6}$ In the course of this study we have become aware of efforts by another group to define a possible role for murine LC in infection by Leishmania major. That work, part of which is still in press, is reviewed in the discussion (chapter 6). 
Clearly, it is necessary to define the ability of different DC to take up and process particulate (and soluble) antigens. Changes in endo/phagocytic ability during DC maturation could help resolve the controversies apparent in the literature but have never been investigated in a systematic fashion. As summed up by G. G. MacPherson in the introduction to a recent forum on dendritic cells, "Whether DC have a "window" in their life history when they are capable of endocytosing particulate antigens remains to be determined." [112]. A detailed study of the ability of DC to phagocytose particulate antigens and to localise such a "window" is the objective of this thesis.

\section{3 - Phagocytosis}

Soluble antigens and small particles are internalised by endocytosis. Phagocytosis, on the other hand, refers to the engulfment of large particulate material (see below). Mononuclear and polymorphonuclear leukocytes are the main phagocytes involved in the clearance of particulate antigens (yeasts, bacteria, tumour cells) and have been termed "professional phagocytes" for this reason. Their importance is dramatically illustrated in patients who suffer from defects in phagocyte function. For example, in patients with the leukocyte adhesion syndrome a genetic defect in $\beta_{2}$-integrins prevents recruitment of phagocytes from the blood into extravascular sites of inflammation and these patients suffer from recurrent bacterial and fungal infections. A monoclonal antibody against murine CR3 has the same effect in mice $[189,190]$. Although other cells are capable of phagocytosing particles in the mammalian host [191], we shall concentrate on "professional" phagocytes in the brief overview that follows.

\subsection{1 - The phagocytic pathway}

Phagocytosis and endocytosis are mechanistically different processes. Phagocytosis is totally abrogated by temperatures below $18^{\circ} \mathrm{C}$ whereas endocytosis still occurs below this temperature, albeit at reduced rates. In addition, unlike endocytosis, phagocytosis is totally dependent on polymerisation of actin filaments underlying the plasma membrane 
and can be selectively inhibited with agents that interfere with assembly of actin filaments such as cytochalasins. Microtubules are necessary for endocytosis and may also be necessary for phagocytosis via CR3 but not for phagocytosis mediated by FcR [192]. The size of the particle is another deciding factor, since particles internalised by endocytosis are normally smaller than $0.1-0.5 \mu \mathrm{m}$ in diameter $[25,191]$. Like any cut-off point, the size above which a particle is ingested by phagocytosis is relative. Ingestion of $1 \mu \mathrm{m}$ latex beads, for example, is thought to be a phagocytic process and, yet, unlike the ingestion of larger particles, it is only inefficiently inhibited by treatment with cytochalasin B [193]. However, it is fairly reasonable to say that particles larger than $0.5 \mu \mathrm{m}$ will require some phagocytic process for ingestion [191]. In endocytosis the plasma membrane forms an invagination that pinches off to become an endosome. Phagocytosis, on the other hand, involves projection of pseudopods that gradually englobe the target particle and fuse to generate a phagosome. The mechanism by which this process occurs is termed the "zipper" mechanism of phagocytosis and was elucidated in a series of elegant experiments by Griffin and colleagues [194, 195] (fig. 1.1). Particle recognition and initial attachment are dependent on receptor-ligand interactions and can occur at $4{ }^{\circ} \mathrm{C}$ [196]. Formation of pseudopods only occurs at $37^{\circ} \mathrm{C}$ and is dependent on the availability of further receptors on the phagocyte membrane free to interact with unligated ligand on the surface of the target (fig. 1.1, left hand side). If the availability is limited by removing ligands by proteolysis or blocking receptors surrounding the site of attachment, engulfment does not proceed and the particle remains bound [194]. A dramatic example is provided by the FcR-mediated ingestion of B lymphocytes opsonised with anti-immunoglobulin IgG [195]. When opsonisation is carried out at $4^{\circ} \mathrm{C}$ the lymphocytes are diffusely coated with IgG and, upon feeding to $M \varnothing$, ingestion ensues. When the opsonised lymphocytes are warmed up to $25^{\circ} \mathrm{C}$ prior to feeding to $M \varnothing$ to allow capping of the IgG-IgM membrane complexes attachment occurs through the cap but the cells are not ingested (fig. 1.1, right hand side). 


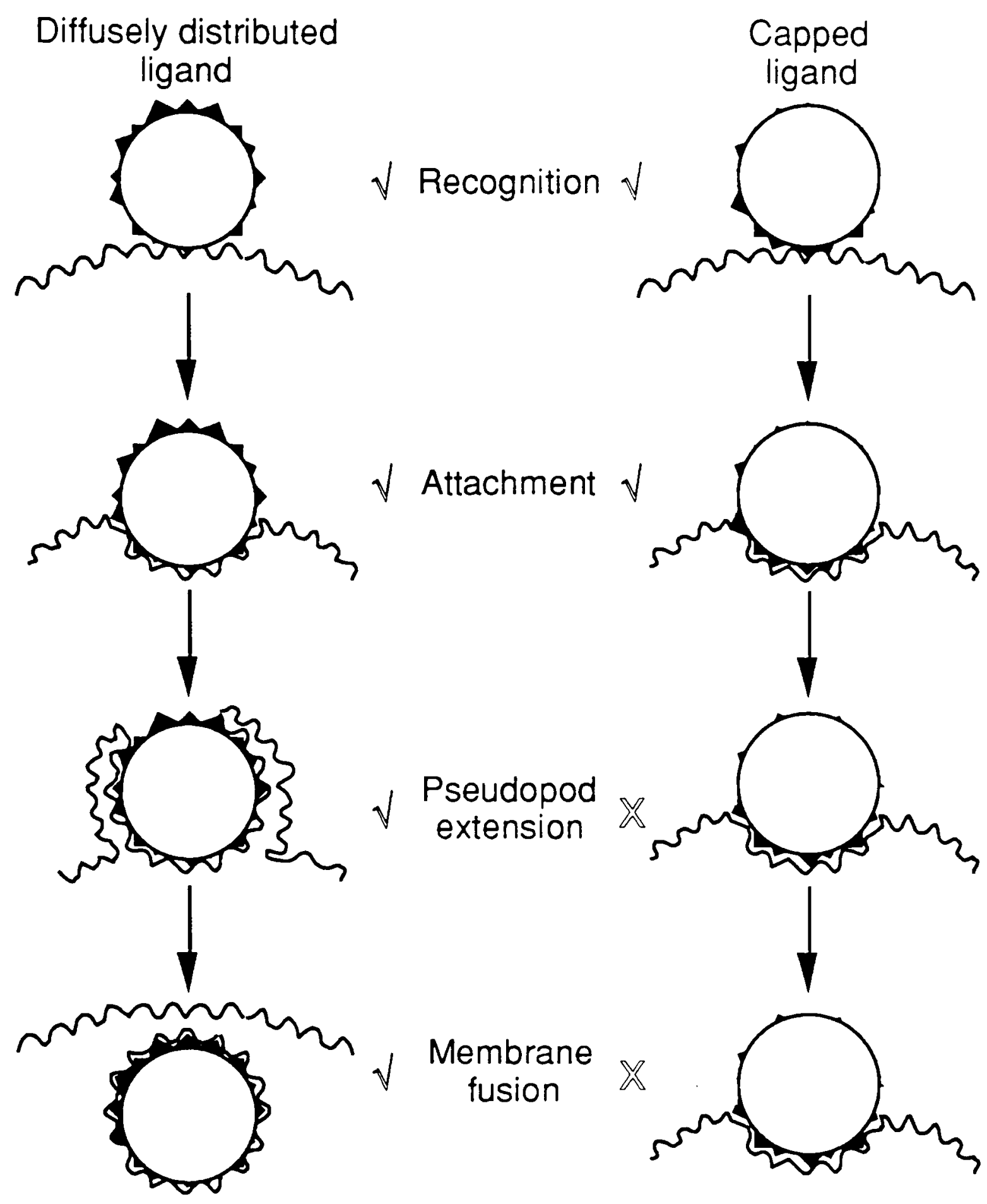

Fig. 1.1: Zipper mechanism of phagocytosis. Recognition and attachment of a target particle (filled) to phagocytic receptors on the phagocyte membrane promote circumferential spreading of the membrane around the particle provided ligand-receptor interactions are not spatially limiting. Not indicated are the actin filaments that underlie the membrane at sites of pseudopod formation. See text for details.

After the phagosome has formed it eventually fuses with pre-existing lysosomes to become a phagolysosome. MØ, however, can ingest $40 \%$ of their surface area in 15-30 min of phagocytosis [191]. Recycling of membrane from phagosomes before the fusion 
step is essential to ensure that the surface area of the phagocyte remains constant [30]. Recycling of phagosomal membrane back to the plasma membrane is extremely rapid and is essentially complete by $5-10 \mathrm{~min}$ at $37^{\circ} \mathrm{C}$ [197]. Such rapid recycling also contributes to the fact that the composition of plasma and phagosomal membranes is remarkably similar [197]. However, it is possible that during the actual process of phagosome formation some membrane proteins are sequestered preferentially in the advancing pseudopods. For example, the zipper model of phagocytosis would predict that the phagocytic receptors involved in particle recognition are selectively depleted from the membrane before recycling from phagosomes takes place. As expected, receptors which do not recycle and are degraded in the phagolysosomes after internalisation, such as Fc $\gamma \mathrm{R}$ in mouse $\mathrm{M} \emptyset$, are indeed depleted from the plasma membrane following phagocytosis [198].

Little is known about the intermediate steps between phagosome formation and fusion with lysosomes but, by analogy with the endocytic pathway, it is postulated that the early phagosome matures into a late phagosome before becoming a phagolysosome. The analogy is borne out by the fact that there is extensive communication between the phagocytic and endocytic pathway [30]. Newly-formed phagosomes start fusing with early endosomes before they fuse with late endosomes/lysosomes [199]. Degradation of internalised material also begins in the early phagosome, possibly due to the contribution of hydrolases from early endosomes $[199,200]$. These organelles may also bestow upon the phagosome the capacity to recycle membrane to the cell surface since phagosomederived transport vesicles containing partially degraded protein can fuse with early endosomes in vitro [200]. Alternatively, recycling from phagosomes may proceed directly to the cell surface. In contrast to these reports, Rabinowitz et al [26] have argued that fusion of phagosomes only occurs with components of the late endocytic pathway and have shown that the phagocytic and endocytic pathways merge at the level of their terminal compartments to form phagolysosomes/late endosomes. A recent report makes clever use of parasitophorous vacuoles in Leishmania amazonensis-infected $\mathrm{M} \varnothing$ as a model to study fusion of new phagosomes [201]. Such approaches will contribute to our understanding of 
membrane dynamics during phagocytosis although there is the danger that they represent a process altered during pathological conditions.

\subsection{2 - Receptors involved in phagocytosis}

As already implied above (see fig. 1.1), phagocytosis is dependent on the presence of specific phagocytic receptors. It is believed that even, so-called, "non-specific" phagocytosis of latex beads or aldehyde-fixed erythrocytes is mediated by receptors yet to be identified [191]. For example, the ingestion of zymosan, once thought to be a marker for non-specific phagocytosis, is in fact due to specific receptors (see section 5.1). Receptors may be constitutively active (e.g. Fc $\gamma$ receptors, mannose receptor) or require some form of activation or a co-stimulatory signal to become phagocytosis-competent (e.g. CR3). The importance of receptors in phagocytosis has been strengthened recently with the observations that transfection of $M \varnothing$ mannose receptor [202, 203] into non-phagocytic cells confers the ability to phagocytose the respective ligands. If confirmed with additional cell types and other receptors (Fc receptors: see below) this will be in marked contrast to earlier opinions that phagocytosis requires a specialised cell with a unique phagocytic "machinery". It is, of course, possible that "professional" phagocytes have additional mechanisms to increase the efficiency of the phagocytic process but the observation that a phagocytic receptor has the ability to mobilise the cytoskeleton of an heterologous cell where it is being expressed has important implications for the regulation of phagocytic activity and may help explain how "non-phagocytic" cells (e.g. keratinocytes [204]) can show signs of phagocytic activity.

The best studied phagocytic receptors are the Fc $\gamma$ and complement receptors of mouse macrophages [193]. Accordingly, their properties are briefly summarised below. The roles of other phagocytic receptors for polysaccharides are discussed in sections 5.1 and 5.3 in the context of phagocytosis of zymosan. 


\section{Fcy receptors}

Mouse macrophages have three Fc receptors for IgG encoded on three separate genes [119]. FcyRI (CD64) is a trypsin-sensitive receptor that binds monomeric IgG2a with moderate affinity. No monoclonal antibody is available against murine FçRI but a monoclonal (2.4G2) is available that recognises both Fc $\gamma \mathrm{RII}$ and FcyRIII. For this reason, for a long time, Fc $\gamma$ RIII was not recognised as a separate entity and most of the literature on Fc $\gamma$ RII fails to distinguish between the two receptors [119]. FcyRII (CDw32) and Fc $\gamma$ RIII (CD16), previously known as Fc $\gamma R I I \beta$ and Fc $\gamma R I I \alpha$, respectively, are encoded on separate genes but are $95 \%$ homologous and differ only in their cytoplasmic and transmembrane domains. Fc $\gamma$ RII is present on many different cell types and has low affinity for aggregated IgG1, IgG2b and IgG2b but not for monomeric IgG. It exists in two forms which have different cytoplasmic domains and are the product of two transcripts generated by alternative splicing of one exon. The b2 transcript is found exclusively on myeloid cells whereas the b1 transcript is expressed preferentially in lymphocytes [119]. Fc $\gamma R$ III expression in $M \varnothing$ is regulated and it is preferentially expressed over Fc $\gamma$ RII in thioglycollate-induced and $\gamma$-interferon $(\gamma$-IFN)-activated M $\varnothing$ [205].

$\mathrm{Fc} \gamma$ receptors in M $\varnothing$ are constitutively active and receptor cross-linking triggers a plethora of responses such as oxidative burst, increase in intracellular calcium, phagocytosis and cell-mediated cytotoxicity (reviewed in [206]). It is not clear which responses are mediated by which Fc $\gamma R$ because of the expression of multiple receptor types by most cells. It appears that all three Fc $\gamma \mathrm{R}$ are capable of mediating phagocytosis but expression of receptor cDNA in non-phagocytic cells suggests they need the presence of other receptors/phagocytic factors to exert their effect [191]. Activation of M $\varnothing$ cell lines with $\gamma$-IFN leads to increased expression of FcyRIII and concomitant increase in phagocytosis of IgG-opsonised sheep erythrocytes [205] and, yet, when the receptor is expressed in fibroblasts it mediates binding of IgG-coated erythrocytes but not ingestion [205]. Expression of murine Fc $\gamma$ RII in non-phagocytic cells does not allow ingestion of IgG-coated erythrocytes either [205] but enhances phagocytosis of heat-killed Toxoplasma 
gondii that have been opsonised with IgG [207]. Interestingly, both the b2 and bl transcripts of FcyRII could mediate phagocytosis of the opsonised parasite and internalisation was dependent on the integrity of the cytoplasmic tail [207]. It is thought that this effect is mediated by collaboration with another receptor that recognises the protozoon directly.

\section{Complement receptors}

Phagocytes possess three receptors capable of recognising derivatives of the third component of complement [208]. Complement receptor type 1 (CR1; CD35) and type 3 (CR3; CD11b/CD18) will bind sheep erythrocytes (SRBC) coated with C3b and C3bi, respectively. Like other $\beta_{2}$-integrins, CR3 is specific for the sequence arg-gly-asp (RGD) of $\mathrm{C} 3 \mathrm{bi}$ and may bind other proteins containing this motif such as fibrinogen or coagulation factor X [208]. Complement receptor type 4 (CR4; CD11c/CD18) is closely related to $\mathrm{CR} 3$ and also binds $\mathrm{C} 3$ bi. Unlike $\mathrm{Fc}$ receptors, $\mathrm{C} 3$ receptors are not constitutively active and are not capable of mediating internalisation by resident unstimulated M $\varnothing$ [191]. Activation of the cells with phorbol esters [209], T cell-derived cytokines or attachment to fibronectin- or serum amyloid P-coated surfaces [210] can induce $\mathrm{C} 3$ receptors to become phagocytosis-competent but activation is reversible and requires the continuous presence of the activating stimulus [211]. However, even in conditions where phagocytosis via $\mathrm{CR} 3$ or $\mathrm{CR} 1$ is readily promoted, cross-linking of these receptors does not induce secretion of reactive oxygen intermediates [212]. Binding via complement receptors is not restricted to $\mathrm{C} 3$-coated ligands. CR3 has been postulated to bind unopsonised zymosan particles (see section 5.1), Leishmania surface glycoprotein gp63 [213] and Bordetella pertussis [214]. Furthermore, inhibition of CR3 has been found to affect phagocytosis of particles recognised by other receptors such as IgG-coated erythrocytes and latex beads. The latter observations can be viewed in the context of a model for the role of CR3 in phagocytosis that is different from its role in ligand binding. Brown has proposed that $\mathrm{CR} 3$ and $\mathrm{CR} 4$ function to mobilise cytoskeletal elements during phagocytosis by other receptors and are required for multiple adhesive events without 
being involved in the ligand recognition process per se [215]. An immobile subset of CR3 has been shown to be necessary for phagocytosis of particles recognised by other receptors: the epitope involved is different from the binding site for $\mathrm{C} 3 \mathrm{bi}$ and antibodies against it block Fc receptor- and CR1-mediated phagocytosis and also block binding of target particles to CR1 without affecting rosetting of C3bi-coated erythrocytes [216]. This subset of immobile CR3 appears to be associated with actin filaments underlying the membrane since it can be rendered mobile by treatment with cytochalasins [216]. Presumably, immobile CR3 in unstimulated $\mathrm{M} \varnothing$ is not able to mobilise the cytoskeletal elements involved in phagocytosis, explaining why these cells can bind but not ingest C3bi-coated particles [215]. Following stimulation, immobile CR3 interacts with actin filaments and enhances binding to other receptor and promotes phagocytosis [215]. This may be why activated $\mathrm{M} \varnothing$ have greater phagocytic capacity in general, even for those particles that are internalised via constitutively active receptors.

\section{4 - Summary}

The purpose of this introduction has been to introduce a series of concepts that provide the theoretical framework for the experiments presented in the next chapters. We are interested in $\mathrm{T}$ cell responses to exogenous antigens (section 1.1) and we believe these are initiated by dendritic cells. However, mature DC have poor endocytic ability and lack the capacity to phagocytose particles (section 1.2) and, in that respect, are very different from other accessory cells such as macrophages. Undoubtedly, the capacity of $M \varnothing$ to ingest particulate antigens is at the root of their role as APC but this capacity should be viewed as having evolved, primarily, to destroy infectious pathogens rather than to elicit $\mathrm{T}$ cell responses (section 1.3). It is clear that DC are not typical phagocytes but, since very little antigen is required by $\mathrm{DC}$ to stimulate $\mathrm{T}$ cells, a small phagocytic capacity may be enough to generate immunogenic peptides. Given the consistent lack of phagocytic ability reported for mature DC and the hint that they have had a phagocytic past, we have examined the ability of immature DC, such as the Langerhans cell, to phagocytose antigens 
in vitro. We have found that these cells are phagocytic (chapter 3) but rapidly down regulate this capacity in culture (chapter 4$)$. We further characterised some of the receptors involved in the phagocytic process and followed their fate during maturation of LC in culture (chapter 5). Together, the results presented in the next chapters suggest that DC have the capacity to phagocytose antigens at defined stages of their life cycle and, most likely, this capacity allows them to participate in the generation of immune responses against particulate pathogens. 


\section{Chapter 2}

\section{Materials and Methods}

\section{1 - Experimental animals}

\section{2 - Reagents}

2.2.1 - Monoclonal antibodies

2.2.2 - Polyclonal antibodies

2.2.3 - Miscellaneous conjugates

2.2.4 - Phagocytic markers

2.2.5 - Endocytic markers and inhibitors

\section{3 - Cell preparations}

2.3.1 - Tissue culture reagents

2.3.2 - Cell counts

2.3.3 - Epidermal cells

2.3.4 - Langerhans cells

2.3.5 - Migratory Langerhans cells

2.3.6 - Splenic dendritic cells

2.3.7 - Peritoneal macrophages

2.3.8 - J774E cell line

2.4 - Immunofluorescent cell labelling

2.4.3 - Attachment of cells to PLL-coverslips

2.4.4 - Immunofluorescence labelling of attached cells

2.4.5 - Immunofluorescence labelling for FACS

2.5 - Phagocytic and endocytic flow cytometric assays

2.5.1 - Phagocytic assays on Langerhans cells

2.5.2 - Differentiating between bound and internalised particles

2.5.3 - Modification of the assay for splenic DC

2.5.4 - Modification of the assay for soluble tracers.

\section{6 - Electron microscopy}

2.6.1 - Transmission electron microscopy (Langerhans cells)

2.6.2 - Immuno-electron microscopy (splenic dendritic cells)

\section{8 - Protein biochemistry}

2.8.1 - Metabolic cell labelling

2.8.1 - Cell lysates

2.8.3 - Immunoprecipitation

2.8.4 - SDS-PAGE

2.8.5 - Western blotting

2.8.6 - Preparation of a $F\left(a b^{\prime}\right)_{2}$ fragment from anti-mouse mannose receptor antiserum 


\section{1 - Experimental animals}

Male $\mathrm{C} 57 \mathrm{Bl} / 6 \mathrm{~J}(\mathrm{H}-2 \mathrm{~b}), \mathrm{BALB} / \mathrm{c}(\mathrm{H}-2 \mathrm{~d})$ and $\mathrm{C} 3 \mathrm{H} / \mathrm{HeN}(\mathrm{H}-2 \mathrm{k})$ mice were purchased from Harlan OLAC Ltd (Bicester, U.K.) or from The Jackson Laboratory (Bar Harbor, ME, U.S.A.) for experiments performed at Washington University, St. Louis MO, U.S.A. Mice were used between 6-26 weeks of age.

\section{2 - Reagents}

\subsection{1 - Monoclonal antibodies}

The monoclonals used in this study are given in table 2.1 .

Table 2.1: Monoclonal antibodies against murine antigens used

\begin{tabular}{|c|c|c|c|c|c|}
\hline Antibody 1 & Host & Subclass & Antigen & Reference & Use \\
\hline $\mathrm{B} 21.2^{2}$ & Rat & $\mathrm{IgG} 2 \mathrm{~b}$ & $I-A^{b, d}$ & ATCC $^{3} ;$ TIB 229 & $\begin{array}{l}\text { marker for } \mathrm{C} 57 \mathrm{Bl} / 6 \\
\text { and BALB/c LC }\end{array}$ \\
\hline GK 1.5 & Rat & $\operatorname{IgG2b}$ & L3T4 (CD4) & ATCC; TIB 207 & $\begin{array}{l}\text { negative control for } \\
\text { B21.2 }\end{array}$ \\
\hline $10.2-16$ & Mouse & IgG2b & $I-A^{k, r, f, s}$ & ATCC; TIB 93 & $\begin{array}{l}\text { marker for } \\
\mathrm{C} 3 \mathrm{H} / \mathrm{HeN} \text { LC }\end{array}$ \\
\hline NLDC-145 & Rat & $\operatorname{IgG} 2 \mathrm{a}$ & $?$ & [70] & marker for $\mathrm{LC}$ \\
\hline N418 & Hamster & & $\begin{array}{l}\text { p150/95; (DC- } \\
\text { restricted) }\end{array}$ & ATCC; HB 224 & $\begin{array}{l}\text { marker for splenic } \\
\text { DC }\end{array}$ \\
\hline $\mathrm{M} 1 / 70^{4}$ & Rat & $\mathrm{IgG} 2 \mathrm{~b}$ & CR3 & ATCC; TIB 128 & $\begin{array}{l}\text { inhibition of } \\
\text { zymosan uptake }\end{array}$ \\
\hline $\mathrm{F} 4 / 80$ & Rat & $\operatorname{IgG} 2 b$ & $?$ & ATCC; HB 198 & $\begin{array}{l}\text { negative control for } \\
\mathrm{M} 1 / 70\end{array}$ \\
\hline $5 \mathrm{C} 6^{5}$ & Rat & $\operatorname{IgG} 2 b$ & $\overline{\mathrm{CR} 3}$ & [189] & $\begin{array}{l}\text { negative control for } \\
\mathrm{M} 1 / 70\end{array}$ \\
\hline $\mathrm{HO}-13-4$ & Rat & $\operatorname{IgM}$ & Thy-1.2 & ATCC; TIB 99 & $\begin{array}{l}\text { LC enrichment for } \\
\text { biochemical studies }\end{array}$ \\
\hline $\begin{array}{c}\text { M1/9.3.4. } \\
\text { HL. } 2\end{array}$ & Rat & $\operatorname{IgG} 2 \mathrm{a}$ & $\mathrm{CD} 45$ & ATCC; TIB 122 & $\begin{array}{l}\text { Panning of LC for } \\
\text { biochemical studies }\end{array}$ \\
\hline
\end{tabular}

1 Unless otherwise indicated all antibodies were used as culture supernatants.

2 Used as biotinylated purified IgG.

3 ATCC: American Tissue Culture Collection

4 Used as supernatant or as purified IgG (Serotec Ltd., Kidlington, Oxford, U.K.)

5 Used as purified IgG (gift from Prof. S. Gordon, Sir William Dunn School of

Pathology, Oxford) 


\subsection{2 - Polyclonal antibodies}

The polyclonals used for cell staining are summarised in table 2.2. Polyclonals used for other purposes are indicated in the relevant sections.

Table 2.2: Second stage antibodies used in cell staining

\begin{tabular}{ccccccc}
\hline Conjugate & Host & Against & Dilution & Supplier & Cat. no. & Comments \\
\hline FITC & Mouse & Rat & $1: 50$ & Jackson & $212-085-082$ & - \\
FITC & Donkey & Rabbit & $1: 100$ & Jackson & $711-096-132$ & $\mathrm{~F}\left(\mathrm{ab}^{\prime}\right)_{2}$ \\
Biotin & Donkey & Rat & $1: 50$ & Jackson & $712-065-138$ & - \\
Biotin & Sheep & Mouse & $1: 25$ & Sigma & B 8899 & F(ab') \\
PE & Goat & Rat & $1: 100$ & Seralab & SBA 3030-09 & - \\
- & Rabbit & Hamster & $1: 50$ & Sigma & H1643 & - \\
\hline
\end{tabular}

1 The optimal dilution of second stage antibody was determined by titration.

\subsection{3 - Miscellaneous conjugates}

Texas Red-streptavidin (TR-strp) was from Jackson Immunoresearch Laboratories, West Grove, PA, U.S.A., cat. no. 016-070-084, and was used at 1:1000 to 1:2000 dilution. Phycoerythrin-streptavidin (PE-strp) was from Southern Biotechnology Associates, Birmingham, AL, U.S.A., cat. no. 7100-09, and was used at 1:100 to 1:200 dilution.

\subsection{4 - Phagocytic markers}

Zymosan A from Saccharomyces cerevisiae was purchased from Sigma, Poole, U.K., and prepared by boiling for $30 \mathrm{~min}$ in cation-free phosphate buffered saline (PBS) followed by extensive washing in sterile PBS. Staphylococcus aureus and Corynebacterium parvum cultures, obtained from the Public Health Laboratory, John Radcliffe Hospital, Oxford, UK, were fixed in $37 \%$ formaldehyde after extensive washing in sterile PBS. Carboxylated fluorescent latex beads $(0.5,1$ and $3 \mu \mathrm{m}$ diameter $)$ were purchased from Polysciences Ltd, Warrington, PA, U.S.A. Sheep erythrocytes (SRBC) were obtained from Becton-Dickinson, Abingdon, UK, and opsonised with $\alpha-$ SRBC IgG (rabbit antiserum, diluted 1:200; Nordic Immunological Ltd, Maidenhead, U.K.) or with 
$\alpha-S R B C$ IgM (MAS 012b monoclonal antibody supernatant diluted 1:2; Sera-Lab Ltd, Crawley Down, U.K.) for $30 \mathrm{~min}$ at $37^{\circ} \mathrm{C}$. IgM-coated SRBC were further opsonised with fixed C3 essentially as described [217]. The optimal opsonisation protocol (determined by various titrations in pilot experiments) was as follows: freshly-thawed human $A B$ serum (Blood Transfusion Service, John Radcliffe Hospital, Oxford, U.K.; stored in aliquots at $-70^{\circ} \mathrm{C}$ ) was partially depleted of terminal complement components by incubating with zymosan for $30 \mathrm{~min}$ at $37^{\circ} \mathrm{C}(3 \mathrm{mg}$ zymosan $+150 \mu \mathrm{l}$ serum $+150 \mu \mathrm{l} \mathrm{PBS})$; zymosan was removed by centrifugation and the supernatant was added to the IgM-SRBC suspension (1:1) for $30 \mathrm{~min}$ at $37^{\circ} \mathrm{C}$.

Zymosan, bacteria and SRBC were conjugated to fluorescein isothiocyanate (FITC; Sigma) by incubating with fluorochrome overnight in $0.5 \mathrm{M} \mathrm{NaHCO}_{3}, \mathrm{pH} 9.5$, at either $4^{\circ} \mathrm{C}$ or room temperature with constant shaking. Conjugation was followed by extensive washing in sterile PBS and particles were irradiated with a ${ }^{137}$ cesium source before use. SRBC used for FITC conjugation were fixed for $30 \mathrm{~min} /$ ice with $3.7 \%$ formaldehyde in PBS. Unconjugated and FITC-conjugated zymosan and formalin-fixed bacteria were stored at $4{ }^{\circ} \mathrm{C}$ for up to three months without deterioration. Longer term storage was in aliquots at $-20^{\circ} \mathrm{C}$. Fixed SRBC and FITC-SRBC were prepared fresh for each experiment.

\subsection{5 - Endocytic markers and inhibitors}

FITC-conjugated bovine serum albumin (FITC-BSA), mannosylated FITC-BSA (FITC-ManBSA), mannan and glucan were purchased from Sigma. Two different yeast mannans (Sigma; cat. no. M3640 and M7504) and glucans from yeast (Sigma; cat no. G6513) and barley (Sigma; cat. no. G5011) were evaluated as inhibitors of zymosan uptake in pilot experiments and found to differ in their inhibitory capacity. Mannan, cat no. M7504, and yeast glucan, cat. no. G5011, were more potent inhibitors and were used consistently thenceforth. Yeast glucan was poorly soluble and only the soluble fraction (after incubation at $37^{\circ} \mathrm{C}$, overnight) was used. Highly purified soluble mannan and glucan and FITC-conjugated mannan were a gift from Dr. V. Kery, Washington University, St. 
Louis, MO, U.S.A.

\section{3 - Cell preparations}

\subsection{1 - Tissue culture reagents}

Media. Complete culture medium used for all primary cell cultures was RPMI-1640 (R0) supplemented with $10 \%$ heat inactivated $\left(20 \mathrm{~min} / 56^{\circ} \mathrm{C}\right)$ foetal bovine serum $(\mathrm{FCS})$ (both from Gibco BRL, Paisley, Scotland), 2mM glutamine (BDH Ltd., Warwicks, U.K.), 0.025mM 2-mercaptoethanol (Bio-Rad Laboratories Ltd, Hemel Hempstead, U.K.), $45 \mu \mathrm{g} / \mathrm{ml}$ penicillin, $45 \mu \mathrm{g} / \mathrm{ml}$ streptomycin and $90 \mu \mathrm{g} / \mathrm{ml}$ kanamycin (all antibiotics from Gibco BRL). Complete medium is abbreviated to R10. J774E cells were cultured in alphaMEM (Tissue Culture Supplies, Washington University School of Medicine, St. Louis, MO, U.S.A.) supplemented with 10\% FCS, 60mM thioguanine (Sigma, St. Louis, MO, U.S.A.) $45 \mu \mathrm{g} / \mathrm{ml}$ penicillin and $45 \mu \mathrm{g} / \mathrm{ml}$ streptomycin as described [218]. This culture medium is designated $\alpha-10$-thio.

Isotonic solutions. Cells were washed in sterile cation-free phosphate buffered saline (PBS). For mannose receptor binding assays PBS was supplemented with $0.9 \mathrm{mM}$ $\mathrm{Ca}^{2+}$ and $0.5 \mathrm{mM} \mathrm{Mg}^{2+}$. Sterile Hank's balanced salts solution without phenol red (HBSS), from Gibco BRL, was used in the preparation of epidermal cells.

Separation media. Dense BSA (refractive index 1.387) used in the enrichment of dendritic cells was prepared by dissolving 50g BSA powder (Sigma, fraction V, cat. no. A7906) in $32.5 \mathrm{ml}$ distilled water $+93.0 \mathrm{ml} \mathrm{PBS}$, overnight at $4^{\circ} \mathrm{C}$. The following day, the solution was allowed to reach room temperature and its $\mathrm{pH}$ was adjusted to 7.3-7.4 with $1 \mathrm{M} \mathrm{NaOH}$, keeping record of the volume added. The solution was diluted with $\mathrm{H}_{2} \mathrm{O}$ such that the combined volume of $\mathrm{H}_{2} \mathrm{O}$ and $\mathrm{NaOH}$ added was exactly $14.5 \mathrm{ml}$. Dense BSA was sterilised by filtering through a $0.45 \mu \mathrm{m}$ filter cartridge (Nalgene).

Tissue culture plastics. Unless otherwise indicated, all tissue culture plastic 
disposables were Falcon Labware and were purchased from Fahrenheit Lab Supplies, Milton Keynes, U.K. or from Tissue Culture Supplies, Washington University School of Medicine.

\section{$2.3 .2-$ Cell counts}

Viable cells were counted in $0.2 \%$ Trypan blue (Sigma) on an improved Neubauer hemocytometer. Total nucleated cell counts were performed in Turk's solution $(0.01 \%$ Gentian Violet in 3\% acetic acid).

\subsection{3 - Epidermal cells}

Epidermal cells (EC), containing 0.5-2\% Langerhans cells (LC), were prepared as in reference [120] with minor modifications. Ears from 15-60 mice were split under sterile conditions and dorsal and ventral halves were floated on HBSS in separate $100 \mathrm{~mm}$ Petri dishes. Trypsin and EDTA were added to $0.625 \%$ and $0.02 \%$ final concentration, respectively, and the dishes were incubated at $37^{\circ} \mathrm{C}$ for $30 \mathrm{~min}$ for the dorsal halves and $\mathrm{lh}$ for the ventral halves. Epidermal sheets were separated from dermis with the aid of forceps and were shaken on a sieve in HBSS containing 6.25\% FCS. The resulting cell suspension was filtered through nylon mesh, washed in R0 and plated at a density of $1-3 \times 10^{5}$ cells $/ \mathrm{cm}^{2}$ in 100 or $60 \mathrm{~mm}$ tissue culture dishes in $\mathrm{R} 10(10 \mathrm{ml}$ and $5 \mathrm{ml}$, respectively). This culture system promotes the maturation and viability of LC due to cytokines produced by viable keratinocytes [120]. The latter adhere rapidly to tissue culture plastic whereas LC remain in the non-adherent epidermal cell fraction and can be recovered at various culture times as a mixed population containing $1-4 \%$ LC. Time in culture is indicated in days or hours measured from the time of plating.

\subsection{4 - Langerhans cells}

Langerhans cells in epidermal cell cultures rapidly become low density as a result of maturation [120]. In some experiments requiring an enriched population of LC, non- 
adherent EC cultured for at least $12 \mathrm{~h}$ were fractionated over dense BSA. The cells were resuspended in $4 \mathrm{ml}$ dense BSA in $15 \mathrm{ml}$ tubes, overlayed with $2 \mathrm{ml} \mathrm{R} 10$ and centrifuged to equilibrium at $2000 \mathrm{~g}$ for $20 \mathrm{~min}\left(4^{\circ} \mathrm{C}\right)$. The low density "pellicle" contained $10-60 \% \mathrm{LC}$ depending on the time at which the cultures had been harvested and intrinsic variability between experiments. Phagocytosis of particles did not affect LC density and $<5 \%$ of LC were, routinely, in the high density fraction.

For some protein biochemistry experiments fresh LC were purified using immunomagnetic beads (Dynabeads, Dynal, Oslo, Norway) . $100 \mu$ l of sheep anti-rat Dynabeads $\mathrm{M}-450\left(4 \times 10^{8}\right.$ beads $\left./ \mathrm{ml}\right)$ were incubated with $2 \mathrm{ml} \mathrm{B} 21.2$ supernatant for $8 \mathrm{~h}$ with constant shaking, washed $3 \mathrm{x}$ in $\mathrm{R} 10$ and resuspended at $8 \times 10^{7}$ beads $/ \mathrm{ml}$. $8 \times 10^{6} \mathrm{EC}$ were extracted five times with $20 \mu \mathrm{l} \mathrm{B} 21.2-$ coated beads each time (4:1 bead to target cell ratio, overestimating the percentage of $\mathrm{LC}$ to $5 \%$ ). The five positively selected samples were pooled together, washed 2x in PBS and lysed in lysis buffer (see below).

\subsection{5 - Migratory Langerhans cells}

Migratory LC were prepared exactly as described [104]. Mouse ears were split and floated on $2 \mathrm{ml} \mathrm{R} 10$ in 24-well tissue culture trays, one half per well. Cultures were incubated for 2-6 days and $1 \mathrm{ml}$ of medium was replaced with fresh R10 daily after the first 2 days. Non-adherent migratory cells collected from the bottom of the wells contained around $60-70 \%$ LC as assessed by staining for MHC class II antigens.

\subsection{6 - Splenic dendritic cells}

Murine DC constitute a minor population of spleen cells and the traditional method for enrichment makes use of DC properties such as low density and transient adherence to tissue culture plastic [57]. Low density spleen cells (LOD) were prepared by fractionating splenocytes over dense BSA columns $\left(7 \times 10^{8}\right.$ nucleated cells/column). The fractionation procedure was exactly as for LC (see above). LOD were plated on tissue culture dishes in $\mathrm{R} 10\left(10^{8} / 100 \mathrm{~mm}\right.$ dish, $3 \times 10^{7} / 60 \mathrm{~mm}$ dish $)$ and incubated at $37^{\circ} \mathrm{C}$ for $1.5-2 \mathrm{~h}$ during which 
period DC and MØ adhere to the dish surface. Non-adherent cells were removed by gentle washing with warm R0 and low-density adherent cells (LODAC) were recultured in R10. Normally, LODAC were cultured overnight leading to detachment of the DC population which was recovered by washing the culture vessel with $\mathrm{R} 0$ [57]. Cells prepared in that way commonly contained $\geq 70 \% \mathrm{DC}$ and were not enriched further for the experiments described here. Recent reports, however, suggest the overnight culture period leads to phenotypic and functional changes in DC [44, 118, 126, 143]. For this reason, in some experiments, LODAC were harvested by vigorous washing with cold R0 4-6h after plating and were compared to LODAC that were incubated overnight. In yet another series of experiments, LOD were plated on bacteriologic plastic and DC were not enriched further.

\subsection{7 - Peritoneal macrophages}

Resident peritoneal exudate cells, used as a source of resident macrophages, were obtained by washing the peritoneal cavity of mice with $5 \mathrm{ml}$ ice-cold PBS. The peritoneal cells were plated on $13 \mathrm{~mm}$ acid-washed round coverslips in 24 -well plates at a density of $2-4 \times 10^{5}$ cells/well in $2 \mathrm{ml} \mathrm{R} 10$ or on Petri dishes in $5-10 \mathrm{ml} \mathrm{R} 10$ and were cultured for 2$6 \mathrm{~h}$ before washing non-adherent cells. Thioglycollate-induced peritoneal macrophages (thio-M $\varnothing$ ), were obtained in a similar manner from peritoneal exudate harvested $4 \mathrm{~d}$ after injection of $1 \mathrm{ml}$ thioglycollate broth (gift from Dr. R. da Silva, Sir William Dunn School of Pathology, Oxford). The resulting populations of adherent cells were $>90 \% \mathrm{M} \varnothing$ as assessed by morphological criteria and were used as positive controls in many experiments.

\subsection{8 - J774E cell line}

The mannose receptor-expressing mutant cell line, J774E [218], was used in developing assays for mannose receptor. The cell line was cultured in $\alpha-10$-thio in tissue culture flasks and passaged at confluence. Monolayers were detached by mechanical means to avoid the use of trypsin since mannose receptor is trypsin-sensitive. 


\section{4 - Immunofluorescent cell labelling}

\subsection{3 - Attachment of cells to PLL-coverslips}

Poly-L-lysine coated coverslips (PLL-coverslips) were prepared by incubating $13 \mathrm{~mm}$ acid-washed round coverslips in poly-L-lysine solution (Sigma; diluted as per manufacturer's instructions) for $30 \mathrm{~min}$ at room temperature. PLL-coverslips were washed and stored in distilled water. Before use, the coverslips were placed at the bottom of the wells in a $24-w e l l$ plate and allowed to dry in a sterile environment. Cells were attached to the glass surface by placing a $50 \mu 1$ droplet of cell suspension $\left(10^{5}-10^{6}\right.$ cells) on the coverslip without allowing liquid to overflow into the well. The droplets were incubated for $15-30 \mathrm{~min}$ at $37^{\circ} \mathrm{C}$ and cell attachment was monitored under an inverted microscope. Adherent cells were washed once in PBS at room temperature and fixed in $3.7 \%$ formaldehyde in PBS for $15 \mathrm{~min}$.

\subsection{4 - Immunofluorescence labelling of attached cells}

EC cultured with particles for the indicated times were fractionated over dense BSA and high and low density fractions were washed in R0 and plated on PLL-coverslips. After fixation, attached cells were stained for MHC class II antigens with biotinylatedB21.2 (Bt-B21.2; kindly prepared and made available by Mr. J. Roake in our laboratory) followed by PE-strp or TR-strp. Bt-B21.2 was omitted in control samples. Each incubation with antibody lasted 40-50 min and the antibodies were diluted in PBS containing $0.02 \%$ sodium azide and $1 \%$ heat-inactivated FCS (washing solution, WS). WS was also used to wash the coverslips between each stage. After staining, coverslips were rinsed in distilled water to remove PBS salts and were mounted in Aquamount mounting medium (BDH Ltd.) to help prevent fading of fluorescence. Stained cells were visualised and photographed on a Zeiss Axioskop microscope equipped with UV illumination and FITC and Texas Red fluorescence filters. 


\subsection{5 - Immunofluorescence labelling for FACS}

All staining for flow cytometric (FACS) analysis was carried out on cells fixed with $3.7 \%$ formaldehyde for $15 \mathrm{~min}$ on ice and washed in PBS; pilot experiments determined that the epitopes of interest were resistant to formaldehyde fixation. Fixed cells were placed in aliquots into LP3 tubes (Falcon Labware) that fitted the sample inlet of the flow cytometer and stained in these tubes at $5 \times 10^{5}-5 \times 10^{6}$ cells/tube. Incubations with antibodies (diluted in WS) were carried out on ice and lasted 40-60 min. All washes (two between each stage) were in WS and stained cells were stored in $1 \%$ formaldehyde in WS until analysis. Permeabilised cells for staining of intracellular antigens were prepared by a modification of a published procedure [219]. Cells fixed in $3.7 \%$ formaldehyde for 15 min on ice were washed once in PBS and permeabilised for 5 min on ice using $n-$ octyl- $\beta-D-$ glucopyranoside (Sigma) dissolved in WS at $8 \mathrm{mg} / \mathrm{ml}$. Permeabilised cells were washed twice in WS before staining.

In initial experiments, $\mathrm{LC}$ from $\mathrm{BALB} / \mathrm{c}$ or $\mathrm{C} 57 \mathrm{Bl} / 6$ mice in fixed $\mathrm{EC}$ suspensions were stained with B21.2 (neat supernatant) followed by PE-conjugated goat anti-rat or biotin-conjugated donkey anti-rat and PE-strp. GK1.5 was used as an isotype-matched negative control for B21.2 since murine LC do not express CD4. In subsequent experiments, LC were stained with Bt-B21.2 followed by PE-strp. Negative control samples were incubated in washing solution alone followed by PE-strp. LC from $\mathrm{C} 3 \mathrm{H} / \mathrm{HeN}$ mice were stained with $10.2-16$ (neat supernatant) or no primary antibody as a control, followed by biotin-conjugated sheep anti-mouse $F\left(a b^{\prime}\right)_{2}$ and PE-strp.

DC-enriched spleen cell suspensions (LOD or LODAC) were pre-blocked with heat-inactivated $\left(20 \mathrm{~min} / 56^{\circ} \mathrm{C}\right)$ normal mouse serum (Jackson Immunoresearch; diluted 1:25) and stained with neat supernatant of a hamster monoclonal antibody, N418, specific for splenic DC [75] (a gift from Dr. R.M. Steinman, Rockefeller University, NY, U.S.A.). This was followed by biotinylated goat anti-hamster $F\left(a b^{\prime}\right)_{2}$ and PE-strp. Omission of N418 served as the negative control since no irrelevant hamster antibody was available. 


\section{5 - Phagocytic and endocytic flow cytometric assays}

\subsection{1 - Phagocytic assays on Langerhans cells}

The flow cytometric assay for phagocytosis by LC is outlined in fig. 2.1. Bulk EC cultures were pulsed with fluorescent particles on tissue culture dishes for the indicated periods of time and the non-adherent cell fraction was harvested by washing the surface of the dish with cold R0. Cells were washed once in cold R0, fixed for $15 \mathrm{~min}$ on ice with 3.7\% formaldehyde in PBS, washed once in PBS and stored in WS until staining. For some phagocytosis inhibition studies, the non-adherent EC fraction was harvested before the assay and pre-incubated in polypropylene tubes (Falcon Labware) for $30 \mathrm{~min}$ with inhibitor before pulsing with particles in the same tubes in the constant presence of the inhibitor. Similar results were obtained with either tubes or culture dishes (not shown). LC stained for MHC class II antigens in conjunction with PE were analysed by flow cytometry on a FACScan (Becton Dickinson, Mountain View, CA, U.S.A.) using Consort 30 software. The instrument was calibrated each time using samples from the experiment itself. ${ }^{1}$ After gating out debris and free particles, live gates were set around the brightest $\mathrm{PE}^{+}$ population, avoiding Ialow contaminants. Up to $5000 \mathrm{LC}$ were acquired and analysed for FITC fluorescence. Two parameters were obtained from this analysis, the percentage of LC positive for FITC (\% uptake) and the mean FITC fluorescence of those cells. Although the data were collected using a logarithmic recorder, mean fluorescence values were converted to a linear scale. Results are often expressed as an index, the product of \% uptake and mean fluorescence. Examples of this analysis are illustrated in figs. 3.5 and 4.2 in the experimental chapters. Critical technical aspects of the method are discussed below.

\footnotetext{
${ }^{1}$ Experiments were planned so that there were always double negative and single positive samples available for calibrating the FACScan.
} 


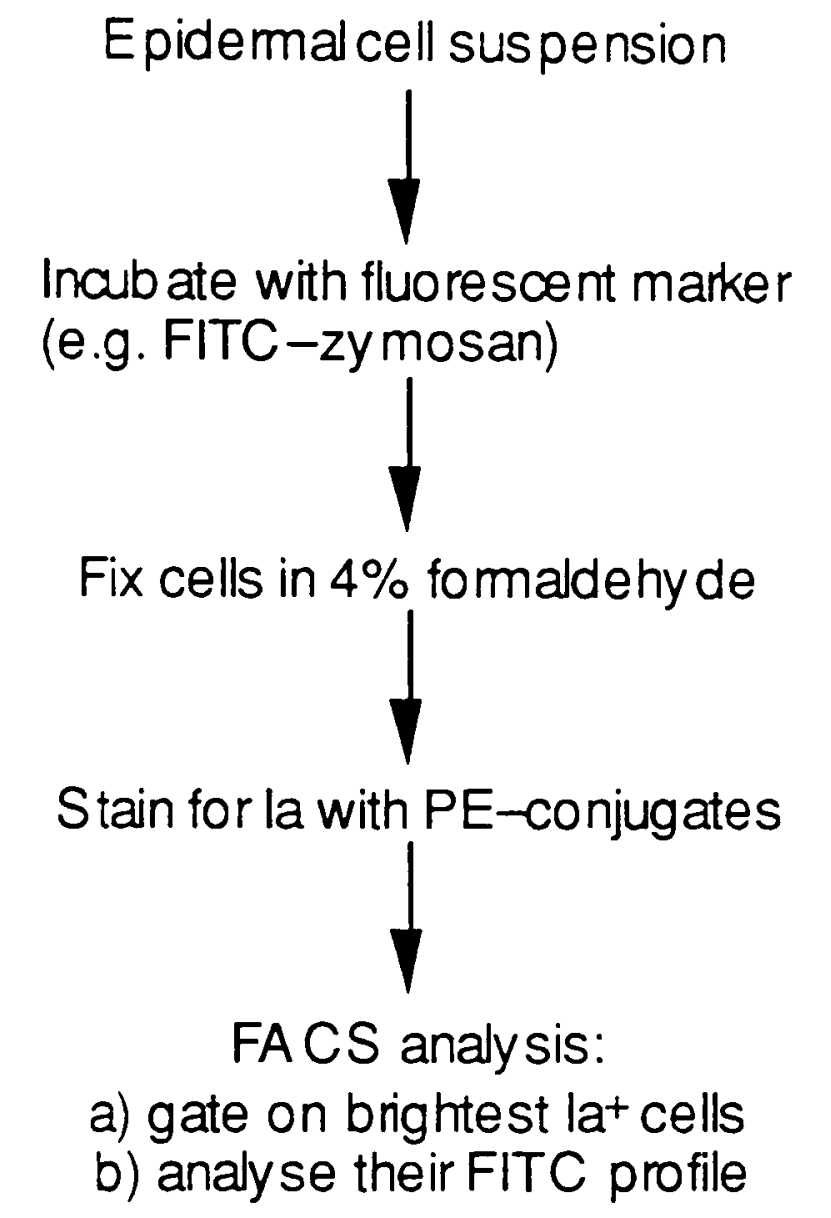

Fig. 2.1: Flow cytometric analysis of phagocytosis by LC.

One useful stratagem used in FACS analysis of Langerhans cells was to enrich for these cells artificially by scatter gating. The normal function of scatter gates is to separate cells from debris so that the fluorescence of the former is not affected by spurious events. Gating is a subjective procedure and an example is given in fig. $2.2 \mathrm{a}$ for bulk epidermal cells. EC have a broad scatter profile (fig. 2.2a) and the scatter gates are placed (bold rectangle) so as to exclude events with low scatter (bottom left corner) and those with very high forward and side scatter (top and right-hand margin). The former are likely to represent debris whereas the latter contain clumps of cells. When such broad scatter gates are utilised, the population of $\mathrm{Ia}^{+} \mathrm{LC}$ is small and not very distinct (fig. $2.2 \mathrm{c}$ ). The scatter profile of LC within the EC population can be determined by "backgating". On the basis of this knowledge, scatter gates can be made much narrower, thereby increasing the relative percentage of LC by excluding many keratinocytes (fig. 2.2b). This procedure was used routinely and resulted in clear $\mathrm{Ia}^{+}$populations (fig. $2.2 \mathrm{~d}$ ) that could subsequently be gated to exclude Ia- cells (see below). 
a

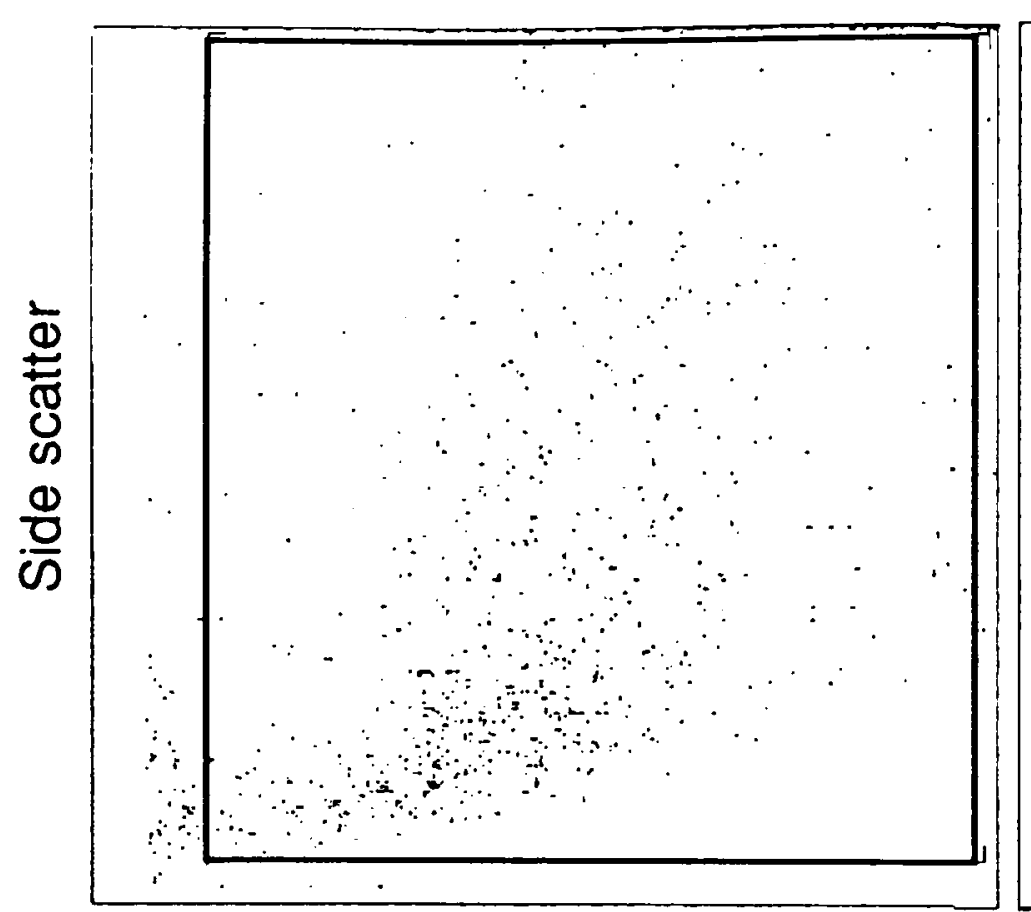

Forward scatter

C

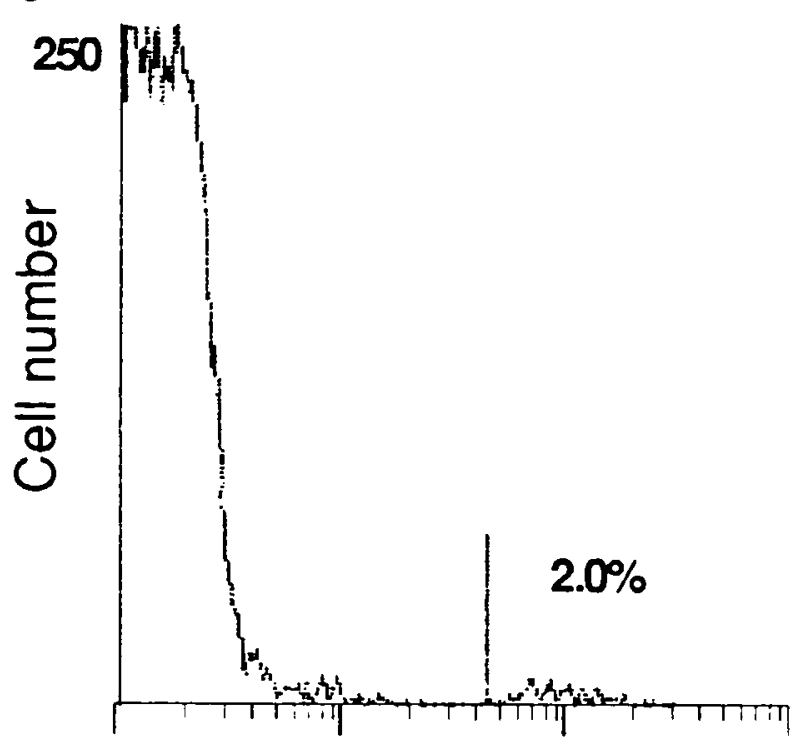

$\log _{10}$ B21.2 staining b

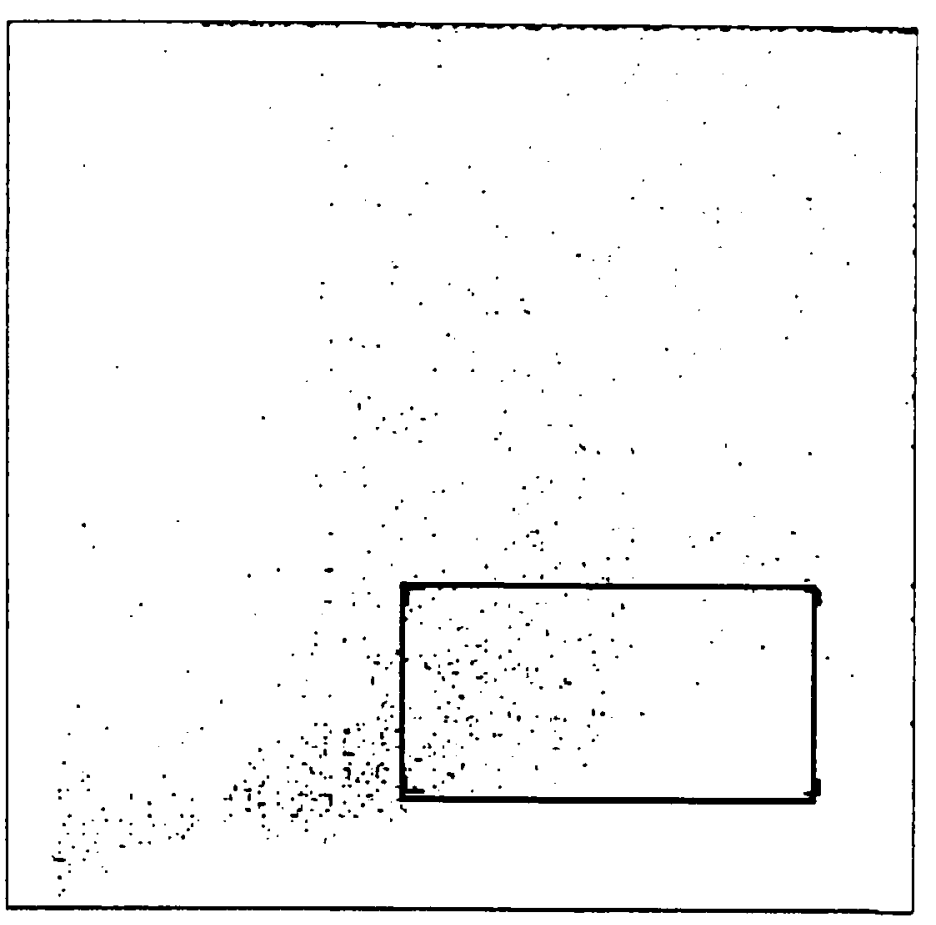

d

250

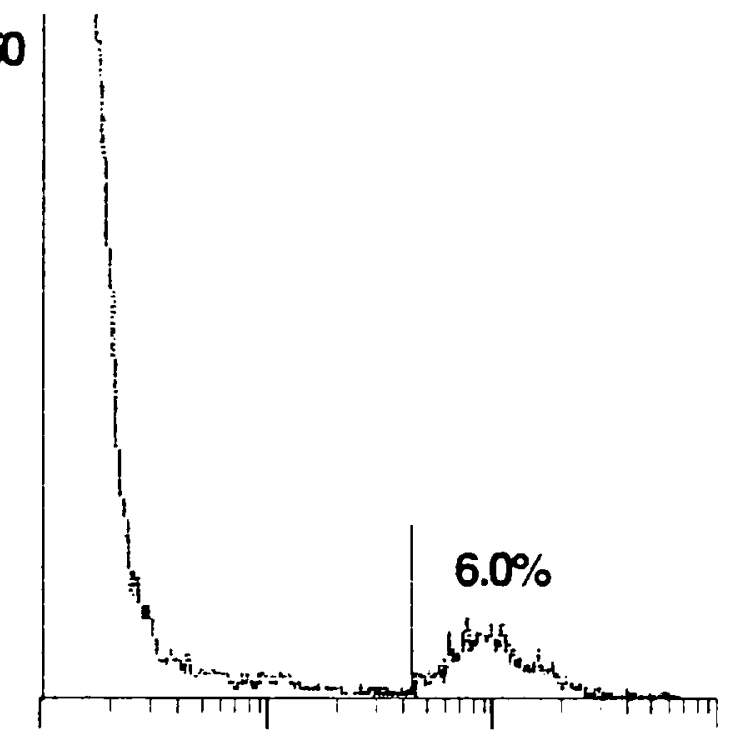

Fig. 2.2: Artificial enrichment of LC by scatter gating on bulk epidermal cell populations. a) wide scatter gates, b) Ia+_enriching scatter gates, c) PE fluorescence of gated cells from a), d) PE fluorescence of gated cells from b). Numbers indicate percentage of LC detected. See text for details. Bulk EC fixed after $72 \mathrm{~h}$ culture were stained with $\mathrm{Bt}-$ B21.2 followed by PE-strp.

The fluorescence gating procedure for LC is demonstrated in fig. 2.3 using Langerhans cells double labelled with anti-Ia (B21.2; PE channel) and NLDC-145 (FITC channel). Bulk epidermal cells, gated for scatter as above, contain a clear Ia ${ }^{+}, \mathrm{NLDC}_{-145^{+}}$ low-abundant population of Langerhans cells (fig. 2.3b; compare with the double negative 
control, fig. 2.3a). To analyse this population in more detail, the sample is run a second time while excluding Ia- cells by placing live gates on the PE channel. When the live gates are set on the basis of the negative control, the gated population contains NLDC- $145^{+}$, Iahigh $\mathrm{LC}$ but also Ialow contaminants that are clearly not LC since they do not express NLDC-145 (fig. 2.3c). On the other hand, if gates are set on the basis of the profile obtained for the positive sample, the resulting population contains only Iahigh, NLDC$145^{+}$cells and no contaminants (fig. $2.3 \mathrm{~d}$ ). Thus, although, in theory, gates should always be set according to a negative control, in the current series of experiments this approach proved to be counter-productive. This situation is thought to arise because a few keratinocytes bind Bt-B21.2 non-specifically (E. Kämpgen, personal communication), possibly via Fc receptors [220], and our negative control (omission of the primary antibody) does not account for this artefact. The appropriate control, a isotype-matched biotinylated irrelevant monoclonal, was not available. In spite of this shortcoming, labelling of LC with B21.2 was always so clear that live gates could safely be placed on the basis of the positive peak (fig. 2.3d). 


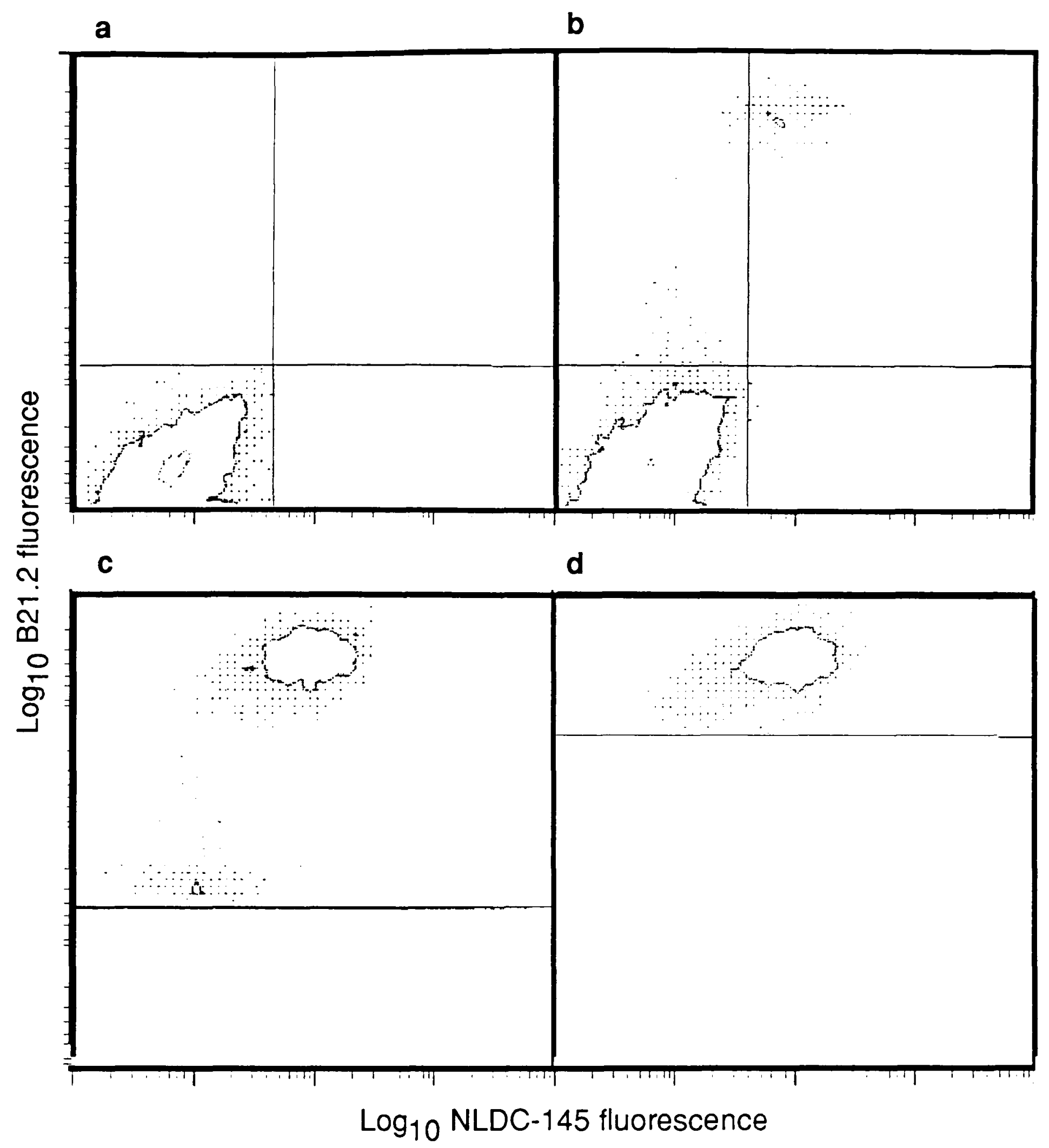

Fig. 2.3: Fluorescence gating procedure for $\mathbf{L C}$. a) negative control EC, b) positively stained EC, $\mathbf{c}) \mathrm{Ia}^{+}$cells gated according to negative control; bar marks position of gate, d) $\mathrm{Ia}^{+}$cells gated according to positive sample; bar marks position of gate. See text for details. EC fixed after overnight culture were reacted with NLDC-145 (neat supernatant) followed by FITC-conjugated mouse anti-rat. Free binding sites on the secondary antibody were blocked with heat-inactivated $\left(20 \mathrm{~min}\right.$ at $\left.56^{\circ} \mathrm{C}\right)$ normal rat serum $(1: 25)$ and cell-bound antibody was fixed in 1\% formaldehyde in PBS for $15 \mathrm{~min}$. After extensive washing the cells were stained with Bt-B21.2 followed by PE-strp. Omission of tho relevant primary antibodies served as controls for the specificity of staining and for crossreactivity. 
Fluorescence gates based on the positively stained cells were set at different levels for LC cultured for different times. This is due to the fact that LC in culture up regulate MHC class II antigens very rapidly with a consequent increase in PE fluorescence (fig. 2.4). As noted by others $[35,121,136]$, up regulation is very rapid, proceeds at its highest rate during the initial $12 \mathrm{~h}$ (fig. $2.4 \mathrm{~b}$ ) and is effectively complete by $24 \mathrm{~h}$ of culture (fig. 2.4a). The apparent decrease in fluorescence from $24 \mathrm{~h}$ to $72 \mathrm{~h}$ (fig. $2.4 \mathrm{a}$ ) is, possibly, due to an increase in the size of LC during maturation. An interesting feature is the apparent mouse strain difference in intensity of staining (fig. 2.4a). It is not clear whether this reflects a higher number of Ia antigens on the surface of BALB/c LC or higher affinity of the B21.2 antibody for I-Ad; the latter hypothesis is supported by data presented in the original publication describing the antibody [221].
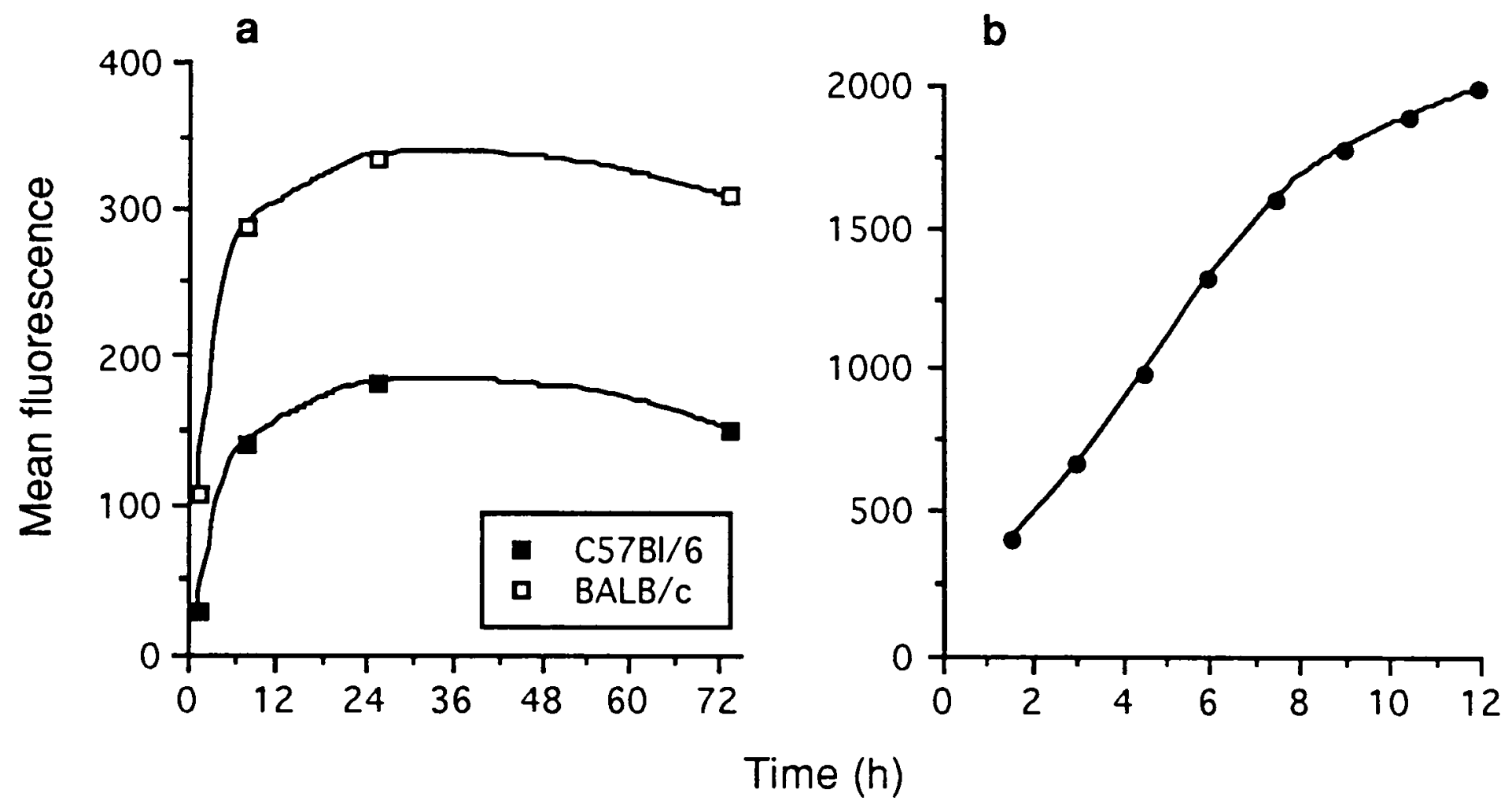

Fig. 2.4: Up regulation of $\mathrm{MHC}$ class II antigens during maturation of $\mathrm{LC}$ in culture. a) Ia expression by $\mathrm{LC}$ from $\mathrm{C} 57 \mathrm{Bl} / 6$ (closed symbols) or $\mathrm{BALB} / \mathrm{c}$ (open symbols) at different times in culture. b) up regulation of Ia during the first $12 \mathrm{~h}$ of culture $(\mathrm{C} 57 \mathrm{Bl} / 6)$. Data are mean PE fluorescence of gated LC that had been stained with Bt-B21.2 and PEstrp. a) and b) were obtained in separate experiments. 


\subsection{2 - Differentiating between bound and internalised particles}

A limitation of the flow cytometry phagocytic assay as described above is its inability to distinguish between particles bound to or only partly ingested by LC and those that are truly internalised. A number of flow cytometry assays for phagocytosis have been developed that obviate this problem by quenching the fluorescence of exposed particles with agents such as trypan blue or crystal violet prior to flow cytometry (reviewed in [222]) and we have successfully used trypan blue in some assays with macrophages (see table 5.5). Such an approach, however, could not be used for LC since these agents quench external fluorescence non-specifically and our assay depends on the fluorescence of labelled LC for cell identification. One way to obviate the problem was to attempt to quench the fluorescence of attached particles irreversibly before staining the cells for Ia. However, quenching by trypan blue was found to be reversible (fig. 2.5) such that this approach was not feasible.

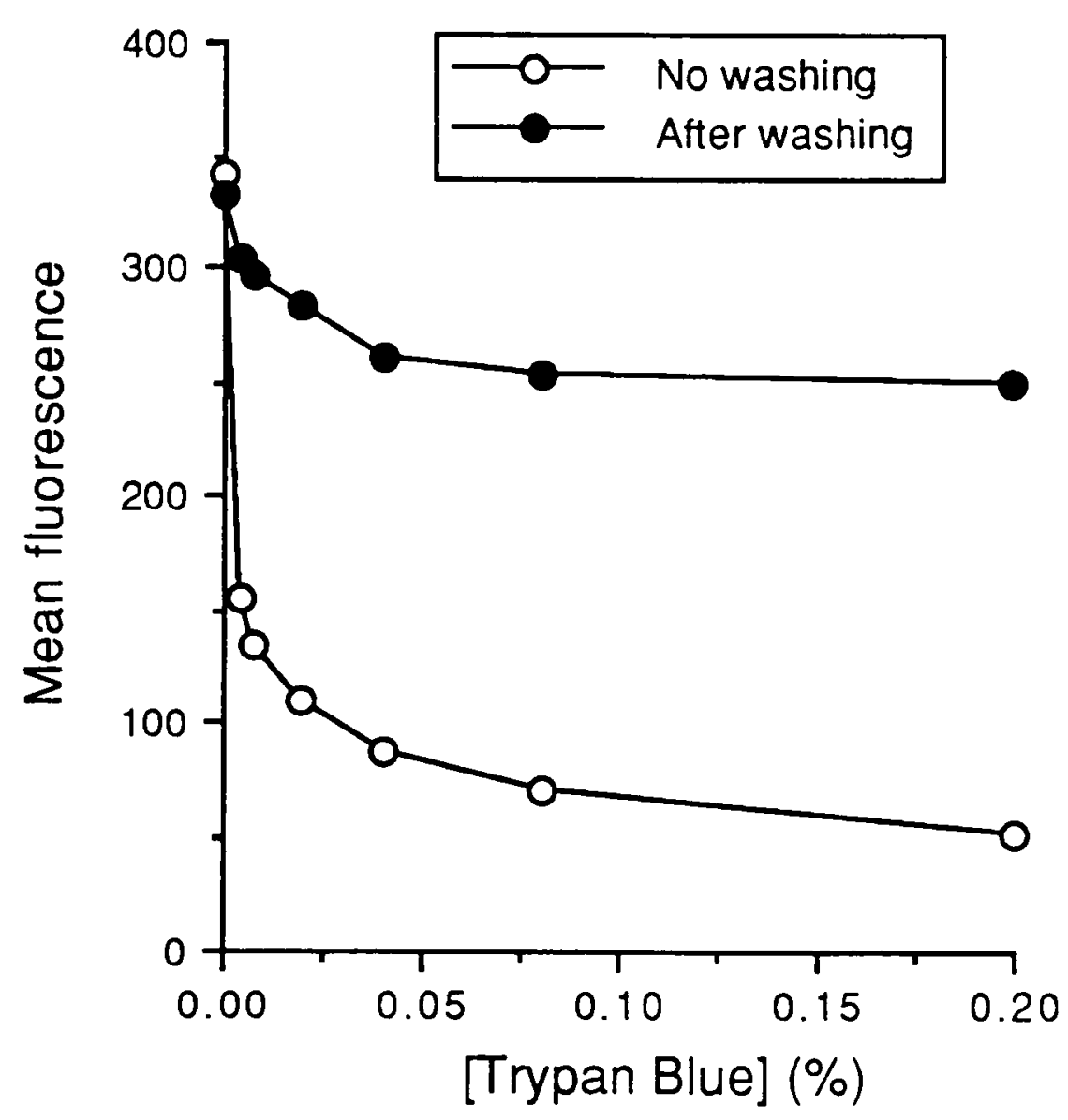

Fig. 2.5: Quenching of fluorescence by trypan blue is reversible. FITC-zymosan was incubated with the indicated concentration of trypan blue. Samples were analysed by FACS before (open circles) and after (closed circles) washing in WS. 
Attempts to remove adherent particles on LC by trypsinisation $[223,224]$ were also not successful (not shown) and bore the risk of destroying the Ia antigen necessary for identification of LC. Ultimately, differentiating bound and ingested particles proved not to be feasible in our assay. In practice, however, this caveat was of limited importance. Microscopical examination of LC in samples prepared for flow cytometry revealed that virtually all the LC associated with particles had internalised at least some of them. Thus, the percentage of LC associated with particles as determined by flow cytometry does correspond, in practice, to the percentage of LC that are phagocytic. Furthermore, the overall majority of LC-associated particles appeared to be truly ingested by phase contrast microscopy and this was confirmed by electron microscopy for zymosan and latex beads. One reason may be that loosely attached particles are lost during the vigorous vortexing employed to stain the cells. Further indication of the validity of the assay is given in table 2.3. When samples incubated at $37^{\circ} \mathrm{C}$ are compared with those incubated on ice, there are fewer LC with particles in the latter samples and those cells have a lower mean fluorescence indicating that they are associated with fewer particles per cell (table 2.3). This suggests that the signal obtained at $37^{\circ} \mathrm{C}$ is mostly due to particle ingestion.

Table 2.3: Attachment vs. ingestion of particles

\begin{tabular}{lcc}
\hline Incubation & $\begin{array}{c}\text { \% LC with } \\
\text { particles }\end{array}$ & $\begin{array}{c}\text { Mean } \\
\text { fluorescence }\end{array}$ \\
\hline $370^{\circ} \mathrm{C}$ & 85.3 & 969.31 \\
ice-cold & 37.2 & 653.08 \\
\hline LC were incubated with FITC-zymosan for 90 min \\
at the indicated temperature and analysed for \\
association with particles by FACS.
\end{tabular}

In view of these arguments, it was decided to use the assay without correcting for attached particles. However, this caveat is implicitly admitted in the use of the word "uptake" in relation to flow cytometry experiments throughout this thesis. 


\subsection{3 - Modification of the assay for splenic DC}

The FACS assay described above is not restricted to the analysis of Langerhans cells and was used successfully with other cell types. To analyse phagocytosis by splenic DC the cells were identified on the basis of the DC-restricted epitope of CD11c that is recognised by antibody N418 [75]. DC-enriched cell preparations (LOD or LODAC) were pulsed with particles, recovered and fixed exactly as for EC. DC were stained with N418 as described in section 2.4.5 and FACS analysis gating on N418-positive cells was carried out exactly as described for LC. Staining of DC with N418, however, was less clear than staining of LC for Ia (fig. 2.6; compare with fig. 2.2), particularly after overnight culture (fig. 2.6b). For this reason the gating procedure on $\mathrm{N} 18^{+}$cells was particularly stringent so as to include as few contaminants as possible, even at the risk of excluding a population of N418low DC.
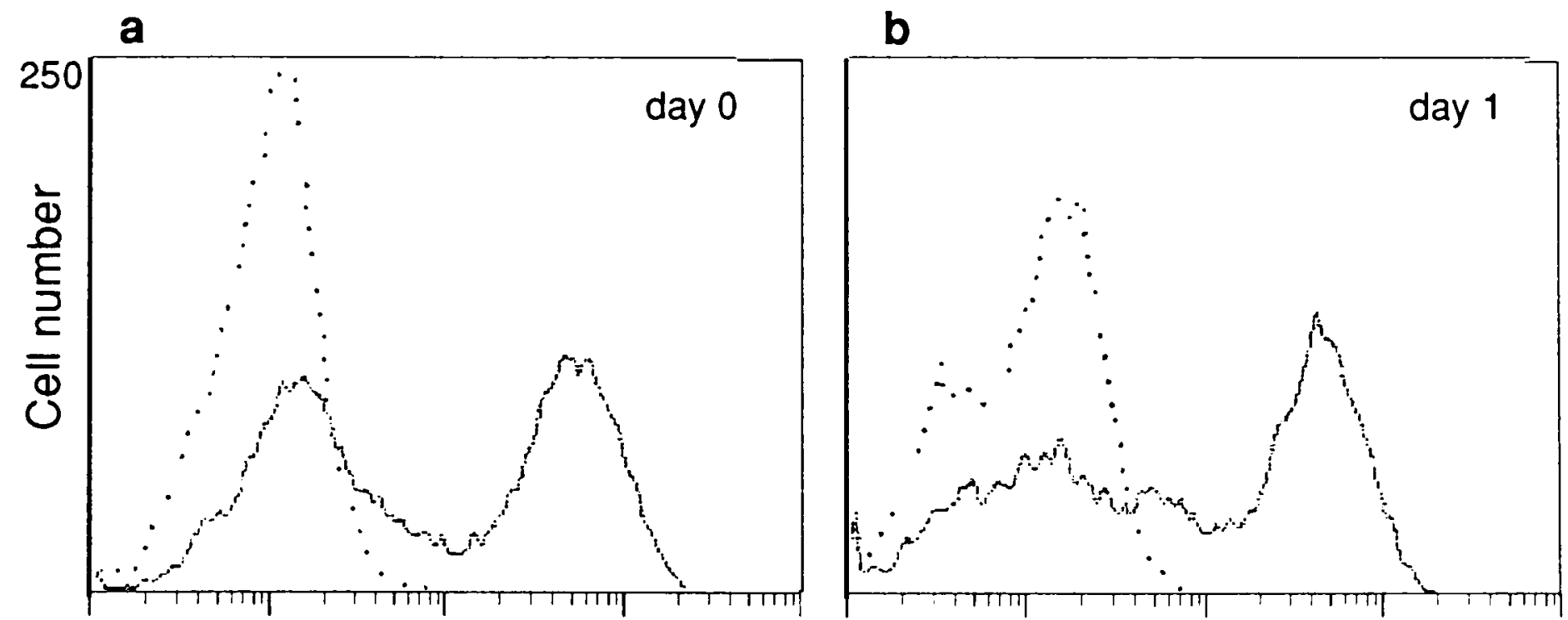

$\log _{10} \mathrm{~N} 418$ fluorescence

Fig. 2.6: $\mathbf{N 4 1 8}$ staining of splenic DC in enriched spleen populations. LODAC were recovered before (day $\mathbf{0}$; a) or after (day $\mathbf{1}$; b) overnight culture, fixed and stained for N418 (solid traces). Primary antibody was omitted from the control samples (dotted traces). Analysis of N418 expression was by single colour (PE) flow cytometry. 


\subsection{4-Modification of the assay for soluble tracers.}

A simple modification of the assay allowed the study of uptake by LC of soluble tracers instead of particles. For this approach, non-adherent EC were often harvested at various culture times before the assay and incubated with tracer in polypropylene tubes as in the phagocytosis experiments with inhibitors (see above). The soluble tracers included non-specific pinocytic tracers (FITC-BSA and Lucifer Yellow) and ligands for receptormediated endocytosis (FITC-ManBSA). After the assay cells were washed in cold R0 and fixed as described. Samples were stored in WS until staining for MHC class II antigens and FACS analysis was performed exactly as for phagocytic assays.

\section{6 - Electron microscopy}

\subsection{1 - Transmission electron microscopy (Langerhans cells)}

EC suspensions, pulsed with particles overnight, were fractionated over dense BSA and the low density fraction was washed and fixed in $2.5 \%$ glutaraldehyde in $0.1 \mathrm{M}$ sodium cacodylate/1\% sucrose buffer (60 min total). After extensive washing in the same buffer, pellets were post-fixed in $2 \%$ osmium tetroxide $(50 \mathrm{~min}$ ) and embedded in $1 \%$ agar to facilitate handling. Agar fragments were dehydrated through a graded ethanol series and embedded in Emix resin (Agar Scientific, Stansted, U.K.) using propylene oxide as the linking agent. After polymerisation, thin sections were cut on a Reichert-Jung ultramicrotome, stained with lead citrate and examined and photographed in a JEOL $100 \mathrm{CX}$ electron microscope operating at $80 \mathrm{kV}$. The author is grateful to Prof. S. Gordon and to Mrs. M. Bergin-Cartwright for use of the electron microscopy facilities at the Sir William Dunn School of Pathology, Oxford. Other samples were kindly processed by Dr. D. Ferguson, John Radcliffe Hospital, Oxford, U.K., using a similar protocol to the one above except that dehydration was carried out using an acetone series eliminating the need for linking agent. 


\subsection{2 - Immuno-electron microscopy (splenic dendritic cells)}

Low density adherent cells, pulsed with particles for 6 hours, were processed for immunoelectron microscopy (immuno-EM) exactly as described [225]. Cryosections were cut at $-90^{\circ} \mathrm{C}$ with a Reichert-Jung Ultra Cut ultramicrotome equipped with a FC4E cryosystem (Leica Inc., Deerfield, IL, U.S.A.) and were stained with N418 followed by rabbit anti-hamster and a protein A-10nm gold conjugate prepared according to Slot \& Geuze, 1985 [226]. Staining was carried out as in reference [225] except that PBS containing $10 \%$ FCS was substituted for $0.1 \%$ BSA-PBS. Specimens were viewed and photographed in a Zeiss E.M. 10A electron microscope. Samples for immuno-EM were kindly processed by Mrs. M.A. Levy, Dept. of Cell Biology and Physiology, Washington University, U.S.A., after the initial fixation step by the author.

\section{8 - Protein biochemistry}

The protein biochemistry methods described below were used to characterise a new rabbit antiserum raised against purified murine mannose receptor in Prof. P.D. Stahl's laboratory (Washington University, St. Louis, MO, U.S.A.), and to evaluate mannose receptor expression by LC using this reagent.

\subsection{1 - Metabolic cell labelling}

$\mathrm{M} \varnothing$ plated on 6-well tissue culture trays were starved of cysteine and methionine by incubating for $1 \mathrm{~h}$ at $37^{\circ} \mathrm{C}$ in starve medium (cysteine/methionine-free alpha-MEM, Tissue Culture Supplies, Washington University School of Medicine, supplemented with antibiotics and $10 \%$ FCS, dialysed to remove cys/met). Cells were then pulsed with $0.25 \mathrm{mCi} / \mathrm{ml}^{35} \mathrm{~S}$-labelled cys/met (Tran ${ }^{35} \mathrm{~S}$-Label; ICN Biomedicals, Irvine, CA, U.S.A.) in starve medium for $1 \mathrm{~h}$ at $37^{\circ} \mathrm{C}$. After labelling, cultures were washed three times with alpha-MEM and chased for $10 \mathrm{~min}$ in alpha-MEM containing a $10 \mathrm{x}$ excess of unlabelled cys/met. 


\subsection{1-Cell lysates}

Cells were washed in PBS and lysed either directly on tissue culture vessels (MØ) or in microfuge tubes (EC, LC) at $\approx 10^{7}$ cells $/ \mathrm{ml}$. Lysis buffer was $0.5 \%$ Triton $X-100$ in PBS containing $0.02 \%$ phenylmethylsulfonyl fluoride (PMSF; Sigma) and lysis was allowed to proceed for $15 \mathrm{~min}$ on ice. Lysates for immunoprecipitation were processed straightaway. Lysates for Western blotting were microfuged for $10 \mathrm{~min}$ to remove nuclei and cell debris and the supernatants were frozen until electrophoresis.

Protein concentrations in lysates were determined in triplicate on microtitre plates using the Bio-Rad Protein Assay (Bio-Rad, Richmond, CA, U.S.A.) or the Bio-Rad DC Protein Assay (Bio-Rad, Hemel Hempstead, U.K.) as per manufacturer's instructions. BSA or bovine IgG were used as standards.

\subsection{3 - Immunoprecipitation}

Lysates from metabolically-labelled $M \varnothing$ were pre-cleared with protein Asepharose (Prot A-seph; Sigma) for $30 \mathrm{~min}$ at $4^{\circ} \mathrm{C}$. Prot $\mathrm{A}-\mathrm{seph}$ and cell debris were removed by centrifugation for $10 \mathrm{~min}$ and the protein concentration of the supernatant was determined. $14 \mu \mathrm{g}$ of protein in a total volume of $500 \mu \mathrm{l}$ were reacted with a rabbit antiserum against murine mannose receptor (1:500) or with normal rabbit serum as a negative control for $2 \mathrm{~h}$ at $4^{\circ} \mathrm{C}$ with constant rocking. Immune complexes were collected on Prot $\mathrm{A}$-seph $(50 \mu \mathrm{l})$ for $1 \mathrm{~h}$ at $4^{\circ} \mathrm{C}$ and were precipitated by centrifugation. The pellets were washed twice with $0.1 \%$ Triton X-100 in PBS and once in distilled water before being resuspended in reducing sample buffer, boiled for $5 \mathrm{~min}$ and separated by SDS-PAGE.

\section{$2.8 .4-S D S-P A G E$}

Reduced and non-reduced samples were run in $0.75 \mathrm{~mm}$ thick standard $7.5 \% \mathrm{SDS}$ polyacrylamide resolving gels with a 5\% stacking gel. A detailed account of the standard procedures for SDS-PAGE together with the composition of solutions is given in reference 
[227]. In gels where immunoprecipitated samples were analysed ${ }^{14} \mathrm{C}$-labelled molecular weight (MW) standards (Bio-Rad Laboratories) were run on lanes adjacent to the samples. Pre-stained MW standards (Sigma, cat no. SDS-7B) were used for gels to be transferred by Western blotting. Although pre-stained MW standards provide an estimate of the molecular weight of the protein of interest, according to the manufacturer's instructions, they should not be used for MW determinations. Gels to be autoradiographed were fixed for 30 min, impregnated with En ${ }^{3}$ Hance (New England Nuclear, Boston, MA, U.S.A.) and dried. Autoradiography was carried out at $-70^{\circ} \mathrm{C}$ for $24 \mathrm{~h}$.

\subsection{5-Western blotting}

Unless otherwise indicated lysates for Western blotting were run in SDSpolyacrylamide gels under non-reducing conditions. After SDS-PAGE, proteins were transferred from the gel to nitrocellulose membranes (Sigma or Schleicher and Schuell, Dassel, Germany) by Western transfer using a semi-dry transfer apparatus (BiometraFast-Blot, Biometra, Göttingen, Germany) running at $150 \mathrm{~mA}$ for $1-1.5 \mathrm{~h}$. Transfer buffer consisted of $25 \mathrm{mM}$ Tris base, $92 \mathrm{mM}$ glycine and $20 \%$ methanol. The efficiency of transfer was assessed by migration of the pre-stained molecular weight markers to the membrane and by lack of staining of the gel with Coomassie blue after transfer. The blotted membrane was blocked overnight in Blotto (5\% powdered milk, $0.05 \%$ Tween $20,0.02 \%$ sodium azide in PBS) and was probed with rabbit anti-murine mannose receptor polyclonal (1:500 to $1: 1000)$ or normal rabbit polyclonal as control, followed by alkaline phosphatase-conjugated goat anti-rabbit (Sigma, cat. no. A8025; 1:1000). All washes and antibody dilutions were in Blotto. The bands were developed with 5-bromo-4-chloro-3indolyl-phosphate/nitroblue tetrazolium (BCIP/NBT; 0.05\% BCIP, 0.1\% NBT, 2mM $\mathrm{MgCl}_{2}$ in $50 \mathrm{mM}$ glycine, $\mathrm{pH} 9.5$ ) and the reaction was terminated by washing in distilled $\mathrm{H}_{2} \mathrm{O}$. 


\subsection{6 - Preparation of a $F\left(a b^{\prime}\right)_{2}$ fragment from anti-mouse mannose receptor antiserum}

Rabbit antiserum against murine mannose receptor proved to be a poor reagent for immunofluorescence because of high background. In an attempt to decrease background due to $\mathrm{Fc}$ receptor binding, a $\mathrm{F}\left(\mathrm{ab}{ }^{\prime}\right)_{2}$ fragment of the polyclonal was made. The procedure for isolating IgG and making the $F\left(a b{ }^{\prime}\right)_{2}$ fragment is described in detail in reference [227]. Briefly, whole serum IgG was purified from the anti-mannose receptor antiserum or from control rabbit serum by affinity chromatography on protein A-agarose columns. After checking purity by SDS-PAGE, the antibody-containing fractions were pooled and were digested with pepsin (Sigma) for $10 \mathrm{~h}$ at $37^{\circ} \mathrm{C}$. $\mathrm{F}\left(\mathrm{ab} \mathrm{b}_{2}\right)_{2}$ fragments were separated from undigested IgG and from $\mathrm{Fc}$ fragments by passing through the protein A-agarose column once more. The resulting preparations were checked by SDS-PAGE under both reducing and non-reducing conditions and found to have the molecular weight characteristics of $\mathrm{F}(\mathrm{ab})_{2}$ fragments. Anti-mannose receptor activity of both purified IgG and $\mathrm{F}\left(\mathrm{ab} \mathrm{b}^{\prime}\right)_{2}$ fragments was preserved, as assessed by Western blotting of J774E protein. 


\section{Chapter 3}

\section{Freshly isolated murine Langerhans cells are phagocytic}

in vitro

\section{1 - Introduction}

3.2 - Freshly isolated Langerhans cells are phagocytic in vitro

3.3 - Flow cytometric analysis of association between fluorescent particles and LC

3.4 - Strain differences in the extent of zymosan uptake

3.5 - Comparison of Langerhans cells and macrophages

3.6 - Summary 


\section{1 - Introduction}

It is clear that members of the DC family can, at some stage, acquire soluble antigens delivered in vivo, such as contact sensitising agents applied to the skin, and antigens delivered in aerosolised form into the lung and airways, or injected directly into the gut, and these cells can induce immunity after adoptive transfer to naive recipients; similar observations have been made for splenic DC. In addition, soluble tracers have been visualised within LC in situ after intradermal or intravaginal administration, or after incubation with LC in vitro (reviewed in chapter 1). However, the phagocytic capability of these cells has been considerably less clear. There is evidence that VC, for example, transport antigens delivered intradermally or from the gut and may contain erythrocytes and/or other cell debris, but the general consensus is that these cells, and isolated lymphoid DC are non-phagocytic in vitro (chapter 1). For adaptive immune responses to be mounted against pathogenic organisms, such as bacteria and yeasts, it would seem necessary that members of the DC family should be phagocytic at some stage in their life cycle. Since non-lymphoid DC in situ are thought to be primarily involved in antigen acquisition it might be expected that they, rather than mature lymphoid DC, would have the greatest potential to ingest particles.

These considerations led us to undertake a detailed study of the phagocytic capacities of freshly-isolated LC as prototypes for immature DC. To investigate the phagocytic capacity of fresh LC we developed an in vitro model where unfractionated, freshly isolated bulk epidermal cell suspensions were incubated with a variety of target particles. Langerhans cells in these cell suspensions were subsequently identified by immunofluorescent staining for MHC class II antigens and were examined for evidence of phagocytosis. The advantage of this approach is that no purification of LC prior to the assay is necessary. Freshly-isolated LC make up $1-4 \%$ of whole EC suspensions and their purification is time-consuming and laborious [140] and could lead to inadvertent selection 
of particular sub-populations. Purification of LC becomes considerably easier after about $12 \mathrm{~h}$ in culture when the density of cells decreases and they can be enriched by centrifugation on dense BSA columns [120]. Thus, for microscopical observations, nonadherent EC were often fractionated over dense BSA after incubating with particles for $\geq 12 \mathrm{~h}$, resulting in an enrichment of $10-40 \% \mathrm{LC}$ in the low density fraction and $<1 \%$ in the high-density pellet. Besides allowing effective enrichment of LC, long incubations also maximised the chances of detecting phagocytic activity by LC; in pilot experiments, it was apparent that these cells were not traditional phagocytes and required considerably longer than $M \varnothing$ to ingest particles. Finally, a further advantage of using unpurified LC was that they needed not be cultured in the presence of exogenous cytokines since these were naturally produced by the contaminating keratinocytes [139, 140]. Purified LC, on the other hand, require GM-CSF and/or TNF- $\alpha$ to remain viable in culture [139].

The panel of phagocytic markers tested was chosen from a long-established list of particles that have been used in the study of phagocytosis by mononuclear and polymorphonuclear phagocytes. This panel included two types of gram-positive bacteria (S. aureus and C. parvum) and yeast-derived particles (zymosan) as examples of microorganisms typically encountered by the immune system, and latex beads $(0.5,1$ and $3 \mu \mathrm{m})$, often thought to act as indicators of "non-specific" phagocytosis. In contrast to these particles, which are internalised via poorly defined phagocytic receptors [191], IgGcoated and $\mathrm{C} 3 \mathrm{bi}$-coated sheep erythrocytes were used as markers for specific phagocytosis via Fc $\gamma R$ and $C R 3$ receptors, respectively, which are known to be present on fresh LC. Many of these phagocytic markers were made fluorescent to ease visualisation of uptake, either by conjugating to FITC (bacteria, zymosan) or, in the case of latex beads, by purchasing commercially available fluorescent particles. Since both the dye in the fluorescent latex beads and FITC emit in the 520-550 nm (yellow/green) region of the spectrum upon excitation by UV light, labelling of the particles did not interfere with labelling of MHC class II antigens on LC using dyes fluorescing in the $580-630 \mathrm{~nm}$ (orange/red) region (Phycoerythrin or Texas Red) 
A combination of methods was used to assess uptake of different particles by fresh LC. Fluorescence microscopy, coupled with phase contrast or Nomarski differential interference contrast light microscopy, was the primary tool in establishing the phagocytic potential of fresh LC. Electron microscopy confirmed the light microscopy observations and flow cytometry was used for many of the quantitation experiments. Together, the observations reported in this chapter provide evidence that freshly-isolated LC are phagocytic in vitro. 


\section{2 - Freshly isolated Langerhans cells are phagocytic in vitro}

Two-colour UV microscopical examination of freshly-isolated EC incubated for $\geq 12 \mathrm{~h}$ with fluorescent particles revealed that the majority of MHC class II-positive cells (LC) within these cultures were associated with particles (fig. 3.1). LC appeared to have phagocytosed C. parvum (fig. 3.1a,b) and S. aureus (fig. 3.1e,f,i) as indicated by the presence of discrete fluorescent spots associated with the cells (fig. 3.1b,f). In contrast to the dim fluorescence of bacterial particles, the bright fluorescence of latex particles associated with LC was unmistakable (fig. 3.1c,d). LC also phagocytosed zymosan (fig. $3.1 \mathrm{~g}, \mathrm{~h})$, and the number of particles internalised (2-8/LC) was easily counted by virtue of its highly fluorescent core (fig. $3.1 \mathrm{~h}$ ), possibly a trypsin-resistant proteinaceous remnant of yeast cytoplasm that binds FITC more avidly than the surrounding carbohydrate coat [228]. To obtain a clearer picture of the localisation of internalised particles within the cell, double red and green exposures of the same cell were made and often showed clustering of the particles in a perinuclear region (fig. $3.1 \mathrm{i}, \mathrm{j}$ ). However, it was not clear whether this represented a single phagosome containing several particles or several phagosomes close together. The latter hypothesis appeared to be true for zymosan (figs. 3.11) and latex beads (see fig. 3.2 b-d). It was noted that not all LC had particles associated with them (fig. $3.1 \mathrm{~g}, \mathrm{~h}$ ) even though the great majority did (fig. 3.1c,d). In addition to LC, other class IInegative cells were also found associated with particles (fig. 3.1a-d) and these were tentatively identified as keratinocytes on the basis of their appearance under phase contrast. These observations are representative of over 10 experiments. 

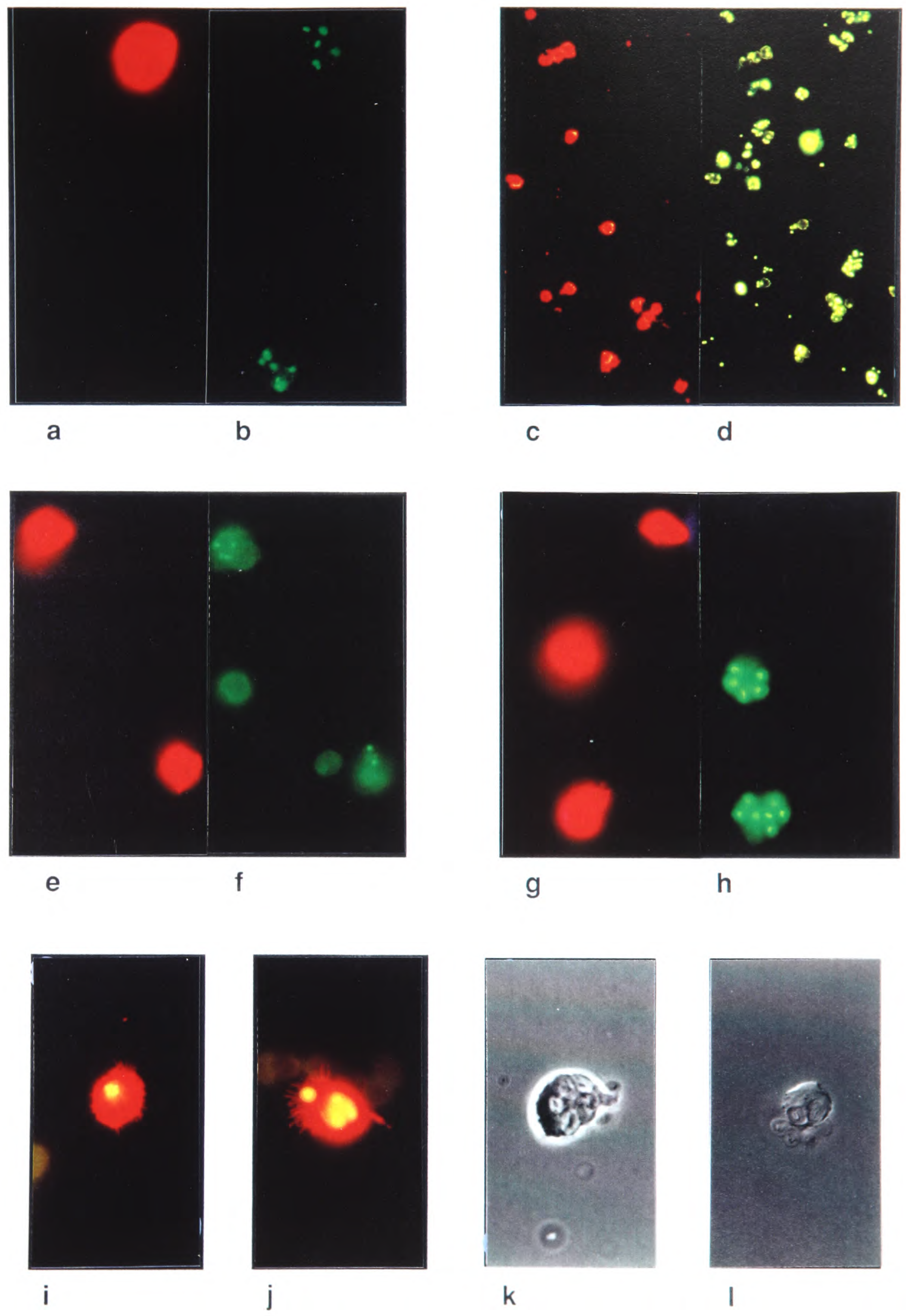
Fig. 3.1: UV light microscopy analysis of particles associated with LC. Bulk EC from C57Bl/6 mice were pulsed with various fluorescent particles for $18 \mathrm{~h}(\mathrm{a}, \mathrm{b}, \mathrm{e}-\mathrm{j})$ or $12 \mathrm{~h}$ $(\mathrm{c}, \mathrm{d}, \mathrm{k}, \mathrm{l})$ immediately after isolation. Low density cells were stained for Ia with Texas Red $(\mathrm{a}, \mathrm{b}, \mathrm{e}-\mathrm{j})$ or PE (c,d,k,l). Particles: a) and b) FITC-C. parvum, c) and d) $1 \mu \mathrm{m}$ fluorescent latex beads, e), f) and i) FITC-S. aureus, g), h ), j-l) FITC-zymosan. Cells were photographed at an initial magnification of $x 1000(a, b, e-1)$ or $x 400(c, d)$. l) and $k$ ) are phase and differential interference contrast micrographs, respectively, of zymosan associated with LC. All other pairs of micrographs (a-h) are red (left) and green (right) fluorescence images of the same field. $\mathbf{i}$ ) and $\mathbf{j}$ ) are double exposures (red and green) of a single cell containing $S$. aureus and zymosan, respectively, and appear yellow where the two colours are superimposed.

Internalisation of zymosan was clearly differentiated from binding to the cell surface by phase contrast (fig. $3.1 \mathrm{k}$ ) and Nomarski differential interference contrast (fig. 3.11) microscopy and revealed that all LC that were associated with particles had internalised at least some, if not most, of them. Similar distinctions between bound and internalised particles were possible for all particles tested except in the case of $3 \mu \mathrm{m}$ latex beads where true internalisation was difficult to distinguish from binding. To confirm that internalisation had occurred, fresh epidermal cell suspensions were incubated for $12-18 \mathrm{~h}$ with latex beads of various sizes $(0.5-3.5 \mu \mathrm{m})$, or with zymosan, fractionated over dense BSA to enrich for LC and processed for transmission electron microscopy (fig. 3.2). LC were readily identified by morphology and distinguished from keratinocytes which, for example, possess a distinct rim of peripheral intermediate filaments (not shown). Birbeck granules (BG), a marker for LC in many species, cannot be used as a criterion because they are not readily detectable in the mouse [185]. ${ }^{1}$ From this analysis it was clear that fresh LC can indeed phagocytose latex microspheres ranging in size from $0.5 \mu \mathrm{m}-3.5 \mu \mathrm{m}$ (fig. $3.2 \mathrm{a}-$ c), apparently within membrane-bounded phagosomes (fig. 3.2d). Zymosan particles phagocytosed by LC assume an irregular shape with a distorted electron-dense core (fig.

\footnotetext{
${ }^{1}$ Birbeck granules are also lost during LC maturation $[120,133]$ and, for murine LC, very few remain after $12 \mathrm{~h}$ of culture (personal observations and $\mathrm{N}$. Romani, personal communication).
} 
3.2e) in marked contrast to the more oval and regular appearance of extracellular particles (not shown). This is, presumably, a result of intracellular degradation and was observed in four independent electron microscopy preparations. Discoloured areas within the latex beads (fig. 3.2a-d) do not represent degradation but, instead, artefactual shrinkage and extraction of the latex during processing for electron microscopy as noted by others [204, 229]. Latex and zymosan particles were also visualised within some keratinocytes (not shown) in agreement with UV microscopy experiments (see above) and published observations on uptake of latex beads by keratinocytes [204].

Fig. 3.2: Confirmation of phagocytosis by LC using electron microscopy. Transmissior electron micrographs through LC containing a) $3.41 \mu \mathrm{m}$, b) $0.92 \mu \mathrm{m}$, c) and d) $0.49 \mu \mathrm{m}$ latex particles (LP) or e) zymosan (Z). Magnification: a) x16000, b) x13000, c) x13000, d) $x 24000$, e) $x 12000$. Although BG were not seen in any of the cells shown here, they were occasionally noted in cells of identical morphology. 


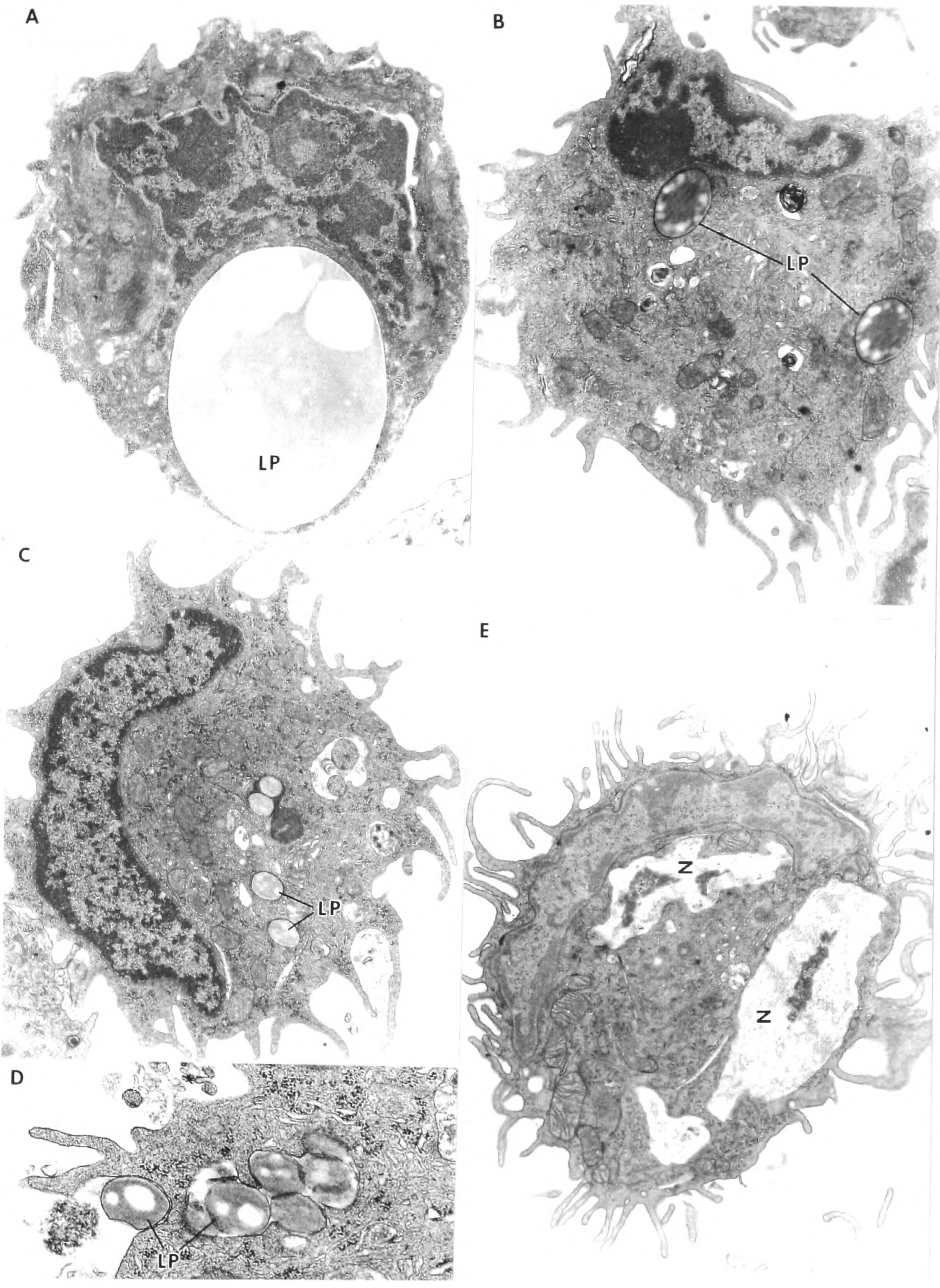


It was of interest to determine whether LC can phagocytose particles via FC receptors for IgG (FcyR) or via complement receptors (type 3, CD11b/CD18) which are expressed by fresh LC (see chapter 1). Therefore, sheep erythrocytes (SRBC) were opsonised with IgG anti-SRBC antibodies (IgG-SRBC), or with IgM anti-SRBC and human complement $\left(\mathrm{C}^{\prime}-\mathrm{SRBC}\right)$ as described in the methods and tested in a standard long incubation assay. However, in at least six consecutive experiments, no binding or uptake of these opsonised erythrocytes by fresh LC was observed as shown on fig. 3.3a for IgGSRBC. In contrast, thioglycollate-elicited peritoneal macrophages (thio-M $\varnothing$ ) which were used as a positive control in four of these experiments, phagocytosed IgG-SRBC (fig. 3.3c) and bound C'-SRBC very tightly (not shown). Because we used cell fractionation over BSA to enrich for LC at the end of each assay, it remained possible that any LC containing SRBC were in the high density fraction. However, careful examination revealed that in all experiments $>95 \%$ LC were recovered in the low density fraction as usual, and none of the LC in the high density fraction contained erythrocytes. The inability of LC to phagocytose erythrocytes does not appear to be due simply to the relatively large size of these particles $(4-6 \mu \mathrm{m})$ because LC phagocytose heat-killed S. cerevisiae cells of a similar size (personal observations; not shown). Furthermore, erythrocytes are often present within Langerhans cell migrating from human skin explants (C.P. Larsen, personal communication) and, occasionally, in those from mouse (personal observations), and have been reported in veiled cells from the lymph of sheep [93]. These observations suggested that LC might phagocytose autologous (effete?) erythrocytes. To examine whether this occurred in vitro, mouse splenic RBC were incubated in autologous serum and tested on EC or on M $\varnothing$ cultures. No RBC were taken up by LC although they were bound to some extent by $M \varnothing$, either because of opsonisation with complement or with natural antibodies (not shown).

To test the hypothesis that FITC conjugation of itself may be responsible for the observed uptake of fluorescent particles, we made use of the observation that LC do not bind or phagocytose sheep erythrocytes. Formalin-fixed sheep erythrocytes were 
conjugated to FITC overnight in carbonate buffer, $\mathrm{pH} 9.5$, or incubated in buffer alone as a control and tested on $\mathrm{M} \varnothing$ and EC cultures. The results in fig. 3.4 clearly demonstrate that, although M $\varnothing$ bind and phagocytose aldehyde-fixed SRBC (fig. 3.4a,b), conjugation to FITC does not affect the extent of uptake (compare figs. $3.4 \mathrm{a}, \mathrm{b}$ with $3.4 \mathrm{c}, \mathrm{d}$ ). More importantly, LC did not bind or phagocytose either the control (fig. 3.4e,f) or the FITCconjugated (fig. $3.4 \mathrm{~g}, \mathrm{~h}$ ) erythrocytes, suggesting that FITC conjugation of itself it is not sufficient to promote particle phagocytosis. Phagocytosis of aldehyde-fixed SRBC by M $\varnothing$ has been reported $[196,201]$ although the receptors involved have not been identified [191]. 


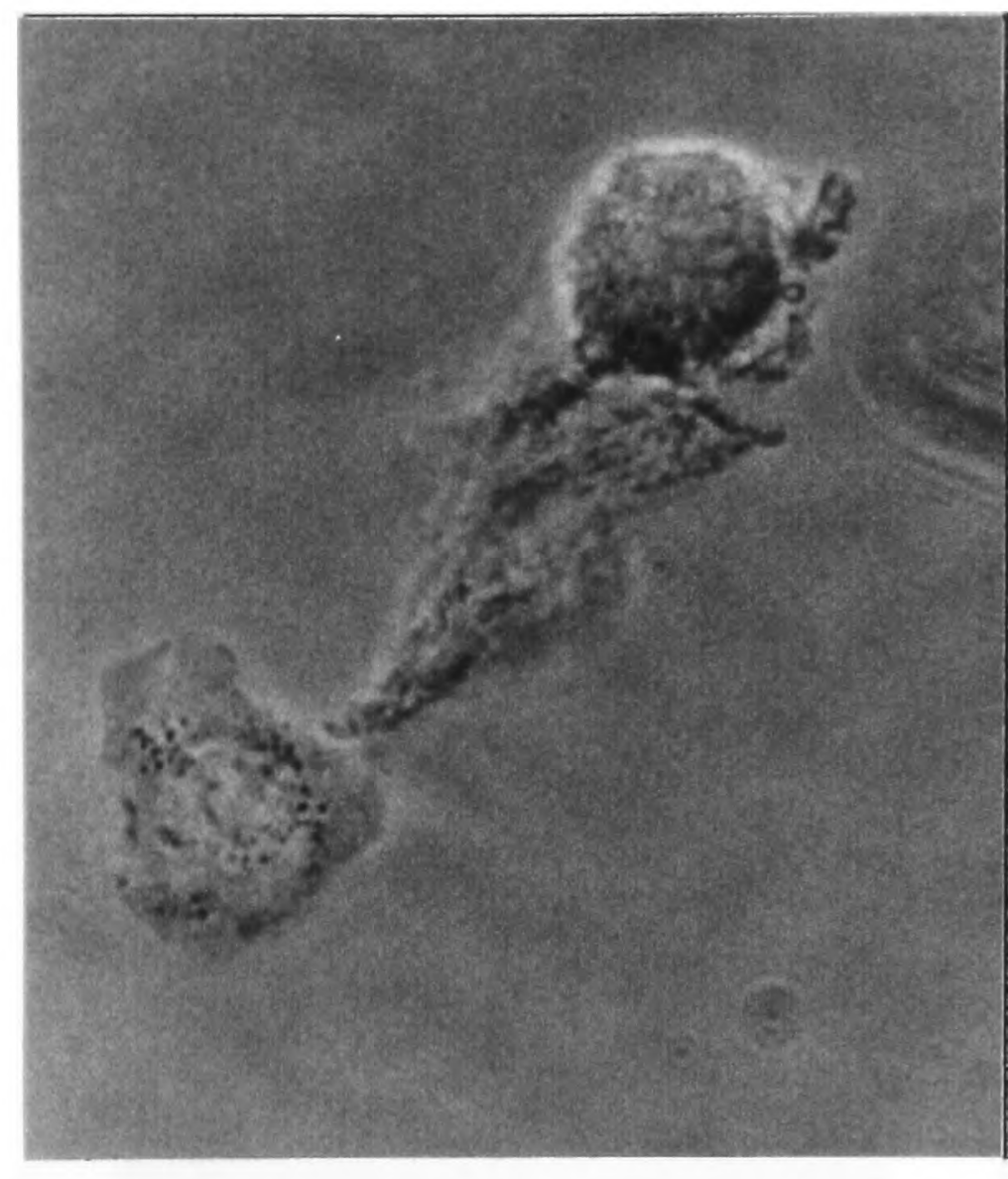

a

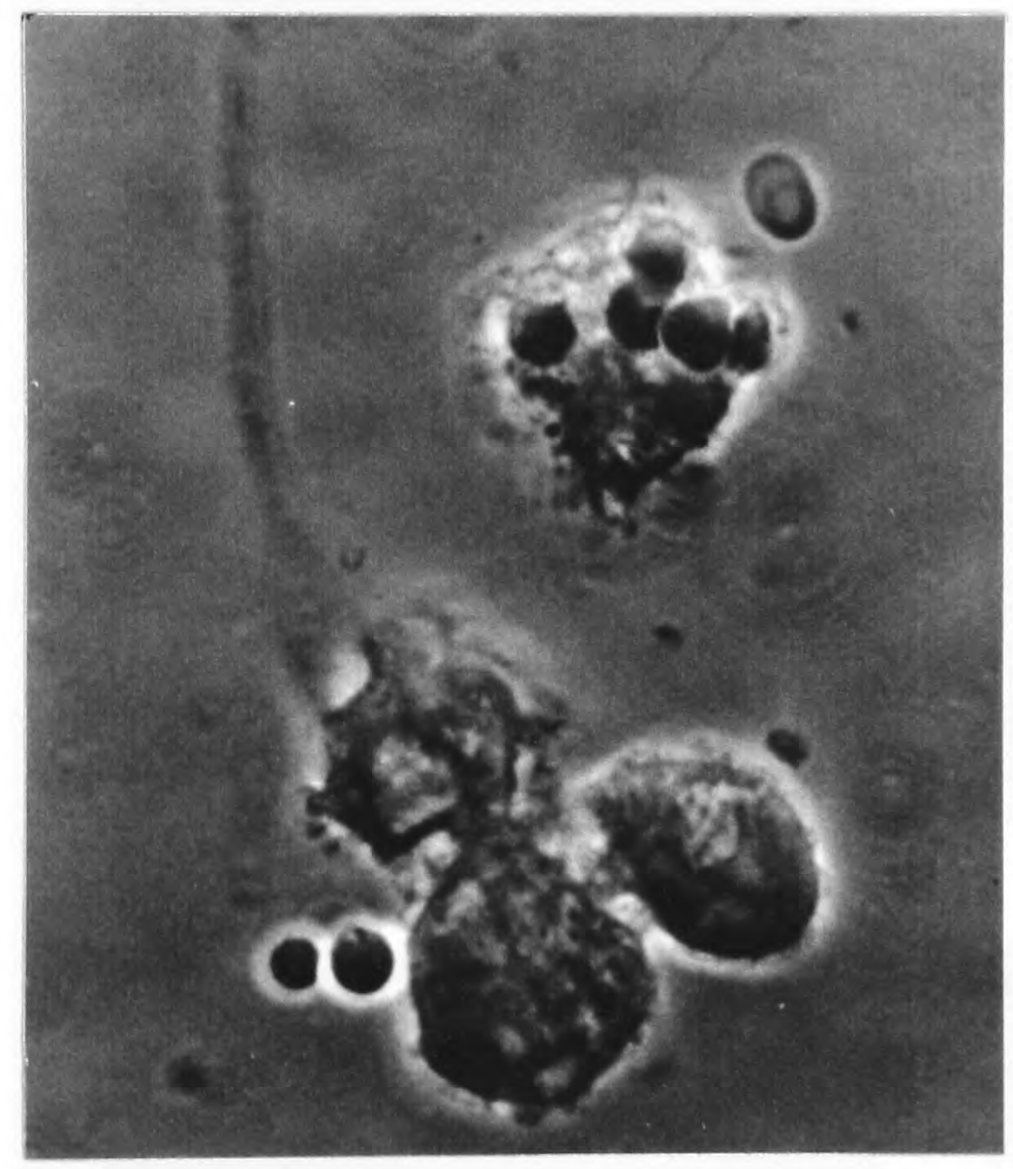

C

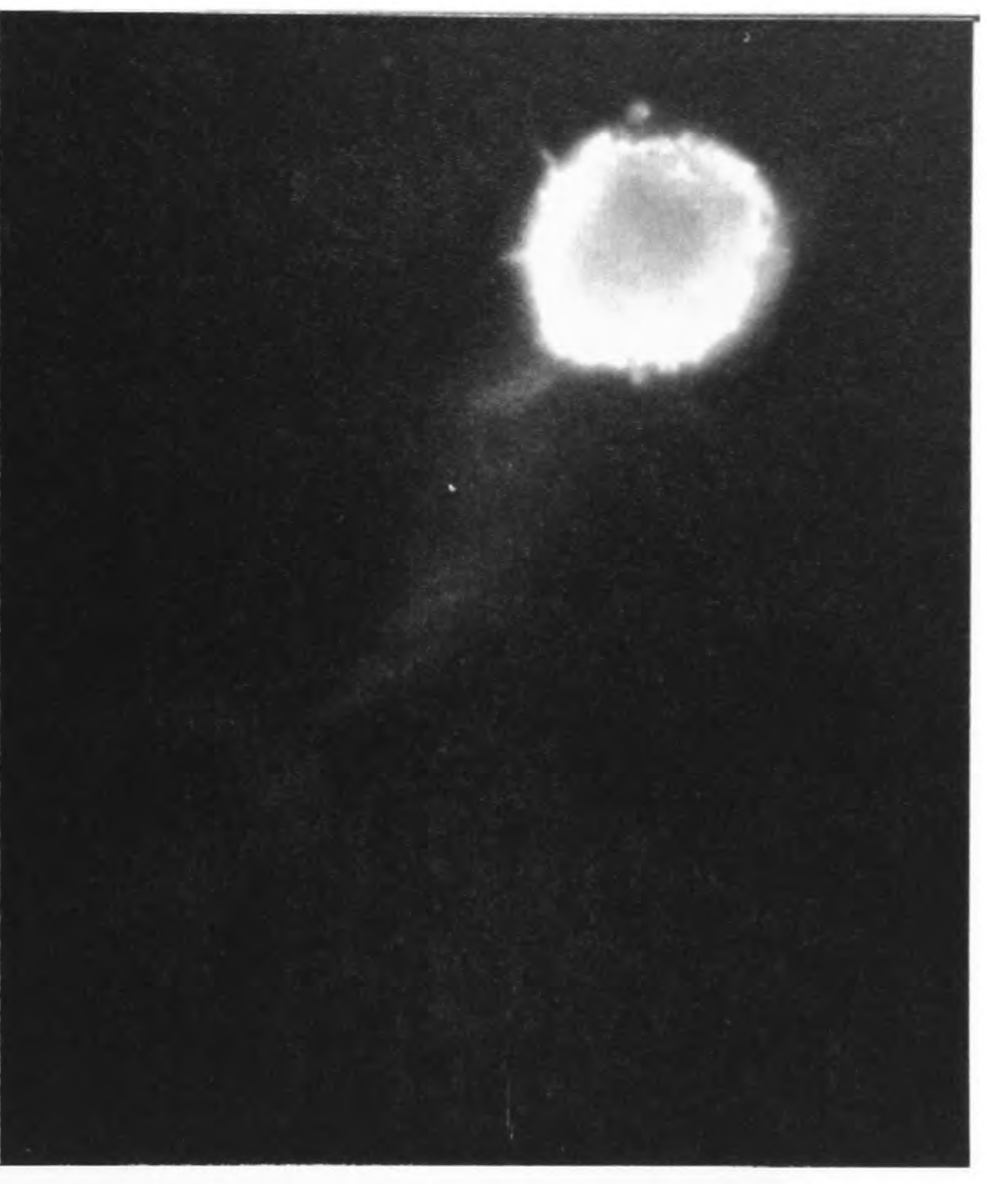

b

Fig. 3.3: Freshly isolated LC do not phagocytose IgG-coated sheep erythrocytes. Fresh EC $(a, b)$ or resident peritoneal $\mathrm{M} \varnothing(\mathrm{c})$ were incubated for $16 \mathrm{~h}$ with IgG-opsonised SRBC. LC in the low density fraction of EC, identified by staining for MHC class II antigens (b), never showed signs of having ingested IgG-SRBC (a). About $30 \%$ of resident $\mathrm{M} \varnothing$ phagocytosed IgG-SRBC in this experiment, acting as a positive control (not shown). The M $\varnothing$ in c) are from a separate experiment but are representative of the results obtained in this and 3 other experiments. No LC $(<1 \%)$ were found in the high-density EC fraction. 
Phase contrast

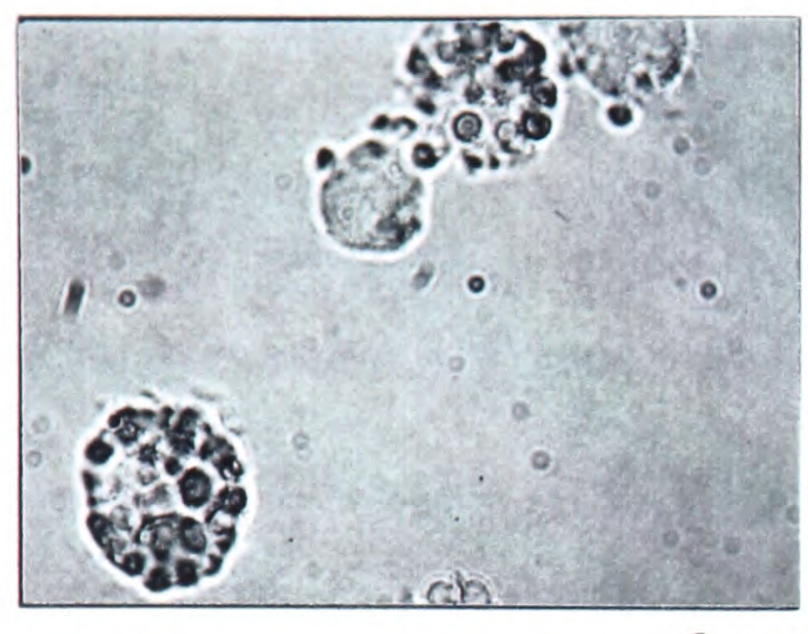

a

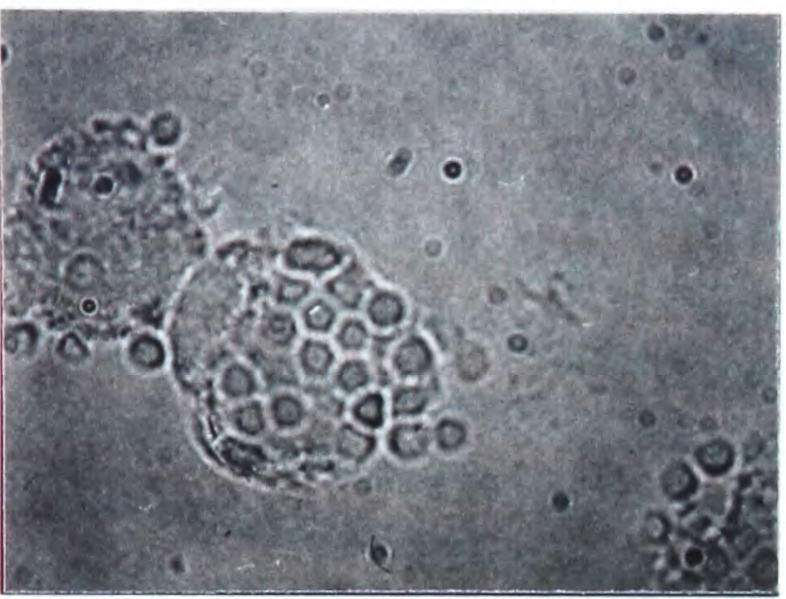

C

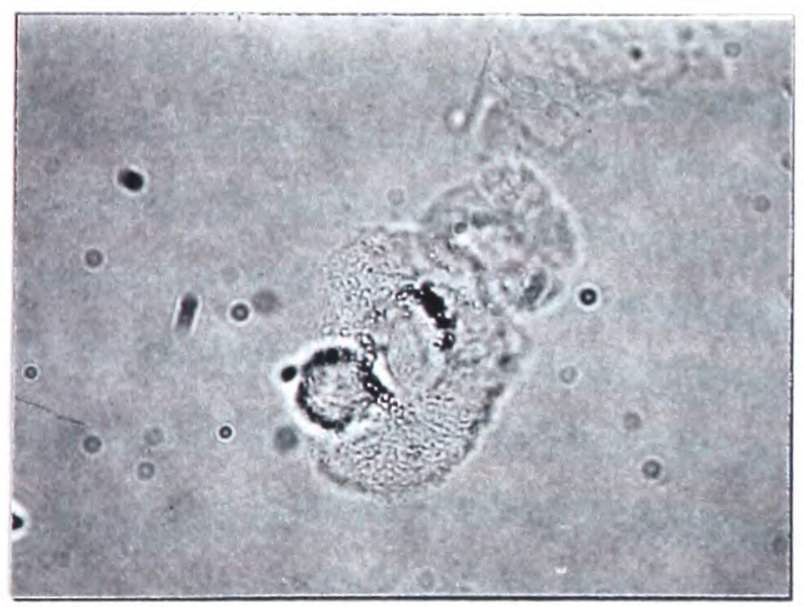

e

Control SRBC

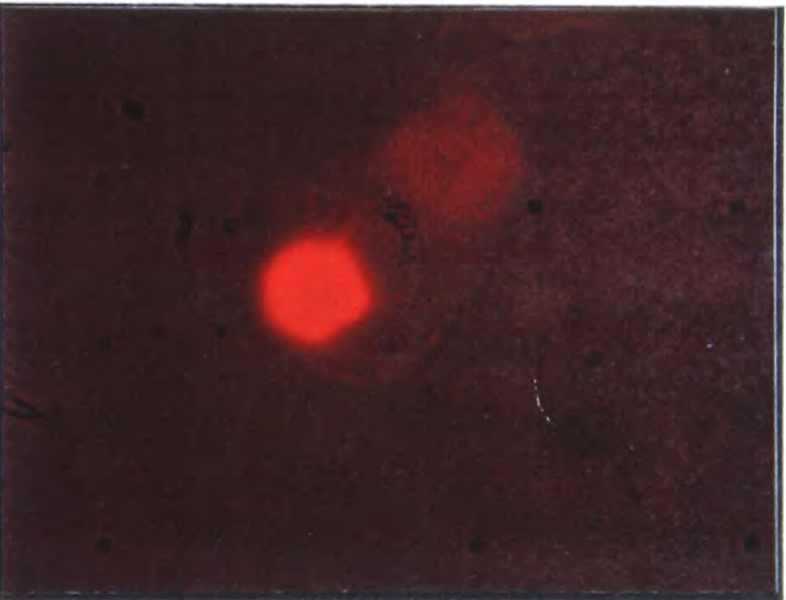

$f$

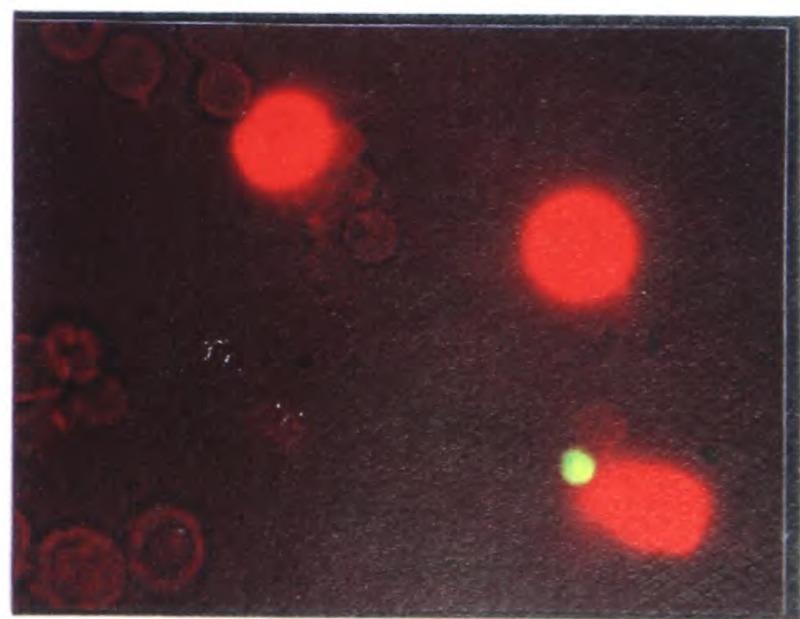

g
Fluorescence

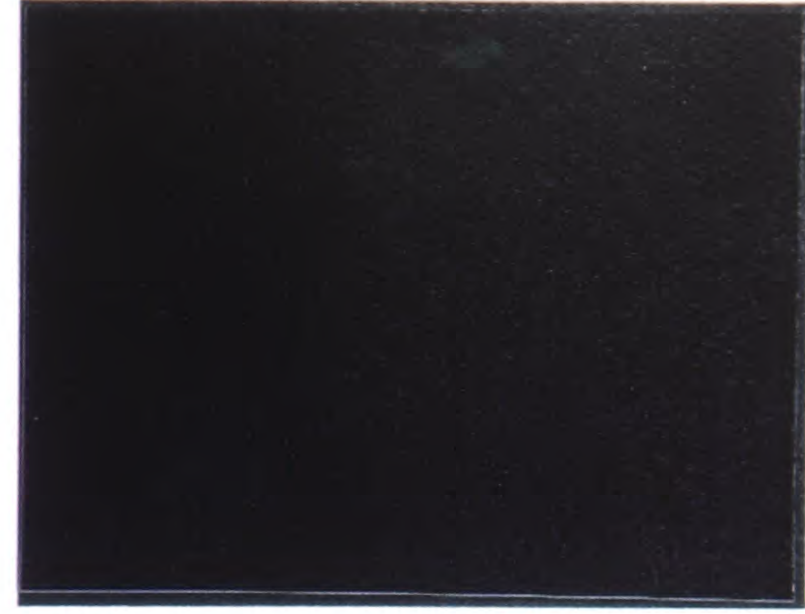

b

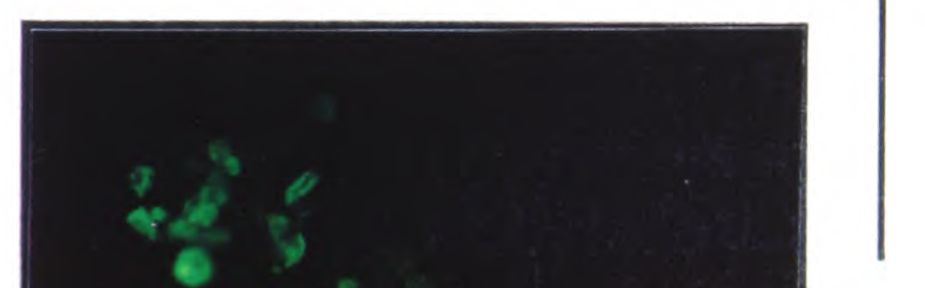

8

d

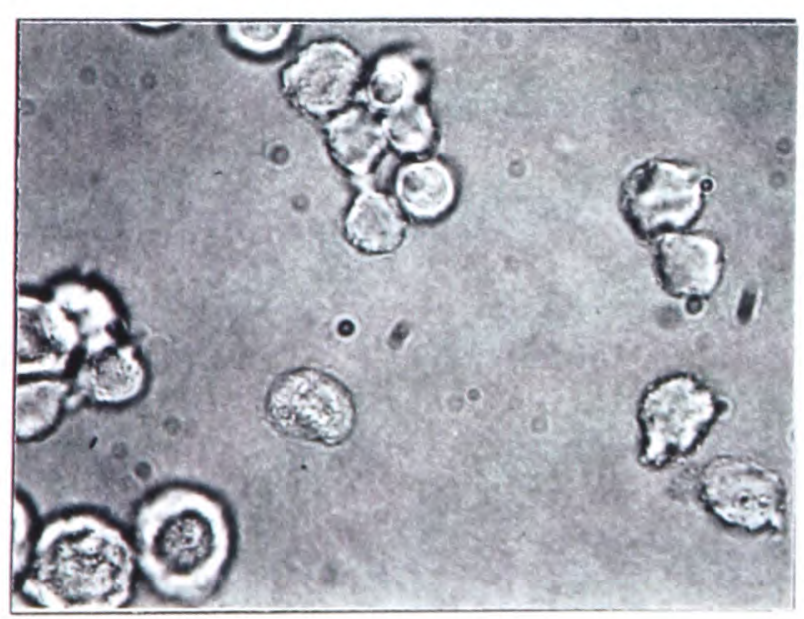


Fig. 3.4: Conjugation to FITC does not promote the uptake of SRBC. Resident peritoneal M $\varnothing(\mathbf{a}-\mathbf{d})$ or fresh EC suspensions (e-h) were incubated with control aldehydefixed SRBC $(a, b, e, f)$ or FITC-SRBC $(c, d, g, h)$ for $16 \mathrm{~h}$. The low-density fraction of EC was stained for MHC class II antigens with Texas Red to identify LC (f,h). All photographs were taken at $\times 1000$. The right-hand photograph in each pair shows the fluorescence image for the phase contrast field shown on the left. Filters: b,d: green; f: red; h: both (double exposure).

\section{3 - Flow cytometric analysis of association between fluorescent particles and LC}

Quantifying phagocytosis by LC using microscopic techniques proved to be very difficult since the cells were present in low numbers and had to be identified by fluorescence prior to determining the number of particles present. However, the experimental set-up proved to be ideal for dual-fluorescence flow cytometry, enabling faster and more reliable data collection (see chapter 2, section 2.5.1). An example of this analysis is given in fig. 3.5 for FITC-conjugated $S$. aureus. Whole epidermal cells stained for MHC class II antigens (Ia) contained an $\mathrm{Ia}^{+}$population of LC (fig. 3.5a). When the cells were incubated with FITC-S. aureus both $\mathrm{Ia}^{+}$and $\mathrm{Ia}^{-}$cells were associated with the bacteria (fig. $3.5 \mathrm{~b}$ ). To analyse the LC population in more detail, the $\mathrm{Ia}^{-}$population was gated out and a uniformly $\mathrm{Ia}^{+}$population was acquired from the control EC without particles (fig. 3.5c, dotted line) and from the test sample (solid line). The profile of the test population on the FITC channel (fig. 3.5d, solid line) shows that $S$. aureus is associated with $95.5 \%$ of LC as compared to the control LC (dotted line) incubated without particles. Whilst, in principle, this assay cannot differentiate between true internalisation of particles and binding to the cell surface, in practice, complementary microscopic observations confirmed that cells prepared for flow cytometry experiments had few attached particles, possibly due to vigorous vortexing during staining. For all phagocytic markers tested, LC that were associated with particles had phagocytosed at least some of them and, therefore, the percentage of LC associated with particles as measured by FACS is, in practice, 
equivalent to the percentage of LC that are phagocytic.
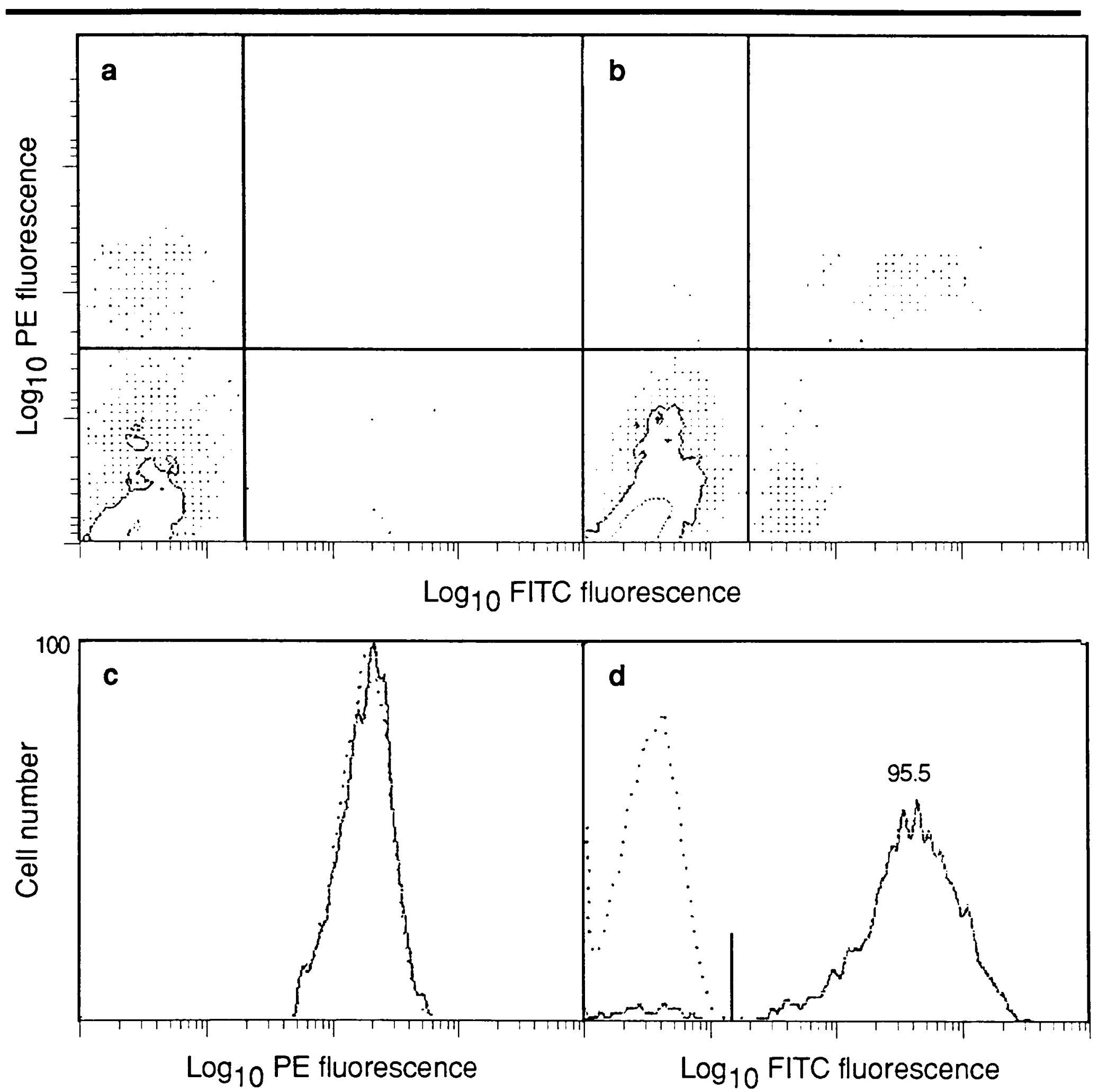

Fig. 3.5: Flow cytometry assay for phagocytosis by LC. C57Bl/6 EC were cultured from $0-12 \mathrm{~h}$ with or without a saturating concentration of FITC-S. aureus. The cells were fixed and prepared for flow cytometry as described in chapter 2. a) control EC stained for Ia with PE, b) S. aureus-pulsed EC stained for Ia with PE, c) PE profile of 3000 gated LC from control (dotted line) and $S$. aureus-pulsed samples (solid line), d) FITC profile of the populations in c). See text and section 2.5.1 for details. 
A series of kinetic studies was performed looking at dose- and time-dependence of uptake by LC plated on tissue culture dishes. Uptake of zymosan by C57Bl/6 LC, as determined by flow cytometry, was saturable at doses of $5 \mu \mathrm{g} / \mathrm{cm}^{2}\left(\approx 10^{6}\right.$ particles $\left./ \mathrm{cm}^{2}\right)$, both in a 90 min assay (fig. 3.6a) and in an overnight incubation (not shown). Since cultures contained around $2 \times 10^{5} \mathrm{EC} / \mathrm{cm}^{2}$, a rough calculation indicates that around five particles of zymosan per LC were required to achieve saturation. This result fits in well with microscopical quantitation of the number of particles internalised per LC (5.2 \pm 3.1 ; see section 3.5) using a 2 -fold saturating dose of $10 \mu \mathrm{g} / \mathrm{cm}^{2}$. FITC did not appear to affect the extent of uptake since, at saturation, about $90 \%$ of the cells were labelled with FITCzymosan (fig. 3.6a), a result which fits in well with results obtained by microscopical scoring of the uptake of unconjugated zymosan (83\% (219 of 264) LC with $\geq 1$ particle of zymosan in one experiment, representative of 6 others). In over 20 experiments using long incubations with a saturating dose of FITC-zymosan, $\geq 85 \%$ of C57Bl/6 LC consistently were associated with particles. Uptake of $1 \mu \mathrm{m}$ latex beads also showed a dose response curve (fig. 3.6b) that appeared to plateau at around 50\% labelled LC but reached 70-100\% labelled LC in many other experiments where long incubations were used (see fig. 3.9, for example). The length of incubation was shown to play a crucial role in the extent of association. Saturation was only observed after about $12 \mathrm{~h}$ continuous incubation with FITC-zymosan (fig. 3.6c) with a slight decrease thereafter, possibly due to degradation (see also sections 4.3 and 4.4 ). 
a
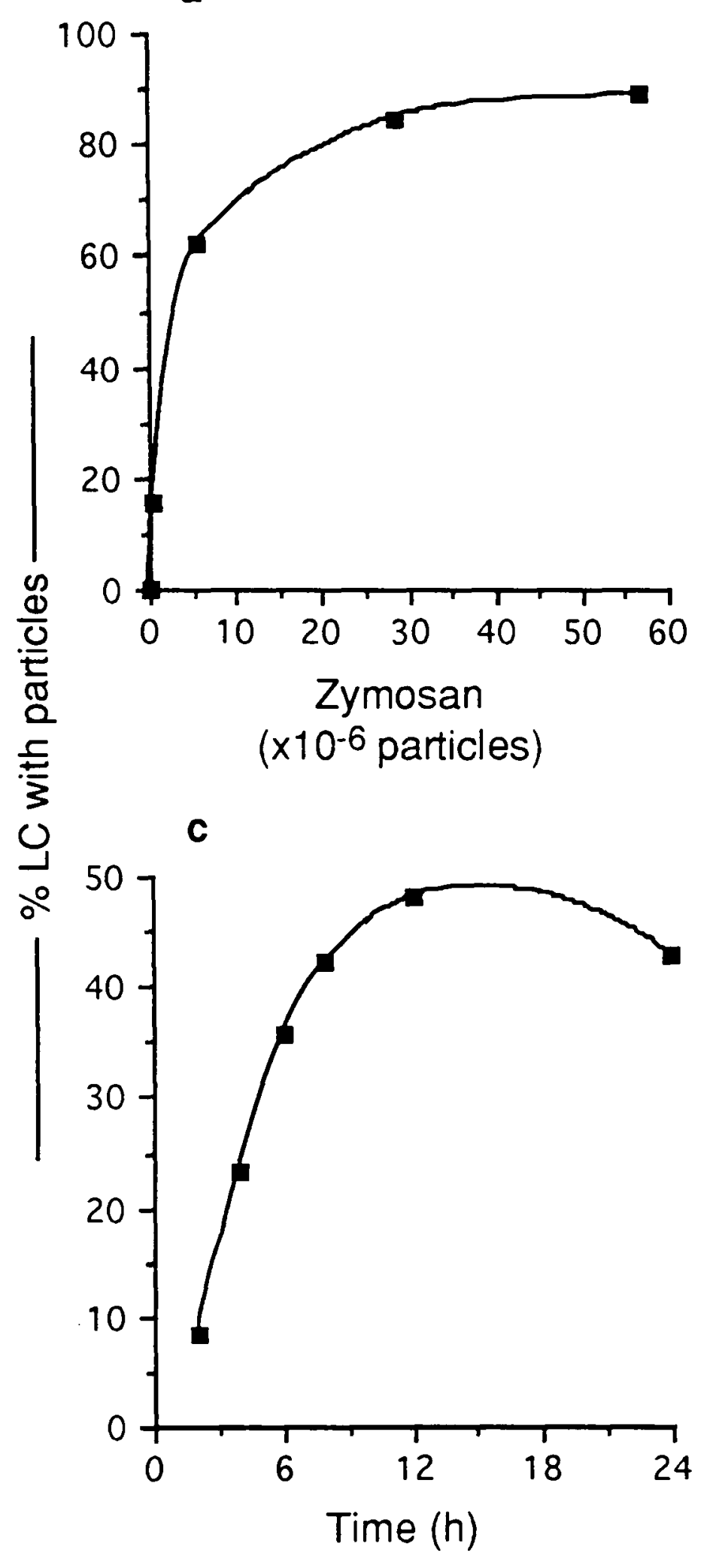

b

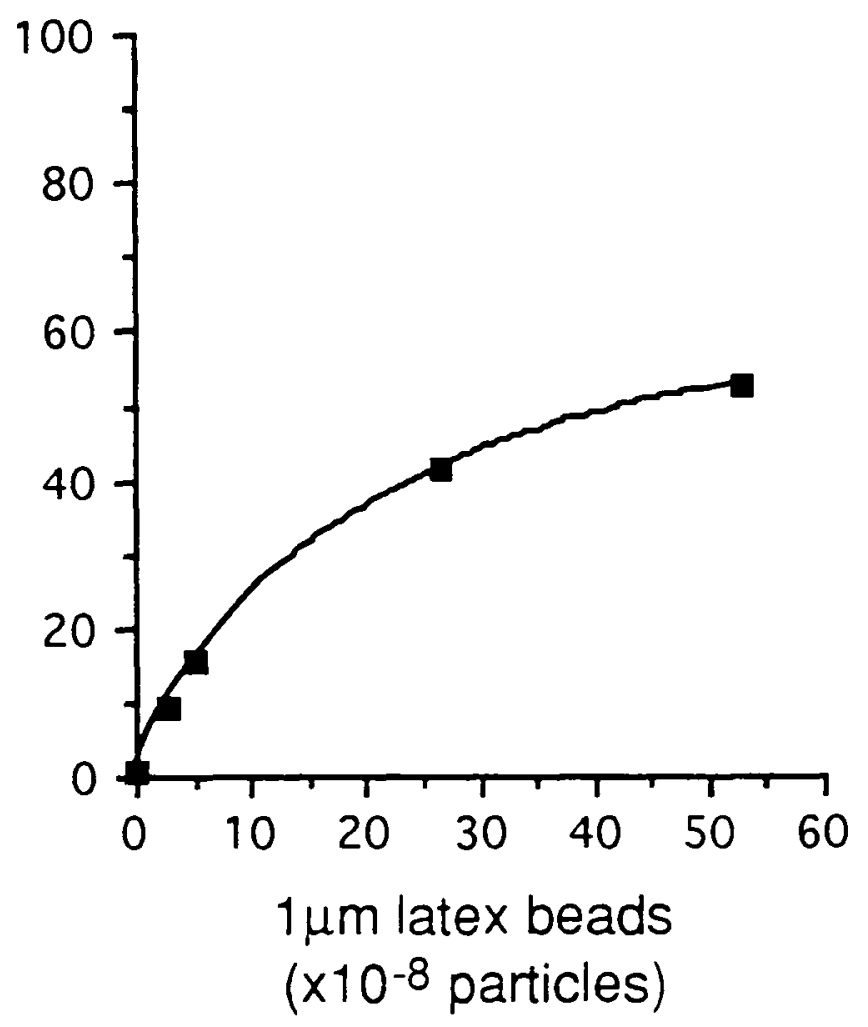

Fig. 3.6: Flow cytometric analysis of particle association with LC. Dose response curves for a) FITC-zymosan and b) $1 \mu \mathrm{m}$ fluorescent latex beads. C57Bl/6 EC were cultured for $6 \mathrm{~h}$ and were pulsed with the indicated number of zymosan or latex particles on $60 \mathrm{~mm}$ tissue culture dishes. Following a 90 min assay the non-adherent cells were harvested, fixed, stained and analysed by FACS as described in the methods. c) time dependence of uptake of zymosan. EC from BALB/c mice were pulsed with $10 \mu \mathrm{g} / \mathrm{cm}^{2}$ FITC-zymosan on tissue culture dishes from the time of plating, harvested at the indicated times and processed and analysed as above.

Next, we used the flow cytometry assay to compare the uptake of different particles by $L C$. Based on the results shown in fig. 3.6 and three other experiments with zymosan 
and/or latex, fresh EC were incubated with bacteria, zymosan or latex beads for $12 \mathrm{~h}$ from the time of plating, and the markers were used at $2 \times 10^{6}$ particles $/ \mathrm{cm}^{2}$, a concentration 2fold greater than that which is saturating for zymosan (fig. 3.6a). The results from two such experiments (fig. 3.7a,b) indicate that the percentage of phagocytic LC varies considerably for different particles, suggesting that the phagocytic ability of LC is heterogeneous, perhaps as a result of heterogeneous expression of particular phagocytic receptors by different cells.

a

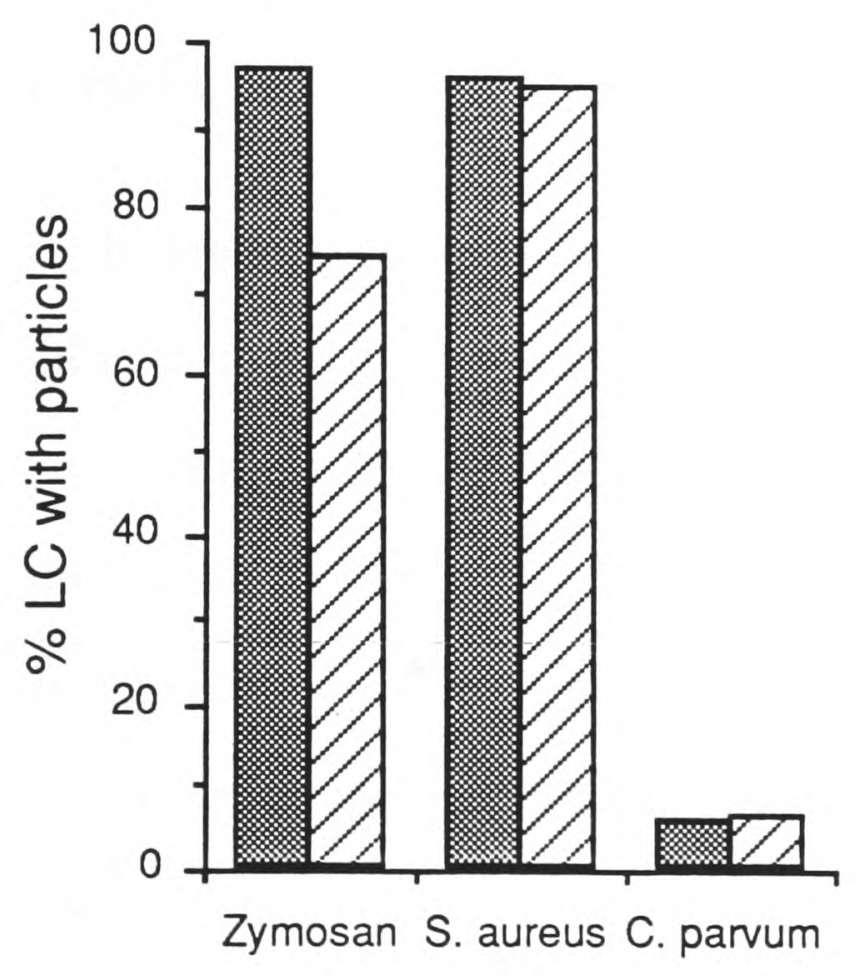

b

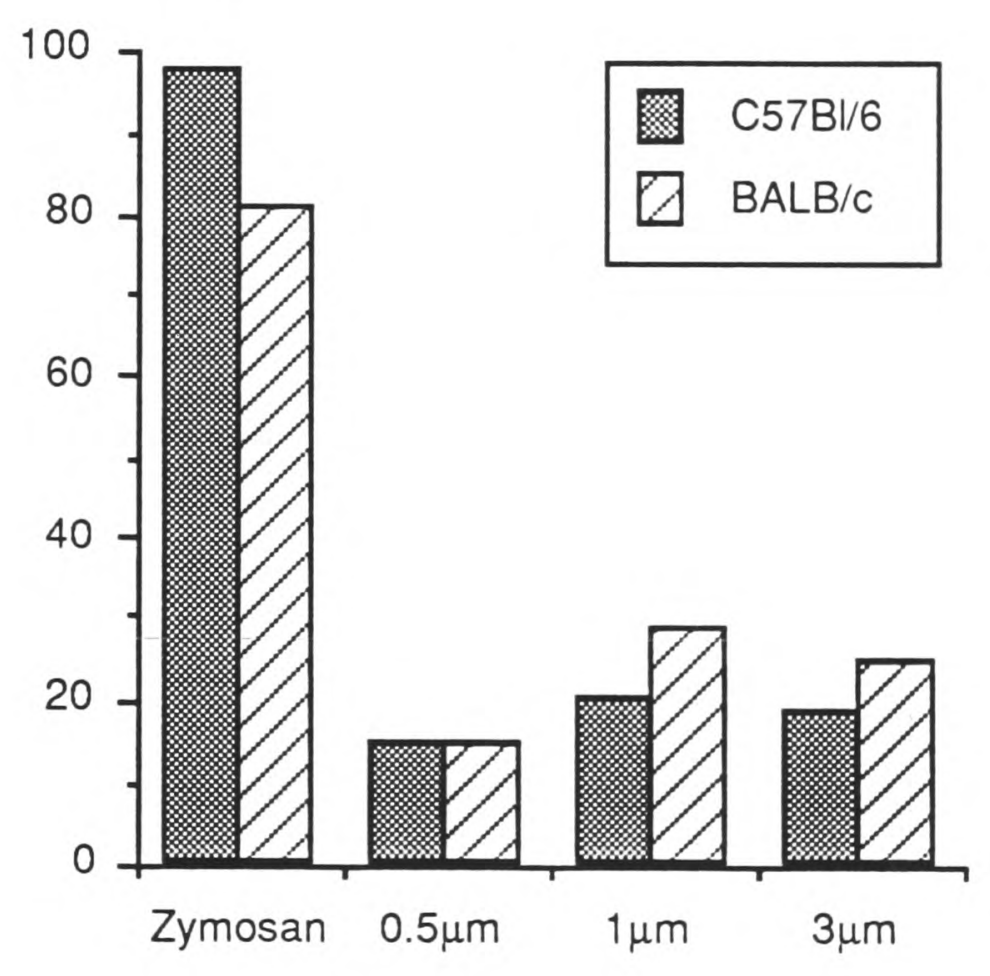

Fig. 3.7: Comparison of the association of different particles with LC. EC prepared from $\mathrm{C} 57 \mathrm{Bl} / 6$ and $\mathrm{BALB} / \mathrm{c}$ mice were incubated from $0-12 \mathrm{~h}$ with $5.7 \times 10^{7}$ of the indicated fluorescent particles on $60 \mathrm{~mm}$ tissue culture dishes $\left(2 \times 10^{6}\right.$ particles $\left./ \mathrm{cm}^{2}\right)$. Non-adherent EC were processed and LC analysed by FACS as described in the methods. a) and b) are from different experiments; zymosan was included in both for comparison.

However, a cautionary note is required here since the use of particles at equal concentrations may be flawed. Differences in mass between different particles determine their distribution within the culture vessel where the assay is performed such that the 
concentration of particles available to the cells is not necessarily a function of their density. For example, because $0.5 \mu \mathrm{m}$ latex beads do not settle to the bottom of the culture vessel to the same extent as the heavier zymosan particles, the actual number of latex particles available to LC is likely to be lower than that of zymosan. This argument can be extended to all particles tested and means that the phagocytic ability for different particles in fig. 3.7 should not be compared directly (it also means that the number of particles necessary to reach saturation cannot be compared between figs. 3.6a and 3.6b). Interestingly, in face of this argument, it would appear that association of LC with S. aureus is extremely avid since similar percentage association was found between those particles and the much larger zymosan particles (fig. 3.7a). However, nothing is known about the relative densities of the two particles and other technical factors could also be responsible.

Instead of using a fixed concentration, the uptake of bacteria and zymosan was compared using the particles at their respective saturating concentrations (fig. 3.8). As in fig. 3.7a, virtually $100 \%$ of LC were associated with zymosan or S. aureus but now, in contrast to the previous experiment, around $50 \%$ of the cells were also associated with $C$. parvum. In addition, the effect of pre-incubating the particles in fresh normal mouse serum (NMS) to induce complement opsonisation via the alternative pathway was also examined. Interestingly, incubation in NMS increased the \% LC associated with opsonised C.parvum to $100 \%$ and an accompanying 5-fold increase in the mean fluorescence of those cells (a measure of the number of particles/cell) was observed. Only a modest difference in mean fluorescence between cells pulsed with opsonised or unopsonised zymosan/S. aureus was seen, possibly reflecting saturation of the uptake process or less efficient activation of the alternative pathway for complement opsonisation by these particles. Alternatively, it is possible that the serum used contained natural and/or specific antibodies against $C$. parvum. These observations were not pursued further. 


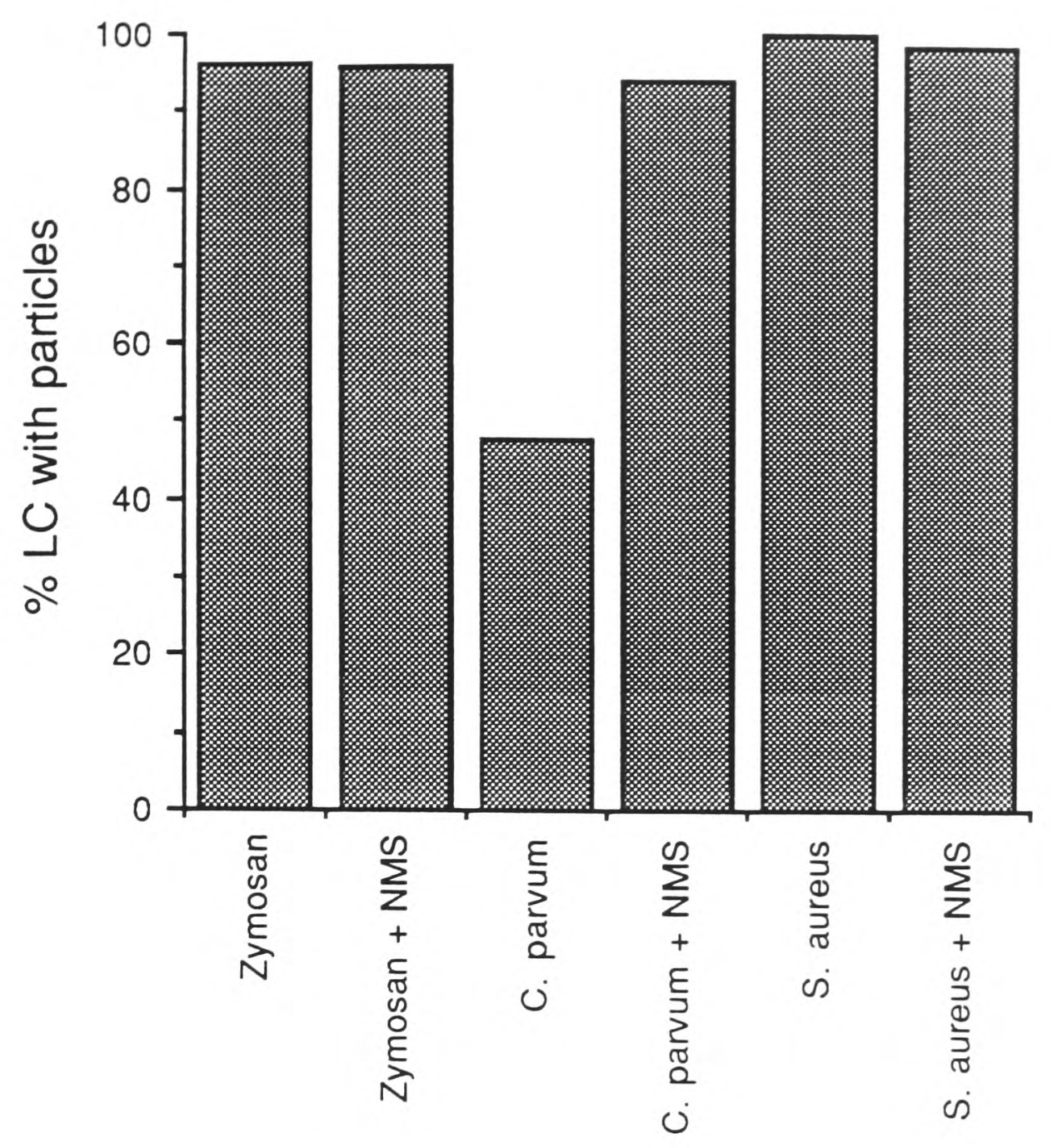

Fig. 3.8: Association of bacteria and zymosan with LC and complement opsonisation. C57B1/6 EC were cultured overnight with saturating concentrations of fluorescent zymosan or bacteria and the extent of particle association with LC was measured by FACS. The saturating concentration for bacteria was taken as that which was saturating for thioglycollate-elicited peritoneal $\mathrm{M} \varnothing$ in a $1 \mathrm{~h}$ assay (not shown). For complement opsonisation, particles were incubated in neat, fresh $\mathrm{C} 57 \mathrm{Bl} / 6$ serum for $30 \mathrm{~min}$ at $37^{\circ} \mathrm{C}$ prior to adding to the cells.

The extent of phagocytosis of latex beads by LC appeared to be inversely related to bead size from microscopic observations but the results presented in fig. $3.7 \mathrm{~b}$ seemed to indicate otherwise. However, because of the problems discussed above, that result may not reflect the true capacity of LC to internalise beads of increasing size. Thus, $3 \mu \mathrm{m}$ beads may artificially be present at a higher local concentration than $0.5 \mu \mathrm{m}$ beads when the same number of beads is added to each culture. A practical demonstration that this may be the case comes from observations made during the experimental procedures: all the $3 \mu \mathrm{m}$ beads pellet with the cells during centrifugation at the end of the assay whilst the majority of 
$0.5 \mu \mathrm{m}$ beads remain in suspension; $1 \mu \mathrm{m}$ beads, as expected, show an intermediate behaviour. Furthermore, observation of the cultures under an inverted microscope clearly showed that, whilst many of the $3 \mu \mathrm{m}$ and $1 \mu \mathrm{m}$ particles were resting on the bottom of the dish, most of the $0.5 \mu \mathrm{m}$ beads remained in suspension. To attempt to compensate for this phenomenon, each culture was pulsed with a fixed concentration of latex instead of a fixed number of beads. The rationale behind this approach was that, for any given concentration of latex, the number of particles is inversely correlated with the size (mass) of the bead; pulsing cells with the same mass/volume of latex should result in equivalent densities of latex sedimenting at the bottom of the culture dish. The results (fig. 3.9) show clear sizedependence of bead association with LC. However, the pulsing rationale is open to debate and these results are included only for comparison with the results in fig. $3.7 \mathrm{~b}$.

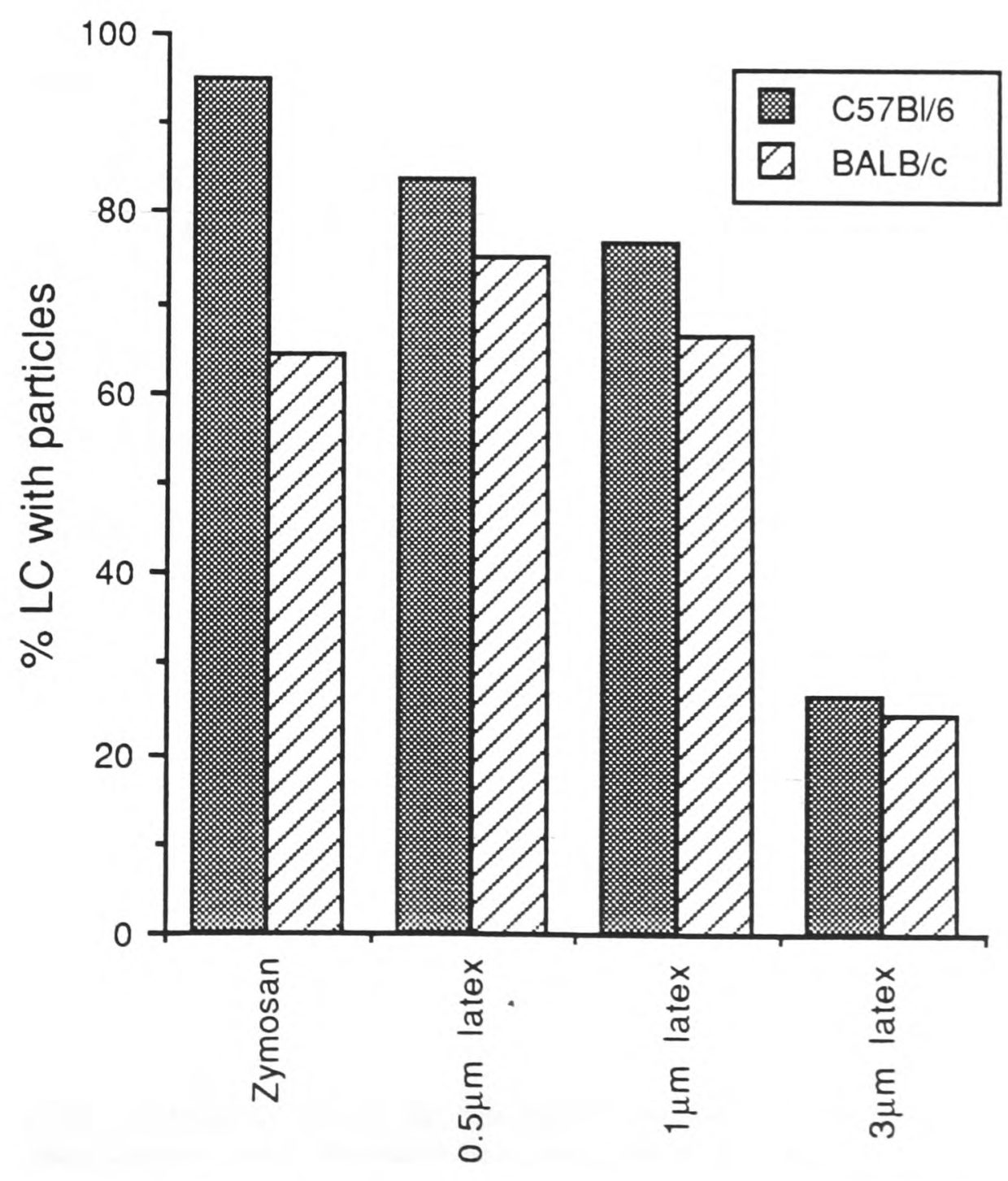

Fig. 3.9: Size dependence of latex bead association with $\mathrm{LC}$ pulsed with a fixed concentration of latex. Fresh $\mathrm{EC}$ prepared from $\mathrm{C} 57 \mathrm{Bl} / 6$ and $\mathrm{BALB} / \mathrm{c}$ mice were 
incubated from $0-12 \mathrm{~h}$ with FITC-zymosan $\left(10 \mu \mathrm{g} / \mathrm{cm}^{2}\right)$ or the indicated sizes of latex beads at $0.005 \%(\mathrm{w} / \mathrm{v})$. Association with LC was measured by FACS.

\section{4 - Strain differences in the extent of zymosan uptake}

When LC from C57B1/6 and BALB/c were compared, an apparent discrepancy in the extent of uptake of zymosan at saturation between the two strains was observed (compare 3.6a and 3.6c, for example). More C57Bl/6 LC were consistently associated with zymosan than those from $B A L B / c$ mice, apparently in a specific manner since other particles did not show such differences (fig. 3.7) or these were more limited in extent (fig. 3.9). In addition to the differences in \% association, phagocytic C57Bl/6 LC always showed a greater mean fluorescence for FITC-zymosan than BALB/C LC, implying that they were associated with a larger number of particles. $\mathrm{C} 3 \mathrm{H} / \mathrm{HeN}$ mice showed an intermediate phenotype as shown on table 3.1. To account for both differences in the percentage of LC with particles and differences in the mean fluorescence associated with these cells, the product of the two parameters (\% association $\mathrm{x}$ mean fluorescence), termed index, was used routinely (fig. 3.10). Unlike the phagocytic index traditionally used in the assessment of phagocytosis by $\mathrm{M} \varnothing$ (e.g. reference [230]), index in this context cannot be translated into number of particles per LC. However, it is very useful in comparing samples obtained within one experiment as will become increasingly apparent throughout this thesis. ${ }^{2}$

\footnotetext{
2 Because the flow cytometer has to be calibrated for each experiment, mean fluorescence values diffe, depending on the settings used. Furthermore, even when the same settings are used consistently between sessions on the flow cytometer, drift of the laser beam with time prevents comparison of mean fluorescence values obtained in different sessions. This fact, not always appreciated even by FACS users (e.g., reference [63]), should be borne in mind by the reader whenever tempted to compare mean fluorescence or index values between different figures throughout this thesis. Percentage of positive cells, on the other hand, is independent of the calibration of the instrument.
} 
Table 3.1: Strain differences in the extent of uptake of zymosan by $L C$ after overnight incubation

\begin{tabular}{ccc}
$\begin{array}{c}\text { Mouse } \\
\text { strain }\end{array}$ & $\begin{array}{c}\text { \% cells } \\
\text { labelled }\end{array}$ & $\begin{array}{c}\text { Mean } \\
\text { fluorescence }\end{array}$ \\
\hline $\mathrm{C} 57 \mathrm{Bl} / 6$ & 97.8 & 637.20 \\
$\mathrm{C} 3 \mathrm{H} / \mathrm{HeJ}$ & 84.2 & 327.11 \\
$\mathrm{BALB} / \mathrm{c}$ & 67.6 & 250.47 \\
\hline
\end{tabular}

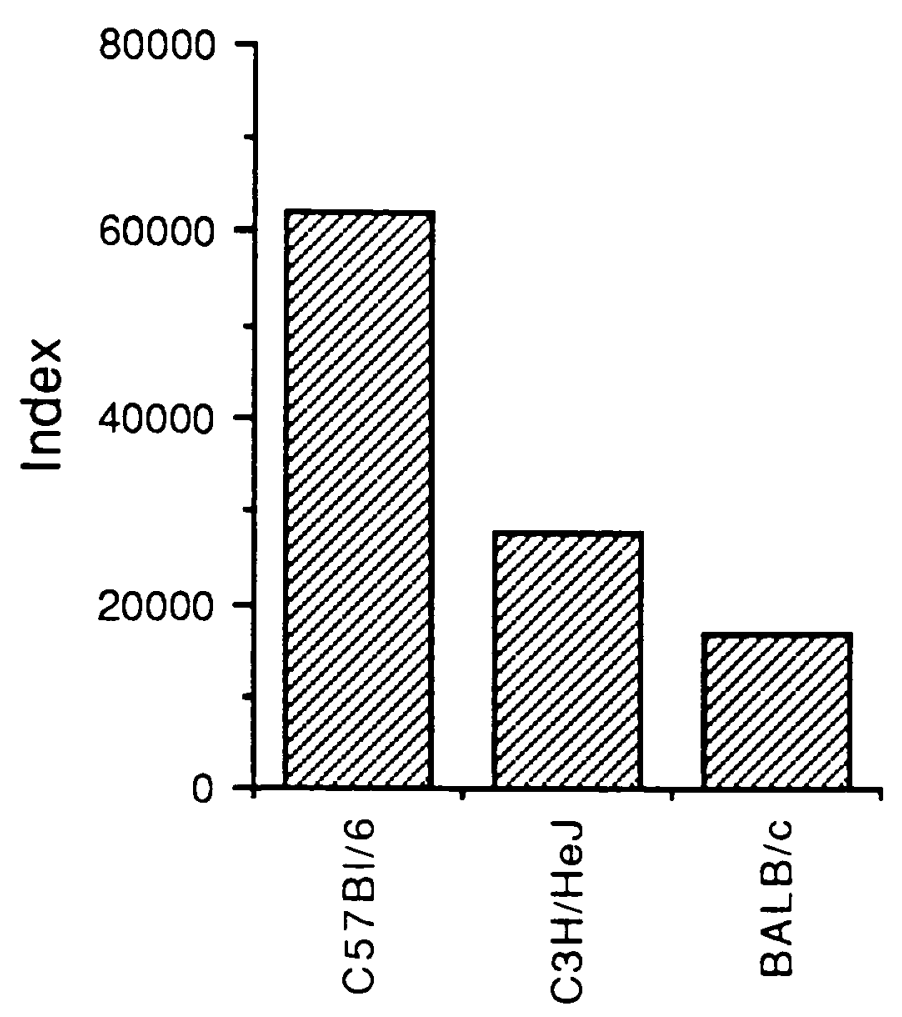

Fig. 3.10: Graphic representation of the data in table 3.1 as an index.

If index is used as a criterion instead of $\%$ association to analyse the data presented so far, it is confirmed that no particle shows such marked strain differences as zymosan with respect to extent of uptake. Zymosan indices were routinely $>3$-fold greater for $\mathrm{C} 57 \mathrm{Bl} / 6$ than for $\mathrm{BALB} / \mathrm{c} \mathrm{LC}$ in over 10 independent experiments. Variable, small differences were found for all other particles during long incubations and there was no true evidence for strain differences except, perhaps, for S. aureus where indices were around 1.6-fold greater for $\mathrm{C} 57 \mathrm{Bl} / 6$ than for $\mathrm{BALB} / \mathrm{C} \mathrm{LC}$ in two experiments. Note that no difference in the \% LC associated with S. aureus was observed (fig. 3.7a), highlighting the importance of analysing the mean FITC fluorescence of the cells. Strain differences in the extent of uptake by fresh LC during short (90 min) assays were far more variable and not consistent between experiments. This may be due to a variety of factors, primarily the time of culture chosen for the assay (see chapter 4). Overall, it appeared that C57Bl/6 LC had a greater phagocytic potential for all the particles tested but the extent to which they differed from $\mathrm{BALB} / \mathrm{c}$ LC was not investigated systematically. The discrepancy between this result 
and that obtained with long incubations where no differences were apparent suggests that strain differences in the extent of uptake after 90 min incubation may be due to differential rates of uptake rather than the overall size of the phagocytic compartment. The notable exception, once again, was zymosan where strain differences were very clear during the initial $0-24$ h of culture, with short as well as with long assays (see section 4.4). Strain differences in the phagocytosis of zymosan by LC are addressed further in the next chapters.

\section{5 - Comparison of Langerhans cells and macrophages}

The results presented so far clearly indicate that freshly isolated LC are phagocytic in vitro. However, it is also apparent that phagocytosis by LC is different from that by "professional phagocytes" such as macrophages. LC did not phagocytose opsonised SRBC via Fc receptors or aldehyde-fixed erythrocytes (see section 3.2) and LC did not bind C'SRBC (section 3.2) and did not take up colloidal carbon during an overnight incubation (not shown). In addition to qualitative differences, quantitative differences in phagocytosis between LC and M $\varnothing$ were apparent. Uptake by LC was far slower (fig. 3.6c) and more limited in extent than that by $\mathrm{M} \varnothing$. For example, LC routinely phagocytosed less than 8 particles of zymosan during a long incubation (fig. $3.11 \mathrm{a}, \mathrm{b}$; see also figs. $3.1 \mathrm{~h}, \mathrm{j}$ and $\mathrm{l}$ ) whereas resident peritoneal $\mathrm{M} \varnothing$ often ingested $\geq 20$ (fig. $3.11 \mathrm{c}, \mathrm{d}$ ). In addition, no strain differences in the uptake of zymosan by M $\varnothing$ were ever noted, in contrast to LC (data not shown; see table 5.2). Direct comparison of the average number of zymosan particles ingested (table 3.2) indicates that the phagocytic capacity of LC for zymosan is about three times lower than that of resident $\mathrm{M} \emptyset$. This difference is even greater when elicited $\mathrm{M} \emptyset$ are compared to LC. The flow cytometric indices obtained for thio-M $\varnothing$ were at least 5-fold greater than those for LC (fig. 3.12), mostly because of higher mean fluorescence associated with the cells rather than higher \% association with particles. In fact, part of the fluorescence associated with the $\mathrm{M} \emptyset$ in fig. 3.12 was off the top of the scale of the flow cytometer and the mean fluorescence was underestimated. 


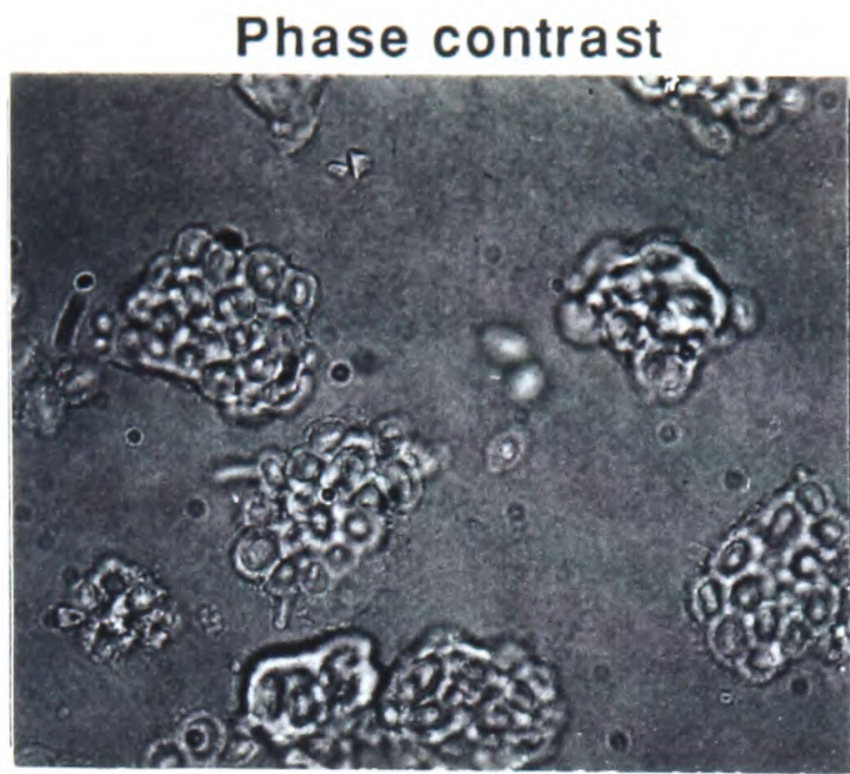

a

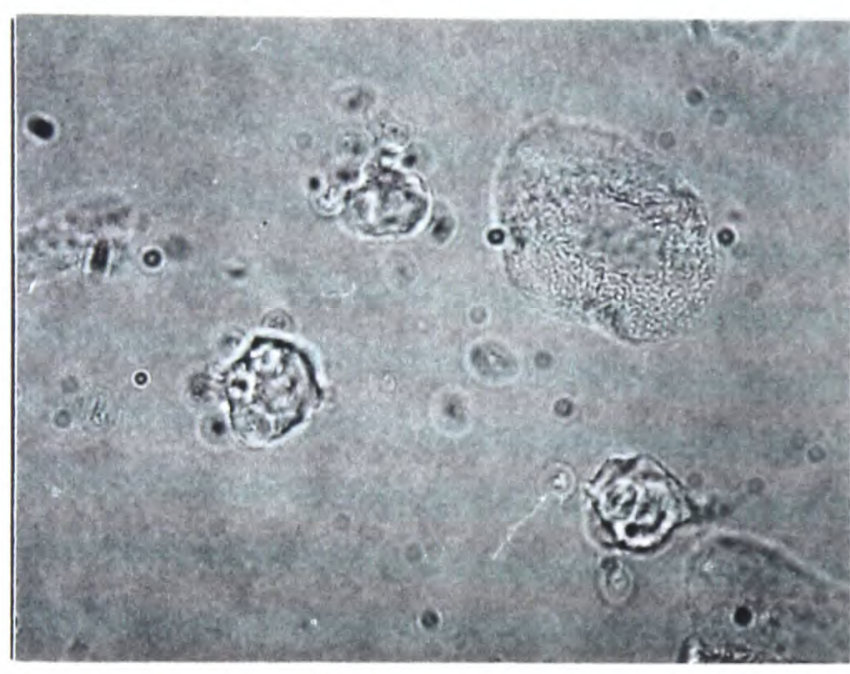

C

\section{Fluorescence}

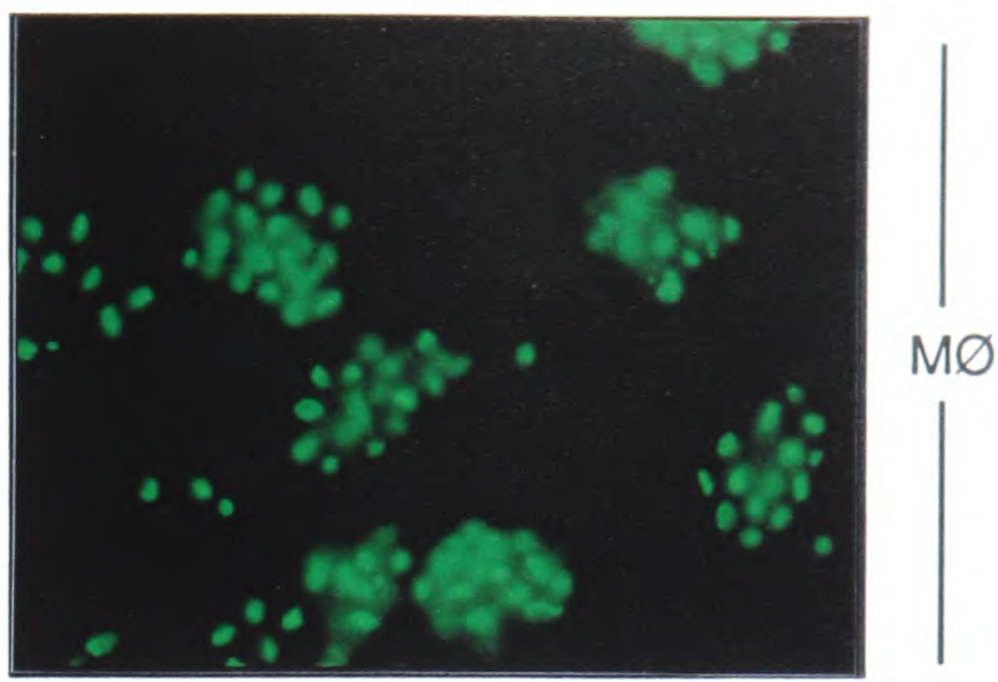

b

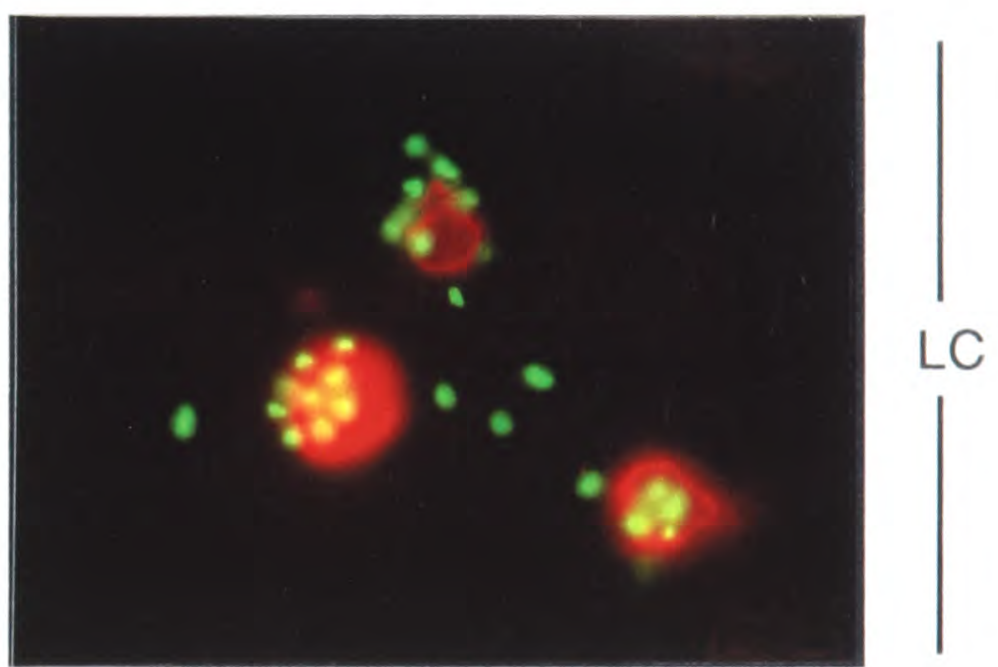

d

Fig. 3.11: Comparison of the phagocytic potential of $\mathrm{LC}$ and resident $\mathrm{M} \emptyset$. Resident peritoneal MØ (a,b) or fresh EC (c,d) were incubated with FITC-zymosan for $16 \mathrm{~h}$. The low-density fraction of EC was stained for MHC class II antigens with Texas Red to identify LC (d). All photographs were taken at $\mathrm{x} 1000$. The right-hand photograph in each pair shows the fluorescence image for the phase contrast field shown on the left. Filters: b: green; $\mathbf{d}$ : red and green (double exposure).

Table 3.2: Average number of zymosan particles internalised per phagocytosing cell

\begin{tabular}{cccc}
\hline & Mean & SD & $\mathrm{n}$ \\
\cline { 2 - 4 } Resident MØ & 14.1 & 3.9 & 51 \\
Fresh LC & 5.2 & 3.1 & 40 \\
\hline
\end{tabular}

Data from the experiment shown in fig. 3.11. 


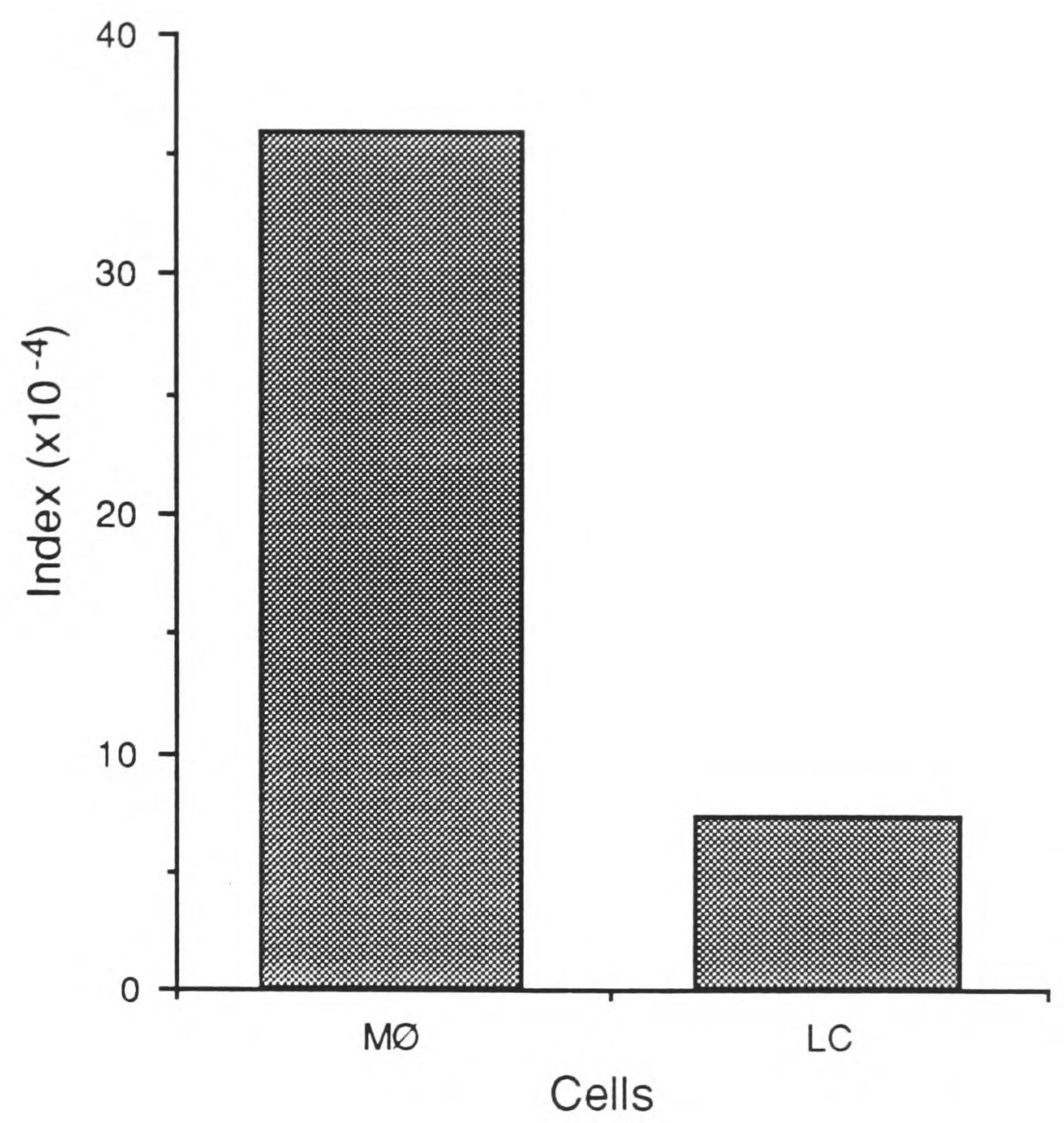

Fig. 3.12: Comparison of the phagocytic potential of $\mathrm{LC}$ and elicited MØ. Fresh $\mathrm{C} 57 \mathrm{Bl} / 6 \mathrm{EC}$ or thio-M $\varnothing$ were pulsed for $16 \mathrm{~h}$ with FITC-zymosan in polypropylene tubes. Double colour FACS analysis of LC was as before. M $\varnothing$ were analysed by single colour fluorescence. The index for $\mathrm{M} \varnothing$ may be underestimated (see text).

These quantitative differences between LC and M $\varnothing$ were not restricted to zymosan and were observed for all particles tested (not shown). Thus, LC should not be regarded as macrophages of the epidermis but, rather, the comparatively limited capacity of LC to phagocytose is suggestive of their primary function in antigen presentation instead of immune clearance. 


\section{6 - Summary}

This chapter has shown that freshly isolated epidermal Langerhans cells can phagocytose latex microspheres ranging from $0.5-3.5 \mu \mathrm{m}$ diameter, zymosan and $S$. cerevisiae, $C$. parvum and S. aureus (section 3.2 and 3.3). However, particles were internalised more slowly and to a lesser extent by LC than M $\varnothing$ (section 3.5). In addition, unlike MØ, LC did not appear to be capable of phagocytosing erythrocytes via FcR, or to attach them via CR3 (fig. 3.3), although at least the latter may synergise with other receptors in the uptake of certain particles (notably, C. parvum - see fig. 3.7), as noted for other cell types [215]. A further difference in the phagocytic capacity of LC and M $\varnothing$ was also evident from the observation that fresh LC were unable to take up colloidal carbon (not shown) and aldehyde-fixed erythrocytes (fig. 3.4), suggesting that qualitatively distinct receptors are present on the two cells. Whilst most of the work presented in this chapter made use of $\mathrm{C} 57 \mathrm{Bl} / 6 \mathrm{LC}$, important differences were found with respect to the extent of uptake of zymosan when other strains were compared (fig. 3.9). During long incubations strain variations in the uptake of particles other than zymosan were small although it is possible that $\mathrm{C} 57 \mathrm{Bl} / 6 \mathrm{LC}$ take up more $S$. aureus than $\mathrm{BALB} / \mathrm{c}$ (see text, section 3.4). In contrast, when shorter (90 min) incubations were used, strain differences for particles other than zymosan were far more variable and, overall, suggested that $\mathrm{C} 57 \mathrm{Bl} / 6 \mathrm{LC}$ were more phagocytic. A summary of the results presented in this chapter for different particles is given in table 3.3. 
Table 3.3: Summary of observations on the phagocytic potential of fresh C57Bl/6 LC

Method of assessment

\begin{tabular}{|c|c|c|c|c|}
\hline & & & & \\
\hline Particle & Phagocytosis & $\begin{array}{l}\text { Light/ UV } \\
\text { microscopy }\end{array}$ & $\begin{array}{c}\text { Electron } \\
\text { microscopy }\end{array}$ & $\begin{array}{c}\text { Flow } \\
\text { cytometry }\end{array}$ \\
\hline Zymosan & +++ & $\sqrt{ }$ & $\sqrt{ }$ & $\sqrt{ }$ \\
\hline Zymosan + NMS & +++ & $\sqrt{ }$ & & $\sqrt{ }$ \\
\hline S. cerevisiae & +++ & $\sqrt{ }$ & & \\
\hline $0.5 \mu \mathrm{m}$ latex beads ${ }^{*}$ & +++ & $\sqrt{ }$ & $\sqrt{ }$ & $\sqrt{ }$ \\
\hline $1 \mu \mathrm{m}$ latex beads ${ }^{*}$ & ++ & $\sqrt{ }$ & $\sqrt{ }$ & $\sqrt{ }$ \\
\hline $3 \mu \mathrm{m}$ latex beads $*$ & + & $\sqrt{ }$ & $\sqrt{ }$ & $\sqrt{ }$ \\
\hline C. parvum & ++ & $\sqrt{ }$ & & $\sqrt{ }$ \\
\hline C. parvum + NMS & +++ & $\sqrt{ }$ & & $\sqrt{ }$ \\
\hline S. aureus & +++ & $\sqrt{ }$ & & $\sqrt{ }$ \\
\hline S. aureus + NMS & +++ & $\sqrt{ }$ & & $\sqrt{ }$ \\
\hline Colloidal carbon & - & $\sqrt{ }$ & & \\
\hline$S R B C \pm I g G$ & - & $\sqrt{ }$ & & \\
\hline $\mathrm{SRBC} \pm \operatorname{Ig} M$ & - & $\sqrt{ }$ & & \\
\hline$S R B C \pm C^{\prime}$ & - & $\sqrt{ }$ & & \\
\hline Aldehyde-fixed SRBC & - & $\sqrt{ }$ & & \\
\hline FITC-SRBC & - & $\sqrt{ }$ & & \\
\hline $\begin{array}{l}\text { Autologous RBC } \pm \\
\text { autologous serum } \\
\end{array}$ & - & $\sqrt{ }$ & & \\
\hline
\end{tabular}

Compilation of results from different experiments using long (12-18h) incubations and saturating doses of particles. Uptake was assessed by microscopy in all cases and was further quantified by flow cytometry where appropriate. +++ , $>80 \%$ LC phagocytose,,$++ 30-80 \%$ LC phagocytose,,$+ 10-30 \%$ LC phagocytose,,$-<1 \%$ LC phagocytose.

Because of differences in mass comparisons of uptake between latex beads of different sizes are difficult (see section 3.3); the values in this table reflect those from fig. 3.9. 


\section{Chapter 4}

\section{Cultured Langerhans cells and splenic dendritic cells have little phagocytic capacity}

\section{1 - Introduction}

4.2 - Cultured LC have little phagocytic capacity

4.3 - Pulsed LC retain and degrade particulate antigen during culture.

4.4 - Strain differences in the kinetics of down regulation of zymosan uptake

4.5 - Splenic DC resemble cultured LC in their phagocytic ability.

4.6 - Summary 


\section{1 - Introduction}

Maturation of Langerhans cells in culture into cells virtually indistinguishable from lymphoid dendritic cells is a well established phenomenon [125]. A striking feature of this process is the loss of ability to present native antigens whilst the ability to present immunogenic peptides and to act as stimulators in a mixed lymphocyte reaction is markedly increased. The reasons for this loss of native antigen presentation could be several-fold and there is evidence for changes both at the level of antigen processing per se and the formation of immunogenic peptide/MHC complexes. The loss of acidic vesicles where processing is likely to occur, has been observed during maturation of both human and murine LC in culture [133]. On the other hand, Puré and colleagues [35] concluded that differences in the presenting ability of these cells were likely to be due to the fact that freshly isolated but not cultured LC actively synthesised MHC class II molecules and invariant chain and not due to differences in the ability of the two cell types to pinocytose antigens. However, the internalisation of antigens by endocytosis or phagocytosis is commonly thought to be a prerequisite for processing and the relative ability of different cell types to perform these functions may determine the extent of presentation [29]. Remarkably, changes in the ability of LC to internalise antigens during maturation have remained largely unexplored. Indirect evidence for the capacity of LC and VC to acquire antigens (see chapter 1) contrast sharply with the observations that lymphoid DC and veiled cells in vitro are non-phagocytic and poorly endocytic $[55,89]$ and suggest that any ability Langerhans cells may have to capture antigens is lost during maturation. Our finding that freshly isolated LC are phagocytic in vitro (chapter 3) provided an ideal model system to investigate this hypothesis.

In this chapter, the capacity of cultured epidermal LC to phagocytose antigens is evaluated using the techniques introduced in chapter 1 and is compared to that of fresh LC. In addition, in the light of recent reports that some splenic DC may undergo a maturational 
process similar to that of LC during short-term culture $[118,126,143]$, the capacity of these lymphoid DC to phagocytose is re-evaluated and compared to that of LC. We report that LC and freshly isolated splenic DC down regulate their phagocytic ability in culture and come to resemble mature splenic DC in having only a limited capacity to internalise particulate antigens. 


\section{2 - Cultured LC have little phagocytic capacity}

Using a similar procedure to that employed for the analysis of uptake by fresh LC (fig. 3.1), EC that had been cultured for three days were incubated with fluorescent particles for $18 \mathrm{~h}$ before harvesting the non-adherent fraction and enriching for LC over dense BSA columns. Cultured LC, plated on PLL-coated coverslips, had the typical morphology of dendritic cells such as large veils and processes that stained intensely for class II antigens (fig. 4.1 a,d,g) and their appearance contrasted greatly with the more "hairy" appearance of fresh LC (fig. 3.1i,j). Cultured LC showed little evidence of uptake of FITC-zymosan (fig. 4.1 a-c; compare with fig. 3.1 g,h,j), FITC-C. parvum (fig. 4.1d,e; compare with fig. 3.1a,b) or FITC-S. aureus (fig. 4.1g,h,i; compare with fig. 3.1e,f,i) and neither did they ingest $0.5,1$ or $3 \mu \mathrm{m}$ latex beads (not shown). This marked lack of phagocytic ability was also evident from phase contrast and differential interference contrast microscopy. To act as positive controls for internalisation, fresh LC (from the same mice) were included in each experiment and the cells shown in fig. 4.1 were, in fact, part of the experiment described in fig. 3.1 and can be compared directly. Ia- cells in the cultured EC population that showed active uptake (fig. 4.1f,i) acted as additional internal positive controls. The lack of uptake by cultured LC was not due to decreased viability since they excluded trypan blue (not shown) and actively spread on PLL-coated coverslips (fig. 4a,d,g) whereas contaminating dead keratinocytes did not. Furthermore, the culture system employed here has been used extensively by other groups and shown to maintain the viability of LC in the absence of exogenous factors [120, 137-140]. Interestingly, cultured LC were not totally devoid of phagocytic activity since, occasionally, $\mathrm{Ia}^{+}$cells associated with particles were seen (fig. $4.1 \mathrm{c}$, cell on the top left corner). 


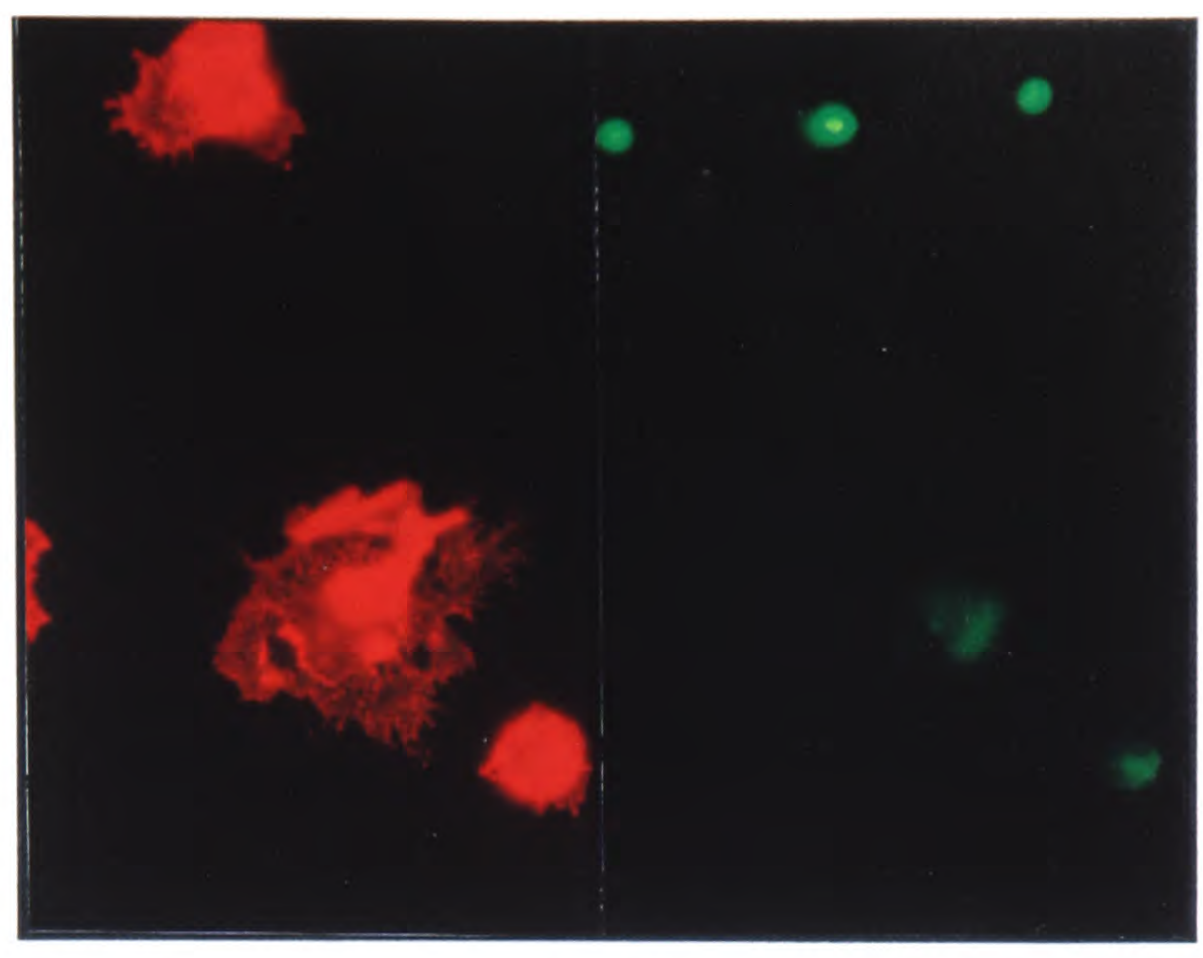

a

b
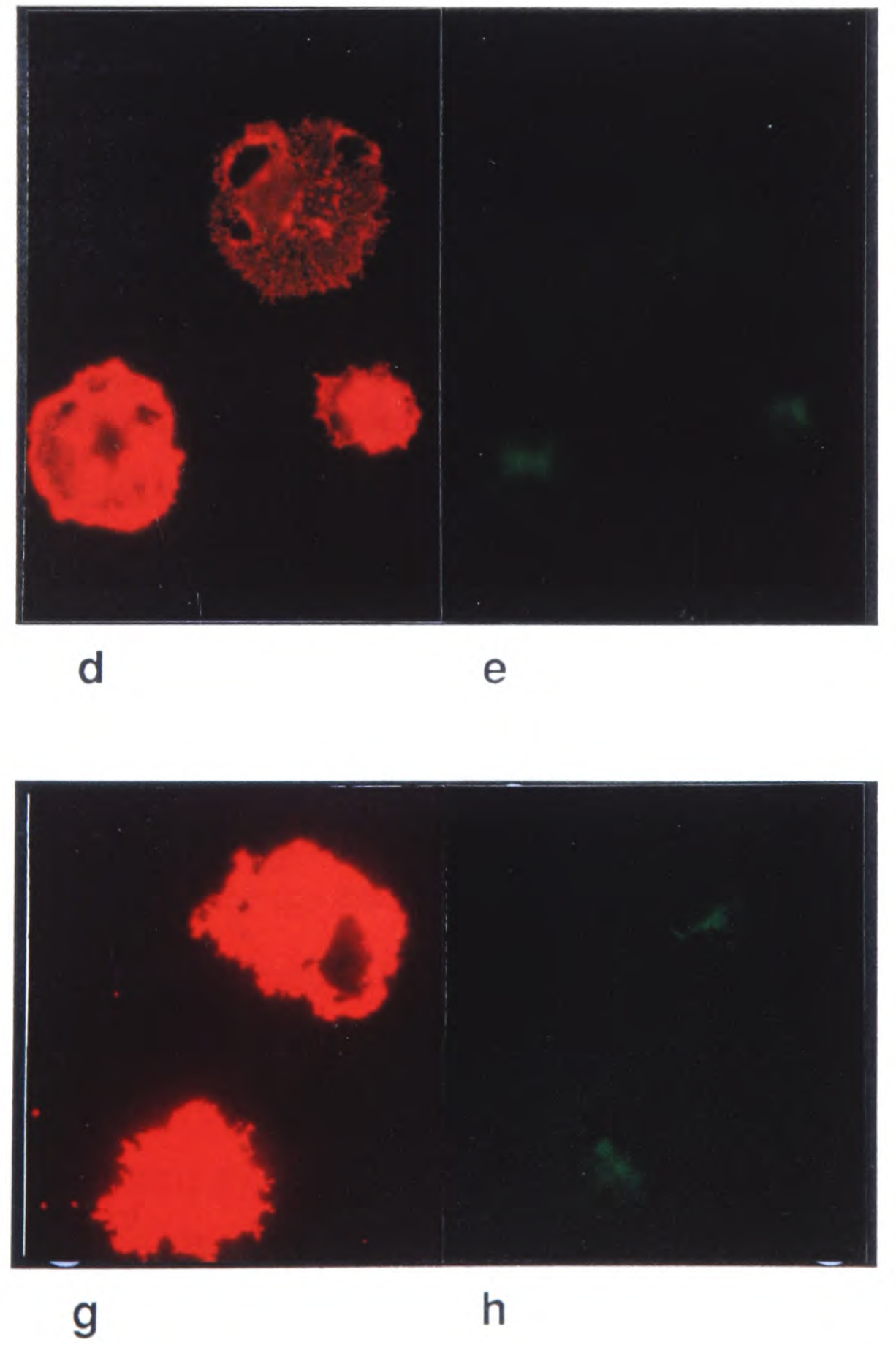
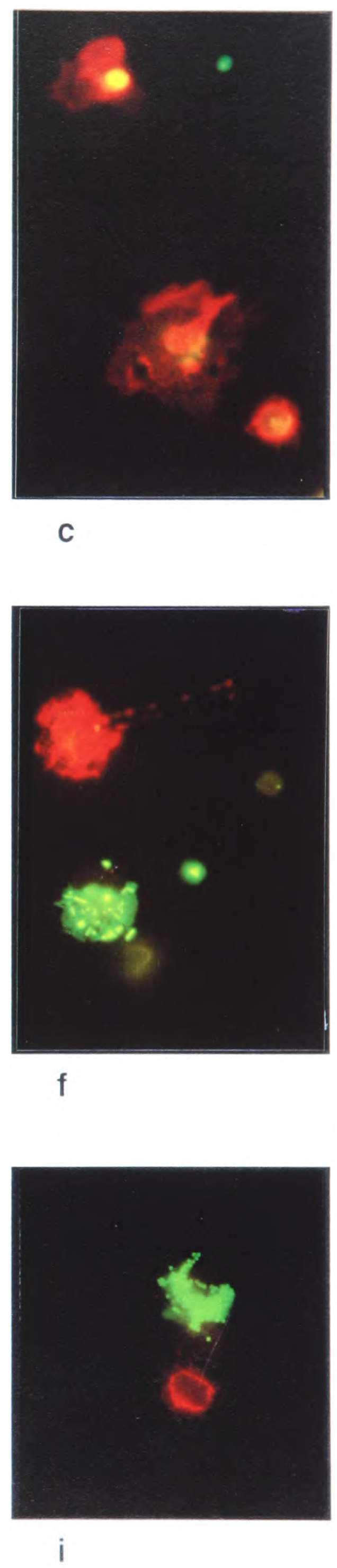
Fig. 4.1: Cultured LC are poorly phagocytic in vitro. Bulk EC from the experiment shown in fig. $3.1 \mathrm{a}, \mathrm{b}, \mathrm{e}-\mathrm{j}$, were pulsed for $18 \mathrm{~h}$ with the indicated particles after culturing for 72h. Low density cells were stained for Ia with Texas Red. a-c) FITC-zymosan, d-f) FITC-C. parvum, g-i) FlTC-S. aureus. c), f) and i) are double exposures (red and green) of the same field and appear yellow where the two colours are superimposed. All other photographs are paired, red (left) and green (right), fluorescence images of the same field. c) is the same field as a) and b) and demonstrates that the cell on the top left corner of is associated with one zymosan particle. All photographs were taken at an initial magnification of $\times 1000$.

It is clear from this experiment (representative of three other similar experiments) that cultured LC have greatly lost the ability to acquire particulate antigens for presentation. To quantify this loss, we made use of the flow cytometric method previously employed for the analysis of uptake by fresh LC (section 3.3). An example of this type of analysis for the uptake of $S$. aureus by fresh and cultured LC is given in fig. 4.2 a-d. Staining of bulk EC with an anti-MHC class II antibody in conjunction with PE allowed clear identification of the LC component and showed less association of particles with cultured LC (fig. 4.2b) than with fresh LC (fig. 4.2a); the increase in the relative proportion of MHC class II-positive cells in cultured versus fresh EC (from 3.4 to $6.1 \%$ in fig. $4.2 \mathrm{a}, \mathrm{b}$ ) is due to adherence of keratinocytes during culture. To obtain more accurate data on LC, $\mathrm{Ia}^{+}$cells were gated to the exclusion of MHC class II-negative keratinocytes and, possibly, small numbers of $\gamma / \delta \mathrm{T}$ cells, and their profile was displayed as a double histogram where the solid line represents fresh LC and the dotted line represents cultured LC (fig. 4.2c). The reader is referred to fig. $3.5^{1}$ and to chapter 2 for further details.

The markedly decreased phagocytic capacity of cultured versus fresh LC for $S$. aureus is evident from the profile shown in fig. 4.2c. Cultured LC not only have fewer particles associated with them $(20.7 \%$ of cultured LC with particles versus $95.5 \%$ of fresh

\footnotetext{
1 The data shown for fresh LC in fig. 4.2a,c,d are those presented in the previous chapter (fig. 3.5) in order to facilitate comparison by the reader.
} 
LC) but also have a fluorescence peak one log decade lower than that of fresh LC indicating association with fewer particles per phagocytic cell. As before (fig. 3.10), these two parameters (percentage of LC associated with particles and mean fluorescence) can be combined into an index (fig. 4.2d) to provide a visual representation of the down regulation taking place. Examples of similar analyses for $1 \mu \mathrm{m}$ latex beads and zymosan are shown in figs. $4.2 \mathrm{e}, \mathrm{f}$ and $\mathrm{g}, \mathrm{h}$, respectively. Note that down regulation of uptake has occurred by 2 days of culture (fig $4.2 \mathrm{f}, \mathrm{h}$ ) when the cells are already thought to be functionally mature [120]. In fact, comparison of zymosan uptake by LC cultured for 0,1 and 2 days reveals that down regulation is virtually complete by $24 \mathrm{~h}$ of culture (data not shown and table 4.2 ). The analysis of uptake $3 \mu \mathrm{m}$ latex (not shown) beads gave similar results to that of $1 \mu \mathrm{m}$ beads (fig. $4.2 \mathrm{e}, \mathrm{f}$ ) but the differences between cultured and fresh cells were much less marked for $0.5 \mu \mathrm{m}$ beads, particularly at the level of percentage of LC associated with beads (data not shown and table 4.3). This may reflect the fact that particles of this size can be partly taken up by pinocytosis (section 1.3 ). The residual uptake at $72 \mathrm{~h}$ of culture was a feature of all particles tested and correlates well with the microscopical observations noted above (see fig. $4.1 \mathrm{c}$ ). The extent of this residual uptake varied between experiments and between particles in the range of 5-30\% phagocytic LC, reflecting variability in the extent of maturation. However, in over 5 experiments using long incubations, it was always lower than that observed with fresh LC and was strain independent in the two strains analysed (C57B1/6 and BALB/c). 


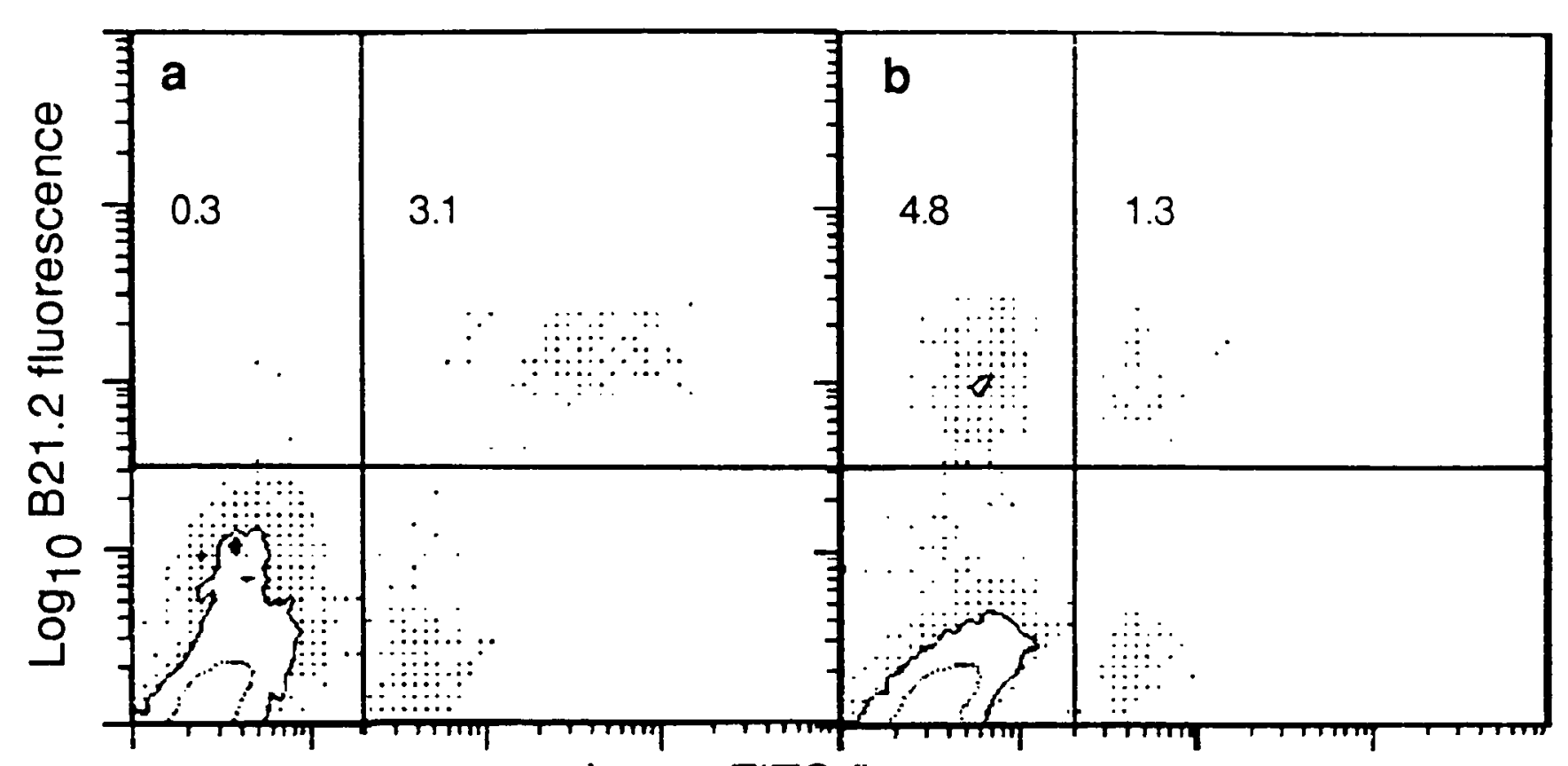

$\log _{10} \mathrm{FITC}$ fluorescence
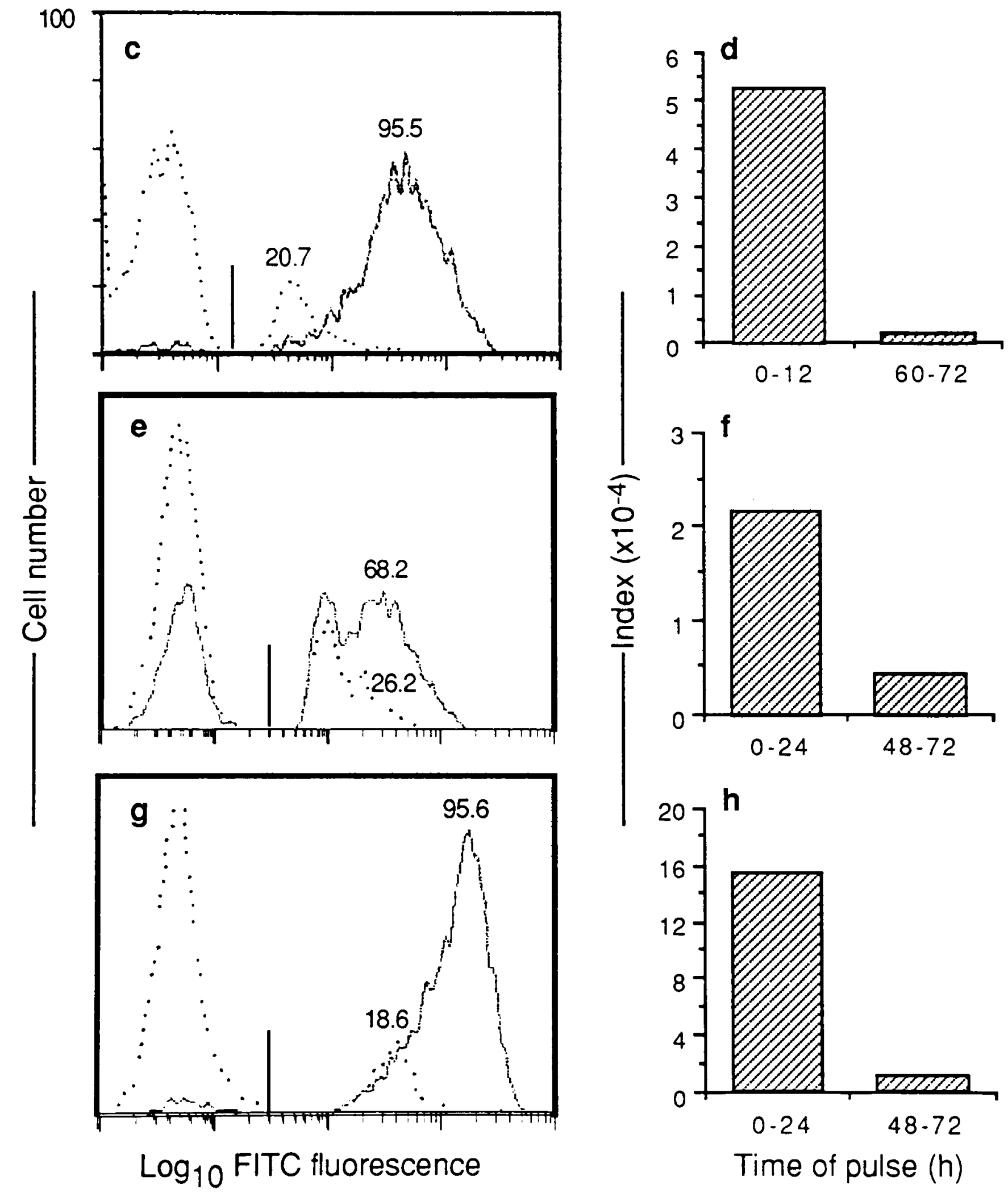
Fig. 4.2: LC down regulate the ability to phagocytose antigens in vitro. Bulk EC from $\mathrm{C} 57 \mathrm{Bl} / 6$ mice were pulsed with particles before and after culture and analysed by FACS as described in the methods. a) double fluorescence plot of whole EC pulsed with FITC-S. aureus for $12 \mathrm{~h}$ immediately after isolation showing B21.2 staining (Ia) on the vertical axis and FITC ( $S$. aureus) on the horizontal axis; b) same cells pulsed at $60 \mathrm{~h}$ of culture. Numbers in a) and b) indicate the percentage of cells in that quadrant. c) FITC fluorescence of gated B21.2+ cells from a) and b); solid line represents fresh LC dotted line represents cultured LC; numbers represent the \% FITC-positive LC for each population. d) visual representation of the data in c) using an index (the product of $\%$ FITC-positive LC by their mean FITC fluorescence). e,f) analysis of uptake of $1 \mu \mathrm{m}$ fluorescent latex beads as in c,d) after a $24 \mathrm{~h}$ pulse at $0 \mathrm{~h}$ or $48 \mathrm{~h}$ of culture. $\mathbf{g , h}$ ) as for e) and f) using FITC-zymosan. e-h are from the same experiment; a-d are from a separate experiment and a) is also shown in fig. 3.5. Note that index values cannot be compared between particles because of intrinsic differences in mean fluorescence.

Langerhans cells migrate out of skin explants in organ culture and, with time, acquire functional properties similar to those of cultured LC [104]. However, they are a more heterogeneous population than the latter and contain cells with phenotypic characteristics of fresh LC (expression of Fc receptors, F4/80), presumably because of continuous emigration of immature cells from the explant. It is a working hypothesis that these migratory cells have entered skin lymphatics, falling to the bottom of the culture dish because the vessels are severed. As such, these migratory LC may represent the functional equivalent of veiled cells isolated from lymph $[89,90]$. Being not quite as mature as cultured LC isolated in the conventional way, they may be expected to be more phagocytic than the latter. To address this issue, the two cell types were pulsed with FITC-zymosan and $\mathrm{Ia}^{+}$cells were examined by FACS for association with the particles. The results, presented in table 4.1, indicate that migratory $\mathrm{Ia}^{+}$cells from ear skin cultured for 3 days are indeed more phagocytic for zymosan than LC from epidermal cell suspensions cultured for the same time, as reflected both in a higher \% of cells with zymosan and a higher mean fluorescence. The fact that about $40-60 \%$ of the migratory LC are phagocytic for zymosan 
was confirmed by microscopy using unconjugated zymosan in four independent experiments and this, most likely, reflects their intermediate phenotype, consisting of a mixture of fresh, mature and maturing LC.

Table 4.1: Comparison of the phagocytic potential of cultured LC and migratory cells

\begin{tabular}{cccc}
\hline Dendritic leukocyte & \% association & Mean fluorescence & Index \\
\hline d3 cultured LC & 19.9 & 405.59 & 8071.24 \\
d3 migratory cells & 54.5 & 786.09 & 42841.91 \\
\hline
\end{tabular}

Ears from C57B1/6 mice were used to prepare EC suspensions or were floated on medium to obtain migratory cells. After 3 day culture, migratory cells were collected and plated as for the cultured EC suspensions. Dishes were pulsed for 90 min with a saturating dose of FITC-zymosan and $\mathrm{Ia}^{+}$cells were analysed by FACS.

We conclude from these experiments that the phagocytic activity of LC is markedly reduced after maturation in culture. Cultured cells have not totally lost the capacity to phagocytose antigens but this is likely to reflect heterogeneity in the maturational state of the LC population, similar to that observed in experiments looking at loss of antigen processing (N. Romani, personal communication). Migratory LC, the putative in vitro equivalent of veiled cells, are also phagocytic for zymosan and for latex (not shown) and their phagocytic potential is intermediate between that of fresh and cultured LC. The rapid maturation of non-lymphoid LC into poorly phagocytic cells is likely to explain the observation that mature lymphoid DC are non-phagocytic [55].

\section{3 - Pulsed LC retain and degrade particulate antigen during culture.}

In order to participate in the generation of immune responses against particulate antigens such as bacteria or yeasts, LC must degrade internalised particles to peptides capable of associating with newly-synthesised MHC class II molecules. Evidence for degradation of zymosan is apparent from fig. 3.2e and, possibly, from fig. 3.6c. Given that fresh LC are phagocytic whereas mature LC are only poorly so, it was of interest to determine whether 1) fresh LC pulsed with particles are capable of retaining antigen during 
maturation (i.e., do not lose particles by regurgitation), and 2) partial or complete degradation of internalised particles occurs during this period. Ideally, these questions would best be answered using antigen-presentation assays with antigen-specific $\mathrm{T}$ cell clones or hybridomas responding to particle-pulsed LC. Unfortunately, latex and zymosan are non-immunogenic and $\mathrm{T}$ cells specific for the bacteria used here were also not available for this study. Attempts to prime lymph node $T$ cells against yeast extract, with the view of studying presentation by LC pulsed with $S$. cerevisiae, were unsuccessful (not shown). Therefore, use was made of the flow cytometry assay, once again, to provide an indirect answer. Fresh EC from C57Bl/6 mice were pulsed with zymosan and harvested at 24,48 or $72 \mathrm{~h}$ of culture. A clear decrease in index with time was observed, suggesting that LC were degrading internalised particles (table 4.2). The interpretation is complicated by the fact that zymosan was not removed after the initial $24 \mathrm{~h}$ pulse and continuing phagocytosis of free particles might mask regurgitation and loss of previously acquired ones. However, parallel cultures showed that the cells down regulated their phagocytic ability as usual (compare index of LC pulsed from $0-24 \mathrm{~h}, 24-48 \mathrm{~h}$ and $48-72 \mathrm{~h}$ ) so total loss of ingested particles is unlikely to have been compensated by fresh phagocytosis.

Table 4.2: Evidence that $L C$ pulsed with zymosan on day 0 retain particles during culture

\begin{tabular}{cccc}
\hline & \multicolumn{3}{c}{ Time of pulsing (h) } \\
\cline { 2 - 4 } Time of fixation $(\mathrm{h})$ & 0 & 24 & 48 \\
\hline 24 & 28018 & ---- & ---- \\
48 & 14464 & 2967 & $-\cdots$ \\
72 & 10680 & $\mathrm{ND}$ & 1510 \\
\hline
\end{tabular}

C57Bl/6 EC were pulsed with a saturating dose of FITC-zymosan at the culture times indicated and were harvested and fixed 1-3 days thereafter. Data is shown as the index obtained after FACS analysis. Note that free zymosan was not removed following any of the pulses (see text).

A simplified version of the experiment was repeated where zymosan was removed after the initial $16 \mathrm{~h}$ pulse and the cells were recultured for a further 3 days (fig. 4.5). 
Because zymosan pellets with the cells, it cannot easily be washed off non-adherent EC.

To separate free particles from cells, the latter were fractionated over dense BSA and recultured with keratinocytes from the high-density BSA fraction of an unpulsed culture to act as a source of GM-CSF and other cytokines. Retention of particles by the recultured cells was manifest although the percentage of LC associated with zymosan decreased from 93.6 to $69.1 \%$, either because of complete particle degradation by $24.5 \%$ of cells or because of regurgitation. The mean fluorescence of recultured LC also decreased (notice shift in peak B compared to A) as would be expected from partial degradation. Control cells, pulsed for $16 \mathrm{~h}$ on day 3 , showed the expected down regulation of uptake (compare profile $\mathrm{C}$ with $\mathrm{A})$.

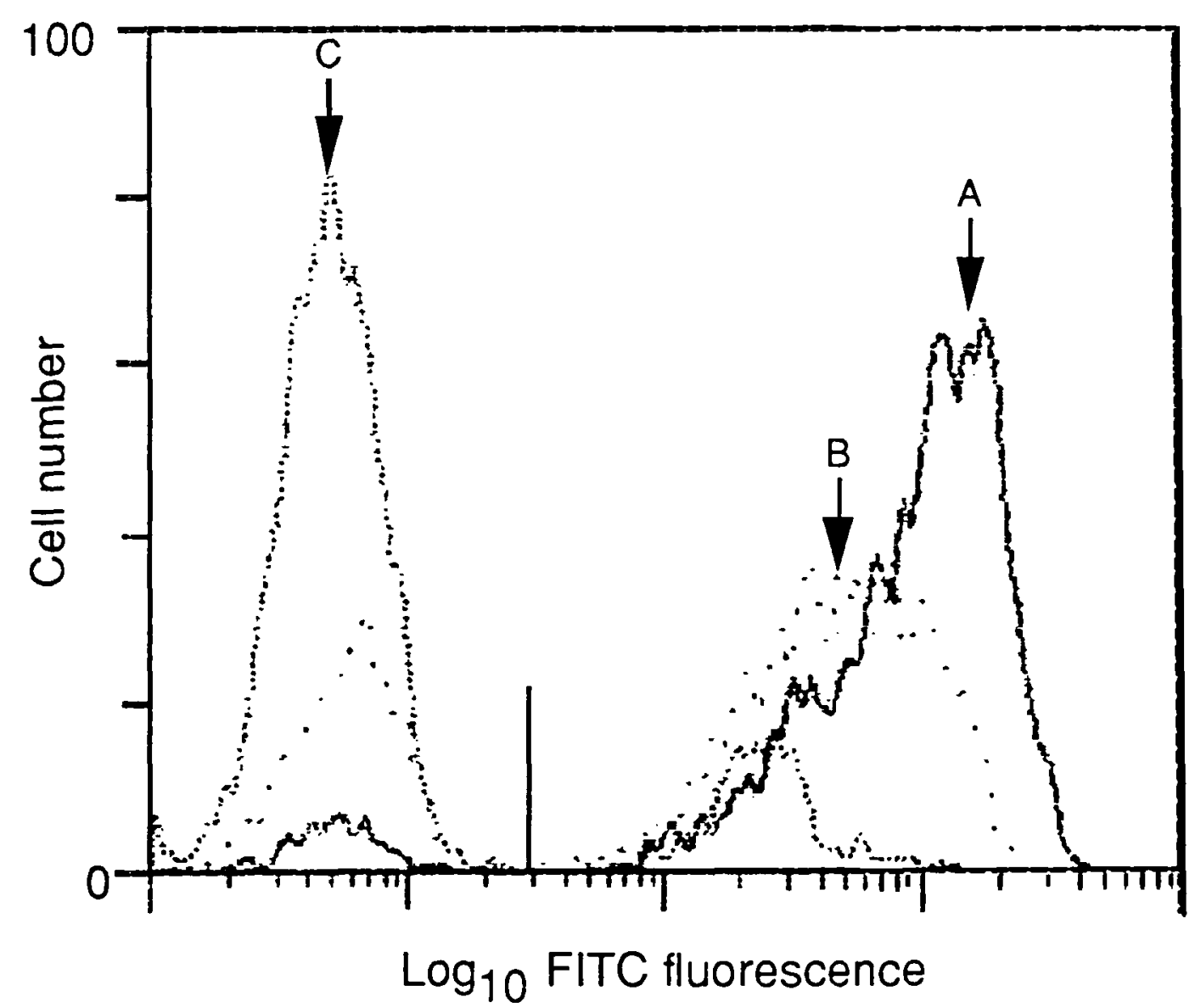

Fig. 4.3: Zymosan persists inside cells cultured for 4 days. EC were pulsed with FITCzymosan from $0-16 \mathrm{~h}$ and some were fixed for analysis (A). The remainder was separated over dense BSA to remove free particles and the low density pulsed EC were co-cultured with high density EC from a parallel unpulsed culture in conditioned medium and fixed at 
$88 \mathrm{~h}$ (B). A control culture, separated and reconstituted in the same way, was pulsed from $72-88 \mathrm{~h}(\mathrm{C})$. The analysis of LC was performed as described in the methods. \% uptake: 0 16h pulsed LC, 93.6, 0-16h pulsed LC recultured, 69.1, 72-88h pulsed LC, 16.3.

The tentative conclusion from these two experiments is that freshly-pulsed LC are capable of retaining particulate antigens whilst partly degrading them, conceivably generating immunogenic peptides. It is worth mentioning in this context that, in the course of this work, it was reported that LC can present peptides from whole Leishmania major to specific T cells [231], the first time LC have been shown to present particulate antigens to T cells.

\section{4 - Strain differences in the kinetics of down regulation of zymosan uptake}

Mouse strain differences in the extent of zymosan phagocytosis by LC were reported in section 3.3. During the course of long incubations, C57Bl/6 LC consistently had an index more than 3-fold higher than that of BALB/c LC. Using short incubations (90 min) at different times of culture, striking differences between the two strains were observed in the kinetics with which the phagocytic activity decreased during maturation (fig. 4.4a). C57B1/6 LC exhibited a highly reproducible (6 out of 7 experiments) rise in their phagocytic index for zymosan from 0 to $6 \mathrm{~h}$ of culture followed by a decline (fig. $4.4 \mathrm{a}$, filled squares), whereas this initial increase did not occur in LC from BALB/c mice (fig. $4.4 \mathrm{a}$, open squares). In contrast, the same $\mathrm{C} 57 \mathrm{Bl} / 6$ cells incubated with $1 \mu \mathrm{m}$ latex beads do not show this increase (fig. $4.4 \mathrm{~b}$, filled squares), demonstrating that it is not due to a generalised difference in the phagocytic ability of the two strains. Although, in this experiment, $\mathrm{C} 57 \mathrm{~B} 1 / 6 \mathrm{LC}$ also appear to be more phagocytic than BALB/C LC for latex beads at $6 \mathrm{~h}$ (fig. $4.4 \mathrm{~b}$ ), in two other experiments uptake was down regulated much more rapidly and the index values at $6 \mathrm{~h}$ were similar to those at $24 \mathrm{~h}$. The differential kinetics of zymosan uptake in the two strains were reflected by similar differences in binding at $4^{\circ} \mathrm{C}$ (fig. $4.4 \mathrm{a}$, circles), strongly suggesting that they are due to differential expression of 
specific receptors for zymosan in the two strains. ${ }^{2}$ The zymosan-specific rise in phagocytic activity for $\mathrm{C} 57 \mathrm{Bl} / 6 \mathrm{LC}$ was also observed in a single time course experiment performed at Washington University, U.S.A., using mice from a local supplier, demonstrating that it is not an artefact from the animals maintained at our facilities. This key observation forms the basis of the investigation into receptors for zymosan in Langerhans cells that is reported in chapter 5 .

a

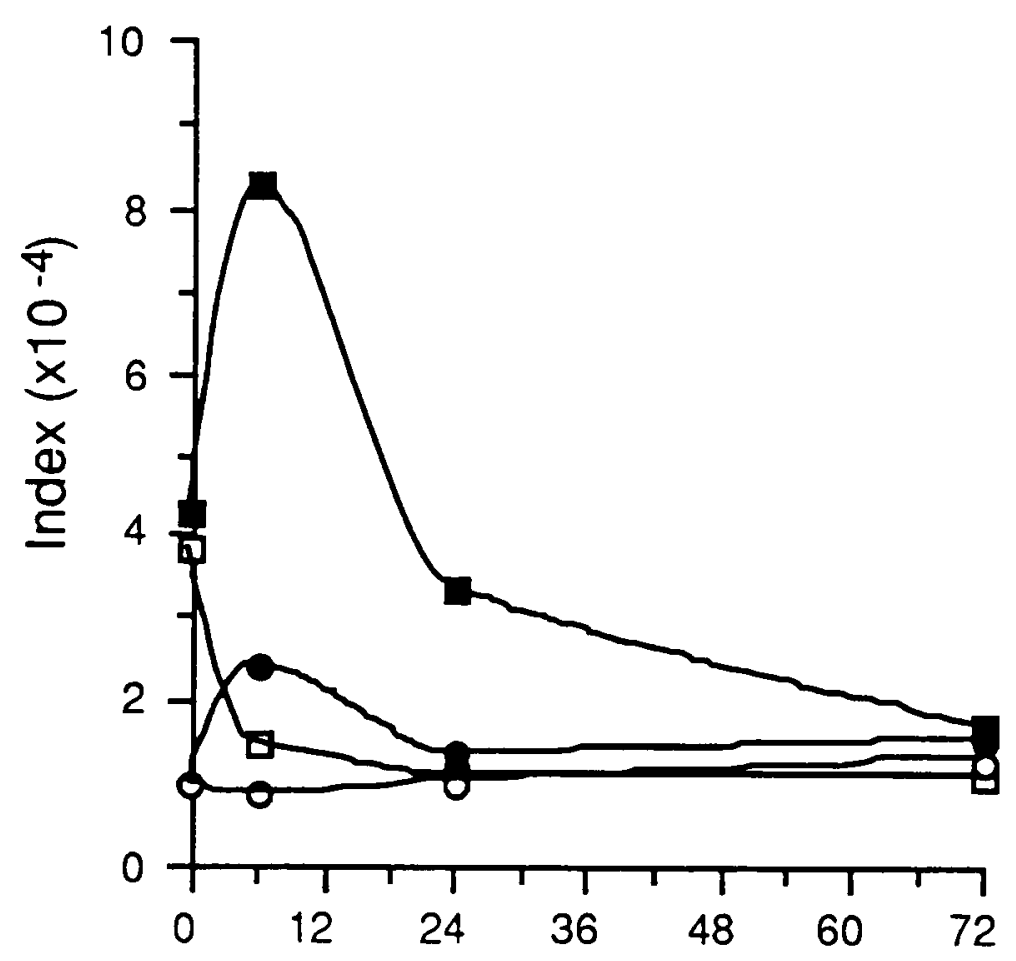

b

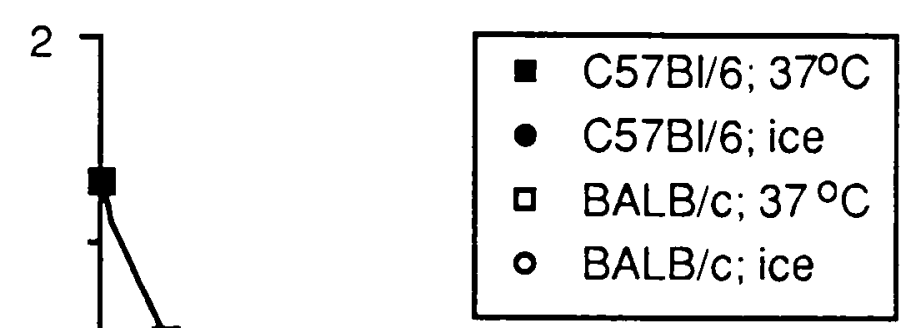

0

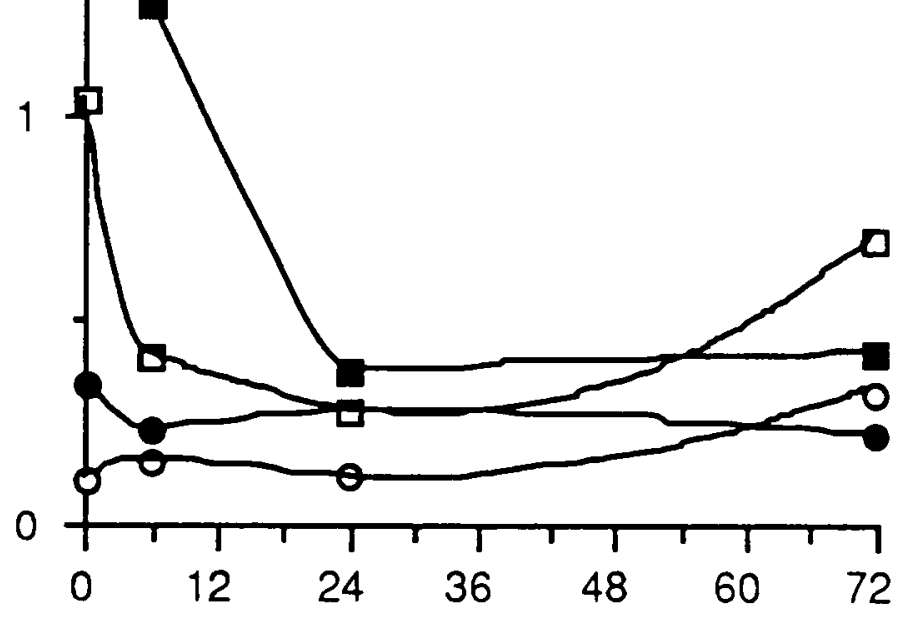

Time (h)

Fig. 4.4: Strain differences in the kinetics of uptake/binding of zymosan. EC from C57Bl/6 (closed symbols) and BALB/c (open symbols) were pulsed for 90 min with FITC-zymosan (a) or $1 \mu \mathrm{m}$ latex beads (b) at either $37^{\circ} \mathrm{C}$ (squares) or on ice (circles) at the culture times indicated. Analysis of LC by FACS was performed as before. Note that differences in intrinsic fluorescence between FITC-zymosan and fluorescent latex beads do not allow direct comparison of the index values in a) and b).

2 A surprising result is the apparent increase in phagocytosis (and binding) of latex beads by BALB/C LC from $24 \mathrm{~h}$ to $72 \mathrm{~h}$ (fig. $4.4 \mathrm{~b}$, open symbols). This was reproducible in $3 / 3$ independent experiments and was never seen with $\mathrm{C} 57 \mathrm{Bl} / 6 \mathrm{LC}$. The significance of these observations is not understood since, when assessed using long incubations, cultured LC were always less phagocytic than fresh ones. 


\section{5 - Splenic DC resemble cultured LC in their phagocytic ability.}

Splenic dendritic cells have traditionally been defined as non-phagocytic cells [55]. This has remained a puzzling observation in the context of antigen presentation given that so many particulate antigens are capable of eliciting a $\mathrm{T}$-dependent immune response. Our discovery that Langerhans cells (immature non-lymphoid DC) phagocytose antigens and retain them during maturation into non-phagocytic DC provides one solution to this conundrum. However, the observation that cultured LC still retain some residual phagocytic ability (fig. 4.2) led us to re-examine the capacity of splenic DC to phagocytose in vitro using the flow cytometric technique successfully utilised in the analysis of Langerhans cells. Murine splenic DC are traditionally isolated by means of a preparative method that includes an overnight incubation step, as originally described by Steinman [55]. Recently, however, three reports have suggested that overnight incubation may involve a maturational step similar to that undergone by LC in culture $[118,126,143]$. Therefore, DC-enriched low density adherent spleen cells (LODAC) were pulsed for 90 min with various particles before (d0) and after (d1) the overnight incubation step. DC within these cell populations were identified by staining with a DC-specific monoclonal antibody (N418 [75]) and particle association with DC was measured by double fluorescence flow cytometry.

Gated $\mathrm{N} 18^{+}$cells $(\mathrm{DC})$ associated with zymosan and with latex beads on day 0 and day 1 were compared to $6 \mathrm{~h}$ and $72 \mathrm{~h}$ cultured LC from the same mice (table 4.3 ). The percentage of DC associated with particles on both days may be slightly overestimated because N418 staining was not optimal (see chapter 2 for a profile) and low levels of contamination by phagocytic N418- cells (M $\varnothing$ ?) cannot be rigorously excluded. However, it is clear that lymphoid DC are capable of associating with particles and that this capacity decreases during overnight culture. Note that little decrease was seen for uptake of latex beads by LC from $6 \mathrm{~h}$ to $72 \mathrm{~h}$ (table 4.3 ; representative of three experiments). This is likely to be due to the fact that LC have already markedly down regulated the ability to ingest latex beads by 6 h (see fig. 4.4 and footnote 2 ). 
Table 4.3: Association of particles with fresh splenic DC decreases after overnight culture to become similar to that of cultured $L C$

\begin{tabular}{|c|c|c|c|c|c|c|}
\hline \multirow[b]{2}{*}{ Particle } & \multicolumn{3}{|c|}{ Fresh LC } & \multicolumn{3}{|c|}{ d0 DC } \\
\hline & $\begin{array}{l}\text { \% cells with } \\
\text { particles }\end{array}$ & $\begin{array}{c}\text { Mean } \\
\text { fluorescence } \\
\end{array}$ & Index & $\begin{array}{c}\text { \% cells with } \\
\text { particles }\end{array}$ & $\begin{array}{c}\text { Mean } \\
\text { fluorescence } \\
\end{array}$ & Index \\
\hline Zymosan & 83.1 & 906.3 & 75317 & 33.3 & 524.3 & 17461 \\
\hline $0.5 \mu \mathrm{m}$ latex & 96.2 & 82.8 & 7963 & 84.7 & 90.8 & 7692 \\
\hline $1 \mu \mathrm{m}$ latex & 25.7 & 163.1 & 4191 & 53.2 & 176.6 & 9397 \\
\hline \multirow[t]{3}{*}{$3 \mu \mathrm{m}$ latex } & 17.9 & 2976.0 & 53270 & 4.2 & 277.3 & 1165 \\
\hline & \multicolumn{3}{|c|}{ Cultured LC } & \multicolumn{3}{|c|}{ d1 DC } \\
\hline & $\begin{array}{c}c \text { cells with } \\
\text { particles }\end{array}$ & $\begin{array}{c}\text { Mean } \\
\text { fluorescence }\end{array}$ & Index & $\begin{array}{c}\% \text { cells with } \\
\text { particles }\end{array}$ & $\begin{array}{c}\text { Mean } \\
\text { fluorescence }\end{array}$ & Index \\
\hline Zymosan & 26.5 & 355.9 & 9431 & 16.1 & 518.8 & 8352 \\
\hline $0.5 \mu \mathrm{m}$ latex & 99.7 & 73.4 & 7322 & 48.8 & 50.8 & 2481 \\
\hline $1 \mu \mathrm{m}$ latex & 14.2 & 145.6 & 2068 & 10.4 & 123.0 & 1279 \\
\hline $3 \mu \mathrm{m}$ latex & 14.4 & 2220.7 & 31977 & 2.2 & 188.0 & 413 \\
\hline
\end{tabular}

EC suspensions and splenic LODAC were prepared from $20 \mathrm{C} 57 \mathrm{Bl} / 6$ mice and pulsed with the indicated particles for 90 min. Fresh and cultured LC were assayed at $6 \mathrm{~h}$ and $72 \mathrm{~h}$ of culture, respectively. d0 DC and d1 DC were assayed immediately after preparing LODAC (see methods) and 20h thereafter, respectively. Association of particles with LC and splenic DC was measured by double colour flow cytometry using B21.2 and N418 as the respective cell-specific markers.

It is conceivable that the decrease in particle association between day 0 and day 1 was due to inadvertent selection of different DC sub-populations because of differential adherence to tissue culture plastic on days 0 and 1 , particularly since it was necessary to wash the dishes quite vigorously to dislodge $\mathrm{d} 0 \mathrm{DC}$, even after $3.5 \mathrm{~h}$ incubation. To eliminate this potential source of artefact, low-density spleen cells (LOD) were not enriched further by adherence but, rather, were tested with particles before or after overnight culture on bacteriologic plastic. Essentially similar results were obtained (table 4.4; representative of three independent experiments), a decrease in association still occurring for all particles. Again, association values may be slightly inflated (see above), particularly since staining with N418 is less clear in LOD than in LODAC. Examination of 
the Petri dishes after recovering the cells on day 1 revealed quantitative recovery apart from a few M $\varnothing$ which remained bound. The association of DC and particles did not show strain differences such as those observed with LC (compare values for C57Bl/6 DC and those for $\mathrm{BALB} / \mathrm{C} \mathrm{DC}$ ), or at least differences that were reproducible between experiments. Similarly, no strain differences in DC association with FITC-S. aureus and FITC-C. parvum were observed in a separate experiment (not shown).

Table 4.4: Decrease in association of particles with DC during overnight culture is not an artefact from differential adherence to plastic and does not show strain differences

\begin{tabular}{|c|c|c|c|c|c|c|}
\hline \multirow[b]{3}{*}{ Particle } & \multicolumn{6}{|c|}{ Day 0} \\
\hline & \multicolumn{3}{|c|}{$\mathrm{C} 57 \mathrm{Bl} / 6$} & \multicolumn{3}{|c|}{$\mathrm{BALB} / \mathrm{c}$} \\
\hline & $\begin{array}{c}\% \text { cells with } \\
\text { particles }\end{array}$ & $\begin{array}{c}\text { Mean } \\
\text { fluorescence } \\
\end{array}$ & Index & $\begin{array}{c}\% \text { cells with } \\
\text { particles }\end{array}$ & $\begin{array}{c}\text { Mean } \\
\text { fluorescence } \\
\end{array}$ & Index \\
\hline Zymosan & 14.2 & 413.6 & 5873 & 15.5 & 424.2 & 6576 \\
\hline $0.5 \mu \mathrm{m}$ latex & 80.4 & 98.8 & 7946 & 69.7 & 93.9 & 6545 \\
\hline $1 \mu \mathrm{m}$ latex & 35.4 & 149.0 & 5276 & 28.2 & 145.5 & 4102 \\
\hline \multirow[t]{4}{*}{$3 \mu \mathrm{m}$ latex } & 1.4 & 423.8 & 593 & 4.5 & 311.3 & 1401 \\
\hline & \multicolumn{6}{|c|}{ Day 1} \\
\hline & \multicolumn{3}{|c|}{$\mathrm{C} 57 \mathrm{~B} 1 / 6$} & \multicolumn{3}{|c|}{$\mathrm{BALB} / \mathrm{c}$} \\
\hline & $\begin{array}{c}\% \text { cells with } \\
\text { particles }\end{array}$ & $\begin{array}{c}\text { Mean } \\
\text { fluorescence } \\
\end{array}$ & Index & $\begin{array}{c}\% \text { cells with } \\
\text { particles }\end{array}$ & $\begin{array}{c}\text { Mean } \\
\text { fluorescence }\end{array}$ & Index \\
\hline Zymosan & 6.1 & 390.0 & 2379 & 11.2 & 355.5 & 3981 \\
\hline $0.5 \mu \mathrm{m}$ latex & 44.5 & 47.7 & 2121 & 56.0 & 56.8 & 3183 \\
\hline $1 \mu \mathrm{m}$ latex & 7.3 & 127.7 & 932 & 9.0 & 126.5 & 1139 \\
\hline $3 \mu \mathrm{m}$ latex & 1.1 & 328.2 & 361 & 1.3 & 229.1 & 298 \\
\hline
\end{tabular}

Splenic LOD were incubated for $90 \mathrm{~min}$ with the indicated particles before or after overnight culture on bacteriologic plastic. Association of particles with DC was assayed by FACS after labelling with N418. Labelling with N418 was less clear than on LODAC populations and only the brightest cells were gated for analysis.

Microscopical examination of splenic DC was more difficult than with LC because N418 staining was not optimal and because of the abundance of $\mathrm{N}^{2} 8^{-}$, FITC ${ }^{+}$cells, but 
revealed that in many but not all cases particles appeared to be truly internalised. However. given that lymphoid DC have always been reported to be non-phagocytic, preparations of day 0 LODAC pulsed with zymosan or $1 \mu \mathrm{m}$ beads were prepared for immuno-electron microscopy to obtain more conclusive data on this issue. To differentiate DC from contaminating cell types, cryosections were labelled with N418 and 10nm gold particles using a three layer technique (see chapter 2). Phagocytosis by d0 DC (fig. 4.5a,b) had clearly occurred but was more limited in extent than that by contaminating N418phagocytes, presumed to be $\mathrm{M} \varnothing$ (fig. $4.5 \mathrm{c}$ ). The latter provided a clear internal negative control for the specificity of the staining procedure meaning that DC could be recognised purely on the basis of N418 positivity. This antigen was occasionally visualised within putative phagocytic vacuoles (fig. $4.5 \mathrm{~d}$ ).

Fig. 4.5: Immuno-EM analysis of phagocytosis by lymphoid DC. Uptake of a) zymosan (Z) and b) $1 \mu \mathrm{m}$ latex particles (LP) by splenic DC labelled with N418. Putative zymosanloaded macrophages (c) photographed on the same grid as the DC in a) show no gold particles and serve as an internal control for the specificity of staining. Other controls included omission of the first antibody and gave no staining (not shown). The area indicated by the arrows on the insets (magnification $\times 5800$ ) is enlarged on the main picture (magnification $\mathrm{x} 56000$ ). d) a putative phagocytic vesicle containing $1 \mu \mathrm{m}$ latex and labelled with N418 (magnification $x 56000$ ). 


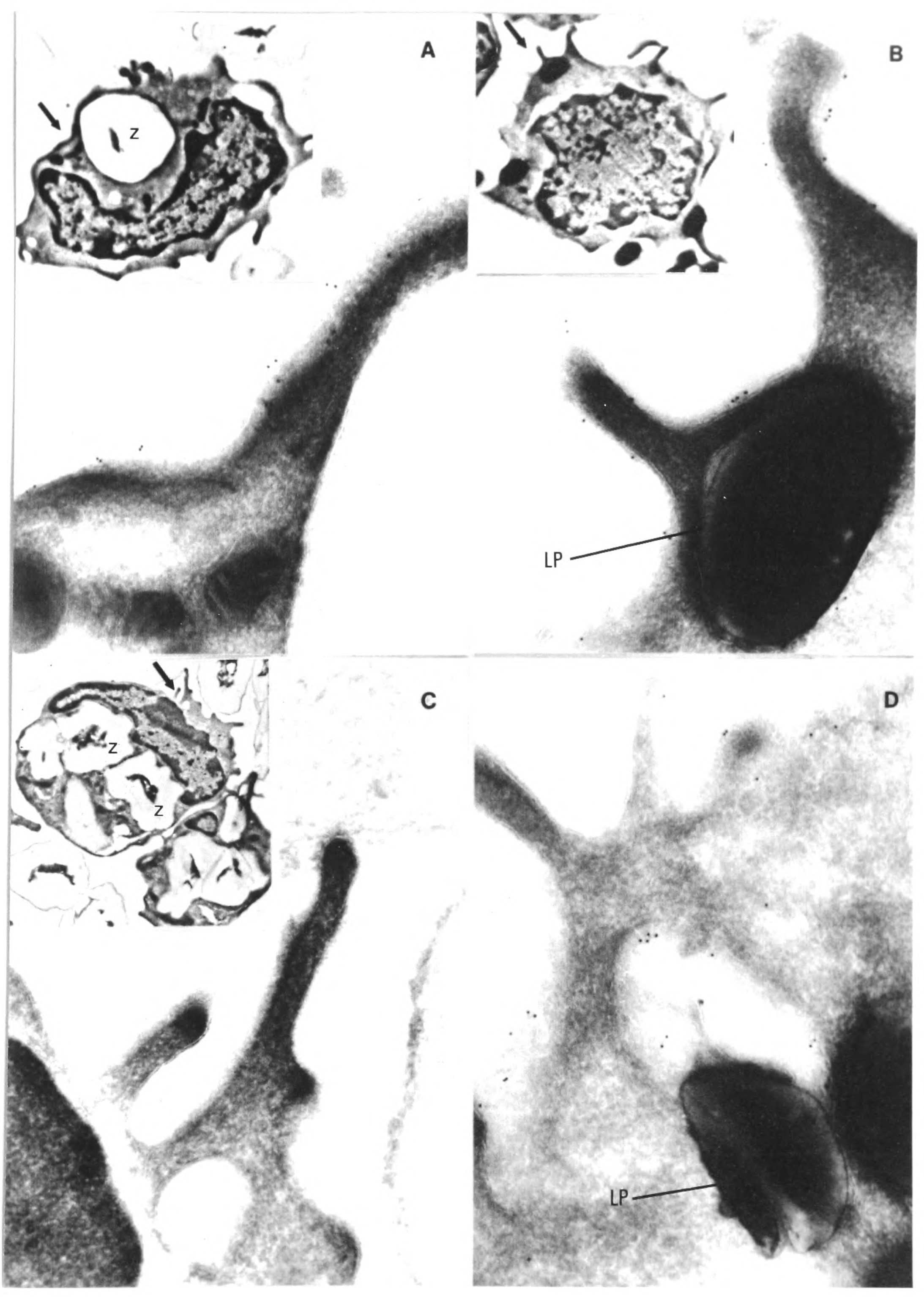


We conclude from these observations that at least a subset of freshly-isolated, immature, splenic DC have some phagocytic ability but, like that of fresh $\mathrm{LC}$, it is reduced after overnight culture, conceivably as part of the maturation process recently reported $[118,126,143]$. 


\section{6 - Summary}

This chapter has shown that cultured LC have markedly down regulated their potential to phagocytose $S$. aureus, $C$. parvum, zymosan or latex beads, in contrast to fresh LC (summarised in table 4.5). Migratory LC have an intermediate phagocytic capacity for zymosan and for latex beads as expected from the fact that they are more immature than cultured LC [104]. The kinetics of the down regulation are different in the C57BV/6 and the $\mathrm{BALB} / \mathrm{c}$ strains of mice with respect to zymosan uptake and this is likely to be due to strain differences in the expression of phagocytic receptors for this marker rather than because of intrinsic differences in the relative phagocytic ability of LC from the two strains. Fresh LC pulsed with zymosan do not regurgitate the particles but are capable of retaining them for at least 3 days, likely functioning to transport them to lymphoid tissues. We have not formally proven that antigen is degraded to peptides and displayed on MHC class II molecules but indirect evidence for degradation was obtained from flow cytometric assays on zymosan-pulsed LC.

Splenic DC showed a moderate capacity to internalise zymosan and latex beads on the day of isolation but became poorly phagocytic after $24 \mathrm{~h}$ in culture, in agreement with the results of Steinman and Cohn [55]. The relative capacity of d0 DC to internalise particulate antigens places them closer to migratory LC than to mature DC and is consistent with the hypothesis that the DC that are freshly isolated from the spleen of mice are not interdigitating cells but, rather, a subset of relatively immature, recently immigrated DC from non-lymphoid organs that are found in the marginal zone [118]. 
Table 4.5: Summary of studies on the phagocytic potential of Langerhans cells

\begin{tabular}{|c|c|c|c|}
\hline Particle & Fresh LC & Cultured LC & $\mathrm{M} \varnothing$ \\
\hline Zymosan & +++ & + & +++++ \\
\hline Zymosan + NMS & +++ & ND & $(+++++)$ \\
\hline S. cerevisiae & +++ & $\mathrm{ND}$ & $(+++++)$ \\
\hline $0.5 \mu \mathrm{m}$ latex beads & +++ & $+1-$ & +++++ \\
\hline $1 \mu \mathrm{m}$ latex beads & ++ & $+1-$ & +++++ \\
\hline $3 \mu \mathrm{m}$ latex beads & + & $+/-$ & +++++ \\
\hline C. parvum & ++ & $+1-$ & ++++ \\
\hline C. parvum + NMS & +++ & ND & $(+++++)$ \\
\hline S. aureus & +++ & + & ++++ \\
\hline S. aureus + NMS & +++ & ND & $(+++++)$ \\
\hline Colloidal carbon & - & ND & $(+++++)$ \\
\hline $\mathrm{SRBC} \pm \mathrm{IgG}$ & - & ND & $+++^{*}$ \\
\hline$S R B C \pm I g M$ & - & $\mathrm{ND}$ & - \\
\hline $\mathrm{SRBC} \pm \mathrm{C}^{\prime}$ & - & ND & $+1-^{*}$ \\
\hline Aldehyde-fixed SRBC & - & ND & +++++ \\
\hline FITC $-S R B C$ & - & ND & +++++ \\
\hline $\begin{array}{l}\text { Autologous RBC } \pm \\
\text { autologous serum }\end{array}$ & - & $\mathrm{ND}$ & $+1-$ \\
\hline
\end{tabular}

Compilations of results from different experiments. The plus and minus signs are used here solely as terms of comparison, unlike in table 3.3, and reflect a subjective view on part of the author. Symbols in parentheses for macrophages reflect published data on the phagocytic ability of these cells and, again, result from a subjective interpretation. Symbols not in parentheses reflect experiments done by the author. ND, not determined.

* Resident MØ. 


\section{Chapter 5}

\section{Receptors involved in phagocytosis of zymosan by Langerhans cells}

\section{1 - Introduction}

5.2 - Inhibition of zymosan uptake by LC

5.2.1 - Inhibition by soluble glycans

5.2.2 - Inhibition by anti-CR3

5.3 - Probing for mannose receptor on LC using soluble ligands

5.3.1 - Studies on the uptake of mannosylated BSA

5.3.2 - Failure to find a fluorescent ligand to measure binding to

MR rather than uptake

5.4 - Biochemical analysis of mannose receptor expression by $L C$ and MØ

5.4.1 - Characterisation of a rabbit antiserum against murine macrophage mannose receptor

5.4.2 - Anti-mouse MR antiserum does not inhibit receptormediated uptake of MR ligands and is a poor reagent for immunofluorescence

5.4.3 - Analysis of MR expression in LC by Western blotting

\section{5 - Summary}




\section{1 - Introduction}

Zymosan particles are yeast cell walls (Saccharomyces cerevisiae in these experiments) prepared by boiling yeast cells before and after extensive trypsin treatment [232]. The resulting particles consist essentially of pure carbohydrate and contain little residual protein [228]. Carbohydrate analysis reveals that zymosan is composed exclusively of a complex mixture of various chain length $\beta$-glucans, branched-chain polymers of $\mathrm{D}$-glucose linked by $\beta$-glucosidic linkages, and mannans, $\alpha$-linked polysaccharides of D-mannose (fig. 5.1).

At least three different receptors have been suggested to play a role in the ingestion of zymosan by mononuclear phagocytes and neutrophils based primarily on studies using specific inhibitors (table 5.1). These receptors will be discussed in turn.

Table 5.1: Receptors for zymosan in professional phagocytes

\begin{tabular}{|c|c|c|c|}
\hline Receptor & Phagocyte & Inhibitor ${ }^{1}$ & Reference $^{2}$ \\
\hline $\begin{array}{l}\text { Mannose receptor } \\
(\mathrm{MR})\end{array}$ & $\begin{array}{c}\text { Murine and human } \\
\text { MØ. Not on human } \\
\text { monocytes or } \\
\text { neutrophils }\end{array}$ & Mannan & $\begin{array}{c}{[223,224,230,233,} \\
234]\end{array}$ \\
\hline$\beta$-glucan receptor & $\begin{array}{c}\text { Human monocytes, } \\
\text { MØ and neutrophils. } \\
\text { Murine M } \varnothing\end{array}$ & $\beta$-glucan & {$[235-238]$} \\
\hline CR3 & $\begin{array}{c}\text { Human and murine } \\
\text { monocytes, M } \varnothing \text { and } \\
\text { neutrophils }\end{array}$ & $\begin{array}{l}\mathrm{M} 1 / 70 \text { and other } \\
\text { monoclonals }\end{array}$ & {$[223,224,239,240]$} \\
\hline
\end{tabular}

${ }^{1}$ Select inhibitors only.

2 Only references where specific use of zymosan was made are included. Not an exhaustive list. 


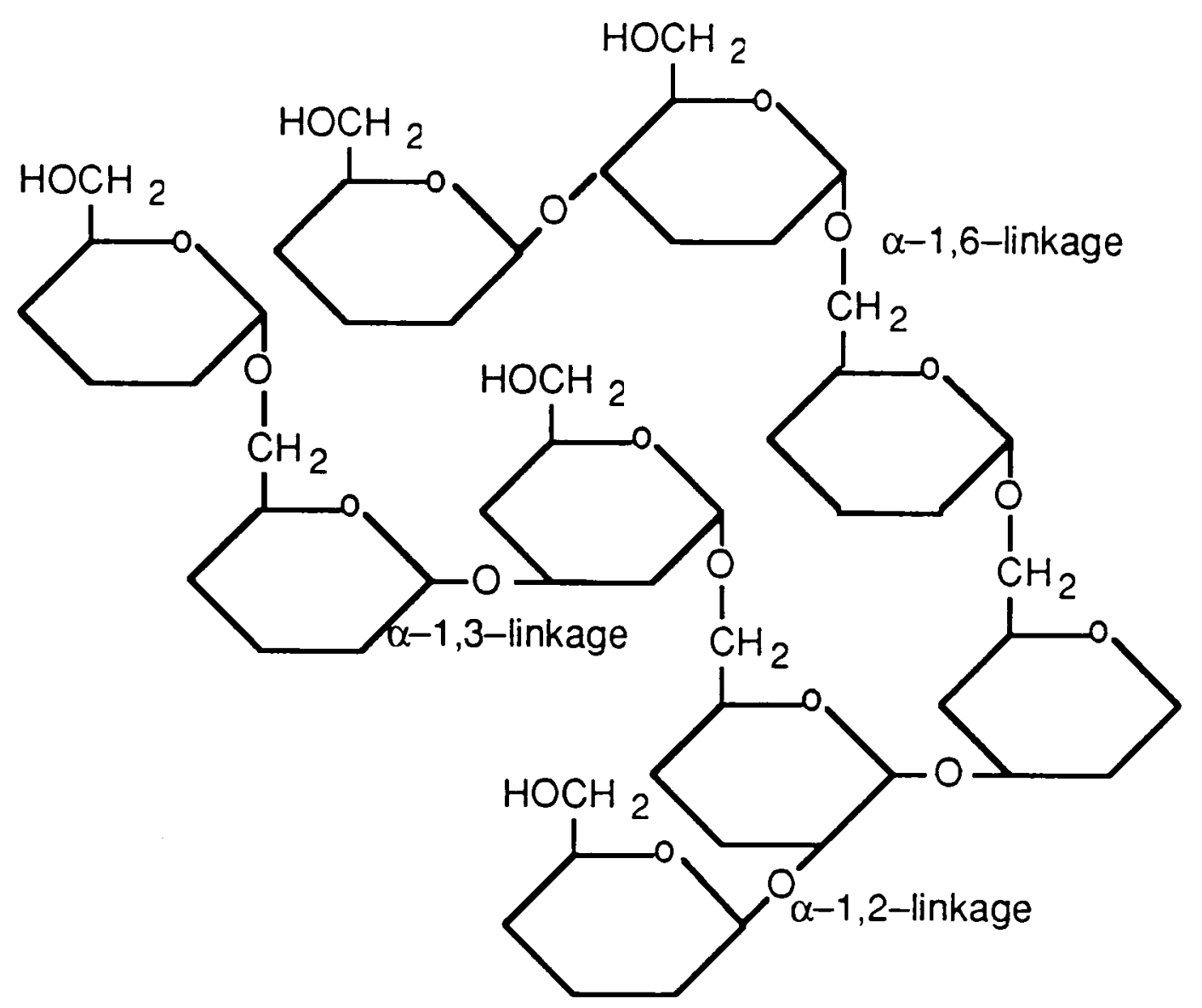

Mannan

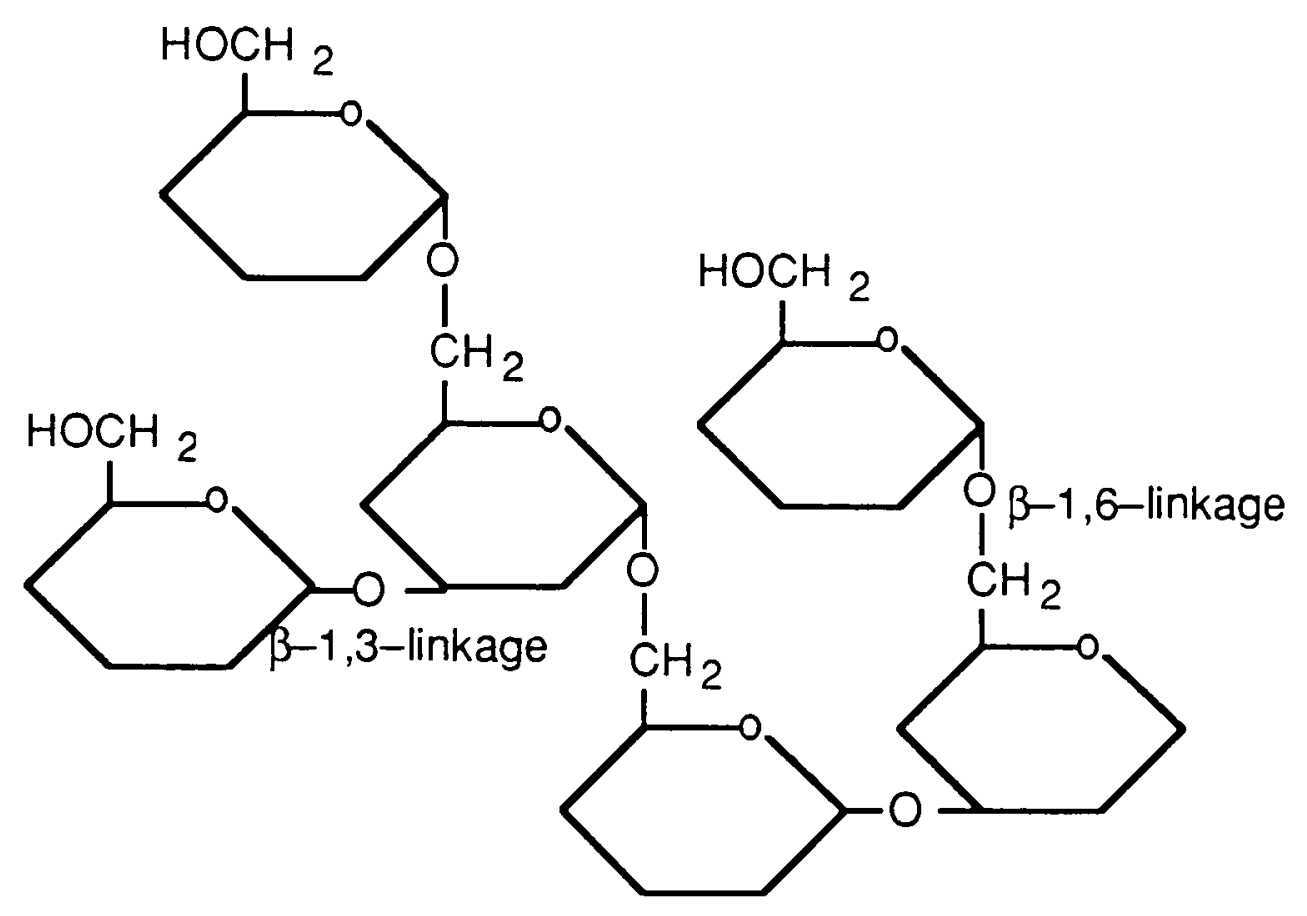

$\beta-$ glucan

Fig. 5.1: Composition of yeast mannan and $\beta$-glucan. $\beta$-glucan is a homopolymer of $\beta$ $1,6-$ linked $D$-glucose with $\beta-1,3$-linked branches arising at frequent intervals. Mannan is a $\alpha-1,6$-linked polymer of D-mannose with $\alpha-1,2-$ and $\alpha-1,3$-linked branches.

Perhaps the least controversial receptor for zymosan is the macrophage mannose receptor (MR), also known as the mannosyl-fucosyl receptor, which mediates endocytosis of a variety of glycoproteins with terminal mannose/ $\mathrm{N}$-acetylglucosamine residues 
(reviewed in references [241-244]). Early studies showed that the binding of Candida and zymosan by mouse alveolar M $\varnothing$ could be inhibited by mannose [233] and that mouse peritoneal $\mathrm{M} \varnothing$ plated on mannan-coated surfaces lost the ability to phagocytose zymosan but not IgG-coated erythrocytes [230]. In addition, M $\varnothing$ plated on immobilised zymosan no longer endocytosed mannosylated-BSA (ManBSA), a MR ligand, implying that the mannan-inhibitable phagocytic receptor for zymosan was the mannose receptor [245]. Specific confirmation that this is indeed the case was obtained recently by Ezekowitz and co-workers who transfected COS-1 cells with the cDNA for human MR and showed that these cells acquired the ability to internalise yeasts [202].

In addition to $M R$, another glycan receptor has been proposed to be involved in the phagocytosis of unopsonised zymosan. Studies on human monocytes (which lack MR) revealed the presence of a receptor that could be specifically inhibited by $\beta$-glucans [235] and that could mediate phagocytosis of $\beta$-glucan particles [246] in addition to zymosan [235]. $\beta$-glucan receptors have since been identified in other phagocytes such as murine Mø [236, 237, 247] and neutrophils [238] but, until recently, it was not clear whether they were a distinct receptor unit, particularly in the light of reports that CR3 may also have the capacity to bind $\beta$-glucans (see below). However, the two recognition units for $\beta$-glucan and C3bi are regulated independently $[238,248]$ and CR3 is relatively trypsin-resistant whereas the recognition unit for $\beta$-glucan is trypsin-sensitive [248]. This issue appears to be further resolved with the isolation of two $\beta$-glucan-binding membrane proteins of 160 and $180 \mathrm{kD}$ from a monocytic cell line and from human monocytes [249].

Inhibition with antibodies against the complement receptor type 3 (CR3), a member of the LFA-1 ( $\beta_{2}$ integrin) family, has clearly attributed a role for this molecule in the phagocytosis of zymosan $[223,224,239,240]$. What is less clear and the subject of controversy is the mechanism involved. The finding that $M \varnothing$ are capable of secreting fixed complement components led Ezekowitz and co-workers to suggest that CR3-mediated uptake of zymosan was due to local opsonisation with C3bi [223]. In line with this hypothesis, monoclonal antibodies against the C3bi binding site of human CR3 partly 
inhibited uptake of zymosan but those against a different epitope on the same molecule did not [224]. In contrast, Ross and colleagues, working with human neutrophils, found a monoclonal antibody that inhibited rosetting of C3bi-opsonised SRBC but had no effect on the binding or ingestion of yeast and another monoclonal which blocked binding and ingestion of yeasts but not binding of C3bi-coated erythrocytes [239]. These results were interpreted as evidence for a direct interaction of CR3 with sugars on the zymosan surface and a sugar ligand was later identified as $\beta$-glucan leading to the assertion that $C R 3$ and the $\beta$-glucan receptor were the same entity [240]. All these reports should be re-examined in the light of more recent data on the role of CR3 in phagocytosis (see chapter 1, section 1.3). Monoclonal antibody inhibition studies, although suggestive, should not be taken to mean a direct interaction of CR3 with zymosan since it is possible that their effect is mediated through interactions with another receptor [215]. The involvement of CR3 in phagocytosis/binding of zymosan by $\mathrm{M} \varnothing$ in the data by Ezekowitz [223, 224] could be due primarily to close interactions with other receptors (mannose and/or $\beta$-glucan). This is in line with the fact that CR3 by itself does not mediate phagocytosis of C3bi-coated erythrocytes in unstimulated $M \varnothing$ and, perhaps, helps explain the puzzling observation that inhibition of zymosan uptake with antibody against CR3 is only observed during the initial stage of the assay [223]. Similar interactions of CR3 with an autonomous $\beta$-glucan receptor might explain the data by Ross $[239,240]$ without the need to invoke a lectinbinding site (see also reference [238]). At present these issues remain unresolved.

Our observations on differences in the extent of zymosan phagocytosis between LC from three strains of mice strongly suggested there might be strain differences in the expression of the receptors mediating the phagocytosis of zymosan by these cells (section 3.4). This hypothesis was strengthened by the finding that down regulation of zymosan phagocytosis by $\mathrm{LC}$ followed different kinetics in the $\mathrm{C} 57 \mathrm{Bl} / 6$ and $\mathrm{BALB} / \mathrm{c}$ mouse strains and, in particular, that these differences correlated with differential kinetics of binding at $4^{\circ} \mathrm{C}$ (section 4.4). No strain differences were found with cultured LC (fig. 4.4), DC (table 4.4) or $M \varnothing$ (not shown) suggesting that this phenomenon was restricted to fresh 
Langerhans cells. This chapter presents results of experiments designed to define phagocytic receptors for zymosan on fresh Langerhans cells and to follow their fate during maturation of LC into non-phagocytic cells.

Besides CR3, none of the receptors that could mediate phagocytosis of zymosan have been described in dendritic cells. Mannose receptors are absent from rat veiled cells [89] but their possible expression on LC has not been investigated. Similarly, $\beta$-glucan binding proteins have not been described in DC. We used inhibition of zymosan uptake by the inhibitors listed in table 5.1 as an assay to investigate the potential presence and possible role of mannose, $\beta$-glucan and CR3 receptors in LC. Our results support the hypothesis that $\beta$-glucan and mannose receptors are expressed differentially on fresh LC from $\mathrm{C} 57 \mathrm{Bl} / 6$ and $\mathrm{BALB} / \mathrm{c}$ mice and that they mediate phagocytosis of zymosan by these cells, possibly without major involvement of CR3. 


\section{2 - Inhibition of zymosan uptake by LC}

\subsection{1 - Inhibition by soluble glycans}

Preliminary inhibition experiments with mannan and $\beta$-glucan suggested that mannose and $\beta$-glucan receptors were involved in phagocytosis of zymosan by fresh LC. One representative flow cytometry experiment is shown on fig. 5.2. Yeast mannan or $\beta$ glucan (from Sigma; see chapter 2, section 2.2.5), present throughout the incubation, inhibited uptake of zymosan by $54 \%$ and $67 \%$, respectively, in C57Bl/6 LC and their effect was specific in that phagocytosis of latex beads was not affected (fig. 5.2). Inhibition was apparent both in a reduction of percentage LC associated with zymosan and in the mean fluorescence of those cells (not shown). Dextran, a control polysaccharide, had no effect on the uptake of either zymosan or latex (fig. 5.2).

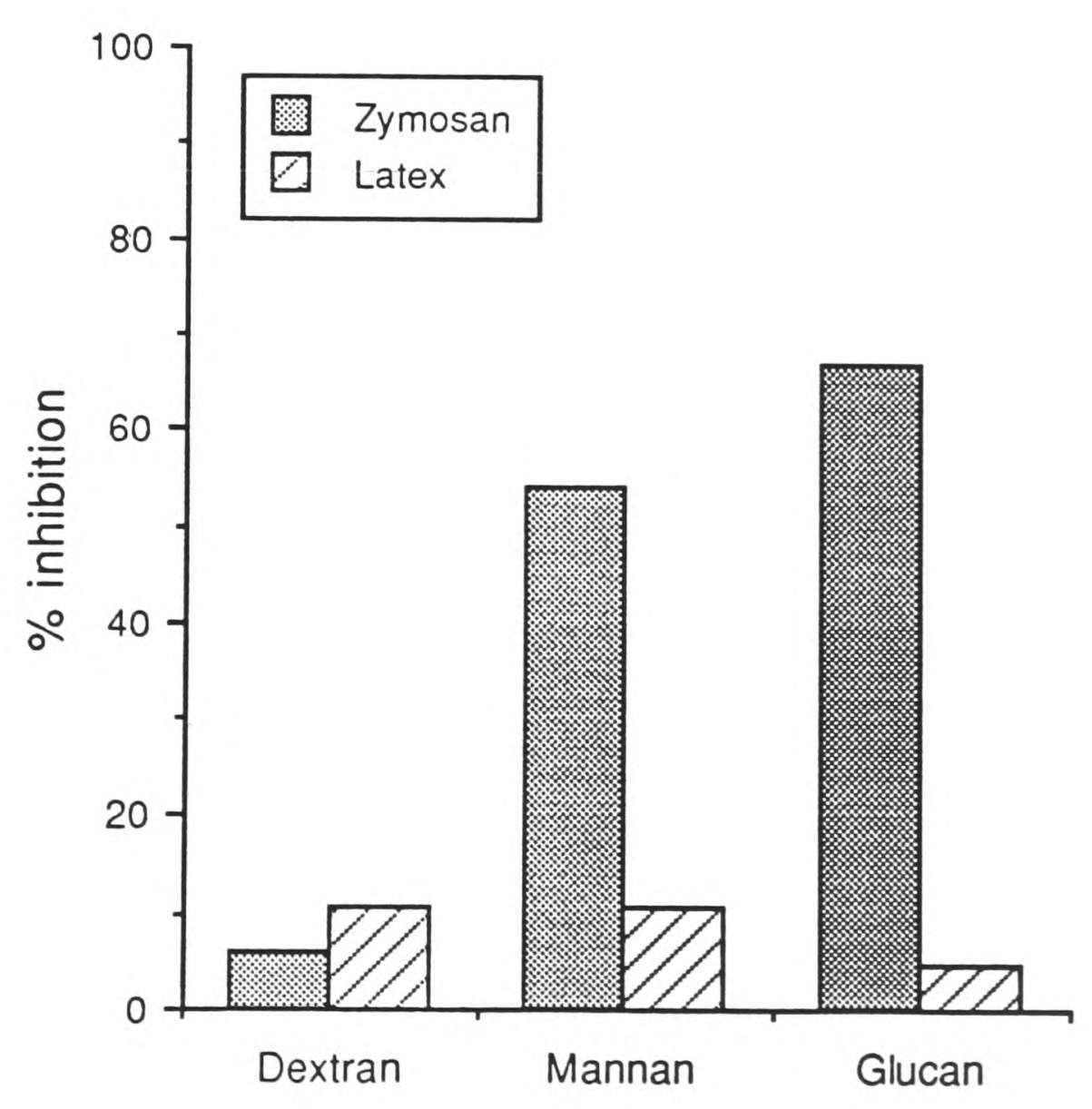

Fig. 5.2: Mannan and $\beta$-glucan inhibit phagocytosis of zymosan by LC. Fresh C57Bl/6 EC were incubated for $16 \mathrm{~h}$ with a saturating concentration of FITC-zymosan (shaded bars) or $1 \mu \mathrm{m}$ fluorescent latex beads (striped bars) in the presence or absence of the indicated 
glycans (Sigma; all at $1 \mathrm{mg} / \mathrm{ml}$ ). Particle association with LC was analysed by FACS as described in the methods. Results are expressed as index converted to percentage inhibition relative to a control incubated with R10 alone. Over $95 \%$ LC in the control samples had zymosan or latex. The apparent slight inhibition of latex bead uptake was at the level of mean fluorescence but not \% phagocytic LC.

When a range of concentrations of mannan and $\beta$-glucan were compared for their inhibitory effect, it was noted in three experiments that high concentrations of mannan were often required to achieve the same degree of inhibition obtained with low concentrations of $\beta$-glucan (not shown). Because commercially available yeast mannan is contaminated with $\beta$-glucan $[235,237]$, this finding raised the possibility that the inhibition obtained with mannan was due to interactions with a $\beta$-glucan receptor. Alternatively, because long incubations $(>12 \mathrm{~h})$ were routinely used it was possible that these differences reflected differential susceptibility of the two glycans to degradation. To address these points, incubation times were shortened to $90 \mathrm{~min}$ and highly purified mannan and $\beta$-glucan, a gift of Dr. V. Kery, Washington University, MO, U.S.A., were used instead of commercial preparations. The assays were performed after the cells had been cultured for $6 \mathrm{~h}$ since this was the time at which maximal phagocytosis of zymosan by C57Bl/6 LC was seen (fig. 4.4). The results (three experiments) confirmed that pure $\beta$ -glucan is indeed more potent, on a weight per volume basis, than pure mannan at inhibiting uptake of zymosan by 6h LC although both glycans inhibit in a dose-dependent manner (fig. 5.3). These results are consistent with reports that inhibition of phagocytosis via $\beta$-glucan receptors using $\beta$-glucan is achieved at low concentration (measured as weight/volume) $[235,237]$ compared to inhibition of $\operatorname{MR}$ by mannan $[223,230]$. However, strict comparisons of the inhibitory potency of the two glycans cannot be made without knowing their molecular weights and these are not available. ${ }^{1}$ Furthermore, the two glycans contain a complex mixture of polymers of different molecular weights, each of which is likely to contribute differentially to the inhibition observed $[237,250]$.

\footnotetext{
${ }^{1}$ In collaboration with Dr. V. Kery, Washington University, we attempted to determine the molecular weight of our preparations of mannan and glucan by HPLC but obtained a very broad peak and were unable to make worthwhile comparisons.
} 


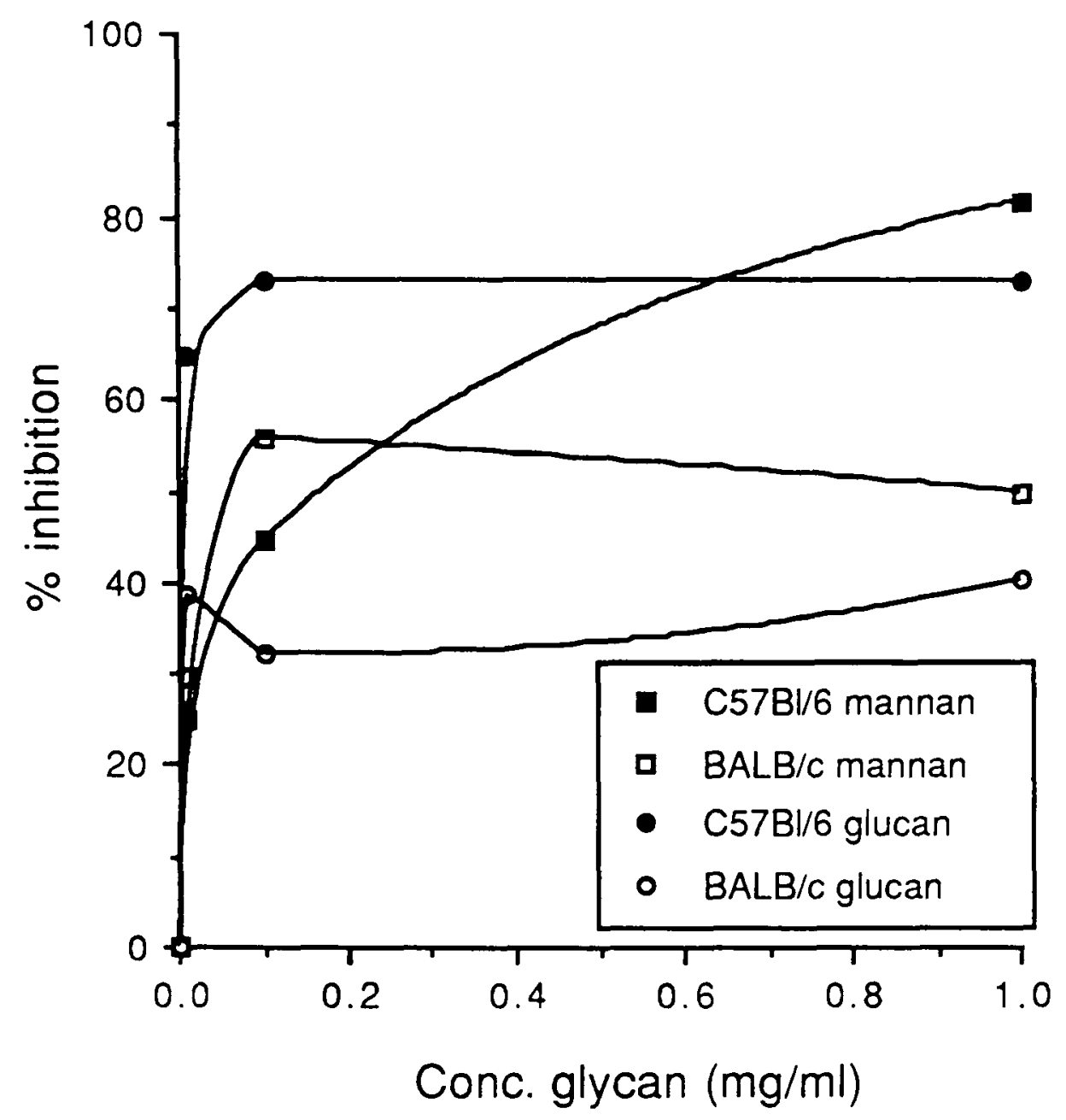

Fig. 5.3: Glycan inhibition of zymosan uptake by LC is dose-dependent. C57Bl/6 (closed symbols) and BALB/c (open symbols) epidermal cells cultured for 6 h were preincubated for $30 \mathrm{~min}$ at $37^{\circ} \mathrm{C}$ with the indicated concentrations of purified mannan (squares) or purified $\beta$-glucan (circles) and were pulsed with FITC-zymosan for 90 min in the continuous presence of glycan. FACS analysis of uptake by LC was performed as described in the methods. Results are expressed as the \% inhibition relative to the indices of control samples incubated without glycans. In this experiment, parallel samples were also treated with commercially available yeast mannan and $\beta$-glucan (Sigma) for comparison. Inhibition by these glycans was less efficient, particularly with $\beta$-glucan. Those results are omitted for the sake of simplicity.

At saturation, both glycans inhibited uptake by fresh LC from C57Bl/6 mice to a greater extent than by LC from BALB/c (fig. 5.3). This suggested that the functional expression of the relevant receptors might be greater in LC of the former strain. However, by $6 \mathrm{~h}$ of culture BALB/c LC have already markedly down regulated their ability to phagocytose zymosan, in contrast to $\mathrm{C} 57 \mathrm{Bl} / 6 \mathrm{LC}$ (fig. 4.4) and, indeed, the index for $\mathrm{BALB} / \mathrm{c} \mathrm{LC}$ in this experiment was three times lower than that for $\mathrm{C} 57 \mathrm{Bl} / 6 \mathrm{LC}$. When the 
assay was repeated at both $0 \mathrm{~h}$ and $6 \mathrm{~h}$ of culture (fig. 5.4), saturating concentrations of both glycans at $0 \mathrm{~h}$ still inhibited $\mathrm{C} 57 \mathrm{Bl} / 6 \mathrm{LC}$ to a greater extent than $\mathrm{BALB} / \mathrm{c}$ LC (fig. 5.4a) although the difference was less marked than at $6 \mathrm{~h}$ (fig. 5.4b). Dextran was used as a control and had no effect at either time point (fig. 5.4a,b). The greater inhibition of C57Bl/6 LC correlated directly with the greater extent of zymosan uptake by these cells compared to BALB/c LC (fig. 5.4c).
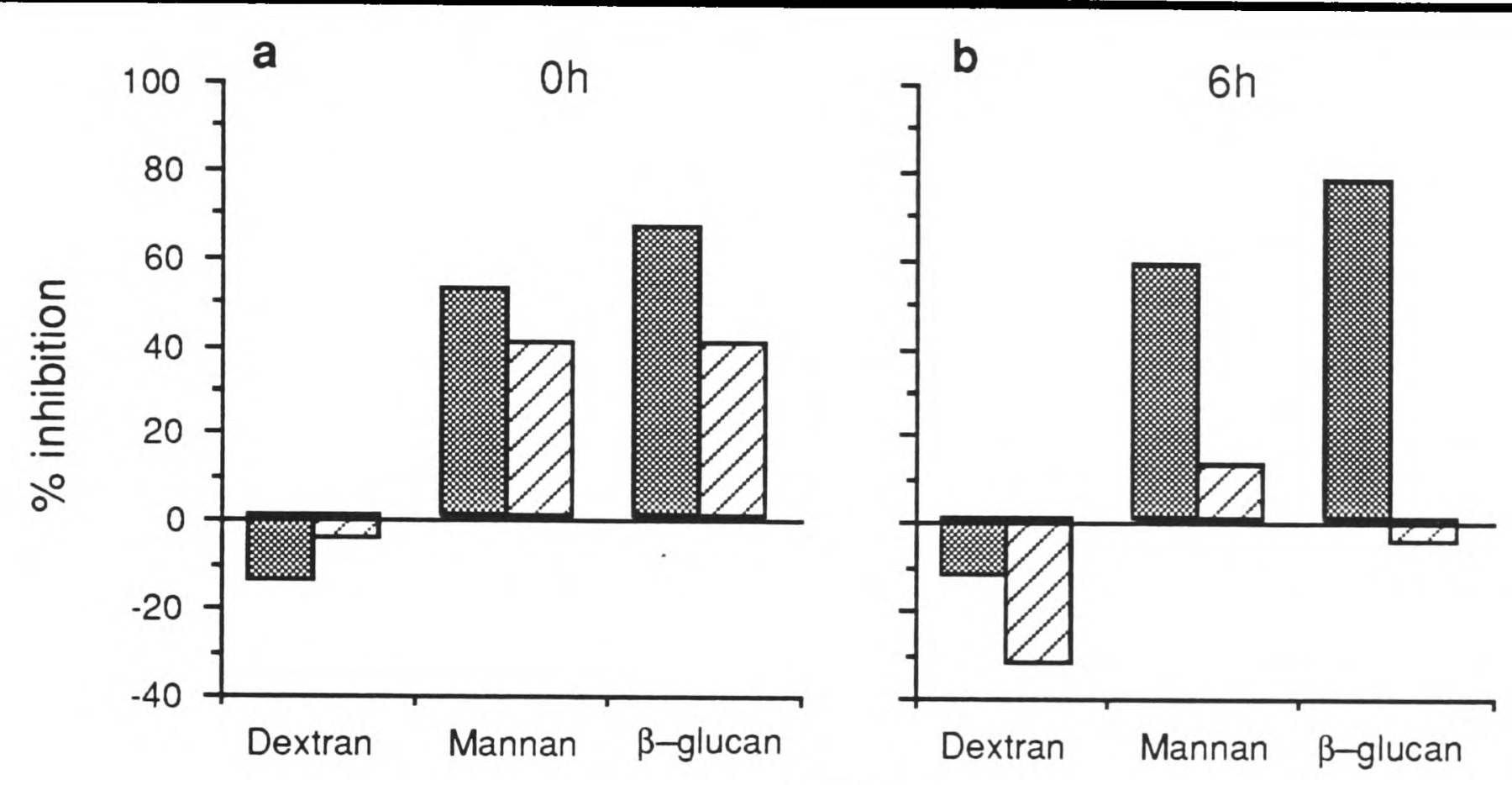

Inhibitor

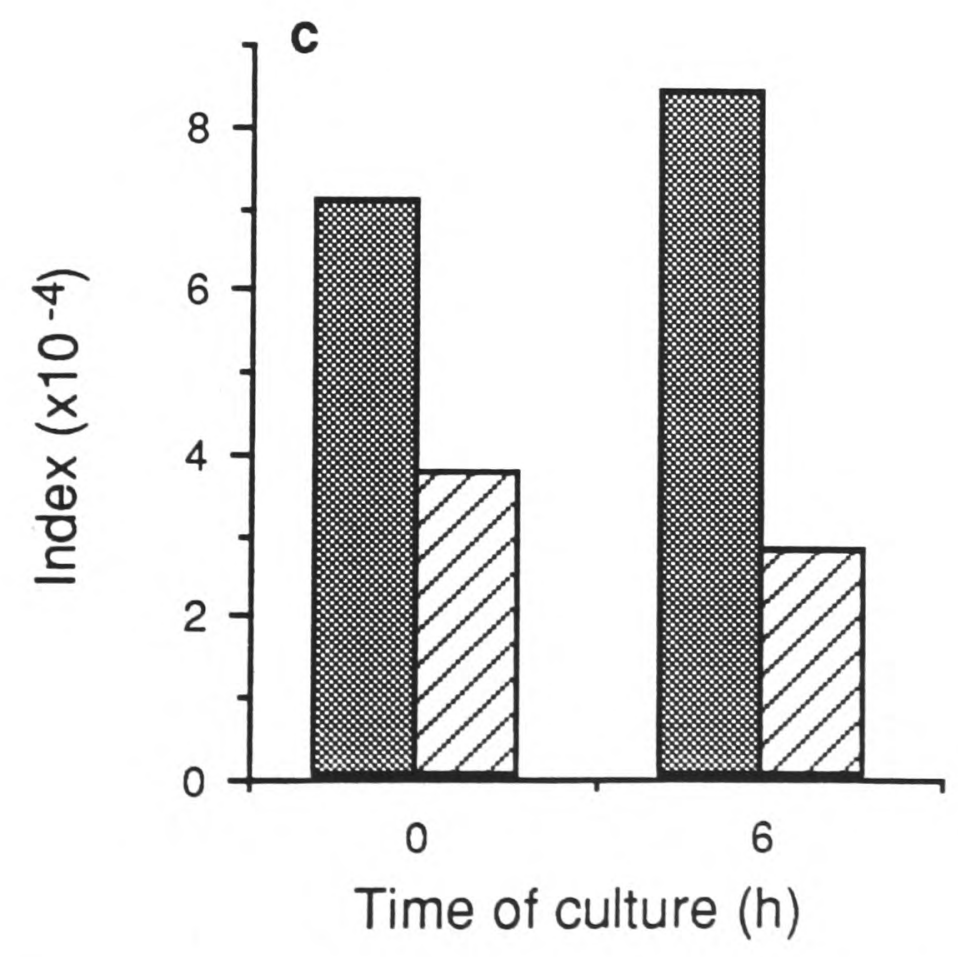

$\mathrm{C} 57 \mathrm{BI} / 6$

BALB/C

Fig. 5.4: Strain differences in the functional expression of $\beta$-glucan and mannose receptors by $\mathrm{LC}$. $\mathrm{C} 57 \mathrm{Bl} / 6$ (shaded bars) and $\mathrm{BALB} / \mathrm{c}$ (striped bars) EC cultured for a) $0 \mathrm{~h}$ 
or b) $6 \mathrm{~h}$ were pre-incubated for $30 \mathrm{~min}$ at $37^{\circ} \mathrm{C}$ with the indicated purified glycans (all at $1 \mathrm{mg} / \mathrm{ml}$ ) and were pulsed with FITC-zymosan for $90 \mathrm{~min}$ in the continuous presence of inhibitor. FACS analysis of uptake by LC was performed as described in the methods. Results are expressed as the \% inhibition relative to the indices of control samples incubated without glycans. c) index of control samples incubated in R10 alone. At $6 \mathrm{~h} \mathrm{b-}$ glucan no longer inhibited BALB/c LC in contrast to the previous experiment (fig. 5.3).

We conclude from this (fig. 5.4) and the previous (fig. 5.3) experiments that the functional expression of receptors recognising mannose and $\beta$-glucan is regulated differentially in $\mathrm{C} 57 \mathrm{~B} 1 / 6$ and $\mathrm{BALB} / \mathrm{C}$ LC. Using the extent of inhibition by glycan as a measure of the functional level of expression of the receptors, C57Bl/6 LC have greater levels of both and they increase from 0 to $6 \mathrm{~h}$ of culture in parallel with increased phagocytosis of zymosan. In contrast, functional expression of receptors recognising mannose and $\beta$-glucan in $\mathrm{BALB} / \mathrm{C} \mathrm{LC}$ is lower than in $\mathrm{C} 57 \mathrm{Bl} / 6 \mathrm{LC}$ at $0 \mathrm{~h}$ and decreases further by 6 h of culture (fig. $5.4 \mathrm{a}, \mathrm{b}$ ). One discordant result should be reported: when the inhibition assays lasted for the initial 12-16h of culture (rather than $90 \mathrm{~min}$ assays at given culture times, as above), mannan (in one of three experiments) and $\beta$-glucan (in two of three experiments) inhibited zymosan uptake by BALB/c LC to a greater extent than by $\mathrm{C} 57 \mathrm{Bl} / 6 \mathrm{LC}$, in spite of the fact that the latter cells were more phagocytic for zymosan in all three experiments.

If, indeed, there is a correlation between the capacity to phagocytose zymosan via glycan receptors and their functional expression levels, one might expect that after the initial rise from $0 \mathrm{~h}$ to $6 \mathrm{~h}$ (fig. $5.4 \mathrm{a}, \mathrm{b}$ ), inhibition levels would drop concomitantly with down regulation of zymosan uptake (fig. 4.4). In keeping with this hypothesis, the inhibitory effect of glycans was rapidly lost during culture (fig. 5.5) and the residual uptake seen at $72 \mathrm{~h}$ was not inhibitable by $\beta$-glucan or mannan. ${ }^{2}$ These results could be interpreted to suggest that both a mannose receptor and a $\beta$-glucan receptor present on fresh LC are

\footnotetext{
2 In one experiment, zymosan uptake by $\mathrm{C} 57 \mathrm{~B} 1 / 6 \mathrm{LC}$ was inhibitable by mannan after culture. However, the dose of zymosan employed was $30 x$ lower than usual and the maximum percentage of phagocytosing LC at $6 \mathrm{~h}$ was only $27.5 \%$.
} 
down regulated during maturation. Further evidence for down regulation of mannose receptor expression during culture is presented later in this chapter.

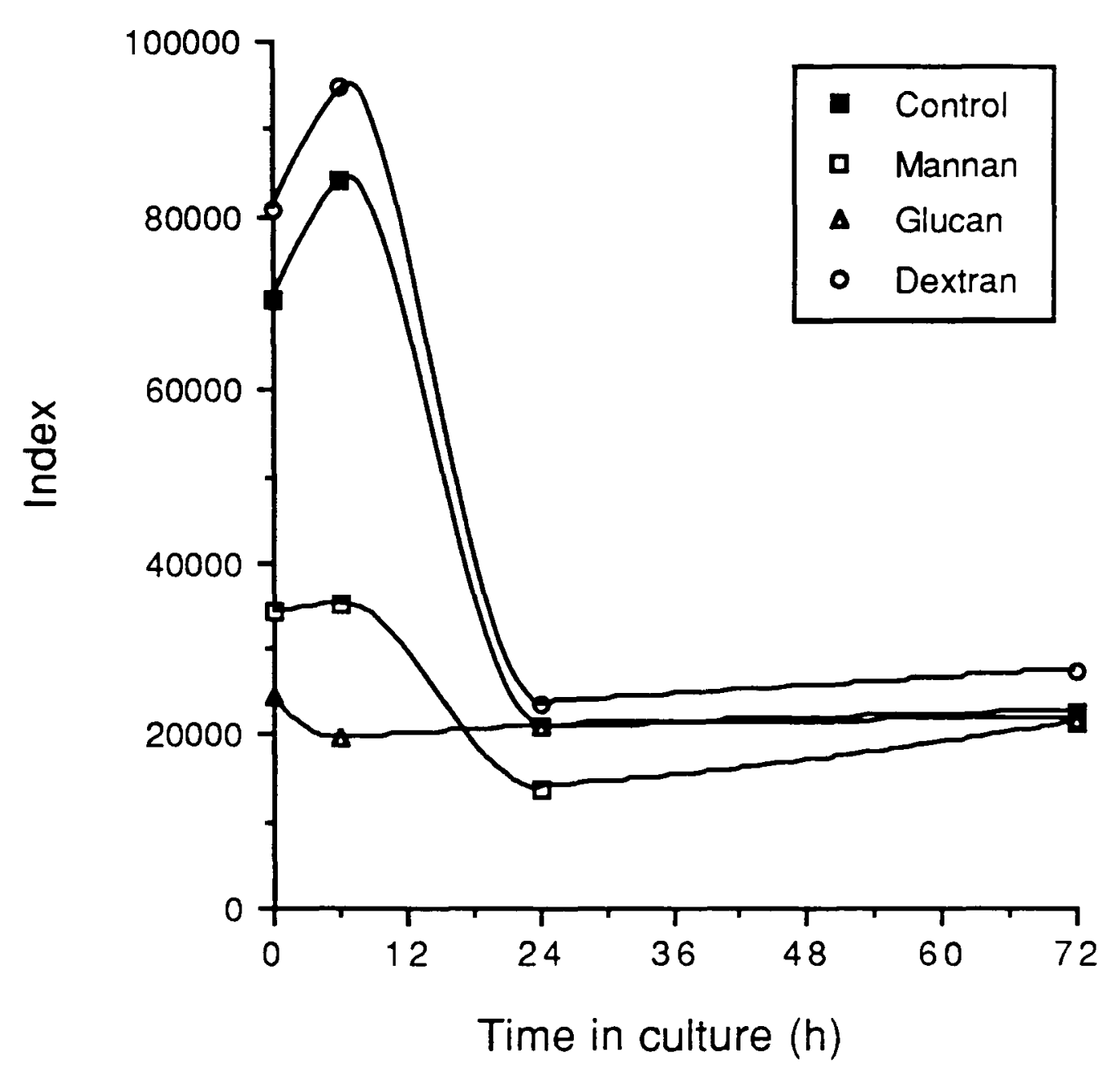

Fig. 5.5: Inhibition of zymosan uptake by mannan and $\beta$-glucan is lost with down regulation of uptake. The experiment was performed as in fig. 5.4 using C57Bl/6 EC cultured for $0,6,24$ and $72 \mathrm{~h}$. Results are expressed as the index obtained from FACS analysis.

We conclude from the data presented in this section that both mannan- and $\beta$ glucan-recognition units are present on fresh Langerhans cells and play a role in the phagocytosis of zymosan but not that of latex particles by these cells. LC from C57Bl/6 and BALB/c mice differ in the extent to which phagocytosis of zymosan can be inhibited by mannan and $\beta$-glucan either because of qualitative or quantitative differences in functional expression of these receptors. In contrast to LC, M $\varnothing$ did not show strain differences in the extent inhibition by mannan (table 5.2) or by $\beta$-glucan (not shown). 
Based on the level of inhibition that can be achieved at different times in culture it is speculated that receptors for mannan and $\beta$-glucan are down regulated during LC maturation but any causal relationship to down regulation of zymosan uptake remains undetermined.

Table 5.2: $M \emptyset$ do not show strain differences in inhibiton by mannan

\begin{tabular}{cccccc}
\hline & \multicolumn{2}{c}{ C57Bl/6 } & & \multicolumn{2}{c}{ BALB/c } \\
\cline { 2 - 3 } \cline { 5 - 5 } Inhibitor & \% uptake & fluorescence & & \% uptake & $\begin{array}{c}\text { Mean } \\
\text { fluorescence }\end{array}$ \\
\hline None & 99.0 & 1594.94 & & 98.6 & 1696.94 \\
Mannan & 94.1 & 1256.07 & & 93.7 & 1334.17 \\
& & & & $\mathbf{2 5 . 1 \%}$ \\
Inhibition & & $\mathbf{2 5 . 3 \%}$ & &
\end{tabular}

Thioglycollate-induced $M \varnothing$ were pre-incubated for $1 \mathrm{~h}$ with or without mannan $(10 \mathrm{mg} / \mathrm{ml})$ before being challenged with a saturating dose of FITCzymosan for $12 \mathrm{~h}$ in the constant presence or absence of inhibitor. Uptake was analysed by single colour flow cytometry. Inhibition by mannan (bold) is expressed as a percentage relative to the index of the control sample. Note that the extent of uptake of zymosan by $\mathrm{BALB} / \mathrm{c}$ and $\mathrm{C} 57 \mathrm{Bl} / 6 \mathrm{M} \varnothing$ is virtually the same (compare \% uptake and mean fluorescence between strains) as mentioned in chapter 3 . Results are representative of three independent experiments with variable extent of inhibition.

Although it is convenient to talk about mannose and $\beta$-glucan receptors in LC it is important to note that our data do not indicate that these are the same receptors which are involved in the uptake of zymosan by neutrophils and/or mononuclear phagocytes. It is possible that entirely different receptors with the same ligand specificity are involved or, indeed, that the two recognition units identified represent a single receptor with multiple ligand specificities. For example, in many of the experiments described above, a puzzling observation was that greater than $50 \%$ inhibition of zymosan uptake by $\mathrm{C} 57 \mathrm{Bl} / 6 \mathrm{LC}$ could be achieved with each purified glycan at saturation (fig. 5.3, closed symbols; fig. 5.4a,b shaded bars, fig. 5.5). Since cross-contamination of the glycans was ruled out by carbohydrate analysis (V. Kery, personal communication) this finding suggested that a single receptor might be involved, with specificity for both mannan and $\beta$-glucan, or that 
independent mannan and $\beta$-glucan receptors act synergistically to promote zymosan phagocytosis. Alternatively, it is possible that both mannan and $\beta$-glucan interact with a third receptor to exert part of their effect. One obvious candidate would be CR3 (see sections 1.3 .2 and 5.1).

\subsection{2: Inhibition by anti-CR3}

Only preliminary studies were carried out with antibodies against complement receptor type 3 and, at present, we have no evidence for involvement of this molecule in phagocytosis of zymosan by LC. Initial results suggested that M1/70 did partially inhibit zymosan uptake by both $\mathrm{BALB} / \mathrm{c}$ and $\mathrm{C} 57 \mathrm{Bl} / 6 \mathrm{LC}$ whereas $5 \mathrm{C} 6$, recognising a different epitope on the same molecule [189], did not (table 5.3), in agreement with the fact that 5C6 does not inhibit uptake of zymosan by mouse MØ (M. Stein, personal communication). However, M1/70 was used as a culture supernatant in these experiments whereas 5C6 was diluted from a stock of purified antibody (a generous gift from Prof. S. Gordon, Sir William Dunn School of Pathology, Oxford). F4/80 supernatant was also inhibitory suggesting that non-specific effects were associated with the use of supernatants during such a long incubation (table 5.3).

Table 5.3: Apparent inhibition of zymosan uptake by M1/70 supernatant

\begin{tabular}{ccc}
\hline & \multicolumn{2}{c}{ \% inhibition } \\
\cline { 2 - 3 } Antibody & BALB/c & C57B1/6 \\
\hline 5 C6 & 10.30 & 3.9 \\
M1/70 & 65.08 & 32.8 \\
F4/80 & 37.42 & 43.9 \\
\hline
\end{tabular}

$\mathrm{EC}$ from $\mathrm{C} 57 \mathrm{~B} 1 / 6$ or $\mathrm{BALB} / \mathrm{c}$ mice were pre-incubated with the indicated antibodies for 60 min before challenge with FITC-zymosan for $12 \mathrm{~h}$. M1/70 and F4/80 were used as culture supernatants diluted 1:2 in R10; $5 \mathrm{C} 6$ was used at $20 \mu \mathrm{g} / \mathrm{ml}$ in R10. Analysis of LC was by FACS as in methods. Results are translated into percentage inhibition relative to the index of control LC incubated in R10 alone. 
These problems were addressed with the use of purified M1/70 at a defined concentration. In a 45 min binding assay on ice, both $\mathrm{M} 1 / 70$ and $5 \mathrm{C} 6$ were almost saturating at $1 \mu \mathrm{g} / \mathrm{ml}$ and were completely saturating at $5 \mu \mathrm{g} / \mathrm{ml}$ for $10^{6}$ resident peritoneal $\mathrm{M} \varnothing$ (not shown). We used $10-40 \mu \mathrm{g} / \mathrm{ml}$ of antibody in an inhibition assay on C57Bl/6 LC and were unable to detect inhibition of zymosan uptake although a positive control sample incubated with mannan was readily inhibited (table 5.4).

Table 5.4: Purified M1170 fails to inhibit uptake of zymosan by $L C$

\begin{tabular}{ccc}
\hline Inhibitor & Conc $(\mu \mathrm{g} / \mathrm{ml})$ & $\begin{array}{c}\text { Percentage of } \\
\text { control index }\end{array}$ \\
\hline None & - & 100.0 \\
M1/70 & 40 & 114.3 \\
M1/70 & 20 & 110.0 \\
M1/70 & 10 & 115.9 \\
$5 C 6$ & 40 & 101.2 \\
5C6 & 20 & 104.5 \\
5C6 & 10 & 106.1 \\
Mannan & 2000 & 52.4 \\
\hline Fresh C57Bl/6 LC were incubated for 16h with \\
a saturating amount of FITC-zymosan in the \\
presence or absence of the indicated inhibitors. \\
Association with particles was measured by \\
FACS. Results are expressed as percentage of \\
the index of a control sample incubated \\
without inhibitors. 88.3\% of LC were \\
phagocytic in the control sample.
\end{tabular}

Very recent work on infection of LC by Leishmania major suggests that parasite entry can be inhibited by including purified M1/70 IgG during a $24 \mathrm{~h}$ infection assay ([251] and $\mathrm{H}$ Moll, personal communication). In those experiments, M1/70 was used at $200 \mu \mathrm{g} / \mathrm{ml}$ and, therefore, we tried this concentration of antibody, vastly in excess of saturation, to attempt to inhibit uptake of zymosan during a $16 \mathrm{~h}$ assay. However, in two experiments with $\mathrm{C} 57 \mathrm{Bl} / 6 \mathrm{LC}$ and one experiment with $\mathrm{BALB} / \mathrm{C} \mathrm{LC}$ inhibition was not seen with either $\mathrm{M} 1 / 70$ or $5 \mathrm{C} 6$ at $200 \mu \mathrm{g} / \mathrm{ml}$ (not shown; results as in table 5.4 ); in all experiments uptake 
was readily inhibitable by mannan (not shown; results as in table 5.4) and, in one experiment where $\beta$-glucan and dextran were also included, the former but not the latter glycan was also inhibitory, as in the previous section. Perhaps worryingly, we were also unable to inhibit uptake of zymosan by $\mathrm{M} \varnothing$ with $\mathrm{M} 1 / 70$. M1/70 or $5 \mathrm{C} 6$, ranging in concentration from 10 to $200 \mu \mathrm{g} / \mathrm{ml}$, had either no effect or effectively increased uptake of zymosan by resident peritoneal $\mathrm{M} \emptyset$ or thio-M $\varnothing$ (table 5.5). These results are representative of eight independent experiments using either resident or thioglycollateinduced macrophages and testing different incubation times, ranging from $10 \mathrm{~min}$ to $16 \mathrm{~h}$. Several combinations of mannan, $\beta$-glucan and M1/70 also failed to significantly inhibit uptake by thio-M $\varnothing$ (not shown). It was possible that, if CR3 was involved in phagocytosis but not binding of zymosan, as suggested [215] (see chapter 1, section 1.3), the lack of inhibition seen with $\mathrm{M} 1 / 70$ could be due to the inability of the flow cytometry assay to distinguish between internalised and bound particles. This proved not to be the case since similar results were obtained when the fluorescence of external particles was quenched with trypan blue in two experiments (table 5.5). 
Table 5.5: Lack of inhibition seen with M1/70 is not due to the inability to differentiate between bound and internalised particles

\begin{tabular}{cccc}
\hline Inhibitor & Conc $(\mu \mathrm{g} / \mathrm{ml})$ & Total & Internalised \\
\hline None & - & 100.0 & 100.0 \\
Mannnan & 1000 & 88.3 & 104.0 \\
B-glucan & 1000 & 86.0 & 88.8 \\
Dextran & 1000 & 98.0 & 101.1 \\
M1/70 & 10 & 110.2 & 117.3 \\
M1/70 & 1 & 106.1 & 112.6 \\
M1/70 & 0.1 & 90.7 & 95.6 \\
M1/70 & 0.01 & 87.1 & 88.2 \\
5C6 & 10 & 88.8 & 100.6 \\
5C6 & 1 & 95.6 & 107.2 \\
5C6 & 0.1 & 88.9 & 99.5 \\
5C6 & 0.01 & 86.1 & 92.0 \\
\hline
\end{tabular}

Resident peritoneal cells were plated on Petri dishes in R10 for $2 \mathrm{~h}$ and non-adherent cells were discarded. MØ were detached from the dishes by vigorous washing with cold $5 \mathrm{mM}$ EDTA in PBS and were pre-incubated for $5 \mathrm{~min}$ at $37^{\circ} \mathrm{C}$ with the indicated inhibitors in R10. FITC-zymosan was added for a further $10 \mathrm{~min}$ in the constant presence of inhibitor. The assay was terminated by fixation on ice. Association with particles was measured by FACS before (total) or after (internalised) adding trypan blue $(0.02 \%)$. Results are expressed as a percentage of the index of a control sample incubated without inhibitors. $70.5 \% \mathrm{M} \varnothing$ in the control sample were associated with zymosan (mean fluorescence 2179.95 ) and $64.1 \%$ had internalised the particles (mean fluorescence 920.59). Note that trypan blue does not penetrate cells fixed in formaldehyde (personal observations). Quenching of FITC-zymosan fluorescence with trypan blue is not complete (see fig. 2.5) but is sufficient to discriminate between bound and ingested particles.

It is difficult to explain why M1/70 or, indeed, mannan and $\beta$-glucan did not have any significant inhibitory effect on uptake of zymosan by peritoneal $\mathrm{M} \emptyset$ even during a 10 min assay (table 5.5). Mannan and M1/70 have been shown to inhibit uptake of zymosan by both resident and thio-M $\varnothing$ in short assays [223]. Similarly, mouse bone marrowderived $\mathrm{M} \varnothing$, resident peritoneal $\mathrm{M} \varnothing$ and thio-M $\mathrm{M} \varnothing$ contain $\beta$-glucan receptors which are 
active in zymosan phagocytosis $[236,237]$. The discrepancy between our results and those obtained by other groups cannot be due to methodological differences since uptake of zymosan by J774E M $\varnothing$ was readily inhibited by mannan or $\beta$-glucan during a 90 min flow cytometry assay (see below, table 5.6). Neither can it be attributed to trivial explanations such as loss of activity or batch variability in our stocks of mannan and $\beta$-glucan since the same stocks always inhibited uptake of zymosan by Langerhans cells (table 5.4 and data not shown).

We conclude from these preliminary data that uptake of zymosan by Langerhans cells cannot be inhibited with a vast excess of anti-CR3 monoclonal although it is readily inhibited by mannan and $\beta$-glucan in both the $\mathrm{C} 57 \mathrm{Bl} / 6$ and $\mathrm{BALB} / \mathrm{C}$ strains (table 5.4 and data not shown; see text). However, we were also unable to inhibit uptake by resident M $\varnothing$ with the same antibodies (table 5.5 and data not shown; see text), and, therefore, did not have a positive control for anti-CR3-inhibitable phagocytosis of zymosan. ${ }^{3}$

\section{3 - Probing for mannose receptor on LC using soluble ligands}

The results on inhibition of zymosan uptake by mannan and $\beta$-glucan suggested that a mannose and a $\beta$-glucan receptor might be expressed by fresh $\mathrm{LC}$ and be down regulated during maturation. Our bias was that these receptors might be the same as those expressed by professional phagocytes, and we concentrated on the macrophage mannose receptor to follow up these observations. Although no monoclonal antibody is yet available against murine $M R$, it is more amenable to study than the monocyte $\beta$-glucan receptor about which little information exists. The human mannose receptor has been purified, cloned, sequenced and its function has been related to its structure (reviewed in reference [244]). It is a $165-178 \mathrm{kD}$ C-type animal lectin [252] that functions in receptor-mediated endocytosis and phagocytosis and is primarily restricted to cells of the reticuloendothelial system. It binds sugars (mannose $\approx$ fucose $>N$-acetyl-glucosamine $\geq$ glucose $>$ xylose $>>>$

\footnotetext{
${ }^{3}$ Although we established that the antibodies showed saturable binding to MØ membranes (see text).
} 
galactose) in a $\mathrm{pH}-$ and $\mathrm{Ca}^{2+}-$ dependent fashion and has highest affinity for branched, polyvalent ligands. Structurally, it contains eight carbohydrate-recognition domains (CRDs) [202, 253], three of which are required for high-affinity binding to multivalent ligands and only one of which (CRD4) is responsible for the multiple ligand specificity [254] (fig. 5.6). Mannose receptor is an efficient endocytic receptor and is internalised constitutively via coated pits. It recycles rapidly from early endosomes and, at steady-state, more than $80 \%$ of the receptor is intracellular [255, 256]. Less is known about its function in phagocytosis but it is involved in the uptake of yeasts [202, 233], Leishmania donovani promastigotes [257] and Pneumocystis carinii [203], in addition to zymosan [223, 224, $230,233,234,245]$.

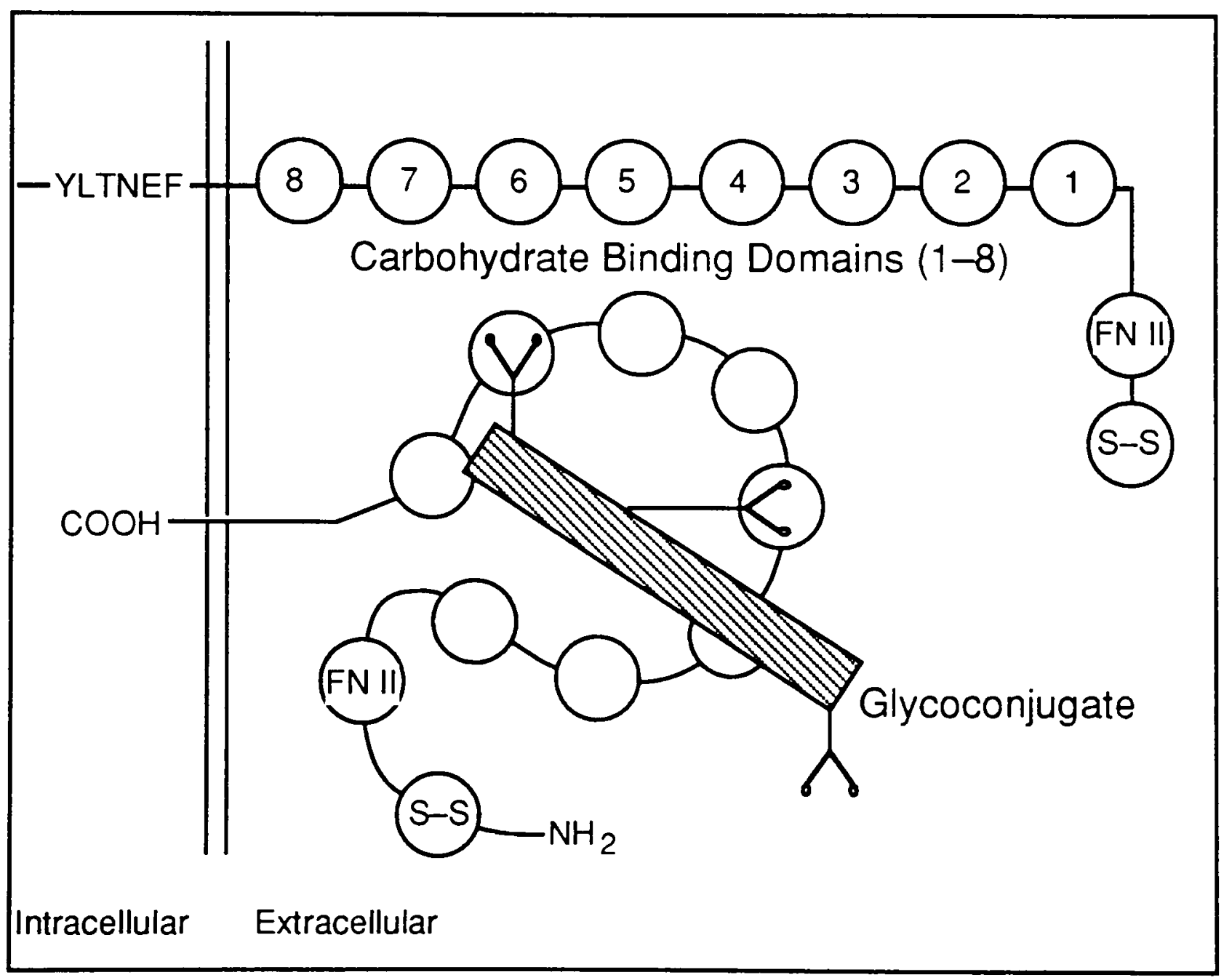

Fig. 5.6: Structure of the macrophage mannose receptor. The extracellular domain comprises a cysteine-rich region $(\mathrm{S}-\mathrm{S})$ followed by a region resembling the fibronectin type II domain (FN II) and eight repeats resembling carbohydrate recognition domains (CRD) of C-type lectins. The C-terminal cytoplasmic tail contains the sequence FENTLY for localisation in coated pits. Binding of multivalent ligand occurs through interactions with more than one CRD as shown. Adapted from reference [244]. 


\subsection{1 - Studies on the uptake of mannosylated BSA}

Many studies on the macrophage mannose receptor have made use of two highaffinity soluble ligands, $\beta$-glucoronidase and mannosylated bovine serum albumin (ManBSA). The latter neoglycoprotein is available commercially as a FITC-conjugate and was used to detect the presence of MR on Langerhans cells using a minor modification of the flow cytometric phagocytosis assay employed in previous chapters (see chapter 2 , section 2.5.4). A series of experiments was performed to determine whether FITCManBSA could be endocytosed specifically by Langerhans cells from C57Bl/6 mice. The uptake of FITC-ManBSA was saturable in a time-dependent (fig. 5.7a) and dosedependent fashion (fig. 5.7b) and was partly inhibitable by mannan at the longest time and highest dose tested (filled squares), indicating a receptor-mediated process. Incubation on ice gave no uptake (fig. 5.7a, open circle) and very limited binding.

a

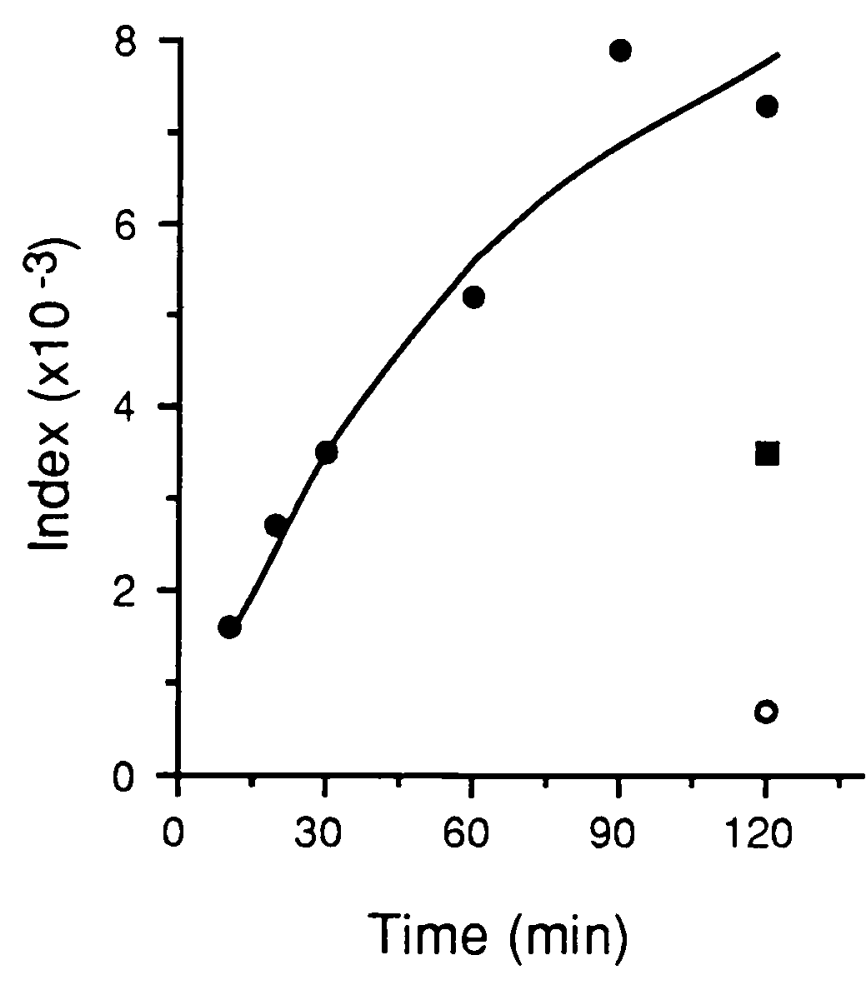

b

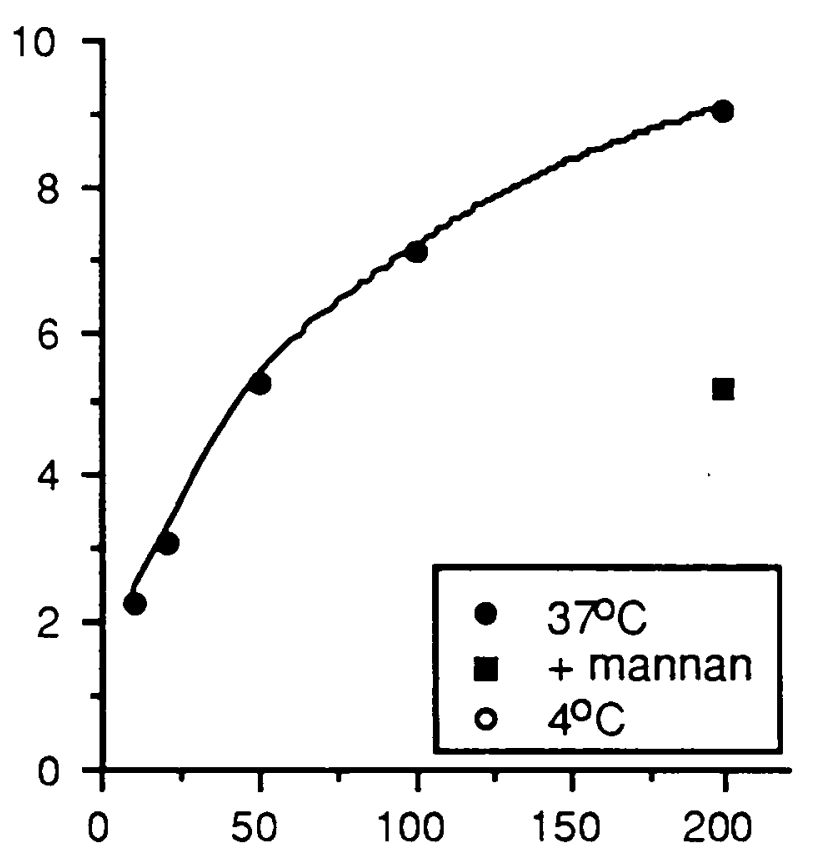

[FITC-ManBSA] $(\mu \mathrm{g} / \mathrm{ml})$

Fig. 5.7: FITC-ManBSA is endocytosed specifically by LC. EC from C57B1/6 cultured for $6 \mathrm{~h}$ were incubated with $100 \mu \mathrm{g} / \mathrm{ml}$ FITC-ManBSA for the times indicated (a) or with the indicated dose of the marker for $90 \mathrm{~min}(\mathrm{~b})$ at $37^{\circ} \mathrm{C}$. Where indicated the samples were incubated with excess mannan $(10 \mathrm{mg} / \mathrm{ml})$ or on ice. LC were analysed by FACS after staining for class II antigens. As in the phagocytic assays, index refers to the product of the percentage of LC positive for FITC by their mean fluorescence. a) and b) were obtained in separate experiments. Background cell fluorescence was subtracted in each case. 
To assess whether down regulation of the mannose receptor(s) recognising ManBSA occurred during LC maturation, a preliminary experiment was carried out to compare LC pulsed for $24 \mathrm{~h}$ immediately after isolation with those pulsed after culturing for $48 \mathrm{~h}$. Both C57Bl/6 and BALB/c LC endocytosed less FITC-ManBSA after culture (fig. 5.8a) whereas their capacity to endocytose FITC-BSA, a control marker that has no detectable sugar, either remained unchanged $(\mathrm{C} 57 \mathrm{Bl} / 6$; filled bars) or showed a slight increase (BALB/c; striped bars) (fig. 5.8b). Because the only difference between the two tracers is the presence of mannose residues on ManBSA this suggested that less mannose receptor was available for adsorptive endocytosis after LC culture.

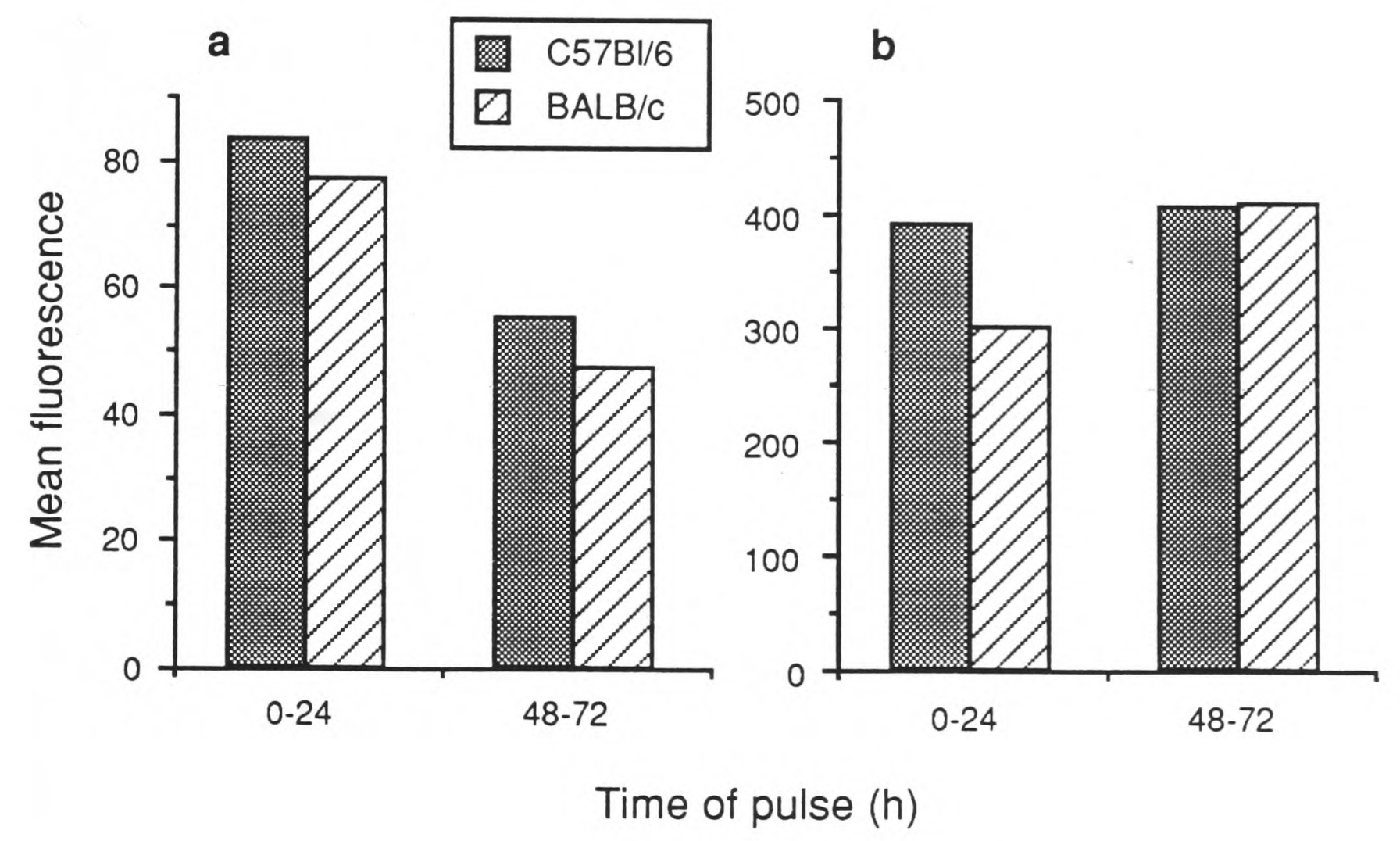

Fig. 5.8: Uptake of ManBSA is specifically down regulated during culture. $\mathrm{C} 57 \mathrm{Bl} / 6$ and BALB/c LC were incubated for $24 \mathrm{~h}$ with either a) FITC-Man BSA or b) FITC-BSA (both at $100 \mu \mathrm{g} / \mathrm{ml}$ ) at $0 \mathrm{~h}$ or $48 \mathrm{~h}$ of culture. Uptake by LC was measured by FACS and is expressed as the mean fluorescence of the whole LC population since, with soluble markers, there are no clear positive or negative cells. The mean fluorescence of controls incubated in medium alone was subtracted for each sample. Note that the absolute fluorescence values cannot be compared between a) and b) because of intrinsic differences in the fluorescence of FITC-ManBSA and FITC-BSA. 
The time course of ManBSA uptake during LC maturation was investigated using C57Bl/6 LC. Specific uptake (fig. 5.9a, triangles) followed a pattern very similar to that previously observed for zymosan (fig. 4.4), an initial rise followed by a decline (representative of three experiments; see also fig. 5.10a). Closer examination of this early rise (fig. 5.9b) showed that maximal uptake, after $6 \mathrm{~h}$ of culture, was greater than for cells incubated continuously from $0 \mathrm{~h}$ to $12 \mathrm{~h}$, possibly due to degradation of internalised ligand during the longer incubation, together with down regulation of uptake after 6-7h. 
a

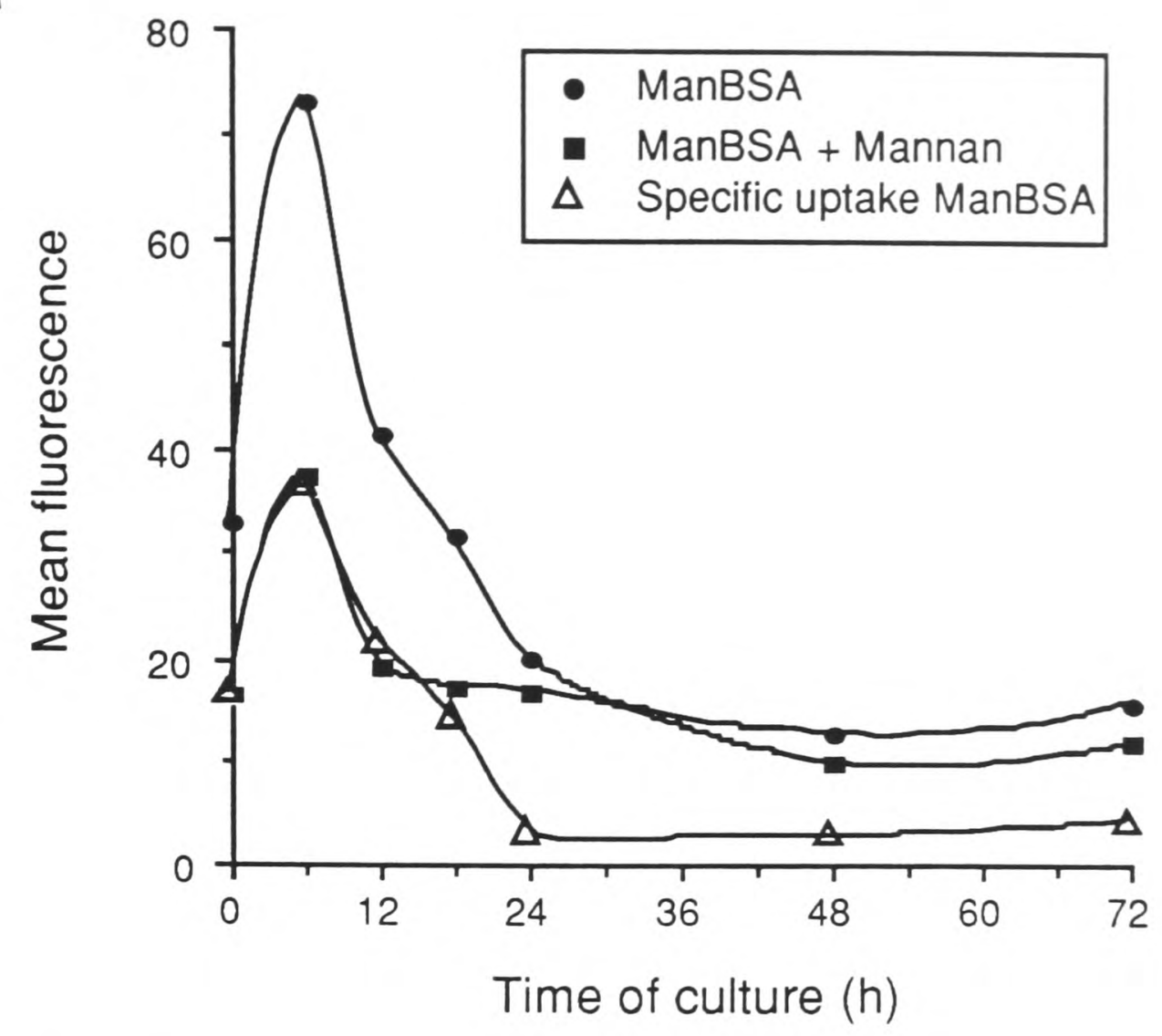

b

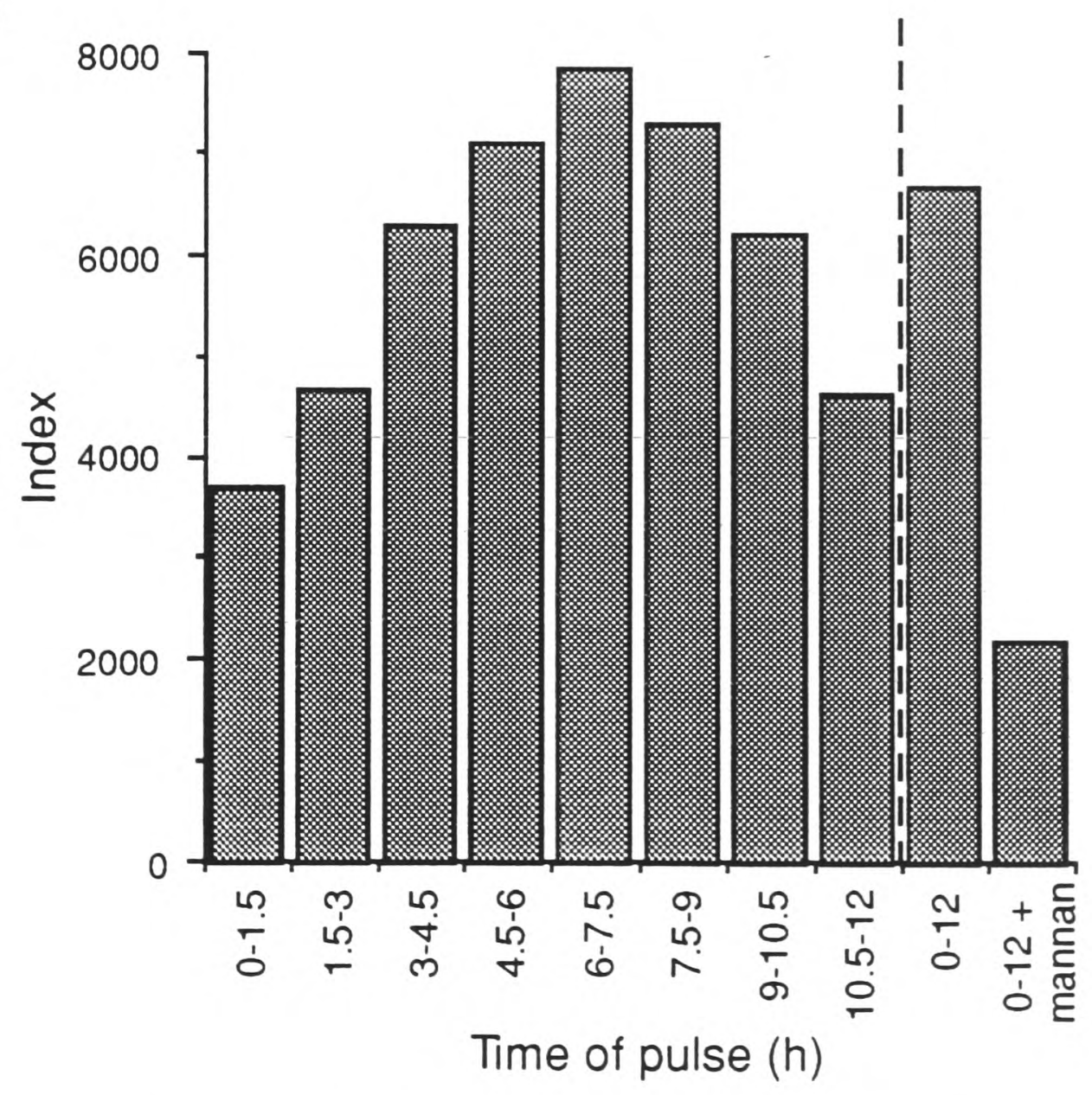

Fig. 5.9: Kinetics of uptake of ManBSA by C57Bl/6 LC. a) C57Bl/6 EC were pulsed for 90 min with FITC-ManBSA at the indicated culture times. FACS analysis of uptake by LC was performed as described in the methods and the mean fluorescence of controls was subtracted. Non-specific pinocytosis was controlled for by including samples incubated in the presence of excess mannan (10 mg/ml; squares). Specific uptake of ManBSA (triangles) was calculated by subtracting the values obtained with excess mannan from those ones obtained with FITC-ManBSA alone (circles) as in reference [255]. b) Uptake of FITC- 
ManBSA by LC was measured at 90 min intervals from $0-12 \mathrm{~h}$ of culture as in a) except that samples with mannan were not included for each time interval. Instead, a sample incubated continuously from $0-12 \mathrm{~h}$ of culture in the presence or absence of excess mannan acted as the control.

We conclude from these data that functional expression of the mannose receptor present in $\mathrm{C} 57 \mathrm{Bl} / 6 \mathrm{LC}$ is being specifically regulated during culture in a similar fashion to that previously observed for zymosan (fig. 4.4), that is, an initial rise followed by a decline. These conclusions are very similar to those reached in section 5.2.1 where zymosan uptake was inhibited by mannan.

The word "functional" has been carefully chosen, both in this and in the previous section. Functional expression is intended to mean the availability of receptor to exogenous ligand, i.e., how much ManBSA can be specifically internalised by mannose receptor (fig. 5.9a) or how much the uptake of zymosan can be inhibited by mannan (figs. 5.2-5.4). Whether there is a direct relationship between "functional" expression and physical expression of the receptor is a separate issue. In theory, it is possible to regulate the availability of MR to ligand simply by varying the rate with which it circulates through the endosomal compartment. Thus, the observation that the pinocytic rate in C57Bl/6 LC appeared to change during culture in a pattern like that observed for the specific uptake of ManBSA and zymosan is clearly worrying (fig. 5.10b). Pinocytosis of FITC-BSA increased by $6 \mathrm{~h}$ and subsequently decreased but was not affected by mannan, unlike the uptake of ManBSA (fig. 5.10a). 


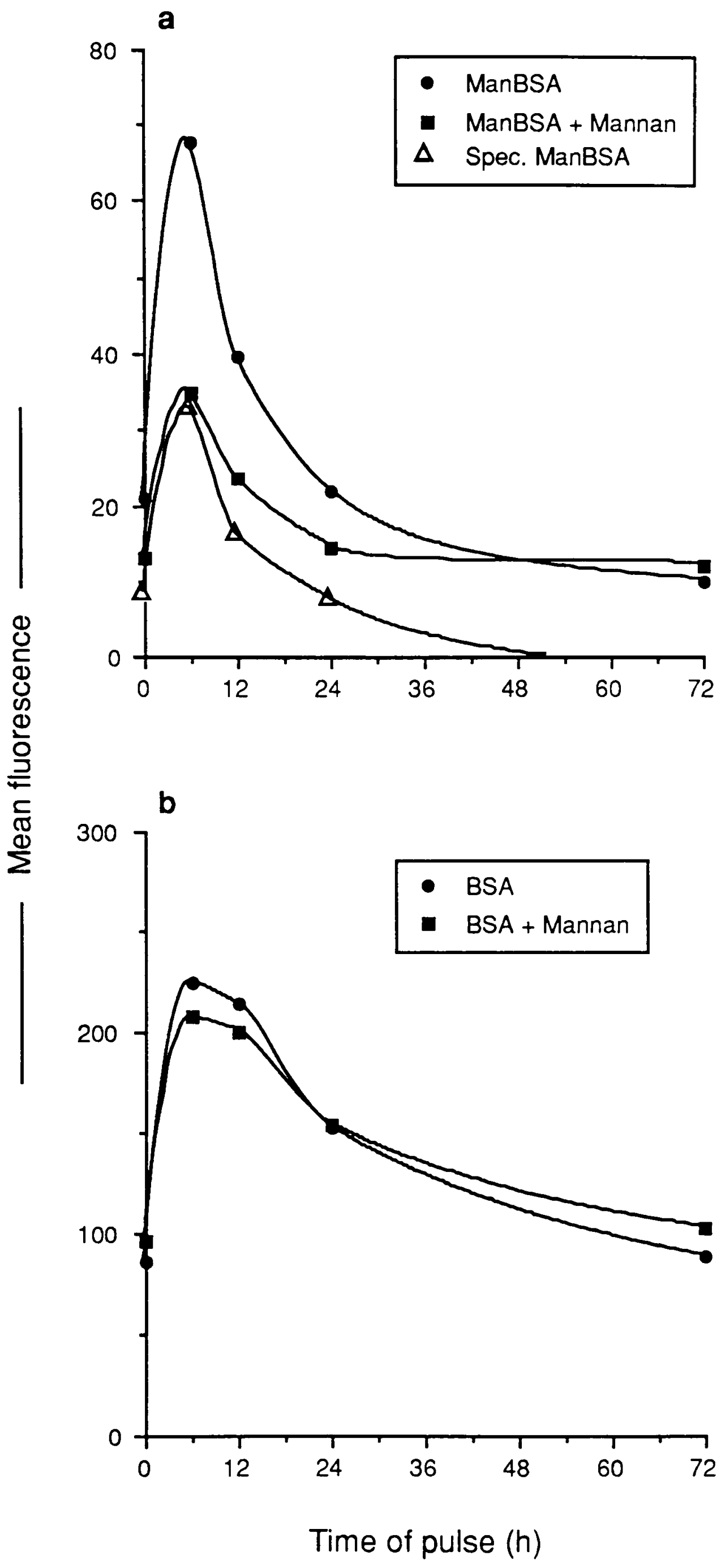

Fig. 5.10: Changes in the pinocytic rate of $\mathrm{C} 57 \mathrm{Bl} / 6 \mathrm{LC}$ accompany changes in the specific uptake of ManBSA. The experiment was performed exactly as in fig. 5.9a) using a) FITC-ManBSA or b) FITC-BSA as the endocytic tracers. 
The change in pinocytic rate could affect the rate of recycling from the early endocytic pathway $[255,256]$, making more or less receptor available for binding of ManBSA or zymosan during a 90 min incubation and thus leading to specific changes in uptake without regulation of receptor levels per se. Such reasoning could explain the increase in specific uptake of ManBSA at $6 \mathrm{~h}$ (figs. 5.7a and 5.8a, triangles). An increase in pinocytic rate, leads to more non-specific uptake (squares) but also more recycling of intracellular MR giving higher specific uptake (triangles). Very little information is available on endocytic traffic in LC and, at present, these hypotheses are difficult to test. Concomitant down regulation of receptor levels themselves is not excluded by these arguments adding a further dimension to the issue. A suggestion that it may be occurring comes from the observation that specific uptake of FITC-ManBSA was down regulated more abruptly after the 6h time point than that of FITC-BSA (compare fig. 5.10a and b). In an attempt to confirm that the pinocytic activity followed the pattern indicated by FITCBSA uptake, the experiment in fig. $5.10 \mathrm{~b}$ was repeated using a different pinocytic tracer, Lucifer yellow (LY). This tracer is an ideal probe for pinocytosis [258] and its excitation/emission spectrum [259] is just on the borderline of detection by the FITC channel of the FACScan (personal observations). The interpretation of results was complicated by technical problems (very few cells in some of the samples) but, again, a similar pattern of changes in endocytic traffic was obtained with LY as with FITC-BSA in fig. $5.10 \mathrm{~b}$ (data not shown).

\subsection{2 - Failure to find a fluorescent ligand to measure binding to MR rather than uptake}

Clearly, the problems discussed above would cease to apply if, instead of measuring uptake via MR one measured binding to the cell surface. Even if the pinocytic rate changed during culture, surface levels of MR should reflect those of the internal pool. ${ }^{4}$ Traditionally, this has been done for $\mathrm{M} \varnothing$ using binding of radioiodinated ManBSA to the

\footnotetext{
4 Provided that changes in the rate of pinocytosis are not disproportional to the rate of recycling; otherwise, the steady-state distribution of receptor, estimated at $80-90 \%$ intracellular in MØ [255, 256], could be affected.
} 
cell surface at $4^{\circ} \mathrm{C}$ in the presence or absence of a competing ligand. Such an approach, however, would require the use of purified LC since, in the course of the uptake studies described above, we noted that MHC class II-negative epidermal cells also endocytosed FITC-ManBSA (data not shown). To avoid purifying LC, we tried using FITC-ManBSA in a binding assay at $4^{\circ} \mathrm{C}$, analysing LC by double fluorescence flow cytometry as before. However, the signal obtained was very low and unreliable and results obtained in this way are shown only for the sake of completeness (fig. 5.11).

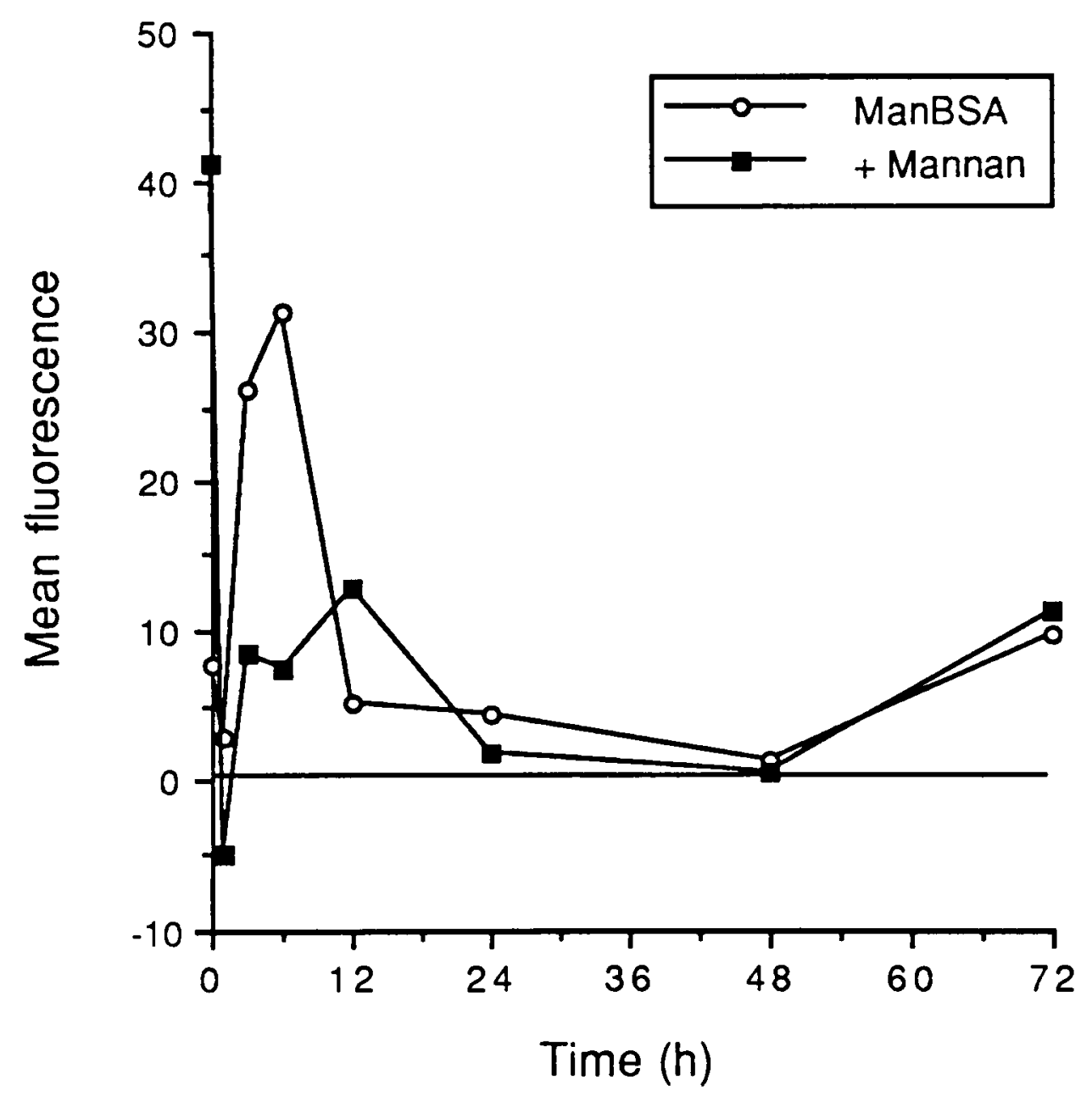

Fig. 5.11: Time course of binding of FITC-ManBSA to C57BI/6 LC. The experiment was performed as in fig. $5.11 \mathrm{a}$ except that samples were incubated on ice instead of $37^{\circ} \mathrm{C}$. The mean fluorescence of controls incubated in $\mathrm{R} 10$ alone has been subtracted at each time point. The FITC fluorescence of gated LC varied over a wide range. 
Approaches to try and amplify the signal using a rabbit anti-BSA antiserum, followed by FITC-conjugated donkey anti-rabbit were unsuccessful. One of the problems associated with the use of FITC-ManBSA in binding studies appeared to be a high background, possibly due to affinity of the hydrophobic FITC moiety for cell membranes. The lack of a positive signal above background may also occur if MR is primarily located in an intracellular endosomal pool in LC, as in MØ. It was later confirmed that even with J774E MØ, which have been selected for high MR expression [218], FITC-ManBSA gave little specific signal in binding assays although it was specific in uptake assays (not shown). As an alternative MR ligand, DNP- $\beta$-glucuronidase was tested on J774E cells and visualised with a mouse anti-DNP monoclonal followed by a FITC-conjugated antimouse reagent. Again, binding was not inhibitable by mannan (not shown) and this ligand was not pursued further.

Because of the intracellular distribution of MR in MØ, permeabilisation of the cells allows access to the endosomal pool and makes a much larger number of receptor molecules available to bind ligand [256]. We followed up this idea by adapting a permeabilisation procedure developed for FACS analysis of intracellular antigens ([219]; see chapter 2, section 2.4.5) and testing it on J774E MØ. However binding of both FITCManBSA and FITC-mannan were non-specific (not shown). A final attempt at finding a suitable ligand, in the form of mannan-coated fluorescent latex beads, also proved unsuccessful because association with cells was largely non-specific. We conclude that it is not feasible to assay ligand binding to MR by flow cytometry at the present time.

\section{4 - Biochemical analysis of mannose receptor expression by LC and MØ}

Studies with ManBSA did not allow us to determine whether the activity detected in fresh LC was due to macrophage mannose receptor expression by these cells or due to a different receptor, also capable of binding mannose residues. Clearly, much more information about mannose receptor activity in Langerhans cells could be gained from 
access to antibodies against murine MR. Therefore, in collaboration with Prof. P.D. Stahl of Washington University, St. Louis, MO, U.S.A., studies were performed with a new polyclonal rabbit antiserum raised against murine mannose receptor purified from J774E cells. The characterisation of this new reagent was performed by the author and is described below.

\subsection{1 - Characterisation of a rabbit antiserum against murine macrophage mannose receptor}

The isolation and characterisation of J774E mannose receptor have been described [260]. MR kindly purified from these cells by Mrs. E. Peters, Dept. of Cell Biology and Physiology, Washington University, was used to immunise a rabbit. Antiserum was collected by exsanguination following two boosts, after positive test bleeds indicated the presence of specific reactivity. By immunoprecipitation and Western blotting, the antiserum was found to identify a single major band (fig. 5.12) and, in a separate immunoprecipitation and Western blot, the same band was identified by a goat antiserum against human MR that cross-reacts with mouse (not shown). A lower molecular weight, fainter band was often seen in conjunction with the main receptor band (fig. 5.12) and represents newly-synthesised receptor not yet modified by glycosylation (reference [261] and S. Pontow and P. D. Stahl, personal communication). The molecular weight of the mature protein recognised by the antiserum fell in the range $165-180 \mathrm{kD}$, in accordance with published data $[260-264]$, and the antibody did not recognise reduced antigen (fig. 5.13). 
a

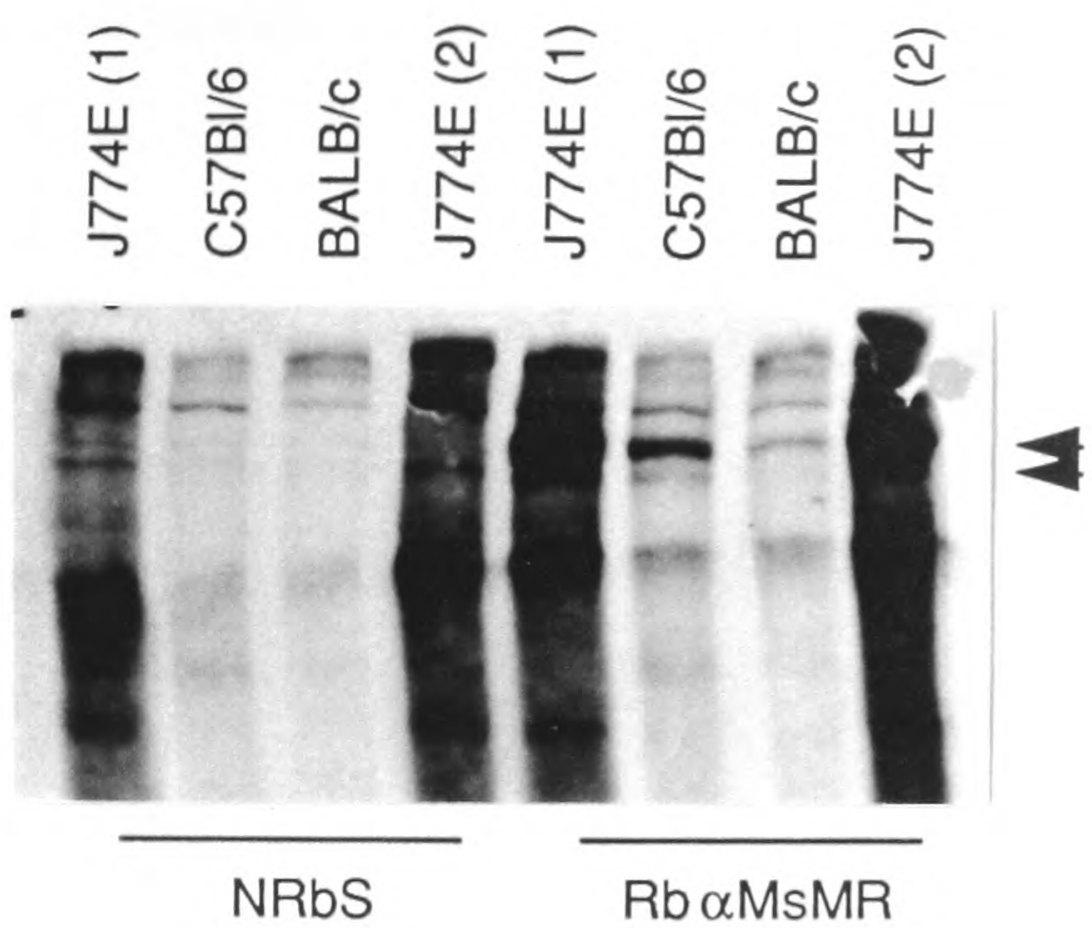

b

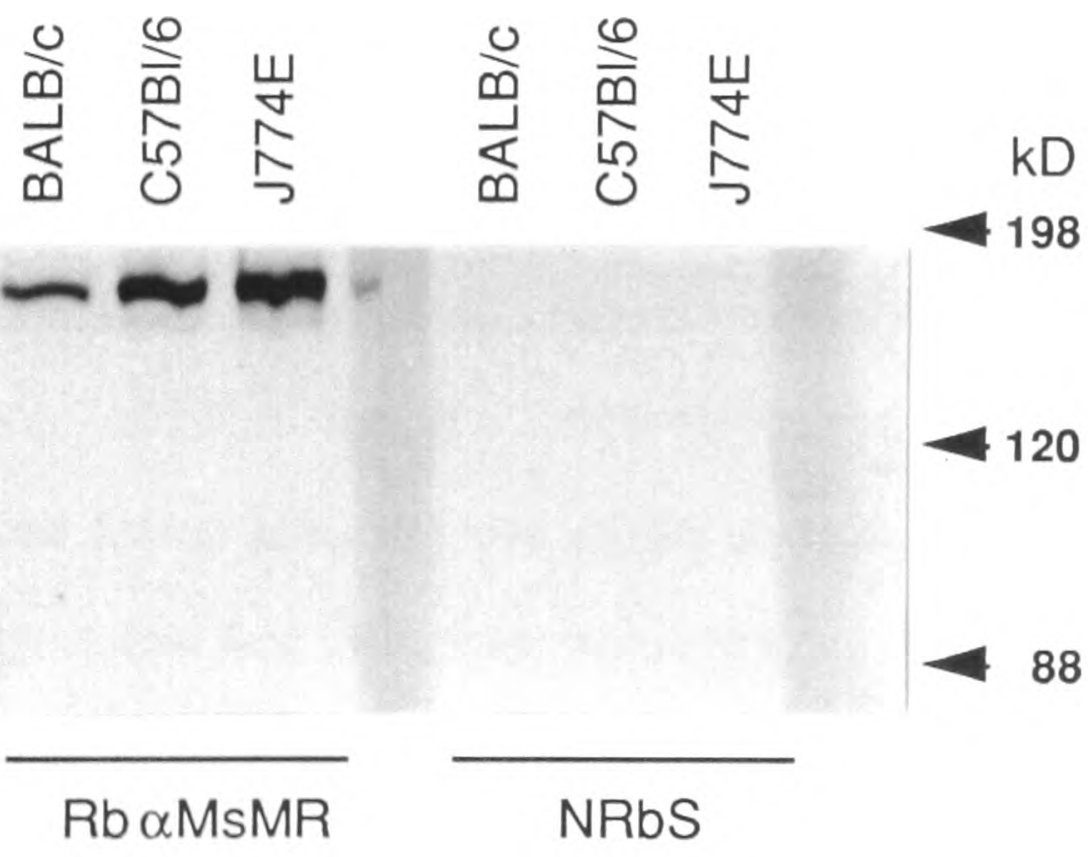

Fig. 5.12: Novel rabbit antiserum against murine mannose receptor. Rabbit antiserum against murine mannose receptor (RboMsMR) was evaluated by immunoprecipitation (a) and Western blotting (b) of total MØ protein; normal rabbit serum (NRbS) was used as a control. Cells were thio-M $\varnothing$ from $\mathrm{C} 57 \mathrm{Bl} / 6$ and $\mathrm{BALB} / \mathrm{c}$ mice or $\mathrm{J} 774 \mathrm{E}$, as indicated above each lane. J774E (1) and J774E (2) denote lysates from two separate cultures. The same amount of total cellular protein was used as the starting material for immunoprecipitation (a) or was loaded onto each lane for the Western blot (b). Arrowheads in a) indicate the receptor bands; ${ }^{14} \mathrm{C}$ molecular weight markers in a) were not detectable on the autoradiograph because of the short exposure time. Molecular weights indicated in $b$ ) reflect the position of pre-stained standards and are only approximate. 
Surprisingly, less receptor was detected in $\mathrm{BALB} / \mathrm{C} \mathrm{M} \varnothing$ lysate than in $\mathrm{C} 57 \mathrm{Bl} / 6$ or J774E lysates by Western blotting (fig. 5.12b); BALB/c also appeared to lack the unprocessed receptor band (fig. 5.12b). Similarly, less receptor was immunoprecipitated from $\mathrm{BALB} / \mathrm{c} \mathrm{M} \varnothing$ than from $\mathrm{C} 57 \mathrm{Bl} / 6 \mathrm{M} \varnothing$ or $\mathrm{J} 774 \mathrm{E}$ cells in spite of the fact that the same amount of cellular protein was used as the starting material for precipitation (fig. 5.12a). Again, in the immunoprecipitation, the band corresponding to unprocessed receptor appeared to be absent from BALB/c M $\varnothing$ (fig. 5.12a). However, it was detected when the autoradiograph was overexposed (not shown) and, therefore, the apparent absence is probably due to less receptor overall being present in BALB/c M $\varnothing$ (although we cannot exclude the possibility that differential rates of processing in the two strains are also responsible). The experiment was repeated once with similar results but the strain difference was less noticeable than previously (fig. 5.13; notice band for unprocessed receptor in $\mathrm{C} 57 \mathrm{Bl} / 6$ but not in $\mathrm{BALB} / \mathrm{c}$ lanes and the difference in intensity of the processed receptor band in the two strains). The two experiments shown in fig. 5.12 and 5.11 were done in the U.S.A and in the U.K., respectively, using mice from different sources and making it very unlikely that the strain difference observed was due to a peculiar infection or other factor affecting one of the strains. These results were not pursued further but suggest that less MR may be expressed in BALB/c compared to $\mathrm{C} 57 \mathrm{Bl} / 6 \mathrm{M} \varnothing$. Similar conclusions were suggested for LC from the data on the extent of inhibition of zymosan uptake by mannan (section 5.2.1). 


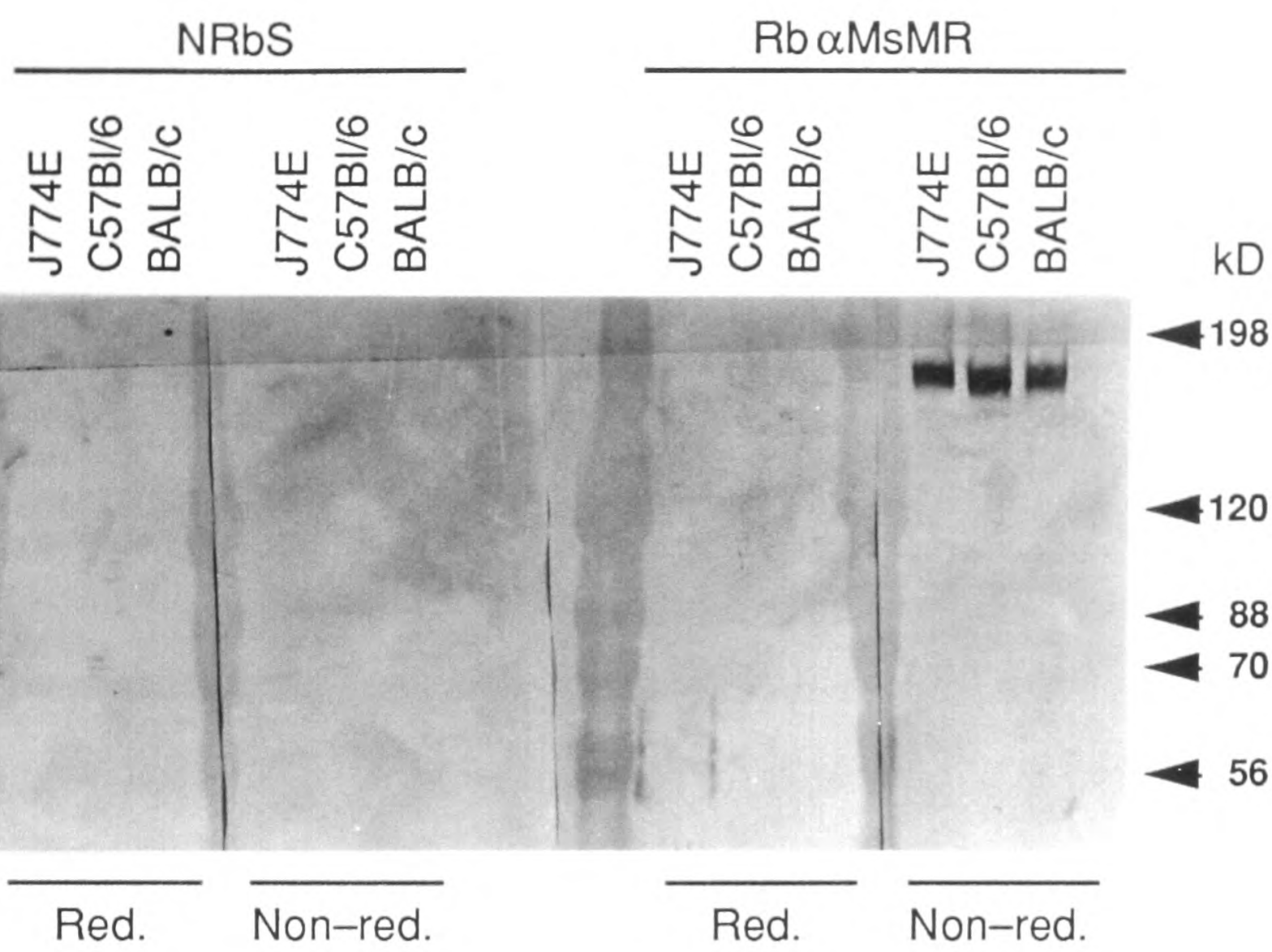

Fig. 5.13: Mannose receptor expression by macrophages. $2.4 \mu \mathrm{g}$ of protein from total cell lysate of thio-M $\varnothing$ or J774E cells were run on a $7.5 \%$ polyacrylamide resolving gel under reducing (red.) or non-reducing (non-red.) conditions. The proteins were transferred to nitrocellulose membranes and probed with anti-mannose receptor antiserum or normal rabbit serum as in fig. 5.12. Indicated molecular weights reflect the position of pre-stained standards and are only approximate. The thin line across the top of the blot is the edge of sticky tape used to paste the nitrocellulose strips together after probing. Notice that antimurine MR antiserum does not recognise reduced antigen.

5.4 .2 - Anti-mouse MR antiserum does not inhibit receptor-mediated uptake of $M R$ ligands and is a poor reagent for immunofluorescence

Preliminary experiments were carried out with J774E M $\varnothing$ to assess the potential usefulness of the new anti-murine MR antiserum in functional studies, eventually to be applied to LC. However, attempts to inhibit binding or internalisation of various ligands for MR failed consistently. The results of one such experiment are shown in table 5.6. Heat inactivated (HI) antiserum did not inhibit uptake of FITC-conjugated zymosan, mannan or 
ManBSA by J774E macrophages. In contrast, purified mannan was able to inhibit uptake/binding of zymosan by about 30\%, inhibited uptake of ManBSA (35\%) without affecting uptake of BSA and competed efficiently with FITC-mannan, reducing its uptake by $73 \%$. Dextran was used as a control glycan and gave a small degree of inhibition in this experiment but not in many others. Note that highly-purified $\beta$-glucan was an efficient inhibitor of zymosan uptake, in accordance with reports that murine macrophages possess a $\beta$-glucan receptor [237] and in contrast to the data presented in section 5.2.2. The lack of inhibition achieved with anti-murine MR antiserum cannot be attributed to heat treatment since heat-inactivated antiserum could immunoprecipitate and immunoblot MR and antiserum that was not inactivated gave similar results to the ones in table 5.6 (not shown). Rather, the results in table 5.6 suggest that the antiserum does not contain antibodies against the receptor binding sites because a) the binding sites are very poorly immunogenic and/or b) the receptor binding sites are highly conserved between mouse and rabbit.

Table 5.6: Rabbit anti-mouse mannose receptor does not inhibit internalisation via MR

\begin{tabular}{cccccc}
\hline & & FITC- & FITC- & FITC- & \\
Inhibitor & Conc. & zymosan & Mannan & ManBSA & FITC-BSA \\
\hline Control & -- & 100.00 & 100.00 & 100.00 & 100.00 \\
Mannan & $1 \mathrm{mg} / \mathrm{ml}$ & 71.06 & 26.93 & 64.21 & 95.68 \\
$\beta$-glucan & $1 \mathrm{mg} / \mathrm{ml}$ & 48.78 & 78.17 & 98.89 & 107.48 \\
Dextran & $1 \mathrm{mg} / \mathrm{ml}$ & 88.16 & 82.24 & 91.01 & 100.54 \\
HI Rb anti-Ms $M R$ & $1: 500$ & 91.25 & 100.12 & 90.48 & 92.53 \\
HI Rb anti-Ms $M R$ & $1: 1000$ & 84.70 & 92.69 & 87.84 & 93.20 \\
HI Rb anti-Ms $M R$ & $1: 2000$ & 86.44 & 101.78 & 90.43 & 97.92 \\
HI NRbS & $1: 500$ & 89.19 & 99.92 & 98.97 & 101.62 \\
HI NRbS & $1: 1000$ & 81.30 & 101.97 & 93.49 & 102.12 \\
HI NRbS & $1: 2000$ & 82.46 & -- & 86.92 & 90.78 \\
\hline
\end{tabular}

$\mathrm{J} 774 \mathrm{E} \mathrm{M} \varnothing$ were pre-incubated with the indicated inhibitors and controls for $30 \mathrm{~min}$ at $37^{\circ} \mathrm{C}$ before adding FITC-zymosan $(20 \mu \mathrm{g})$, FITC-mannan, FITC-ManBSA or FITCBSA (all at $10 \mu \mathrm{g} / \mathrm{ml}$ ) for a further $90 \mathrm{~min}$. Uptake was assayed by FACS using single colour analysis. Results are expressed as a percentage of control samples without inhibitor. Results showing lack of inhibition by anti-mouse MR antiserum are italicised. HI, heat inactivation $\left(20 \mathrm{~min}\right.$ at $\left.56^{\circ} \mathrm{C}\right)$ was used to inactivate complement thereby enabling the antiserum to be used on live cells. 
It was hoped that the new anti-murine MR antiserum could be used in immunofluorescence studies to establish whether the mannan-inhibitable receptor in LC was the same mannose receptor expressed by $M \emptyset$. Microscopical observation of permeabilised J774E M $\varnothing$ stained with the antiserum revealed a punctate pattern reminiscent of endosomal staining and which was not present in control samples (not shown). However, background fluorescence was always very high and the intensity of staining was not above that of the control $(\mathrm{NRbS})$. The high background fluorescence precluded FACS analysis; in over 10 experiments on J774E cells, trying different FITCconjugated anti-rabbit reagents, diverse $\mathrm{Fc}$ receptor blocking procedures, several amplification methods and using cell permeabilisation techniques to allow access to the intracellular receptor pool, the signal detected by FACS was, consistently, barely above background. The results of one such experiment are shown on table 5.7. To obviate the background fluorescence problem, a $F\left(a b^{\prime}\right)_{2}$ fragment of IgG from the whole antiserum was made (see chapter 2, section 2.8.6) and compared to controls from normal rabbit. This approach was successful in reducing background fluorescence due to rabbit serum (table 5.7; compare mean fluorescence of NRbIgG and NRbIgG F(ab') 2 with $\mathrm{HI}$ NRbSerum) but also reduced the signal obtained with the antiserum such that the signal to noise ratio was reduced in permeabilised cells. There was a slight increase in the signal to noise ratio of intact cells consistent with a decrease in Fc receptor-mediated non-specific binding to the cell surface (table 5.7). The decrease in signal may be due to loss of activity of the antiserum during the preparation of the $F\left(a b^{\prime}\right)_{2}$ fragment but, more likely, represents decreased binding of the second stage reagent, FITC-donkey anti-rabbit, which was directed against whole rabbit IgG. Use of a FITC-conjugated goat antiserum specific for rabbit $\mathrm{F}(\mathrm{ab})_{2}$ did not improve staining, however (not shown). Note that, although whole antiserum gave a signal to noise ratio of 2.23 in permeabilised J774E cells (table 5.7), since fluorescence is displayed on a logarithmic scale, there is only a small visual shift in the fluorescence peaks between the positive and negative samples (not shown). In spite of this shortcoming we attempted to analyse permeabilised LC to see if this small shift in peak could be detected. The results were inconclusive, a shift in peak being observed in some 
samples but not in others (not shown). We conclude that it is not feasible to use our rabbit anti-mouse mannose receptor antiserum for immunofluorescence.

Table 5.7: Anti-murine mannose receptor cannot be used for immunofluorescence

\begin{tabular}{lcc}
\hline & \multicolumn{2}{c}{ Mean fluorescence } \\
\cline { 2 - 3 } \multicolumn{1}{c}{ Antibodies } & Intact & Permeabilized \\
\hline NRb IgG & 16.07 & 40.77 \\
Anti-Ms MR IgG & 17.97 & 48.47 \\
NRb IgG F(ab')2 & 6.74 & 11.31 \\
Anti-Ms MR IgG F(ab') 2 & 8.35 & 16.76 \\
HI NRbSerum & 33.86 & 49.16 \\
HI Anti-Ms MR serum & 36.43 & 109.39 \\
None & 5.62 & 6.73
\end{tabular}

\begin{tabular}{lll} 
Anti-Ms MR IgG & 1.12 & 1.19 \\
Anti-Ms MR IgG F(ab')2 & 1.24 & 1.48 \\
HI Anti-Ms MR serum & 1.08 & 2.23 \\
\hline
\end{tabular}

Intact or permeabilised, fixed J774E M $\varnothing$ were reacted with polyclonal rabbit anti-mouse MR in the form of whole heat-inactivated serum, purified $\operatorname{IgG}$ or $F\left(a b^{\prime}\right)_{2}$ fragment or the respective forms of a control polyclonal from a normal rabbit. Bound antibody was detected using a FITCconjugated donkey anti-rabbit polyclonal. Mean fluorescence values were obtained by FACS analysis. Signal/noise ratios were calculated by dividing the mean fluorescence of positive samples by that of the appropriate controls. Two additional dilutions of each antibody were included in this experiment with the same results but are omitted for the sake of simplicity.

\subsection{3 - Analysis of MR expression in LC by Western blotting}

Since the anti-mouse MR antiserum proved to be such a good reagent for biochemical analysis of $M \varnothing$ protein (section 5.4.1) we used Western blotting of lysates 
from Langerhans cells to attempt to determine the potential expression of MR in these cells. Western blotting is a simple and a powerful technique provided one has a readily available source of protein. However, purification of LC in sufficient numbers to make protein analysis feasible is not a trivial task. Before attempting to purify LC for protein analysis we carried out pilot experiments to determine the minimum amount of protein that could be loaded per lane and still result in detection. The signal from J774E protein was not titrated out at $0.6 \mu \mathrm{g} / \mathrm{lane}$ although it was very weak; however, it was easily detectable at $1.2 \mu \mathrm{g} / \mathrm{lane}$ (not shown; see also fig. 5.14). We also ascertained that no signal was detectable in whole epidermal cell suspensions to ensure that any contaminants in purified LC preparations did not affect the outcome of the experiment (data not shown and fig. 5.14).

Two separate experiments were carried out with $6 \mathrm{~h}$-cultured C57Bl/6 LC purified using the B21.2 antibody against MHC class II antigens and magnetic beads coated with goat anti-rat (see chapter 2, section 2.3.4). The yield of LC in both experiments was very low ( $<5 \times 10^{4}$ cells) and calculation of the protein concentration in lysates had to be adjusted for protein eluted from the magnetic beads. Thus, in one experiment (fig. 5.14), beads alone in lysis buffer gave $8.0 \mu \mathrm{g}$ protein whereas the lysate of the bead-attached LC fraction $\left(\mathrm{B} 21.2^{+}\right)$contained $10.8 \mu \mathrm{g}$ protein. By subtraction, the cell-derived protein was $2.8 \mu \mathrm{g}$ and this was split between two lanes giving $1.4 \mu \mathrm{g} \mathrm{LC}$ protein/lane (fig. 5.14). Control lanes were loaded with protein from a lysate of $\mathrm{C} 57 \mathrm{~B} 1 / 6$ thio-M $\varnothing$ : the same

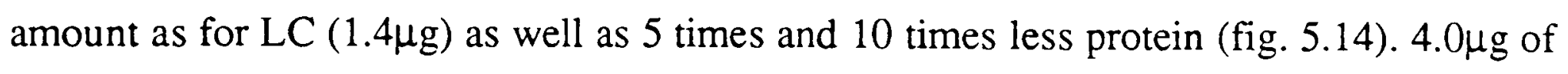
bead protein (the same amount of bead protein as that contaminating the LC lysate) and

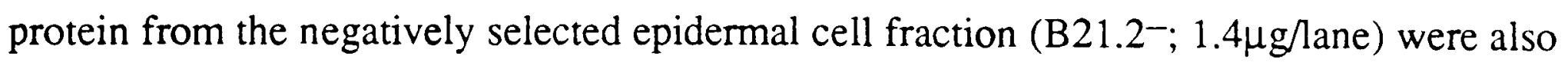
included. The results (fig. 5.14) demonstrate that a mannose receptor-specific band was not detectable in the LC or the LC-depleted EC fraction. In the M $\varnothing$ control lanes a band was detectable at $0.3 \mu \mathrm{g}$ total cell lysate/lane and was still not completely titrated out at $0.15 \mu \mathrm{g} /$ lane. The band at $\approx 55 \mathrm{kD}$ in the $\mathrm{LC}$ fraction is due to cross-reactivity of the antiserum with protein from the magnetic beads (fig. 5.14, lane "beads"). Cross-reaction 
appears to be with FCS protein passively adsorbed onto the beads since it is also seen with uncoated magnetic beads incubated in R10 (not shown).

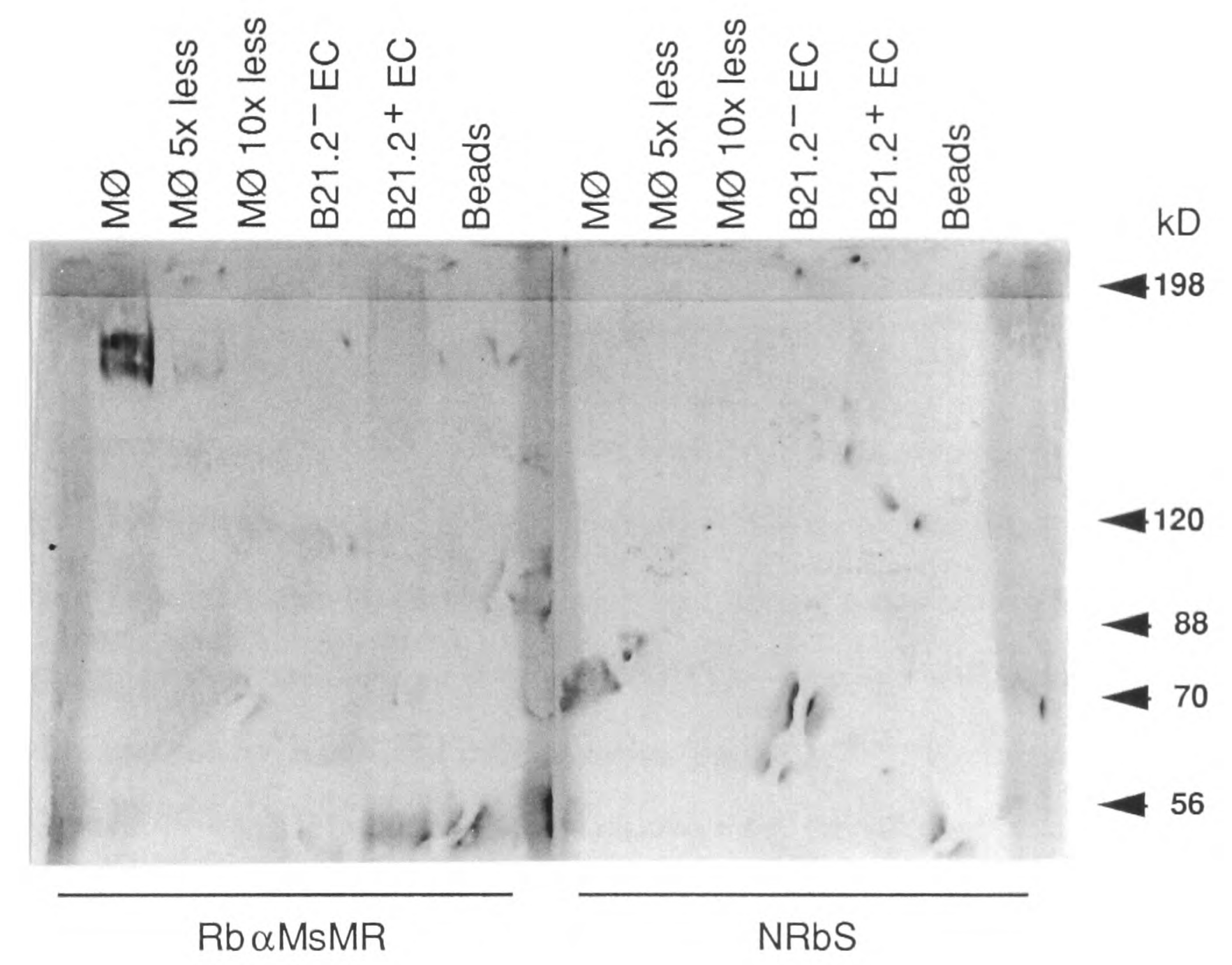

Fig. 5.14: Macrophage mannose receptor is not detectable in LC purified by immunomagnetic means. 6h-cultured LC from $30 \mathrm{C} 57 \mathrm{Bl} / 6$ mice were purified using B21.2-coated magnetic beads as described in section 2.3.4. Lysates were run on a 7.5\% SDS-polyacrylamide gel, transferred to nitrocellulose and probed with anti-mouse MR antiserum or normal rabbit serum as a control. See text for details. Similar results were obtained in a separate experiment. The thin line across the top of the blot is the edge of sticky tape used to paste the nitrocellulose strips together after probing.

The negative results shown on fig. 5.14 and similar results obtained in a separate experiment should not be taken to suggest that the macrophage mannose receptor is not expressed by LC. The subtraction method for determining LC-derived protein is unreliable 
when such small quantities of LC protein are involved and because more bead-derived than LC-derived protein was present in bead-associated LC lysate. It is, therefore. probable that the LC-derived protein constituted an insignificant percentage of the protein in the lysate. Note that, had a positive result been obtained, these criticisms would not apply. The presence of a band corresponding to mannose receptor in LC lysate would have meant that these cells express the protein; the absence of such a band is merely inconclusive. In retrospect, it would have been appropriate to re-probe the blot with an antibody against a LC marker, such as class II, to ascertain the presence of LC protein. This was carried out for a similar experiment (see below).

Attempts to purify LC by cell sorting resulted in very pure populations but, again, extremely low yields of LC, making protein analysis unfeasible (not shown). An alternative approach was to purify the cells by panning on antibody-coated dishes. A modification of conventional panning procedures - "mismatched" panning — has been used with success by Koch and colleagues to purify LC [140]. We made use of this technique as follows: LC in fresh EC suspensions were pre-enriched to 10-28\% [140] by killing many keratinocytes and all $\gamma / \delta \mathrm{T}$ cells with anti-Thy-1 and complement; the preenriched population was labelled with rat anti-Ia (B21.2) and rat anti-CD45 (TIB 122) and panned on dishes coated with goat anti-mouse polyclonal. Because the polyclonal crossreacts with rat antibodies, LC were panned successfully and were easily recovered afterwards by adding an excess of species-matched (mouse) IgG. The yield of fresh (6hcultured) LC was 10-fold higher than that obtained with magnetic beads in one preparation $\left(2 \times 10^{5}\right.$ cells $)$ and their purity was estimated at $70 \%$. Lysate from these cells was compared to lysate from $\mathrm{C} 57 \mathrm{Bl} / 6$ thio- $\mathrm{M} \varnothing$ and lysate from three day cultured low density $\mathrm{EC}$ (containing $\approx 14 \% \mathrm{LC}$ ). The concentration of protein loaded onto the gel was adjusted to compensate for the differences in percentage of LC in each population. Qualitatively similar results to those in fig. 5.14 were obtained (not shown). However, when the blot was re-probed with B21.2 as a control for the presence of LC protein, 6h LC failed to show a band for class II although a band was readily detectable in day 3 low density EC lysate (not 
shown). Thus, once more, the results for fresh LC should be regarded as inconclusive although it does appear that MR is not present in cultured LC.

These results are currently being pursued but cannot be included here because of time constraints. The preliminary results presented in this section do not allow us to conclude that macrophage mannose receptor is not expressed by LC. 


\section{5 - Summary}

This chapter has shown that uptake of zymosan by fresh LC can be specifically inhibited by mannan and $\beta$-glucan but not by a control polysaccharide, dextran (figs. 5.2, 5.3), or, apparently, by antibodies against CR3 (section 5.2.2). These observations suggest that fresh LC phagocytose zymosan through recognition units for $\beta$-glucan and mannose, similar to the receptors involved in uptake of zymosan by mononuclear phagocytes and neutrophils (see table 5.1) although the possibility that a novel receptor(s) is expressed by LC is not excluded. Inhibition by mannan and $\beta$-glucan acted preferentially on C57Bl/6 LC suggesting that LC of this strain expressed higher levels of the relevant receptors and correlated with the observation that they were more phagocytic for zymosan than LC from $\mathrm{BALB} / \mathrm{c}$ mice (figs. 5.3, 5.4). Interestingly, lysates of thioglycollate-induced M $\varnothing$ from C57B1/6 mice contained higher levels of mannose receptor than those from BALB/c (figs. $5.12,5.13$ ) but no strain differences in the uptake of zymosan were ever noted for these cells (table 5.2). Inhibition of LC uptake by both mannan and $\beta$-glucan was lost during culture, consistently with the hypothesis that the receptors involved were being functionally down regulated, concomitantly with phagocytic activity (figs. 5.4, 5.5). For receptor(s) recognising mannose-containing ligands, the observation that specific uptake of FITC-ManBSA by C57Bl/6 LC decreased in culture with kinetics similar to those of zymosan uptake (figs. 5.8-5.10) provided further evidence for functional down regulation. The observation that the uptake of pinocytic tracers changed in a like fashion raised the question of whether the receptor for mannose was physically down regulated during LC maturation or preferentially sequestered intracellularly in mature cells (fig. 5.10). To address this question, and to establish whether the LC receptor for mannose was the macrophage mannose receptor, we tested a novel rabbit antiserum against murine MR. Unfortunately, we were unable to use this reagent in inhibition or immunofluorescence assays (tables $5.6,5.7$ ) and analysis of total LC protein proved to be inconclusive because of the difficulty in purifying these cells (fig. 5.14). 


\section{Chapter 6}

\section{Summary and Discussion}

6.1 - Summary

6.2 - Technical considerations

6.3 - Phagocytosis of antigens by dendritic cells

6.4 - Receptors mediating phagocytosis by Langerhans cells

6.5 - The relevance of phagocytosis by Langerhans cells 


\section{1 - Summary}

Presentation of exogenous antigens requires their internalisation and processing in endolysosomal compartments, and expression at the cell surface in the form of peptideMHC class II complexes. For the initiation of primary adaptive immune responses against pathogenic organisms, because of the specialised immunostimulatory properties of dendritic cells, it would seem essential that members of this lineage should, at some stage, be capable of phagocytosis. In this thesis we have demonstrated that freshly-isolated LC can phagocytose a variety of particles, ranging in size from $0.5-6 \mu \mathrm{m}$, and down regulate this ability during maturation in culture. Shutdown of MHC class II and invariant chain synthesis and loss of acidic vesicles from the endocytic pathway have been observed during maturation of LC in vitro (see chapter 1) but, to our knowledge, this is the first report that maturation of $\mathrm{LC}$ also involves down regulation of the initial step in antigen presentation, namely the capacity to internalise antigens. Freshly-isolated splenic DC had moderate phagocytic activity, in line with recent observations that, at least some, freshlyisolated splenic DC are immature, but underwent a similar transformation to LC into nonphagocytic cells after overnight culture. We investigated some of the receptors involved in phagocytosis by LC and we attempted to correlate their functional expression with changes in phagocytic activity. The presence of receptors recognising mannose and $\beta$-glucan residues in fresh LC was implicated by inhibition studies on the uptake of zymosan, a yeast-derived particle, and by studies with ManBSA, a ligand for MR. In contrast to these glycan receptors, which have not been previously described in DC, Fc $\gamma$ and complement receptors are expressed by these cells but did not appear to be active in mediating particle uptake, although it is possible that CR3 co-operates in the phagocytic process through interactions with other receptors or, in the case of opsonised particles (e.g. C. parvum), through direct interactions with the target. In this chapter we summarise these findings and discuss their implications. 


\section{2 - Technical considerations}

It is easy to be complacent about the limitations of work on Langerhans cells and we feel they ought to be justified in this discussion. Murine epidermal Langerhans cells are traditionally obtained from mouse ears, this being the area of skin most amenable to manipulation. 20-30 mice were needed in order to carry out a typical flow cytometric experiment. In experiments where two strains were compared, such as that shown in fig. 4.4 , for example, 60 mice were used (30 of each strain). The workload and time involved in peeling 120 ears and separating the epidermis from 240 ear-halves is considerable. The monetary cost and the duration of most experiments $(0-72 \mathrm{~h}$ culture, followed by cell staining) meant a maximum of 1-2 experiments could be performed each week. FACS analysis, itself, was often a lengthy procedure since acquisition of each sample of gated LC took up to $15 \mathrm{~min}^{1}$, such that analysis of an experiment such as that shown in fig. 4.4 required over $6 \mathrm{~h}$ of continuous flow cytometry. Thus, the conspicuous absence of error bars from all our data reflects the fact that it was not feasible to include replicates within an experiment. The validity of our results was, instead, checked by the reproducibility of our findings: virtually all of the experiments presented in this thesis were done at least twice and most were performed 3-5 times, with similar results unless noted. The severe limitation imposed by the availability of cells is also manifest in the design of the experiments. For example, in fig. $5.9 \mathrm{~b}$ one would, ideally, have liked to include samples with excess mannan for each time interval to control for non-specific uptake. However, the number of cells available at the outset of the experiment meant that only one such control sample was available; we chose to incubate it for the duration of the longest time interval (12h). The availability of cells also precluded us from purifying LC, namely in the biochemical analysis attempted in section 5.4.3.

Most of our quantitative analysis employed a novel flow cytometric method using

\footnotetext{
1 LC make up 1-3\% of the total EC suspension but, because of the large excess of free particles in phagocytic assays, EC themselves only make up 5-10\% of the number of events crossing the laser beam. Consequently, in order to analyse the LC population, we effectively gate on $0.05-0.3 \%$ of the total number of events crossing the beam at a rate of $4000 / \mathrm{s}$.
} 
double colour fluorescence to identify LC and their association with particles or endocytic tracers. Like some other flow cytometry assays of phagocytosis [222], the assay employed here does not distinguish between attached and ingested particles. Two types of approaches have been devised to make this distinction possible. Sveum et al [265] used Texas Red and antibodies against Lucifer Yellow (LY) to detect LY-conjugated bacteria attached to phagocytes by two-colour flow cytometry; bacteria that were truly internalised were not accessible to the antibody and did not stain red. A similar two-colour flow cytometry approach has been used by others [266]. However, that approach, in our system, would have required triple colour flow-cytometry, a daunting prospect for anyone familiar with the technique. An alternative approach is to quench the fluorescence of external particles with trypan blue [267] or crystal violet and we used the former agent with success in experiments with $\mathrm{M} \varnothing$ (e.g. table 5.5). This approach, once again, could not be extended to LC because trypan blue also quenched the signal of the second fluorochrome used to identify the cells; use of the dye before staining the cells offered no advantage because quenching was rapidly reversible (fig. 2.5). However, we believe that the data obtained with our assay primarily reflect uptake rather than binding, at $37^{\circ} \mathrm{C}$, for the reasons outlined in section 2.5.2, although it is possible to detect a small amount of binding that can occur at $4^{\circ} \mathrm{C}$ (table 2.3 and fig. 4.4).

\section{3 - Phagocytosis of antigens by dendritic cells}

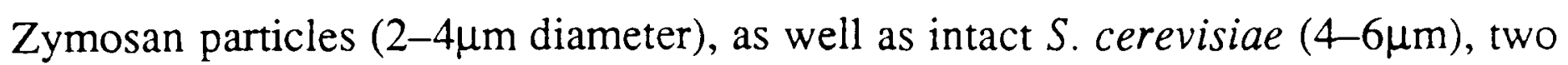
sorts of bacteria and $0.5-3.5 \mu \mathrm{m}$ latex microspheres, were phagocytosed by fresh but, largely, not by cultured LC (table 4.5). Both changes in the ultrastructure of the zymosan particles at the EM level (fig. 3.2e) and FACS analysis (table 4.2 and fig. 4.3) suggested that some degradation of internalised particles occurred in LC. Other workers have monitored the appearance of trichloroacetic acid-soluble radioactivity in the medium of MØ pulsed with 125 I-zymosan to measure particle degradation [223] but such an approach was not feasible in our system because we have observed that keratinocytes can also 
phagocytose zymosan (e.g. fig. 4.2a,b). Presumably, degradation of particulate antigens by LC is important for processing of internalised particles before presentation. In this respect, it is noteworthy that two very recent reports on infection of murine epidermal LC by Leishmania major concluded that up to $20 \%$ of these cells from BALB/c mice can be successfully infected in vitro and present parasite antigens to sensitised $\mathrm{T}$ cells and $\mathrm{T}$ cell clones $[231,251]$.

The uptake of zymosan was three to four times greater in C57Bl/6 LC compared to BALB/c LC (see section 3.4) and differential kinetics of zymosan uptake during cell maturation appeared to be responsible for this difference since C57Bl/6 LC showed an early rise in uptake, peaking at 6-8h, which was not found with BALB/c LC (fig. 4.4a; e.g. compare the sum of indices at times 0 and $6 \mathrm{~h}$ for the two strains). The rapid down regulation of zymosan uptake by fresh LC may also explain why uptake was very slow when measured continuously (fig. 3.6c). Subsequent investigation suggested that strain differences in zymosan phagocytosis might be due to differential strain-dependent expression of the receptors mediating zymosan uptake (see below). The increased phagocytic activity of $\mathrm{C} 57 \mathrm{Bl} / 6 \mathrm{LC}$ from time 0 to 6 h could conceivably be an artefact due to recovery from the trypsin used for isolation of the cells which could act preferentially on the receptors primarily responsible for zymosan uptake in this strain of LC, in keeping with the observation that $\mathrm{C} 57 \mathrm{Bl} / 6$ appeared to express higher levels of receptors for mannose and $\beta$-glucan which are trypsin-sensitive in $M \varnothing[236,255]$. To address this hypothesis experimentally we trypsinised $6 \mathrm{~h}$-cultured $\mathrm{C} 57 \mathrm{Bl} / 6 \mathrm{LC}$ to attempt to abrogate the peak in phagocytic activity but the experiment yielded no conclusive results and was not repeated (not shown).

Phagocytosis of the Gram-positive bacteria S. aureus (cocci; $0.7-1.2 \mu \mathrm{m}$ diameter) and $C$. parvum (bacilli; $2-6 \mu \mathrm{m}$ length, $0.5-1 \mu \mathrm{m}$ diameter) by LC was also observed (chapter 3). The two bacteria were taken up to a different extent and only about $50 \% \mathrm{LC}$ phagocytosed unopsonised $C$. parvum at saturation (fig. 3.8) suggesting there is heterogeneity in the phagocytic capacity even of fresh LC, a fact also implied by the 
finding that only 50-70\% BALB/c LC take up zymosan (section 3.4). In contrast, virtually $100 \%$ of $\mathrm{M} \emptyset$ phagocytosed S. aureus, C.parvum or zymosan when the particles were used at saturation (data not shown and table 5.2). It is possible that the heterogeneity observed with LC arises in part due to susceptibility of particular phagocytic receptors to trypsin or, alternatively, it represents sub-populations of LC with distinct phagocytic properties. Apart from the pathogenicity of these or related bacteria, S. aureus is of immunological interest because of the production of enterotoxins that can function as superantigens, and which are efficiently presented by DC [178].

In contrast to $\mathrm{M} \varnothing, \mathrm{LC}$ did not phagocytose aldehyde-fixed SRBC, IgG- or C'coated SRBC, and did not take up colloidal carbon (section 3.2). These results, once again, raise the possibility that trypsin-sensitive phagocytic receptors may be lost during cell preparation. This possibility can be excluded for uptake via Fc receptors and CR3. Murine LC in EC suspensions prepared exactly as here stain with $2.4 \mathrm{G} 2$ and M1/70, albeit weakly [120]. Witmer-Pack et al [136] deliberately trypsinised isolated murine LC cultured for 1 day and found no effect on expression of a variety of antigens, including CR3. In addition, human LC isolated by trypsinisation from epidermis form rosettes with $C^{\prime}-$ and IgGcoated erythrocytes [268, 269] and have been shown to express the trypsin-resistant Fc $\gamma$ RII $[220,269]$. Our finding that phagocytosis of zymosan by fresh LC is efficiently inhibited by mannan and $\beta$-glucan also suggests that trypsinisation used in preparation of the cells has a relatively mild effect since MR and $\beta$-glucan receptor are trypsin-sensitive (although we do not exclude some detrimental effect of the protease, as noted above). It is worth noting that, although high concentrations of trypsin $(0.625 \%)$ are routinely used for dissociation of epidermal cells, LC are probably exposed to significantly lower concentrations since they are protected by the dermis and stratum corneum during incubation of the ear-halves with the protease. We confirmed the presence of intact Fc and complement receptors in freshly-isolated LC (data not shown) but we did not find LC rosetted with IgG- or C'- opsonised SRBC in our experiments and this was not due to loss of rosetted cells during gradient centrifugation (section 3.2). Rather, since Fc receptors are 
lost very rapidly in culture [120], it is possible that initial rosettes of LC with IgG-coated SRBC were lost during the course of the assay (12-16h), although such a mechanism cannot explain why rosettes were not seen with CR3 which persists at low levels throughout culture [136]. The failure to observe phagocytosis of IgG-coated erythrocytes is consistent with the report that rat veiled cells do not take up these particles [89] but is in contrast with one report documenting their uptake by murine LC [186]. The unconventional procedure used to isolate the cells in the latter report makes it difficult to draw comparisons with the present work. It is possible that different LC sub-populations, preferentially selected by different isolation methods, express different forms of Fc $\gamma \mathrm{R}$ some of which are not capable of mediating phagocytosis, such as those found in B cells [119]. The fact that LC did not endocytose colloidal carbon is consistent with reports that uptake of this marker by rabbit VC cannot be detected in vitro $[87,88]$.

Phagocytosis of all particles decreased rapidly upon culture although LC remained viable and showed evidence of maturation (chapter 4). In theory, the apparent down regulation of uptake of particles and ManBSA could be due to a marked increased rate of degradation by cultured versus fresh LC. This is unlikely because cultured LC do not process intact antigens [130] and have lost acidic vesicles from the endocytic pathway where degradation is thought to take place [133], suggesting that, if anything, down regulation of uptake of FITC-zymosan and FITC-bacteria was underestimated due to more active degradation by fresh LC (latex particles, on the other hand, are non-degradable). In addition, down regulation of uptake of FITC-conjugates, as assessed by flow cytometry, may have been further underestimated because the fluorescence of fluorescein is partly quenched at low $\mathrm{pH}[270]$. Thus, the mean fluorescence of particles delivered to acidic phagosomes in fresh LC might be lower than that of the same number of particles ingested by cultured LC devoid of acidic compartments.

In the course of experiments with soluble tracers we also noted that the uptake of FITC-BSA (fig. 5.10b) and Lucifer Yellow (not shown) decreased during LC maturation after an initial rise when assayed with 90 min pulses at various times during culture 
suggesting that pinocytic activity was down regulated. In contrast, Puré and colleagues [35] have reported that the uptake of rhodamine-ovalbumin during a 4 h pulse was the same in cultured versus fresh LC and we have confirmed their observations using long (24h) incubations with FITC-BSA (fig. 5.8b). The discrepancy between the results obtained with short pulses and those obtained with long incubations may reflect the fact that the overall size of the late endocytic compartment is the same in cultured and fresh LC but the rate of uptake is decreased in mature LC. Re-routing of pinocytic tracer from early to late endosomes or vice-versa could also affect the rate of FITC-BSA accumulation as has been shown for Lucifer Yellow in M $\varnothing$ [258]. We have not tested these hypotheses but it seems likely that all the antigen uptake functions of LC (rate of endocytosis and phagocytosis) are down regulated as part of the maturation process. It is tempting to speculate that the decrease seen in phagocytic and endocytic activity during LC maturation could contribute to the loss of (native) antigen presentation by these cells.

Uptake of latex beads and zymosan by freshly isolated splenic DC was observed but this decreased to a low level after culture of the cells overnight (section 4.5). These results are broadly consistent with early reports that splenic DC do not phagocytose latex and other particles, in that populations of DC cultured overnight were studied [55], and that rat $\mathrm{VC}$ are also unable to take up latex particles [89]. One report that $\mathrm{VC}$ from sheep can take up latex [94] may be due to the maturational heterogeneity of these cells in afferent lymph and is consistent with our observations that some migratory LC are phagocytic (table 4.1. and data not shown). These observations indicate that, at least some, lymphoid and circulating DC can be phagocytic. Therefore, we believe it is likely that freshlyisolated lymphoid DC are heterogeneous and that our data reflect maturation of the less mature cells in culture with concomitant loss of phagocytic activity. Such a maturational process has recently been described by others (see section 1.2.3). Similar results to these were obtained for uptake of FITC $-S$. aureus and FITC $-C$. parvum by splenic DC (not shown) and it is noteworthy that the cell walls of corynebacteria are chemically and serologically related to those of mycobacteria [271]. DC are able to present whole 
Mycobacterium tuberculosis to T cells $[154,179]$ and, in the light of our data, presentation could be due to phagocytosis of this organism by a small number of DC, rather than because of extracellular processing as previously suggested [154]. Similarly, our results would seem to emphasise that presentation of $C$. parvum by splenic DC does not require the participation of $\mathrm{M} \varnothing$ for phagocytosis and processing, contrary to what has been proposed [180]. The relevance of these observations for induction of immune responses against blood-borne pathogens awaits investigation.

\section{4 - Receptors mediating phagocytosis by Langerhans cells}

Fresh LC express at least three different Fc receptors, namely Fc $\gamma \mathrm{R}$ (Fc $\gamma \mathrm{RII}$, CDw32, in humans), FceRI and FceRII (CD23), together with complement receptors (type 3; $\mathrm{CD} 11 \mathrm{~b} / \mathrm{CD} 18)$. At least Fc $\gamma \mathrm{R}$ is down regulated whereas CR3 is retained and, in mouse, CD11c/CD18 (complement receptor type 4; DC-specific epitope, N418) is up regulated during maturation of LC in culture. The uptake of soluble immune complexes by LC has been inferred from previous reports in which, for example, peroxidase injected subcutaneously into sensitised sheep was detected in "frilly" cells in draining lymph [93]. We have not found evidence for phagocytosis of ligands for FcyR or CR3 (see above) but uptake of $C$. parvum was markedly increased by pre-incubation in fresh mouse serum. This observation could suggest that phagocytosis via complement and/or Fc receptors may require co-ligation of other receptors. For example, in the case of monocytes and $M \varnothing$, uptake of particles via CR3 only occurs in the presence of fibronectin, laminin or serum amyloid $\mathrm{P}$, unless the cells are activated, and similar uptake by neutrophils requires both fibronectin and additional exposure to chemotactic peptides (see section 1.3.2). Therefore, Fc and/or complement receptors on LC could conceivably mediate phagocytosis only when the cells interact with components of the extracellular matrix (i.e., in situ but not in vitro) and/or inflammatory mediators, or they could synergise with other receptors such as mannose receptor (see below). This would explain why erythrocytes have been observed within LC migrating from skin explants (and in VC in vivo) but cannot be phagocytosed in 
vitro (fig. 3.3) and why LC (and IDC) are able to phagocytose apoptotic cells in situ (see section 1.2.5).

We investigated the receptors mediating phagocytosis of zymosan by Langerhans cells. Inhibition studies demonstrated that zymosan uptake could be specifically inhibited by mannan and $\beta$-glucan but, in contrast to $M \varnothing$ [223], not by a monoclonal antibody (M1/70) against CR3 (table 5.4). Our inhibition assays routinely lasted $12-16 \mathrm{~h}$, and it is possible that inhibition with anti-CR3 was not detectable after such a long incubation, in line with the observation that the contribution of CR3 to zymosan phagocytosis is shortlived in $M \varnothing$ [223]. However, Blank et al, in a report soon to be published [251], have found that infection of LC by L. major can be completely inhibited by M1/70 $(200 \mu \mathrm{g} / \mathrm{ml})$ during a $24 \mathrm{~h}$ assay, suggesting that, if M1/70 inhibited zymosan uptake by LC, the effect would not be masked by the length of the assay. Surprisingly, infection of BALB/c LC by L. major does not appear to be inhibitable by mannan [251] although mannose receptors, together with CR3, mediate infection of macrophages by L. donovani [257, 272]. Intriguingly, we were never able to inhibit zymosan uptake by resident $M \varnothing$ with $M 1 / 70$, even using a short, $10 \mathrm{~min}$ assay (table 5.5). Our observations suggest that CR3 does not play a major role in the phagocytosis of zymosan in LC although we cannot exclude the possibility that it co-operates with glycan receptors in the initial stages of phagocytosis, particularly in the light of the report by Graham and colleagues [216] suggesting that a immobile subset of CR3 functions in mobilising the cytoskeleton for phagocytosis and is involved in phagocytosis mediated by other receptors (see section 1.3). There is also evidence that co-operation between CR3 and putative $\beta$-glucan receptors may mediate uptake of opsonised zymosan by human neutrophils [273].

Mannose and $\beta$-glucan receptors have not previously been demonstrated in DC and rat VC do not bind ${ }^{125}$ I-ManBSA specifically [89]. Our results support the involvement of $\beta$-glucan-inhibitable and mannose-type receptors in the phagocytosis of zymosan by LC (section 5.2.1) but it is important to reiterate that our data do not address the relationship between these recognition units and those found in professional phagocytes. Furthermore. 
our data do not exclude the possibility that a single receptor is responsible for binding of both ligands. Thus, although, for convenience, we talk about $\beta$-glucan and mannose receptors, we acknowledge the possibility that they represent a novel receptor(s), different from those described in mononuclear phagocytes. However, it is tempting to speculate that they are the same receptors of on the basis of other similarities between LC and M $\varnothing$, such as expression of $\mathrm{F} 4 / 80,2.4 \mathrm{G} 2$ and $\mathrm{M} 1 / 70$ antigens (table 1.1). Based on this working hypothesis, we shall discuss our findings in the context of published reports on $\beta$-glucan and mannose receptors.

Highly purified soluble yeast glucan was a more potent inhibitor of zymosan phagocytosis on a weight basis than pure mannan for LC (figs. 5.1-5.4) and for J774E macrophages (table 5.6). Similar observations have been made for thio-M $\varnothing$ [237] and bone marrow-derived $\mathrm{M} \varnothing$ [236] which have both mannose and $\beta$-glucan receptors although inhibition with mannan was attributed to contaminating $\beta$-glucan in those experiments [237]. Our mannan did not contain detectable glucose residues and, therefore, our experiments support the notion that inhibition with mannan was a function of, previously undescribed, mannose receptors in LC. Interestingly, on a weight basis, zymosan particles contain primarily glucan (50-60\%) as opposed to mannan (16-23\%) [228] which may explain why soluble $\beta$-glucan exerts a more profound inhibitory effect than mannan, even if equivalent amounts of the two receptors are expressed. It is possible that mannose and $\beta$-glucan receptors synergise in the uptake of zymosan by LC since greater than $50 \%$ inhibition of uptake was observed when each glycan was used in isolation (figs. 5.3-5.5). We have not tried mannan and glucan in combination to establish whether complete inhibition of zymosan uptake can be achieved. The differential inhibition of uptake seen in $\mathrm{C} 57 \mathrm{Bl} / 6$ versus $\mathrm{BALB} / \mathrm{C}$ LC suggested that receptors for these glycans played a more important role in the uptake of zymosan in the former strain LC (section 5.2.1). Inhibition by both glycans was lost during culture (fig. 5.5) implying that the availability of the receptors involved was being down regulated and this was confirmed in the case of mannose receptors by studies on the uptake of ManBSA (see below). However, 
for the reasons noted in section 5.3.1, these experiments do not allow us to state that physical down regulation of the receptors occurred during LC maturation.

A role for MR in host defence has been proposed, on the basis that bacteria are not capable of processing high-mannose type chains and, like yeasts, express a variety of mannans and glucans on their cell walls [241]. $\beta$-glucan receptors could have a similar role and it is noteworthy that uptake of particles other than yeasts, for example, Mycobacterium avium, can be partially inhibited with mannan and/or $\beta$-glucan [274]. It, therefore, seems appropriate that LC should express mannan and $\beta$-glucan receptors in order to acquire micro-organisms for presentation. Indeed, the possibility that fresh but not cultured LC express MR was entertained by Puré and colleagues who had observed uptake of horseradish peroxidase, a high-mannose type protein, in fresh but not cultured LC [35]. ${ }^{2}$ We have examined the presence and fate of mannose-type receptors in LC during maturation in vitro using ManBSA, a neoglycoprotein introduced by Stahl and colleagues [275] which has been widely used as a probe for M $\varnothing$ MR. Fresh C57Bl/6 LC endocytosed FITC-ManBSA specifically (fig. 5.7) and specific uptake was down regulated in culture (figs. 5.8, 5.9 and 5.10a) with kinetics similar to those previously observed for zymosan uptake (fig. 4.4). These results suggest functional expression of mannose-type receptors is down regulated during maturation as noted above for inhibition studies with mannan. ManBSA is rapidly degraded in early endosomes [31] and the signal detected by FACS at saturation is likely to represent a balance between accumulation of tracer and intracellular degradation [255]. As such, down regulation of uptake may have been underestimated since fresh LC are thought to degrade exogenous antigens more actively than cultured cells. In addition, down regulation may also have been underestimated because of quenching of fluorescein at low $\mathrm{pH}$, as noted before. Direct measurement of the levels of receptor expressed on the plasma membrane of LC was not possible because it was not feasible to purify sufficient numbers of these cells for receptor-ligand studies, e.g. using 125I-ManBSA, and because we were unable to enhance the signal for FACS analysis of

2 Unpublished observation mentioned in discussion. 
cells labelled with FITC-ManBSA at $4^{\circ} \mathrm{C}$ (fig. 5.11). The failure to detect a signal in binding assays may be due to the fact that, as in MØ, MR in LC is mostly intracellular.

It is important to stress that ManBSA and mannan are not specific for the macrophage mannose receptor and, indeed, studies on MR published to date do not preclude the possibility that the activity found may be due to more than one receptor or isoforms thereof. In line with this hypothesis is the fact that the molecular weight of mature MR has been variously estimated at $162-175 \mathrm{kD}$ [260-264], even by the same workers using the same source of material ([261, 263]; S. Pontow, personal communication). Mannose receptors other than the $162-175 \mathrm{kD}$ MR have also been described, namely a $60 \mathrm{kD}$ receptor in a human promyelocytic cell line which binds FITC-ManBSA [276]. To determine whether mannose receptor activity in LC was due to expression of the macrophage mannose receptor we made use of a novel polyclonal antibody against murine MR in collaboration with Prof. P. D. Stahl, Washington University. This reagent was used successfully in biochemical studies of MØ MR (section 5.4.1) but proved to be of little use in immunofluorescence studies and did not inhibit uptake of MR ligands (section 5.4.2) Surprisingly, BALB/c thio-M $\varnothing$ seemed to express less mannose receptor than either $\mathrm{C} 57 \mathrm{Bl} / 6$ thio-M $\varnothing$ or J774E cells (figs. 5.10 and 5.11). It is interesting to speculate that $\mathrm{BALB} / \mathrm{c} \mathrm{M} \varnothing$ and LC express less MR than $\mathrm{C} 57 \mathrm{~B} 1 / 6$ cells but the functional manifestation of this difference is only seen in LC which are poorly phagocytic cells. In MØ, small variations in expression of mannose receptors are likely to be compensated by the high rate of zymosan phagocytosis and the fact that multiple receptors are capable of mediating phagocytosis of these particles (table 5.1). We have not yet been able to extend the biochemical characterisation of MR to Langerhans cells due to the difficulty in purifying sufficient numbers of LC for protein analysis (section 5.4.3). It may be prove more productive to attempt to quantify MR message by polymerase chain reaction as was done recently for $\mathrm{M} \varnothing[277]$.

The possibility that down regulation of specific phagocytic receptors during maturation of LC contributes directly to their loss (or very marked reduction) of phagocytic 
activity is suggested by the fascinating observations that the capacity to internalise ligands is conferred upon cells by particular receptors. For example, transfection of human macrophage mannose receptor cDNA into non-phagocytic COS-1 cells allows them to phagocytose yeasts [202], and transfection of FcyRII into fibroblasts allows them to phagocytose $T$. gondii [207]. Similarly, transfection of the macrophage (B2) form of FcyRII into B cells (which normally express a similar receptor containing an insertion in the cytoplasmic domain and which mediates binding and signal transduction but not internalisation) results in endocytosis of immune complexes [278].

\section{5 - The relevance of phagocytosis by Langerhans cells}

It is clear from the data presented in this thesis that particles are phagocytosed by Langerhans cells more slowly and to a lesser extent than by macrophages (chapter 3 ). Furthermore, unlike the latter cell type, LC show an exquisite selectivity in their phagocytic capacity in vitro and are unable to take up particles via Fc $\gamma$ receptors or bind them via complement receptors. This would appear to reflect the primary function of LC, which is to internalise and process antigens, for presentation to resting $\mathrm{T}$ cells and initiation of an immune response, as opposed to scavenging and clearance by $\mathrm{M} \varnothing$ (although M $\varnothing$ can present antigens to $\mathrm{T}$ cells once sensitised by $\mathrm{DC}$ ). We believe that freshly-isolated LC are a model for immature DC and our results support the view that the function of immature DC resident in non-lymphoid and in lymphoid tissues is to acquire antigens, particulate and soluble, for presentation. For example, studies from our laboratory have shown that mouse heart and kidney dendritic cells are immature in situ and, based on the findings in this study, these DC were shown to be capable of phagocytosing small amounts of zymosan in vitro, presumably through the same receptors expressed by LC (J. Roake, A.S. Rao et al, unpublished observations). Furthermore, as for LC, kidney DC from $\mathrm{C} 57 \mathrm{Bl} / 6$ mice were more phagocytic for zymosan than those from BALB/c (A. S. Rao, personal communication). Our findings that LC are poorly phagocytic compared to "professional" phagocytes emphasise that, in cells where the processing machinery is well 
developed (i.e. high rate of MHC class II and invariant chain synthesis and abundance of acidic organelles), as in immature DC such as fresh LC, only very small amounts of antigen will suffice to stimulate antigen-specific $T$ cells. In contrast, the targetting of antigens to the surface immunoglobulin ( $\mathrm{SIg}$ ) in B cells, which leads to strongly enhanced processing by these cells, is due to the fact that via sIg B cells take up much more antigen $[27,29]$. The uniqueness and specialisation of immature DC in that regard may be that they do not have to rely on this property since other parts of the antigen processing mechanism are so well developed. It is also not surprising that fresh LC do not phagocytose antigens via Fc $\gamma$ or complement receptors. It would not make sense for a cell that initiates immune responses to have to rely on specific antibody for particle uptake (although natural antibodies could play a role). Instead, LC express broad-spectrum phagocytic receptors, such as MR and $\beta$-glucan receptor, which are capable of recognising a wide range of micro-organisms through common carbohydrate determinants in their cell walls. In accordance with the model established for DC function (see section 1.2), we believe that LC that have phagocytosed antigen function to transport it to lymphoid tissues where presentation occurs. We performed some crude experiments where FITC-zymosan was painted onto lightly-abraded mouse ears and the draining lymph nodes and ears were excised $48 \mathrm{~h}$ later. We were unable to detect FITC-zymosan-labelled cells in cell suspensions of draining LN but we observed $\mathrm{Ia}^{+}$cells that had phagocytosed particles amongst the cells that migrated out of ear skin after organ culture, suggesting that the cells had phagocytosed in situ before entering the skin lymphatics (unpublished observations). In this respect, it is noteworthy that $L$. major-pulsed LC appear to carry parasites to the draining lymph nodes following intradermal administration ( $H$. Moll, personal communication).

Our findings do not exclude an additional role for LC in the control of local immune responses in the skin and it is possible that $\mathrm{LC}$ perform $\mathrm{M} \varnothing$ effector functions such as production of oxygen radicals and nitric oxide. We carried out preliminary experiments looking for an oxidative burst during phagocytosis of zymosan by LC but did 
not obtain conclusive results and did not pursue the experiments further. Interestingly, in this context, replication of Leishmania internalised by LC appears to be inhibited but the mechanisms involved are not known (H. Moll, personal communication). However, Kaplan et al [279] have not found any effect of GM-CSF-induced LC accumulation at leprous lesions on the number of bacilli that were present. Effector functions of LC and whether certain pathogens have evolved mechanisms to prevent uptake, or processing and presentation by cells of this lineage remain to be investigated.

Phagocytosis by DC offers new perspectives into the role played by these cells in disease. For example, the composition of cell walls is remarkably similar between different yeasts [250] and the finding that fresh LC phagocytose zymosan offers an explanation as to how "non-phagocytic" DC could play a role in the initiation of cell-mediated immunity to Candida. Whether strain differences in resistance or susceptibility to L. major (i.e., induction of $\mathrm{T}_{\mathrm{H}} 1$ or $\mathrm{T}_{\mathrm{H}} 2$ responses), and other parasites, between inbred mice are related to differences in the phagocytic capacity of LC remains to be investigated. It is, however, a fascinating possibility given the strain differences observed for phagocytosis of zymosan in this thesis.

The definition of phagocytic and endocytic receptors in LC may also be of clinical importance. For example, in relation to vaccination, a recent report highlighting the adjuvanticity of mannan when used as a carrier for immunogenic peptides may have been due to targetting to immature dendritic cells [280]. These receptors could also be targets for cytotoxic agents to deplete passenger leukocytes from organs before transplantation, as was shown with in vivo depletion of Kupffer cells from rat and mouse liver using ricin $[281,282]$, a toxin internalised in part via the mannose receptor [283]. Finally, the study of these receptors would be expected to lead to new insights into pathways of antigen processing by immature dendritic cells. 


\section{REFERENCES}

1. Monaco, J.J. 1992. A molecular model of MHC class-I-restricted antigen processing. Immunol. Today 13: 173-179.

2. Neefjes, J.J. and H.L. Ploegh. 1992. Intracellular transport of MHC class II molecules. Immunol. Today 13: 179-184.

3. Austyn, J.M. 1989. Antigen-presenting cells. In Focus Series, Oxford: IRL Press.

4. Brodsky, F.M. and L.E. Guagliardi. 1991. The cell biology of antigen processing and presentation. Annu. Rev. Immunol. 9: 707-744.

5. Braciale, T.J. and V.L. Braciale. 1991. Antigen presentation: structural themes and functional variations. Immunol. Today 12: 124- 129.

6. Murray, N. and A. McMichael. 1992. Antigen presentation in virus infection. Curr. Opin. Immunol. 4: 401-407.

7. Bjorkman, P.J., M.A. Saper, B. Samraoui, W.S. Bennett, J.L. Strominger, and D.C. Wiley. 1987. Structure of human class I histocompatibility antigen, HLA-A2. Nature 329: 506-512.

8. Yewdell, J.W. and J.R. Bennik. 1989. Brefeldin a specifically inhibits presentation of protein antigens to cytotoxic T lymphocytes. Science 244: 1072- 1075.

9. Nuchtern, J.G., J.S. Bonifacino, W.E. Biddison, and R.D. Klausner. 1989. Brefeldin A implicates egress from endoplasmic reticulum in class I restricted antigen presentation. Nature 339: 223-226.

10. Townsend, A., C. Öhlén, J. Bastin, H.-G. Llunggren, L. Foster, and K. Kärre. 1989. Association of class I major histocompatibility heavy and light chains induced by viral peptides. Nature 340: 443-448.

11. Powis, S.J., A.R.M. Townsend, E.V. Deverson, J. Bastin, G.W. Butcher, and J.C. Howard. 1991. Restoration of antigen presentation to the mutant cell line RMA-S by an MHC-linked transporter. Nature 354: 528-531.

12. Powis, S.J., E.V. Deverson, W.J. Coadwell, A. Ciruela, N.S. Huskisson, H. Smith, G.W. Butcher, and J.C. Howard. 1992. Effect of polymorphism of an MHC-linked transporter on the peptides assembled in a class I molecule. Nature 357: 211-215.

13. Lippincott-Schwartz, J., J.S. Bonifacino, L.C. Yuan, and R.D. Klausner. 1988. Degradation from the endoplasmic reticulum: disposing of newly synthesised proteins. Cell 54: 209-220. 
14. Neefjes, J.J., M.L.H. De Bruijn, C.J.P. Boog, J.D. Nieland, J. Boes, C.J.M. Melief. and H.L. Ploegh. 1990. N-linked glycan modification on antigen-presenting cells restores an allospecific cytotoxic T cell response. J. Exp. Med. 171: 583-588.

15. Rock, K.L., L. Rothstein, and B. Benacerraf. 1992. Analysis of the association of peptides of optimal length to class I molecules on the surface of cells. Proc. Natl. Acad. Sci. U.S.A. 89: 8918-8922.

16. Stockinger, B., U. Pessara, R.H. Lin, J. Habicht, M. Grez, and N. Koch. 1989. A role of Ia-associated invariant chains in antigen processing and presentation. Cell 56: $683-689$.

17. Peterson, M. and J. Miller. 1990. Invariant chain influences the immunological recognition of MHC class II molecules. Nature 345: 172- 174.

18. Peterson, M. and J. Miller. 1992. Antigen presentation enhanced by the alternatively spliced invariant chain gene product. Nature 357: 596-598.

19. Lotteau, V., L. Teyton, A. Peleraux, T. Nilsson, L. Karlsson, S.L. Schmid, V. Quaranta, and P.A. Peterson. 1990. Intracellular transport of class II MHC molecules directed by invariant chain. Nature 348: 600-605.

20. Lamb, C.A., J.W. Yewdell, J.R. Bennink, and P. Cresswell. 1991. Invariant chain targets HLA class II molecules to acidic endosomes containing internalized influenza virus. Proc. Natl. Acad. Sci. U.S.A. 88: 5998-6002.

21. Bakke, O. and B. Dobberstein. 1990. MHC class II-associated invariant chain contains a sorting signal for endosomal compartments. Cell 63: 707-716.

22. Roche, P.A. and P. Cresswell. 1990. Invariant chain association with HLA-DR molecules inhibits immunogenic peptide binding. Nature 345: 615-618.

23. Guagliardi, L.E., B. Koppelman, J.S. Blum, M.S. Marks, P. Cresswell, and F.M. Brodsky. 1990. Co-localization of molecules involved in antigen processing and presentation in an early endocytic compartment. Nature 343: 133- 139.

24. Neefjes, J.J., V. Stolloz, P.J. Peters, H.J. Geuze, and H.L. Ploegh. 1990. The biosynthetic pathway of $\mathrm{MHC}$ class II but not class I molecules intersects the endocytic route. Cell 61: 171- 183 .

25. Alberts, B., D. Bray, J. Lewis, M. Raff, K. Roberts, and J.D. Watson. 1983. Molecular Biology of the Cell. New York: Garland Publishing Inc.

26. Rabinowitz, S., H. Horstmann, S. Gordon, and G. Griffiths. 1992. Immunocytochemical characterization of the endocytic and phagolysosomal compartments in peritoneal macrophages. J. Cell Biol. 116: 95-112.

27. Lanzavecchia, A. 1990. Receptor-mediated antigen uptake and its effect on antigen presentation to class II-restricted T lymphocytes. Annu. Rev. Immunol. 8: 773-793.

28. Stein, M. and S. Keshav. 1992. The versatility of macrophages. Clin. Exp. Allergy 


\section{2: 19-27.}

29. Stockinger, B. 1992. Capacity of antigen uptake by B cells, fibroblasts or macrophages determines efficiency of presentation of a soluble self antigen (C5) to T lymphocytes. Eur. J. Immunol. 22: 1271-1278.

30. Steinman, R.M., I.S. Mellman, W.A. Muller, and Z.A. Cohn. 1983. Endocytosis and the recycling of plasma membrane. J. Cell Biol. 96: 1-27.

31. Diment, S. and P. Stahl. 1985. Macrophage endosomes contain proteases which degrade endocytosed protein ligands. J. Biol. Chem. 260: 15311-15317.

32. Blum, J.S., M.L. Fiani, and P.D. Stahl. 1991. Proteolytic cleavage of ricin A chain in endosomal vesicles: evidence for the action of endosomal proteases at both neutral and acidic pH. J. Biol. Chem. 266: 22091-22095.

33. Davidson, H.W., M.A. West, and C. Watts. 1990. Endocytosis, intracellular trafficking, and processing of membrane IgG and monovalent antigen/membrane IgG complexes in B lymphocytes. J. Immunol. 144: 4101-4109.

34. Peters, P.J., J.J. Neefjes, V. Oorschot, H.L. Ploegh, and H.J. Geuze. 1991. Segregation of MHC class II molecules from MHC class I molecules in the Golgi complex for transport to lysosomal compartments. Nature 349: 669-676.

35. Puré, E., K. Inaba, M.T. Crowley, L. Tardelli, P.M. Witmer, G. Ruberti, G. Fathman, and R.M. Steinman. 1990. Antigen processing by epidermal Langerhans cells correlates with the level of biosynthesis of major histocompatibility complex class II molecules and expression of invariant chain. J. Exp. Med. 172: 1459-69.

36. Harding, C., R.W. Roof, and E.R. Unanue. 1989. Turnover of Ia-peptide complexes is facilitated in viable antigen-presenting cells: biosynthetic turnover of Ia vs. peptide exchange. Proc. Natl. Acad. Sci. U.S.A. 86: 4230-4234.

37. Harding, C.V. and E.R. Unanue. 1989. Antigen processing and intracellular Ia. Possible roles of endocytosis and protein synthesis in Ia function. J. Immunol. 142: $12-19$.

38. Adorini, L., S.J. Ullrich, E. Appella, and S. Fuchs. 1990. Inhibition by brefeldin A of presentation of exogenous protein antigens to MHC class II-restricted T cells. Nature 346: 63-66.

39. Davidson, H.W., P.A. Reid, A. Lanzavecchia, and C. Watts. 1991. Processed antigen binds to newly synthesized MHC class II molecules in antigen-specific B lymphocytes. Cell 67: 105-116.

40. Adorini, L., E. Apella, G. Doria, F. Cardinaux, and Z.A. Nagy. 1989. Competition for antigen presentation in living cells involves exchange of peptides bound by class II MHC molecules. Nature 342: 800-803.

41. Steinman, R.M. 1991. The dendritic cell system and its role in immunogenicity. 
Annu. Rev. Immunol. 9: 271-296.

42. Inaba, K. and R.M. Steinman. 1984. Resting and sensitized T lymphocytes exhibit distinct stimulatory (antigen-presenting cell) requirements for growth and lymphokine release. J. Exp. Med. 160: 1717-1735.

43. Inaba, K., S. Koide, and R.M. Steinman. 1985. Properties of memory T lymphocytes isolated from the mixed lymphocyte reaction. Proc. Natl. Acad. Sci. U.S.A. 82: 7686-7690.

44. Inaba, K., J.P. Metlay, M.T. Crowley, and R.M. Steinman. 1990. Dendritic cells pulsed with protein antigens in vitro can prime antigen-specific, MHC-restricted $\mathrm{T}$ cells in situ. J. Exp. Med. 172: 631-40.

45. Crowley, M., K. Inaba, and R.M. Steinman. 1990. Dendritic cells are the principal cells in mouse spleen bearing immunogenic fragments of foreign proteins. J. Exp. Med. 172: 383-6.

46. Inaba, K., J.W. Young, and R.M. Steinman. 1987. Direct activation of CD8 ${ }^{+}$ cytotoxic T lymphocytes by dendritic cells. J. Exp. Med. 166: 182-94.

47. Boog, C.J.P., J. Boes, and C.J.M. Melief. 1988. Stimulation with dendritic cells decreases or obviates the $\mathrm{CD} 4^{+}$helper cell requirement in cytotoxic T lymphocyte responses. Eur. J. Immunol. 18: 219-223.

48. Young, J.W. and R.M. Steinman. 1990. Dendritic cells stimulate primary human cytolytic lymphocyte responses in the absence of $\mathrm{CD}^{+}{ }^{+}$helper cells. J. Exp. Med. 171: 1315-1332.

49. Kast, W.M., C.J.P. Boog, B.O. Roep, A.C. Voordouw, and C.J.M. Melief. 1988. Failure or success in the restoration of virus-specific cytotoxic $\mathrm{T}$ lymphocyte response defects by dendritic cells. J. Immunol. 140: 3186-3193.

50. Macatonia, S.V., P.M. Taylor, S.C. Knight, and B.A. Askonas. 1989. Primary stimulation by dendritic cells induces antiviral proliferative and cytotoxic $\mathrm{T}$ cell responses in vitro. J. Exp. Med. 169: 1255-1264.

51. Steinman, R.M. and Z.V. Cohn. 1973. Identification of a novel cell type in peripheral lymphoid organs of mice: I. Morphology, quantitation, tissue distribution. J. Exp. Med. 137: 1142-1162.

52. Fossum, S. 1989. The life history of dendritic leukocytes (DL). In Current Topics in Pathology. O.H. Ivessen, editor. Springer-Verlag: Berlin. 101-24.

53. Hart, D.N.J. and J.W. Fabre. 1981. Demonstration and characterization of Iapositive dendritic cells in the interstitial connective tissues of rat heart and other tissues, but not brain. J. Exp. Med. 153: 347-361.

54. Freudenthal, P.S. and R.M. Steinman. 1990. The distinct surface of human blood dendritic cells, as observed after an improved isolation method. Proc. Natl. Acad. 
Sci.U.S.A. 87: 7698-702.

55. Steinman, R.M. and Z. Cohn. 1974. Identification of a novel cell type in peripheral lymphoid organs of mice: II. Functional properties in vitro. J. Exp. Med. 139: 380397.

56. Knight, S.C., B.M. Balfour, J. O'Brien, L. Buttifant, T. Sumerska, and J. Clarke. 1982. Role of veiled cells in lymphocyte activation. Eur. J. Immunol. 12: 10571060.

57. Steinman, R.M., W.C. Van Voorhis, and D.M. Spalding. 1986. Dendritic cells. In Handbook of Experimental Immunology. 2. Cellular immunology. D.M. Weir, L.A. Herzenberg, C. Blackwell, and L.A. Herenberg, editors. Blackwell Scientific Publications: Oxford. 49.1-49.9.

58. Mayrhofer, G., C. Pugh, and A. Barclay. 1983. The distribution, ontogeny and origin in the rat of Ia-positive cells with dendritic morphology and of Ia antigen in epithelia with special reference to the intestine. Eur. J. Immunol. 13: 112-122.

59. Witmer, M.D. and R.M. Steinman. 1984. The anatomy of peripheral lymphoid organs with emphasis on accessory cells: light-microscopic immunocytochemical studies of mouse spleen, lymph node, and Peyer's patch. Am. J. Anat. 170: 465481.

60. Spalding, D.M., W. Koopman, J. Eldridge, J. McGhee, and R. Steinman. 1983. Accessory cells in murine Peyer's patch: I. Identification and enrichment of a functional dendritic cell. J. Exp. Med. 157: 1646-1659.

61. Pavli, P., C.E. Woodhams, W.F. Doe, and D.A. Hume. 1990. Isolation and characterization of antigen-presenting dendritic cells from the mouse intestinal lamina propria. Immunol. 70: 40-47.

62. Nussenzweig, M.C., R.M. Steinman, M.D. Witmer, and B. Gutchinov. 1982. A monoclonal antibody specific for mouse dendritic cells. Proc. Natl. Acad. Sci. USA 79: 161-165.

63. Crowley, M., K. Inaba, P.M. Witmer, and R.M. Steinman. 1989. The cell surface of mouse dendritic cells: FACS analyses of dendritic cells from different tissues including thymus. Cell. Immunol. 118: 108-25.

64. Kyewski, B., C. Fathman, and R. Rouse. 1986. Intrathymic presentation of circulating non-MHC antigens by medullary dendritic cells: an antigen-dependent microenvironment for T cell differentiation. J. Exp. Med. 163: 231-246.

65. Landry, D., M. Lafontaine, M. Cossette, H. Barthelemy, C. Chartrand, S. Montplaisir, and M. Pelletier. 1988. Human thymic dendritic cells. Characterization, isolation and functional assays. Immunol. 65: 135-42.

66. Oliver, P. and N. LeDouarin. 1984. Avian thymic accessory cells. J. Immunol. 132: 1748-1755. 
67. Hart, D.N. and J.L. McKenzie. 1988. Isolation and characterization of human tonsil dendritic cells. J. Exp. Med. 168: 157-70.

68. King, P. and D. Katz. 1989. Human tonsillar dendritic cell-induced T cell responses: analysis of molecular mechanisms using monoclonal antibodies. Eur. $J$. Immunol. 19: 581-587.

69. Steinman, R.M., J.C. Adams, and Z.A. Cohn. 1975. Identification of a novel cell type in peripheral lymphoid organs of mice: IV.Identification and distribution in mouse spleen. J. Exp. Med. 141: 804-820.

70. Kraal, G., M. Breel, M. Janse, and G. Bruin. 1986. Langerhans' cells, veiled cells, and interdigitating cells in the mouse recognized by a monoclonal antibody. J. Exp. Med. 163: 981-97.

71. Dijkstra, C.D. 1982. Characterization of nonlymphoid cells in rat spleen, with special reference to strongly Ia-positive branched cells in $\mathrm{T}$-cell areas. $J$. Reticuloendoth. Soc. 32: 167-178.

72. Barclay, A.N. and G. Mayrhofer. 1981. Bone marrow origin of Ia-positive cells in the medulla of rat thymus. J. Exp. Med. 153: 1666-1671.

73. Austyn, J.M., J.W. Kupiec-Weglinski, D.F. Hankins, and P.J. Morris. 1988. Migration patterns of dendritic cells in the mouse. Homing to $\mathrm{T}$ cell-dependent areas of spleen, and binding within marginal zone. J. Exp. Med. 167: 646-51.

74. Fossum, S. 1988. Lymph-borne dendritic leukocytes do not recirculate, but enter the lymph node paracortex to become interdigitating cells. Scand. J. Immunol. 27: 97-105.

75. Metlay, J.P., P.M. Witmer, R. Agger, M.T. Crowley, D. Lawless, and R.M. Steinman. 1990. The distinct leukocyte integrins of mouse spleen dendritic cells as identified with new hamster monoclonal antibodies. J. Exp. Med. 171: 1753-71.

76. Lowe, J., K.A. MacLennan, D.G. Powe, J.D. Pound, and J.B. Palmer. 1989. Microglial cells in human brain have phenotypic characteristics related to possible function as dendritic antigen presenting cells. J. Pathol. 159: 143-149.

77. Holt, P.G., M.A. Schon-Hegrad, and J. Oliver. 1988. MHC class II antigen-bearing dendritic cells in pulmonary tissues of the rat. Regulation of antigen presentation activity by endogenous macrophage populations. J. Exp. Med. 167: 262-274.

78. Pollard, A.M. and M.F. Lipscomb. 1990. Characterization of murine lung dendritic cells: similarities to Langerhans cells and thymic dendritic cells. J. Exp. Med. 172: 159-67.

79. Gong, J.L., K.M. McCarthy, J. Telford, T. Tamatani, M. Miyasaka, and E.E. Schneeberger. 1992. Intraepithelial airway dendritic cells: a distinct subset of pulmonary dendritic cells obtained by microdissection. J. Exp. Med. 175: 797-807. 
80. Austyn, J.M. and R.M. Steinman. 1988. The passenger leukocyte- a fresh look. Transplant. Rev. 2: 139-176.

81. Faustman, D.L., R.M. Steinman, H.M. Gebel, V. Hauptfeld, J.M. Davie, and P.E. Lacy. 1984. Prevention of rejection of murine islet allografts by pretreatment with anti-dendritic cell antibody. Proc. Natl. Acad. Sci. U.S.A. 81: 3864-3868.

82. Romani, N. and G. Schuler. 1992. The immunologic properties of epidermal Langerhans cells as a part of the dendritic cell system. Springer Semin. Immunopathol. 13: 265-279.

83. Van Voorhis, W.C., L.S. Hair, R.M. Steinman, and G. Kaplan. 1982. Human dendritic cells: enrichment and characterization from perpheral blood. J. Exp. Med. 155: 1172-1187.

84. Van Voorhis, W.C., J. Valinsky, E. Hoffman, J. Lubman, L.S. Hair, and R.M. Steinman. 1983. Relative efficacy of human monocytes and dendritic cells as accessory cells for T cell replication. J. Exp. Med. 158: 174-191.

85. Knight, S.C., J. Farrant, A. Bryant, A.J. Edwards, S. Burman, A. Lever, J. Clarke, and D.B. Webster. 1986. Non-adherent, low density cells from human peripheral blood contain dendritic cells and monocytes, both with veiled morphology. Immunol. 57: 595-603.

86. Inaba, K., R.M. Steinman, M. Witmer Pack, H. Aya, M. Inaba, T. Sudo, S. Wolpe, and G. Schuler. 1992. Identification of proliferating dendritic cell precursors in mouse blood. J. Exp. Med. 175: 1157-1167.

87. Kelly, R.H., B. Balfour, and J. Armstrong. 1976. Lymph-borne Langerhans cells [Abstract 114]. Am. J. Path. 82: 85a.

88. Kelly, R.H., B.M. Balfour, J.A. Armstrong, and S. Griffiths. 1978. Functional anatomy of lymph nodes. II. Peripheral lymph-borne mononuclear cells. Anat. Rec. 190: 5-22.

89. Pugh, C.W., G.G. MacPherson, and H.W. Steer. 1983. Characterization of nonlymphoid cells derived from rat peripheral lymph. J. Exp. Med. 157: 1758-79.

90. Rhodes, J.M. 1985. Isolation of large mononuclear Ia-positive veiled cells from the mouse thoracic duct. J. Immunol. Meth. 85: 383.

91. Rhodes, J.M., B.M. Balfour, J. Blom, and R. Agger. 1989. Comparison of antigen uptake by peritoneal macrophages and veiled cells from the thoracic duct using isotope-, FITC-, or gold-labelled antigen. Immunol. 68: 403-9.

92. Spry, C.J.F., A.J. Pflug, G. Janossy, and J.H. Humphrey. 1980. Large mononuclear (veiled) cells with "Ia-like" membrane antigens in human afferent lymph. Clin. Exp. Immunol. 39: 750-755.

93. Hall, J.G. and D. Robertson. 1984. Phagocytosis, in vivo, of immune complexes by 
dendritic cells in the lymph of sheep. Int. Arch. Allergy Appl. Immunol. 73: 155161.

94. Barfoot, R., S. Denham, L.A. Gyure, J.G. Hall, S.M. Hobbs, L.E. Jackson, and D. Robertson. 1989. Some properties of dendritic macrophages from peripheral lymph. Immunol. 68: 233-239.

95. Harkiss, G.D., J. Hopkins, and I. McConnell. 1990. Uptake of antigen by afferent lymph dendritic cells mediated by antibody. Eur. J. Immunol. 20: 2367-2373.

96. Bujdoso, R., J. Hopkins, B.M. Dutia, P. Young, and I. McConnell. 1989. Characterization of sheep afferent lymph dendritic cells and their role in antigen carriage. J. Exp. Med. 170: 1285-301.

97. Austyn, J.M. 1992. Antigen uptake and presentation by dendritic leukocytes. Semin. Immunol. 4: 227-236.

98. Silberberg-Sinakin, I., G.J. Thorbecke, R. Baer, S.A. Rosenthal, and V. Berezowsky. 1976. Antigen-bearing Langerhans cells in skin, dermal lymphatics and in lymph nodes. Cell. Immunol. 25: 137-151.

99. Knight, S.C., J. Krejci, M. Malkovsky, V. Colizzi, A. Gautam, and G.L. Asherson. 1985. The role of dendritic cells in the initiation of immune responses to contact sensitizers. I. In vivo exposure to antigen. Cell. Immunol. 94: 427-434.

100. Macatonia, S.E., A.J. Edwards, and S.C. Knight. 1986. Dendritic cells and the initiation of contact sensitivity to fluorescein isothiocyanate. Immunol. 59: 509-14.

101. Macatonia, S.E., S.C. Knight, A.J. Edwards, S. Griffiths, and P. Fryer. 1987. Localization of antigen on lymph node dendritic cells after exposure to the contact sensitizer fluorescein isothiocyanate. Functional and morphological studies. J. Exp. Med. 166: 1654-67.

102. Cumberbatch, M. and I. Kimber. 1990. Phenotypic characteristics of antigenbearing cells in the draining lymph nodes of contact sensitized mice. Immunol. 71: $404-410$.

103. Kripke, M.L., C.G. Munn, A. Jeevan, J.M. Tang, and C. Bucana. 1990. Evidence that cutaneous antigen-presenting cells migrate to regional lymph nodes during contact sensitization. J. Immunol. 145: 2833-2838.

104. Larsen, C., R. Steinman, M. Witmer-Pack, D. Hankins, P. Morris, and J. Austyn. 1990. Migration and maturation of Langerhans cells in skin transplants and explants. J. Exp. Med 172: 1483-1493.

105. Cruz Jr., P.D., R.E. Tigelaar, and P.R. Bergstresser. 1990. Langerhans cells that migrate to skin after intravenous infusion regulate the induction of contact hypersensitivity. J. Immunol. 144: 2486-2492.

106. Kaplan, G., A. Nusrat, M. Witmer, I. Nath, and Z.A. Cohn. 1987. Distribution and 
turnover of Langerhans cells during delayed immune reponses in human skin. $J$. Exp. Med. 165: 763-776.

107. Kupiec-Weglinski, J.W., J.M. Austyn, and P.J. Morris. 1988. Migration patterns of dendritic cells in the mouse. Traffic from the blood, and T cell-dependent andindependent entry to lymphoid tissues. J. Exp. Med. 167: 632-45.

108. Oluwole, S.F., K. Engelstad, R.C. De, T.S. Wang, R.A. Fawwaz, K. Reemtsma, and M.A. Hardy. 1991. Migration patterns of dendritic cells in the rat: comparison of the effects of gamma and UV-B irradiation on the migration of dendritic cells and lymphocytes. Cell. Immunol. 133: 390-407.

109. Larsen, C.P., P.J. Morris, and J.M. Austyn. 1990. Migration of dendritic leukocytes from cardiac allografts into host spleens. A novel pathway for initiation of rejection. J. Exp. Med. 171: 307-314.

110. Spencer, S.C. and J.W. Fabre. 1990. Characterization of the tissue macrophage and the interstitial dendritic cell as distinct leukocytes normally resident in the connective tissue of rat heart. J. Exp. Med. 171: 1841-1851.

111. Shah, P.D., J. Keij, S.M. Gilbertson, and D.A. Rowley. 1986. Thy-1 ${ }^{+}$and Thy-1natural killer cells. Only Thy-1- natural killer cells suppress dendritic cells. J. Exp. Med. 163: 1012-1017.

112. MacPherson, G.G. 1989. Lymphoid dendritic cells: their life history and roles in immune responses. Res. Immunol. 140: 877-926.

113. Bowers, W.E. and M.R. Berkowitz. 1986. Differentiation of dendritic cells in cultures of rat bone marrow cells. J. Exp. Med. 163: 872-883.

114. Reid, C.D., P.R. Fryer, C. Clifford, A. Kirk, J. Tikerpae, and S.C. Knight. 1990. Identification of hematopoietic progenitors of macrophages and dendritic Langerhans cells (DL-CFU) in human bone marrow and peripheral blood. Blood 76: $1139-49$.

115. Steinman, R.M., D.S. Lustig, and Z.A. Cohn. 1974. Identification of a novel cell type in peripheral lymphoid organs of mice: III. Functional properties in vivo. $J$. Exp. Med. 139: 1431-1445.

116. Austyn, J.M. and C.P. Larsen. 1990. Migration patterns of dendritic leukocytes: implications for transplantation. Transplantation 48: 1-7.

117. Nussenzweig, M.C., R.M. Steinman, J.C. Unkeless, M.D. Witmer, B. Gutchinov, and Z.A. Cohn. 1981. Studies of the cell surface of mouse dendritic cells and other leukocytes. J. Exp. Med. 154: 168-187.

118. Agger, R., M. Witmer-Pack, N. Romani, H. Stossel, W.J. Swiggard, J.P. Metlay, E. Storozynsky, P. Freimuth, and R.M. Steinman. 1992. Two populations of splenic dendritic cells detected with M342, a new monoclonal to an intracellular antigen of interdigitating dendritic cells and some B lymphocytes. J. Leuk. Biol. 52: $34-42$. 
119. Ravetch, J.V. and J.-P. Kinet. 1991. Fc receptors. Annu. Rev. Immunol. 9: 457-492.

120. Schuler, G. and R.M. Steinman. 1985. Murine epidermal Langerhans cells mature into potent immunostimulatory dendritic cells in vitro. J. Exp. Med. 161: 526-546.

121. Kämpgen, E., N. Koch, F. Koch, P. Stöger, C. Heufler, G. Schuler, and N. Romani. 1991. Class II major histocompatibility complex molecules of murine dendritic cells: synthesis, sialylation of invariant chain, and antigen processing capacity are down-regulated upon culture. Proc. Natl. Acad. Sci. USA 88: 3014-3018.

122. Larsen, C.P., S.C. Ritchie, T.C. Pearson, P.S. Linsley, and R.P. Lowry. 1992. Functional expression of the costimulatory molecules, B7/BB1, on murine dendritic cell populations. J. Exp. Med. 176: 1215-1220.

123. Romani, N., A. Lenz, H. Glassel, H. Stossel, U. Stanzl, O. Majdic, P. Fritsch, and G. Schuler. 1989. Cultured human Langerhans cells resemble lymphoid dendritic cells in phenotype and function. J. Invest. Dermatol. 93: 600-609.

124. Teunissen, M.B.M., J. Wormmeester, S.R. Krieg, P.J. Peters, I.M.C. Vogels, M.L. Kapsenberg, and J.D. Bos. 1990. Human epidermal Langerhans cells undergo profound morphologic and phenotypical changes during in vitro culture. J. Invest. Dermatol. 94: 166-173.

125. Romani, N., M. Witmer-Pack, M. Crowley, S. Koide, G. Schuler, K. Inaba, and R.M. Steinman. 1991. Langerhans cells as immature Langerhans cells. In Epidermal Langerhans cells. G. Schuler, editor. CRC Press: Boca Raton. 191-216.

126. Crowley, M.T., K. Inaba, P.M. Witmer, S. Gezelter, and R.M. Steinman. 1990. Use of the fluorescence activated cell sorter to enrich dendritic cells from mouse spleen. J. Immunol. Methods 133: 55-66.

127. Vremec, D., M. Zorbas, R. Scollay, D.J. Saunders, C.F. Ardavin, L. Wu, and K. Shortman. 1992. The surface phenotype of dendritic cells purified from mouse thymus and spleen: investigation of the CD8 expression by a subpopulation of dendritic cells. J. Exp. Med. 176: 47-58.

128. Inaba, K., G. Schuler, M.D. Witmer, J. Valinsky, B. Atassi, and R. Steinman. 1986. Immunologic properties of purified epidermal Langerhans cells: Distinct requirements for stimulation of unprimed and sensitized T lymphocytes. J. Exp. Med. 164: 605-613.

129. Inaba, K. and R.M. Steinman. 1986. Accessory cell-T lymphocyte interactions. Antigen-dependent and-independent clustering. J. Exp. Med. 163: 247-261.

130. Romani, N., S. Koide, M. Crowley, P.M. Witmer, A.M. Livingstone, C.G. Fathman, K. Inaba, and R.M. Steinman. 1989. Presentation of exogenous protein antigens by dendritic cells to $\mathrm{T}$ cell clones. Intact protein is presented best by immature, epidermal Langerhans cells. J. Exp. Med. 169: 1169-78.

131. Aiba, S. and S.I. Katz. 1991. The ability of cultured Langerhans cells to process 
and present protein antigens is MHC-dependent. J. Immunol. 146: 2479-2487.

132. Shimada, S., S.W. Caughman, S.O. Sharrow, D. Stephany, and S.I. Katz. 1987. Enhanced antigen-presenting capacity of cultured Langerhans' cells is associated with markedly increased expression of Ia antigen. J. Immunol. 139: 2551-2555.

133. Stössel, H., F. Koch, E. Kämpgen, P. Stöger, A. Lenz, C. Heufler, N. Romani, and G. Schuler. 1990. Disappearance of certain acidic organelles (endosomes and Langerhans cell granules) accompanies loss of antigen processing capacity upon culture of epidermal Langerhans cells. J. Exp. Med. 172: 1471-1482.

134. Girolomoni, G., D.K. Stone, P.R. Bergstresser, and P.J. Cruz. 1991. Vacuolar acidification and bafilomycin-sensitive proton translocating ATPase in human epidermal Langerhans cells. J. Invest. Dermatol. 96: 735-41.

135. Girolomoni, G., P.D. Cruz Jr., and P.R. Bergstresser. 1990. Internalization and acidification of surface HLA-DR molecules by epidermal Langerhans cells: a paradigm for antigen processing. J. Invest. Dermatol. 94: 753-760.

136. Witmer-Pack, M.D., J. Valinsky, W. Olivier, and R.M. Steinman. 1988. Quantitation of surface antigens on cultured murine epidermal Langerhans cells: rapid and selective increase in the level of surface MHC products. J. Invest. Dermatol. 90: 387-394.

137. Witmer-Pack, M.D., W. Olivier, J. Valinsky, G. Schuler, and R.M. Steinman. 1987. Granulocyte/macrophage colony-stimulating factor is essential for the viability and function of cultured murine epidermal Langerhans cells. J. Exp. Med. 166: 1484-1498.

138. Heufler, C., F. Koch, and G. Schuler. 1988. Granulocyte-macrophage colonystimulating factor and interleukin-1 mediate the maturation of murine epidermal Langerhans cells into potent immunostimulatory dendritic cells. J. Exp. Med. 167: 700-705.

139. Koch, F., C. Heufler, E. Kämpgen, D. Schneeweiss, G. Böck, and G. Schuler. 1990. Tumor necrosis factor alpha maintains the viability of murine epidermal Langerhans cells in culture but in contrast to granulocyte/macrophage colonystimulating factor without inducing their functional maturation. J. Exp. Med. 171: $159-171$.

140. Koch, F., E. Kämpgen, G. Schuler, and N. Romani. 1992. Effective enrichment of murine epidermal Langerhans cells by a modified- "mismatched"- panning technique. J. Invest. Dermatol. in press.

141. Kupper, T.S. 1990. Immune and inflammatory processes in cutaneous tissues: mechanisms and speculations. J. Clin. Invest. 86: 1783-1789.

142. Ishii, T., L.J. Walsh, G.J. Seymour, and R.N. Powell. 1990. Modulation of Langerhans cell surface antigen expression by recombinant cytokines. J. Oral Pathol. Med. 19: 355-9. 
143. Girolomoni, G., J.C. Simon, P.R. Bergstresser, and P.J. Cruz. 1990. Freshly isolated spleen dendritic cells and epidermal Langerhans cells undergo similar phenotypic and functional changes during short-term culture. J. Immunol. 145: $2820-6$.

144. De Bruijn, M.L.H., J.D. Nieland, C.V. Harding, and C.J.M. Melief. 1992. Processing and presentation of intact hen egg-white lysozyme by dendritic cells. Eur. J. Immunol. 22: 2347-2352.

145. Inaba, K., N. Romani, and R.M. Steinman. 1989. An antigen-independent contact mechanism as an early step in T cell proliferative responses to dendritic cells. $J$. Exp. Med. 170: 527-542.

146. Austyn, J.M., D.E. Weinstein, and R.M. Steinman. 1988. Clustering with dendritic cells precedes and is essential for T-cell proliferation in a mitogenesis model. Immunol. 63: 691-6.

147. Austyn, J.M. and P.J. Morris. 1988. T-cell activation by dendritic cells: CD18dependent clustering is not sufficient for mitogenesis. Immunol. 63: 537-43.

148. Metlay, J.P., E. Puré, and R.M. Steinman. 1989. Control of the immune response at the level of antigen-presenting cells: a comparison of the function of dendritic cells and B lymphocytes. Adv. Immunol. 47: 45-116.

149. Simon, J.C., P.J. Cruz, R.E. Tigelaar, R.D. Sontheimer, and P.R. Bergstresser. 1991. Adhesion molecules CD11a, CD18, and ICAM-1 on human epidermal Langerhans cells serve a functional role in the activation of alloreactive T cells. $J$. Invest. Dermatol. 96: 148-51.

150. King, P.D. and D.R. Katz. 1990. Mechanisms of dendritic cell function. Immunol Today 11: 206-11.

151. Boog, C.J., J.J. Neefjes, J. Boes, H.L. Ploegh, and C.J. Melief. 1989. Specific immune responses restored by alteration in carbohydrate chains of surface molecules on antigen-presenting cells. Eur. J. Immunol. 19: 537-42.

152. June, C.H., J.A. Ledbetter, P.S. Linsley, and C.B. Thompson. 1990. Role of the CD28 receptor in T-cell activation. Immunol. Today 11: 211-216.

153. Fossum, S. 1980. The architecture of rat lymph nodes. IV. Distribution of ferritin and colloidal carbon in the draining lymph nodes after footpad injection. Scand. J. Immunol. 12: 433-441.

154. Kaye, P.M., B.M. Chain, and M. Feldmann. 1985. Non-phagocytic dendritic cells are effective accessory cells for anti-mycobacterial responses in vitro. J. Immunol. 134: $1930-1934$.

155. Kapsenberg, M.L., M.B.M. Teunissen, F.E.M. Stiekema, and H.G. Keizer. 1986. Antigen-presenting cell function of dendritic cells and macrophages in proliferative $\mathrm{T}$ cell responses to soluble and particulate antigens. Eur. J. Immunol. 16: 345-350. 
156. Chain, B., P. Kay, and M. Feldman. 1986. The cellular pathway of antigen presentation: biochemical and functional analysis of antigen processing in dendritic cells and macrophages. Immunol. 58: 271-276.

157. Nair, S., F. Zhou, R. Reddy, L. Huang, and B.T. Rouse. 1992. Soluble proteins delivered to dendritic cells via $\mathrm{pH}$-sensitive liposomes induce primary cytotoxic $\mathrm{T}$ lymphocyte responses in vitro. J. Exp. Med. 175: 609-612.

158. Moore, M.W., F.R. Carbone, and M.J. Bevan. 1988. Introduction of soluble protein into the class I pathway of antigen processing and presentation. Cell 54: 777- 785.

159. Sornasse, T., V. Flamand, G. De Becker, H. Bazin, F. Tielemans, K. Thielemans, J. Urbain, L. Oberdan, and M. Moser. 1992. Antigen-pulsed dendritic cells can efficiently induce an antibody response in vivo. J. Exp. Med. 175: 15-21.

160. Liu, L.M. and G.G. MacPherson. 1991. Lymph-borne (veiled) dendritic cells can acquire and present intestinally administered antigens. Immunol. 73: 281-286.

161. Shelley, W.B. and L. Juhlin. 1976. Langerhans cells form a reticuloendothelial trap for external contact antigen. Nature 261: 46-47.

162. Søeberg, B., T. Sumerska, and B.M. Balfour. 1976. The role of the afferent lymph in the induction of contact sensitivty. Adv. Exp. Med. Biol. 66: 191-196.

163. Søeberg, B., T. Sumererska, R.M. Binns, and B.M. Balfour. 1978. Contact sensitivity in the pig. Int. Arch. Allergy Appl. Immunol. 57: 114-125.

164. Wolff, K. and E. Schreiner. 1970. Uptake, intracellular transport and degradation of exogenous protein by Langerhans cells. J. Invest. Dermatol. 54: 37-47.

165. Parr, M.B., L. Kepple, and E.L. Parr. 1991. Antigen recognition in the female reproductive tract. II. Endocytosis of horseradish peroxidase by Langerhans cells in murine vaginal epithelium. Biol. Reprod. 45: 261-5.

166. Barbey, S., F. Jaubert, M. Grun, and C. Nezelof. 1981. Skin Langerhans cells fail to trap bacterial antigen in non-sensitized guinea-pig. Ann. Immunol. (Paris) 191: 111-124.

167. Saloga, J., J. Knop, and G. Kolde. 1988. Ultrastructural cytochemical visualization of chromium in the skin of sensitized guinea pigs. Arch. Dermatol. Res. 280: 214 9.

168. Silberberg-Sinakin, I., M. Fedorko, R.L. Baer, S.A. Rosenthal, V. Berezowsky, and G.J. Thorbecke. 1977. Langerhans cells: target cells in immune complex reactions. Cell. Immunol. 32: 400-416.

169. Bieber, T., H. De La Salle, A. Wollenberg, J. Hakimi, R. Chizzonite, J. Ring, D. Hanau, and C. De La Salle. 1992. Human epidermal Langerhans cells express the high affinity receptor for immunoglobulin E (FcERI). J. Exp. Med. 175: 1285-1290.

170. Bieber, T., A. Rieger, C. Neuchrist, J.C. Prinz, E.P. Rieber, N.G. Boltz, O. 
Scheiner, D. Kraft, J. Ring, and G. Stingl. 1989. Induction of FcعR2/CD23 on human epidermal Langerhans cells by human recombinant interleukin 4 and gamma interferon. J. Exp. Med. 170: 309-14.

171. Mudde, G.C., F.C. Van Reijsen, G.J. Boland, G.C. De Gast, P.L.B. Bruynzeel, and C.A.F.M. Bruynzeel-Koomen. 1990. Allergen presentation by epidermal Langerhans' cells from patients with atopic dermatitis is mediated by IgE. Immunol. 69: $335-341$.

172. Ishii, M., Y. Terao, J. Kitajima, and T. Hamada. 1984. Sequential production of Birbeck granules through adsorptive pinocytosis. J. Invest. Dermatol. 82: 28-9.

173. Takigawa, M., K. Iwatsuki, M. Yamada, H. Okamoto, and S. Imamura. 1985. The Langerhans cell granule is an adsorptive endocytic organelle. J. Invest. Dermatol. 85: $12-5$.

174. Hanau, D., M. Fabre, D.A. Schmitt, J.L. Stampf, J.C. Garaud, T. Bieber, E. Grosshans, C. Benezra, and J.P. Cazenave. 1987. Human epidermal Langerhans cells internalize by receptor-mediated endocytosis T6 (CD1 "NA1/34") surface antigen. Birbeck granules are involved in the intracellular traffic of the T6 antigen. J. Invest. Dermatol. 89: 172-7.

175. Hanau, D., M. Fabre, D.A. Schmitt, J.C. Garaud, G. Pauly, M.M. Tongio, S. Mayer, and J.P. Cazenave. 1987. Human epidermal Langerhans cells cointernalize by receptor-mediated endocytosis "nonclassical" major histocompatibility complex class I molecules (T6 antigens) and class II molecules (HLA-DR antigens). Proc. Natl. Acad. Sci. U.S.A. 84: 2901-5.

176. Ray, A., D. Schmitt, D.C. Dezutter, M.C. Fargier, and J. Thivolet. 1989. Reappearance of CD1a antigenic sites after endocytosis on human Langerhans cells evidenced by immunogoldrelabeling. J. Invest. Dermatol. 92: 217-24.

177. Xia, W.J., E.E. Schneeberger, K. McCarthy, and R.L. Kradin. 1991. Accessory cells of the lung. II. $\mathrm{Ia}^{+}$pulmonary dendritic cells display cell surface antigen heterogeneity. Am. J. Respir. Cell. Mol. Biol. 5: 276-83.

178. Bhardwaj, N., S.M. Friedman, B.C. Cole, and A.J. Nisanian. 1992. Dendritic cells are potent antigen-presenting cells for microbial superantigens. J. Exp. Med. 175: 267-273.

179. Pancholi, P., R.M. Steinman, and N. Bhardwaj. 1992. Dendritic cells efficiently immunoselect mycobacterial-reactive $\mathrm{T}$ cells in human blood, including clonable antigen-reactive precursors. Immunol. 76: 217-224.

180. Guidos, C., A.A. Sinha, and K.C. Lee. 1987. Functional differences and complementation between dendritic cells and macrophages in $\mathrm{T}$ cell activation. Immunol. 61: 269-276.

181. Katz, D.R., M. Feldmann, R. Tees, and M.H. Schreier. 1986. Heterogeneity of accessory cells interacting with T-helper clones. Immunol. 58: 167-172. 
182. Mayrhofer, G.. P.G. Holt, and J.M. Papadimitriou. 1986. Functional characteristics of the veiled cells in afferent lymph from rat intestine. Immunol. 58: 379-387.

183. Poulter, L.W., L.A. Collings, K.S. Tung, and M.F.R. Waters. 1984. Parasitism of antigen presenting cells in hyperbacillary leprosy. Clin. Exp. Immunol. 55: 611617.

184. Mittag, H. and G. Klingmuller. 1983. Langerhans cells in granulomatous syphilis. Arch. Dermatol. Res. 275: 190-6.

185. Schuler, G., N. Romani, H. Stössel, and K. Wolff. 1991. Structural organization and biological properties of Langerhans cells. In Epidermal Langerhans cells. G. Schuler, editor. CRC Press: Boca Raton. 87-135.

186. Katz, D.R. and G.H. Sunshine. 1986. Comparative accessory cell function of Langerhans cells isolated from mouse skin. J. Exp. Pathol. 67: 157-166.

187. Parr, M.B., L. Kepple, and E.L. Parr. 1991. Langerhans cells phagocytose vaginal epithelial cells undergoing apoptosis during the murine estrous cycle. Biol. Reprod. 45: $252-60$.

188. Fossum, S. and B. Rolstad. 1986. The roles of interdigitating cells and natural killer cells in the rapid rejection of allogeneic lymphocytes. Eur. J. Immunol. 16: 440 450 .

189. Rosen, H. and S. Gordon. 1987. Monoclonal antibody to the murine type 3 complement receptor inhibits adhesion of myelomonocytic cells in vitro and inflammatory cell recruitment in vivo. J. Exp. Med. 166: 1685-1701.

190. Rosen, H. 1990. Role of CR3 in induced myelomonocytic recruitment: insights from in vivo monoclonal antibody studies in the mouse. J. Leuk. Biol. 48: 465-469.

191. Silverstein, S.C., S. Greenberg, F. Di Virgilio, and T.H. Steinberg. 1989. Phagocytosis. In Fundamental Immunology. W.E. Paul, editor. Raven Press Ltd: New York. 703-720.

192. Newman, S.L., L.K. Mikus, and M.A. Tucci. 1991. Differential requirements for cellular cytoskeleton in human macrophage complement receptor- and Fc receptormediated phagocytosis. J. Immunol. 146: 967-974.

193. Wright, S.D. and S.C. Silverstein. 1986. Overview: the function of receptors in phagocytosis. In Handbook of Experimental Immunology. 2. Cellular immunology. D.M. Weir, L.A. Herzenberg, C. Blackwell, and L.A. Herenberg, editors. Blackwell Scientific Publications: Oxford. 41.1-41.14.

194. Griffin Jr., F.M., J.A. Griffin, J.E. Leider, and S.C. Silverstein. 1975. Studies on the mechanism of phagocytosis. I. Requirements for cicumferential attachment of particle-bound ligands to specific receptors on the macrophage plasma membrane. J. Exp. Med. 142: 1263-1282. 
195. Griffin Jr., F.M., J.A. Griffin, and S.C. Silverstein. 1976. Studies on the mechanism of phagocytosis. II. The interaction of macrophages with anti-immunoglobulin IgG-coated bone marrow-derived lymphocytes. J. Exp. Med. 144: 788-809.

196. Rabinovitch, M. 1967. The dissociation of the attachment and ingestion phases of phagocytosis by macrophages. Exp. Cell Res. 46: 19-28.

197. Muller, W.A., R.M. Steinman, and Z.A. Cohn. 1983. Membrane proteins of the vacuolar system. III. Further studies on the composition and recycling of endocytic vacuole membrane in cultured macrophages. J. Cell Biol. 96: 29-36.

198. Mellman, I.S., H. Plutner, R.M. Steinman, J.C. Unkeless, and Z.A. Cohn. 1983. Internalization and degradation of macrophage $\mathrm{Fc}$ receptors during receptormediated phagocytosis. J. Cell Biol. 96: 887-895.

199. Mayorga, L.S., F. Bertini, and P.D. Stahl. 1991. Fusion of newly formed phagosomes with endosomes in intact cells and in a cell-free system. J. Biol. Chem. 266: 6511-6517.

200. Pitt, A., L.S. Mayorga, A.L. Schwartz, and P.D. Stahl. 1992. Transport of phagosomal components to an endosomal compartment. J. Biol. Chem. 267: 126132.

201. Veras, P.S.T., C. de Chastellier, and M. Rabinovitch. 1992. Transfer of zymosan (yeast cell walls) to the parasitophorous vacuoles of macrophages infected with Leishmania amazonensis. J. Exp. Med. 176: 639-646.

202. Ezekowitz, R., K. Sastry, P. Bailly, and A. Warner. 1990. Molecular characterization of the human macrophage mannose receptor: demonstration of multiple carbohydrate recognition-like domains and phagocytosis of yeasts in Cos1 cells. J. Exp. Med. 172: 1785-1794.

203. Ezekowitz, R.A.B., D.J. Williams, H. Koziel, M.Y.K. Armstrong, A. Warner, F.F. Richards, and R.M. Rose. 1991. Uptake of Pneumocystis carinii mediated by macrophage mannose receptor. Nature 351: 155-158.

204. Wolff, K. and K. Konrad. 1972. Phagocytosis of latex beads by epidermal keratinocytes in vivo. J. Ultrastruct. Res. 39: 262-280.

205. Weinshank, R.L., A.D. Luster, and J.V. Ravetch. 1988. Function and regulation of a murine macrophage-specific IgG Fc receptor, Fc $\gamma \mathrm{R}-\alpha . J$. Exp. Med. 167: 19091925.

206. Suzuki, T. 1991. Signal transduction mechanisms through Fc $\gamma$ receptors on the mouse macrophage surface. FASEB J. 5: 187-193.

207. Joiner, K.A., S.A. Fuhrman, H.M. Miettinen, L.H. Kasper, and I. Mellman. 1990. Toxoplasma gondii: fusion competence of parasitophorous vacuoles in Fcreceptor-transfected fibroblasts. Science 249: 641-646. 
208. Ross, G.D., M.J. Walport, and N. Hogg. 1989. Receptors for IgG Fc and fixed C3. In Human monocytes. M. Zembala and G.L. Asherson, editors. Academic Press: London. 123-139.

209. Wright, S.D. and S.C. Silverstein. 1982. Tumor-promoting phorbol esters stimulate $\mathrm{C} 3 \mathrm{~b}$ and $\mathrm{C} 3 \mathrm{~b}^{\prime}$ receptor-mediated phagocytosis in cultured human monocytes. $J$. Exp. Med. 156: 1149-1164.

210. Wright, S.D., L.S. Craigmyle, and S.C. Silverstein. 1983. Fibronectin and serum amyloid $\mathrm{P}$ component stimulate $\mathrm{C} 3 \mathrm{~b}-$ and $\mathrm{C} 3 \mathrm{bi}-$ mediated phagocytosis in cultured human monocytes. J. Exp. Med. 158: 1338-1343.

211. Wright, S.D., M.R. Licht, L.S. Craigmyle, and S.C. Silverstein. 1984. Communication between receptors for different ligands on a single cell: ligation of fibronectin receptors induces a reversible alteration in the function of complement receptors on cultured human monocytes. J. Cell Biol. 99: 336-339.

212. Wright, S.D. and S.C. Silverstein. 1983. Receptors for C3b and C3bi promote phagocytosis but not release of toxic oxygen from human phagocytes. J. Exp. Med. 158: 2016-2023.

213. Russell, D.G. and S.D. Wright. 1988. Complement receptor type 3 (CR3) binds to an Arg-Gly-Asp-containing region of the major surface glycoprotein, gp63, of Leishmania promastigotes. J. Exp. Med. 168: 279-292.

214. Relman, D., E. Tuomanen, S. Falkow, D.T. Golenbock, K. Saukkonen, and S.D. Wright. 1990. Recognition of a bacterial adhesin by an integrin: macrophage CR3 $\left(\alpha_{M} \beta_{2}, C D 11 b / C D 18\right)$ binds filamentous hemagglutinin of Bordetella pertussis. Cell 61: 1375-1382.

215. Brown, E.J. 1991. Complement receptors and phagocytosis. Curr. Opin. Immunol. 3: 76-82.

216. Graham, I.L., H.D. Gresham, and E.J. Brown. 1989. An immobile subset of plasma membrane $\mathrm{CD} 1 \mathrm{~b} / \mathrm{CD} 18$ (Mac-1) is involved in phagocytosis of targets recgnised by multiple receptors. J. Immunol. 142: 2352-2358.

217. Stewart, J., E.J. Glass, D.M. Weir, and M.R. Daha. 1986. Macrophage membrane receptors. In Handbook of Experimental Immunology. 2. Cellular immunology. D.M. Weir, L.A. Herzenberg, C. Blackwell, and L.A. Herenberg, editors. Blackwell Scientific Publications: Oxford. 48.1-48.17.

218. Diment, S., M.S. Leech, and P.D. Stahl. 1987. Generation of macrophage variants with 5-azacytidine: selection for mannose receptor expression. J. Leuk. Biol. 42: 485-90.

219. Halldén, G., U. Andersson, J. Hed, and S.G.O. Johansson. 1989. A new membrane permeabilization method for the detection of intracellular antigens by flow cytometry. J. Immunol. Methods 124: 103-109. 
220. Tigalonowa, M., J.R. Bjerke, J.K. Livden, and R. Matre. 1990. The distribution of

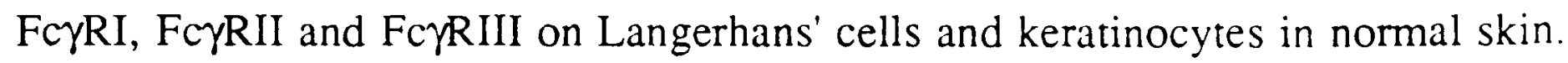
Acta Derm. Venereol. Stockh. 70: 385-90.

221. Steinman, R.M., N. Nogueira, M.D. Witmer, J.D. Tydings, and I.S. Mellman. 1980. Lymphokine enhances the expression and synthesis of Ia antigens on cultured mouse peritoneal macrophages. J. Exp. Med. 152: 1248-1261.

222. Lydyard, P.M. 1990. Flow cytometry developments, uses of monoclonal antibodies and cell purification. Curr. Opin. Immunol. 2: 923-926.

223. Ezekowitz, R.A., R.B. Sim, M. Hill, and S. Gordon. 1983. Local opsonization by secreted macrophage complement components. Role of receptors for complement in uptake of zymosan. J. Exp. Med. 159: 244-260.

224. Ezekowitz, R.A.B., R.B. Sim, G.G. MacPherson, and S. Gordon. 1985. Interaction of human monocytes, macrophages and polymorphonuclear leukocytes with zymosan in vitro. Role of type 3 complement receptors and macrophage-derived complement. J. Clin. Invest. 76: 2368-2376.

225. Knoll, L.J., M.A. Levy, P.D. Stahl, and J.I. Gordon. 1992. Analysis of the compartmentalization of myristoyl-CoA:Protein $N$-Myristoyltransferase in Saccharomyces cerevisiae. J. Biol. Chem. 267: 5366-5373.

226. Slot, J.W. and H.J. Geuze. 1985. A new method of preparing gold probes for multiple labeling cytochemistry. Eur. J. Cell Biol. 38: 87-93.

227. Harlow, E. and D. Lane. 1988. Antibodies: a laboratory manual. Cold Spring Harbor: Cold Spring Harbor Laboratory.

228. Di Carlo, F.J. and J.V. Fiore. 1958. On the composition of zymosan. Science 127: 756-757.

229. Michl, J., D.J. Ohlbaum, and S.C. Silversteins. 1976. 2-deoxyglucose selectively inhibits $\mathrm{Fc}$ and complement receptor-mediated phagocytosis in mouse peritoneal macrophages. J. Exp. Med. 144: 1465-1483.

230. Sung, S.J., R.S. Nelson, and S.M. Silverstein. 1983. Yeast mannans inhibit binding and phagocytosis of zymosan by mouse peritoneal macrophages. J. Cell Biol. 96: $160-166$.

231. Will, A., C. Blank, M. Röllinghoff, and H. Moll. 1992. Murine epidermal Langerhans cells are potent stimulators of an antigen-specific $T$ cell response to Leishmania major, the cause of cutaneous leishmaniasis. Eur. J. Immunol. 22: 1341-1347.

232. Pillemer, L., L. Blum, I.H. Lepow, L. Wurz, and E.W. Todd. 1956. The properdin system and immunity. III The zymosan assay of properdin. J. Exp. Med. 103: 1-13.

233. Warr, G.A. 1980. A macrophage receptor for (mannose/glucosamine)- 
glycoproteins of potential importance in phagocytic activity. Biochem. Biophy's. Res. Com. 93: 737-745.

234. Speert, D.P. and S.C. Silverstein. 1985. Phagocytosis of unopsonized zymosan by human monocyte-derived macrophages: maturation and inhibition by mannan. $J$. Leuk. Biol. 38: 655-658.

235. Czop, J.K. and K.F. Austen. 1985. A $\beta$-glucan inhibitable receptor on human monocytes: its identity with the phagocytic receptor for particulate activators of the alternative complement pathway. J. Immunol 134: 2588-2593.

236. Kadish, J.L., C.C. Choi, and J.K. Czop. 1986. Phagocytosis of unopsonised zymosan particles by trypsin-sensitive and $\beta$-glucan inhibitable receptors on bone marrow-derived murine macrophages. Immunol. Res. 5: 129-138.

237. Goldman, R. 1988. Characteristics of the $\beta$-glucan receptor of murine macrophages. Exp. Cell Res. 174: 481-490.

238. Steadman, R., M.M. Petersen, N. Topley, D. Williams, N. Matthews, B. Spur, and J.D. Williams. 1990. Differential augmentation by recombinant human tumor necrosis factor- $\alpha$ of neutrophil responses to particulate zymosan and glucan. $J$. Immunol. 144: 2712-2718.

239. Ross, G.D., J.A. Cain, and P.L. Lachmann. 1985. Membrane complement receptor type three (CR3) has lectin-like properties analogous to bovine conglutinin and functions as a receptor for zymosan and rabbit erythrocytes as well as a receptor for iC3b. J. Immunol. 134: 3307-3315.

240. Ross, G.D., J.A. Cain, B.L. Myones, S.L. Newman, and P.J. Lachmann. 1987. Specificity of membrane complement receptor type three (CR3) for $\beta$-glucans. Complement 4: 61-74.

241. Ezekowitz, R.A. and P.D. Stahl. 1988. The structure and function of vertebrate mannose lectin-like proteins. J. Cell Sci. Suppl. 9: 121-33.

242. Stahl, P.D. 1990. The macrophage mannose receptor: current status. Am. J. Respir. Cell. Mol. Biol. 2: 317-8.

243. Gordon, S. and T. Mokoena. 1989. Receptors for mannosyl structures on mononuclear phagocytes. In Human monocytes. M. Zembala and G.L. Asherson, editors. Academic Press: London. 141-150.

244. Pontow, S.E., V. Kery, and P.D. Stahl. 1992. The mannose receptor. Int. Rev. Cytol. 137B: in press.

245. Berton, G. and S. Gordon. 1983. Modulation of macrophage mannosyl-specific receptors by cultivation on immobilized zymosan. Effects on superoxide-anion release and phagocytosis. Immunol. 49: 705-715.

246. Czop, J.K. and K.F. Austen. 1985. Properties of glycans that activate the human 
alternative complement pathway and interact with the human monocyte $\beta$-glucan receptor. J. Immunol. 135: 3388-3393.

247. Konopski, Z., L.T. Rasmussen, R. Seljelid, and T. Eskeland. 1991. Phagocytosis of $\beta-1,3-D-$ glucan-derivatized microbeads by mouse peritoneal macrophages involves three different receptors. Scand. J. Immunol. 33: 297-306.

248. Czop, J.K. and K.F. Austen. 1980. Functional discrimination by human monocytes between their $\mathrm{C} 3 \mathrm{~b}$ receptors and their recognition units for particulate activators of the alternative complement pathway. J. Immunol. 125: 124-128.

249. Czop, J.K. and J. Kay. 1991. Isolation and characterization of $\beta$-glucan receptors on human mononuclear phagocytes. J. Exp. Med. 173: 1511-1520.

250. Domer, J.E. 1989. Candida cell wall mannan: A polysaccharide with diverse immunological properties. Crit. Rev. Microbiol. 17: 33-51.

251. Blank, C., H. Fuchs, K. Rappersberger, M. Röllinghoff, and H. Moll. 1992. Parasitism of epidermal Langerhans cells in experimental cutaneous Leishmaniasis. J. Infect. Dis. in press.

252. Drickamer, K. 1988. Two distinct classes of carbohydrate-recognition domains in animal lectins. J. Biol. Chem. 263: 9557-9560.

253. Taylor, M.E., J.T. Conary, M.R. Lennartz, P.D. Stahl, and K. Drickamer. 1990. Primary structure of the mannose receptor contains multiple motifs resembling carbohydrate-recognition domains. J. Biol. Chem. 265: 12156-62.

254. Taylor, M.E., K. Bezouska, and K. Drickamer. 1992. Contribution to ligand binding by multiple carbohydrate-recognition domains in the macrophage mannose receptor. J. Biol. Chem. 267: 1719-1726.

255. Stahl, P., P.H. Schlesinger, E. Sigardson, J.S. Rodman, and Y.C. Lee. 1980. Receptor-mediated pinocytosis of mannose glycoconjugates by macrophages: Characterization and evidence for receptor recycling. Cell 19: 207-215.

256. Wileman, T., R.L. Boshans, P. Schlesinger, and P. Stahl. 1984. Monensin inhibits recycling of macrophage mannose-glycoprotein receptors and ligand delivery to lysosomes. Biochem. J. 220: 665-675.

257. Blackwell, J., R.A.B. Ezekowitz, M.B. Roberts, J.Y. Channon, R.B. Sim, and S. Gordon. 1985. Macrophage complement and lectin-like receptors bind Leishmania in the absence of serum. J. Exp. Med. 162: 324-331.

258. Swanson, J.A., B.A. Yirinec, and S.C. Silverstein. 1985. Phorbol esters and horseradish peroxidase stimulate pinocytosis and redirect the flow of pinocytosed fluid in macrophages. J. Cell Biol. 100: 851-859.

259. Stewart, W.W. 1978. Functional connections between cells as revealed by dyecoupling with a highly fluorescent naphtalimide tracer. Cell 14: 741-759. 
260. Blum, J.S., P.D. Stahl, R. Diaz, and M.L. Fiani. 1991. Purification and characterization of the D-mannose receptor from $\mathrm{J} 774$ mouse macrophage cells. Carbohydr. Res. 213: 145-153.

261. Lennartz, M.R., F.S. Cole, and P.D. Stahl. 1989. Biosynthesis and processing of the mannose receptor in human macrophages. J. Biol. Chem. 264: 2385-90.

262. Wileman, T.E., M.R. Lennartz, and P.D. Stahl. 1986. Identification of the macrophage mannose receptor as a $175-\mathrm{kDa}$ membrane protein. Proc. Natl. Acad. Sci.U.S.A. 83: 2501-5.

263. Lennartz, M.R., F.S. Cole, V.L. Shepherd, T.E. Wileman, and P.D. Stahl. 1987. Isolation and characterization of a mannose-specific endocytosis receptor from human placenta. J. Biol. Chem. 262: 9942-4.

264. Lennartz, M.R., T.E. Wileman, and P.D. Stahl. 1987. Isolation and characterization of a mannose-specific endocytosis receptor from rabbit alveolar macrophages. Biochem. J. 245: 705-11.

265. Sveum, R.J., T.M. Chused, M.M. Frank, and E.J. Brown. 1986. A quantitative fluorescent method for measurement of bacterial adherence and phagocytosis. $J$. Immunol. Methods 90: 257-264.

266. Ogle, J.D., J.G. Noel, R.M. Sramkoski, C.K. Ogle, and J.W. Alexander. 1988. Phagocytosis of opsonised fluorescent microspheres by human neutrophils. A twocolor flow cytometric method for the determination of attachment and ingestion. $J$. Immunol. Methods 115: 17-29.

267. Hed, J., G. Hallden, S.G.O. Johansson, and P. Larsson. 1987. The use of fluorescence quenching in flow cytofluorometry to measure the attachment and ingestion phases in phagocytosis in peripheral blood without prior cell seperation. J. Immunol. Methods 101: 119- 125.

268. Stingl, G., E.C. Wolff-Schreiner, W.J. Pichler, F. Gschnait, W. Knapp, and K. Wolff. 1977. Epidermal Langerhans cells bear Fc and C3 receptors. Nature 268: 245-246.

269. Schmitt, D.A., D. Hanau, T. Bieber, C. Dezutter-Dambuyant, D. Schmitt, M. Fabre, G. Pauly, and J. Cazenave. 1989. Human epidermal langerhans cells express only the 40-kilodalton Fc $\gamma$ receptor (FcRII). J. Immunol. 144: 4284 4290.

270. Midoux, P., A. Roche, and M. Monsigny. 1987. Quantitation of the binding, uptake, and degradation of fluoresceinylated neoglycoproteins by flow cytometry. Cytometry 8: 327-334.

271. Stanier, R.Y., J.L. Ingraham, M.L. Wheelis, and P.R. Painter. 1987. General Microbiology. 5th ed. London: Macmillan Education Ltd.

272. Wilson, M.E. and R.D. Pearson. 1988. Roles of CR3 and mannose receptors in the attachment and ingestion of Leishmania donovani by human mononuclear 
phagocytes. Infect. Immun. 56: 363-369.

273. Roos, D., A.A.M. Bot, M.L.J. Van Schaik, M. De Boer, and M.R. Daha. 1981. Interaction between human neutrophils and zymosan particles: the role of divalent cations. J. Immunol. 126: 433-440.

274. Roecklein, J.A., R.P. Swartz, and H. Yeager Jr. 1992. Nonopsonic uptake of Mycobacterium avium complex by human monocytes and alveolar macrophages. $J$. Lab. Clin. Med. 119: 772-781.

275. Stahl, P.D., J.S. Rodman, M.J. Miller, and P.H. Schlesinger. 1978. Evidence for receptor-mediated binding of glycoproteins, glycoconjugates, and lysosomal glycosidases by alveolar macrophages. Proc. Natl. Acad. Sci. U.S.A. 75: 13991403.

276. Pimpaneau, V., P. Midoux, M. Monsigny, and A.C. Roche. 1991. Characterization and isolation of an intracellular D-mannose-specific receptor from human promyelocytic HL60 cells. Carbohydr. Res. 213: 95-108.

277. Stein, M., S. Keshav, N. Harris, and S. Gordon. 1992. Interleukin 4 potently enhances murine macrophage mannose receptor activity: a marker of alternative immunologic macrophage activation. J. Exp. Med. 176: 287-292.

278. Amigorena, S., C. Bonnerot, J.R. Drake, D. Choquet, W. Hunziker, J. Guille, P. Webster, C. Sautes, I. Mellman, and W.H. Fridman. 1992. Cytoplasmic domain heterogeneity and functions of IgG Fc receptors in B lymphocytes. Science 256: $1808-1812$.

279. Kaplan, G., G. Walsh, L.S. Guido, P. Meyn, R.A. Burkhardt, R.M. Abalos, J. Barker, P.A. Frindt, T.T. Fajardo, R. Celona, and Z.A. Cohn. 1992. Novel responses of human skin to intradermal recombinant granulocyte/macrophagecolony-stimulating factor: Langerhans cell recruitment, keratinocyte growth and enhanced wound healing. J. Exp. Med. 175: 1717-1728.

280. Okawa, Y., C.R. Howard, and M.W. Steward. 1992. Production of anti-peptide antibody in mice following immunization with peptides conjugated to mannan. $J$. Immunol. Methods 149: 127-131.

281. Simmons, B.M., P.D. Stahl, and J.H. Russell. 1987. In vivo depletion of mannose receptor bearing cells from rat liver by ricin A chain: effects on clearance of $\beta$ glucuronidase. Biochem. Biophys. Res. Commun. 146: 849-54.

282. Zenilman, M.E., M. Fiani, P.D. Stahl, E.M. Brunt, and M.W. Flye. 1989. Selective depletion of Kupffer cells in mice by intact ricin. Transplantation 47: 200-3.

283. Simmons, B.M., P.D. Stahl, and J.H. Russell. 1986. Mannose receptor-mediated uptake of ricin toxin and ricin A chain by macrophages. Multiple intracellular pathways for a chain translocation. J. Biol. Chem. 261: 7912-20. 
